\title{
THE CONSTRUCTION OF THE L3 EXPERIMENT
}

B. ADEVA ${ }^{14)}$, M. AGUILAR-BENITEZ ${ }^{20)}$, H. AKBARI 5), J. ALCARAZ 20), A. ALOISIO 22), J. ALVAREZ-TAVIEL 20), G. ALVERSON ${ }^{8)}$, M.G. ALVIGGI ${ }^{22)}$, H. ANDERHUB ${ }^{33)}$, A.L. ANDERSON ${ }^{11}$, A.M. ANGELOV ${ }^{11.30)}$, T.H. ANGELOV ${ }^{11.30)}$ ', G.H. ANTCHEV 30), L. ANTONOV 30), D. ANTREASYAN 15), A. AREFIEV 21), I.H. ATANASOV 30), B. AUROY 11),

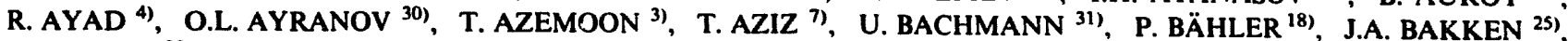

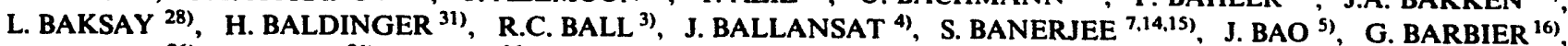
L. BARONE ${ }^{26)}$, G. BASTI ${ }^{26)}$, A. BAY ${ }^{16)}$, F. BEAUVAIS ${ }^{33)}$, U. BECKER ${ }^{11,14)}$, R. BEISSEL 1). S. BENDIG ${ }^{31)}$, P. BÉNÉ ${ }^{16)}$, J. BERDUGO ${ }^{14.20)}$, P. BERGES ${ }^{11)}$, M. BERTHET ${ }^{4)}$, Y. BERTSCH ${ }^{4)}$, B.L. BETEV ${ }^{30)}$, A. BILAND 33), A. BISCHOFF ${ }^{32)}$, M. BISCHOPS ${ }^{\text {) }}$, R. BIZZARRI ${ }^{26)}$, J.J. BLAISING ${ }^{4)}$, M. BLANC ${ }^{11, a)}$, P. BLÖMEKE ${ }^{\text {1) }}$, B. BLUMENFELD ${ }^{\text {5) }}$, G.J. BOBBINK ${ }^{2)}$, M. BOCCIOLINI ${ }^{12)}$, K.D. BOFFIN ${ }^{1)}$, W. BOHLEN ${ }^{31)}$, A. BÖHM ${ }^{1)}$, T. BÖHRINGER ${ }^{18)}$, H. BONNEFON ${ }^{4)}$, C. BOPP ${ }^{25)}$, B. BORGIA ${ }^{26)}$, K. BOSSELER "), J.F. BOTTOLIER ${ }^{4)}$, M. BOURQUIN ${ }^{16)}$, D. BOUTIGNY 4), P. BOWDITCH 11.b)'

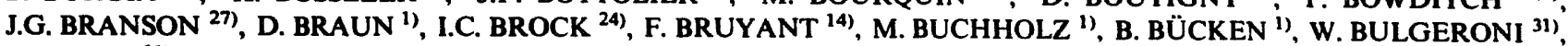
R. BUREL ${ }^{11)}$, J.D. BURGER ${ }^{11}$, C. BURGOS ${ }^{20)}$, J.P. BURQ ${ }^{19)}$, L. CAIAZZO ${ }^{22)}$, M. CAILLAT ${ }^{4)}$, B. CAMBERLIN ${ }^{4)}$, D. CAMPANA ${ }^{22)}$, C. CAMPS $^{1)}$, V. CANALE ${ }^{26)}$, M. CAPELL ${ }^{3)}$, F. CARBONARA ${ }^{22)}$, F. CARMINATI ${ }^{12)}$, A.M. CARTACCI ${ }^{12)}$, M. CERRADA ${ }^{20)}$, F. CESARONI ${ }^{26)}$, Y.H. CHANG ${ }^{11)}$, J.W. CHAPMAN ${ }^{3)}$, M. CHEMARIN ${ }^{19)}$, A. CHEN ${ }^{34)}$, C. CHEN ${ }^{6)}$, H.F. CHEN ${ }^{17)}$, H.S. CHEN ${ }^{6)}$, M. CHEN ${ }^{11)}$, M.L. CHEN ${ }^{14)}$, S.R. CHENDVANKAR ${ }^{\text {) }}$, G. CHEVENIER ${ }^{33)}$, S. CHIDZIK ${ }^{\text {5), }}$ G. CHIEFARI ${ }^{22)}$, C.Y. CHIEN ${ }^{5)}$, F. CHOLLET ${ }^{4)}$, M. CHUMAKOV ${ }^{21)}$, C. CIVININI ${ }^{12)}$, I. CLARE 11), R. CLARE ${ }^{11)}$, G. COIGNET ${ }^{4)}$, N. COLINO ${ }^{20)}$, V. COMMICHAU ${ }^{1)}$, G. CONFORTO ${ }^{12)}$, P. CRISTOFORI ${ }^{26)}$, F. CRIJNS ${ }^{2)}$, X.Y. CUI ${ }^{6,15)}$, T.S. DAI ${ }^{11)}$, R. D'ALESSANDRO ${ }^{12)}$, M. DANIEL ${ }^{20)}$, X. DE BOUARD ${ }^{4) \dagger}$, B. DEBYE ${ }^{1)}$, G. DECREUSE ${ }^{11}$, A. DEGRÉ ${ }^{4)}$, K. DEITERS ${ }^{32)}$, E. DÉNES ${ }^{9)}$, P. DENES ${ }^{25)}$, F. DeNOTARISTEFANI ${ }^{26)}$, M. DEUTSCHMANN ${ }^{1)}$, M. DHINA ${ }^{33)}$, B. DIDIERJEAN ${ }^{4)}$, M. DIEMOZ ${ }^{26)}$, M. DIETRICH ${ }^{11)}$, H.A. DIMITROV ${ }^{30)}$, C. DIONISI ${ }^{26)}$, F. DITTUS ${ }^{23)}, M^{\prime}$. DOHMEN ${ }^{1}$ ', R. DOLIN ${ }^{11}$, J.F. DONAHUE ${ }^{11}$, A. DONAT ${ }^{32)}$, E. DRAGO ${ }^{22)}$, K.H. DREGER ${ }^{1,}$, T. DRIEVER ${ }^{21}$, G. DROMBY ${ }^{4)}$, P. DUINKER ${ }^{2)}$, I. DURAN ${ }^{11,14,20)}$, M. ELKACIMI ${ }^{19)}$, H. ELMAMOUNI ${ }^{19)}$, A. ENGLER ${ }^{24)}$, F.J. EPPLING ${ }^{11)}$, F.C. ERNÉ ${ }^{2)}$, I. ERNE ${ }^{31)}$, H. ESSER ${ }^{11}$, P. EXTERMANN ${ }^{16)}$, R. FABBRETTI ${ }^{33)}$, G. FABER ${ }^{2.11)}$, S. FALCIANO ${ }^{26)}$, T. FALK ${ }^{25)}$, S.J. FAN ${ }^{29}$ ', M. FAVRE ${ }^{33)}$, J. FAY ${ }^{19)}$, S. FEHÉR ${ }^{28)}$, J. FEHLMANN ${ }^{33)}$, M. FELDMANN ${ }^{14)}$, H. FENKER ${ }^{8)}$, T. FERGUSON ${ }^{24)}$, M. FERNANDEZ ${ }^{20)}$, F. FERRONI ${ }^{26)}$, H. FESEFELDT "), J. FIELD ${ }^{16)}$, J.M. FIGAROLA 20), C.F. FIGUEROA ${ }^{20)}$, G.A. FILIPOV ${ }^{30}$ ), B. FOLIGNE ${ }^{11)}$, G. FORCONI ${ }^{16)}$, T. FOREMAN ${ }^{2)}$, V. FRANZKE "), W. FREI ${ }^{31}$, K. FREUDENREICH ${ }^{33)}$, W. FRIEBEL ${ }^{32)}$, M. FUKUSHIMA ${ }^{11)}$, G. GAILLARD ${ }^{4)}$, M. GAILLOUD ${ }^{18)}$, Yu. GALAKTIONOV ${ }^{21)}$, E. GALLO ${ }^{12)}$, S.N. GANGULI ${ }^{\text {7), D. GARELICK }}{ }^{8)}$, S.S. GAU ${ }^{34)}$, G. GAVRILOV ${ }^{13)}$, E. GENNARI ${ }^{26)}$, S. GENTILE 26). M. GETTNER ${ }^{8)}$, C. GIRARD ${ }^{4)}$, M. GLAUBMAN ${ }^{8)}$, S. GOLDFARB ${ }^{3)}$, Z.F. GONG ${ }^{171}$, E. GONZALEZ 20), A. GORDEEV ${ }^{211}$ Yu. GORODKOV ${ }^{21)}$, P. GÖTTLICHER ${ }^{1)}$, C. GOY $^{4)}$, M. GOYOT ${ }^{19}$, G. GRATTA ${ }^{26)}$, A. GRIMES ${ }^{\text {() }}$, C. GRINNELL $^{11.6)}$,

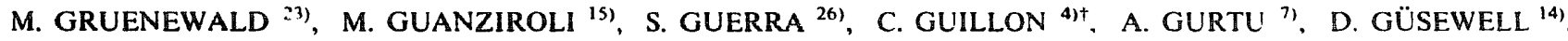
H.R. GUSTAFSON 3), M. HAENSLI 33), M. HAAN 1), C. HALLER 11), T. HAMACHER "), H. HAMMERS "), K. HANGARTER ${ }^{1}$, S. HANCKE ${ }^{1)}$, M. HARRIS ${ }^{14)}$, D. HARTING ${ }^{2)}$, F.G. HARTJES ${ }^{2)}$, C.F. HE ${ }^{29)}$, A. HEAVEY ${ }^{25}$ ), T. HEBBEKER ${ }^{14)}$, M. HEBERT ${ }^{27)}$, R. HELLER ${ }^{32}$, Ch. HELMRATH ${ }^{1)}$, J. HERRMANN ${ }^{33)}$, G. HERTEN ${ }^{11}$, U. HERTEN ${ }^{1)}$,

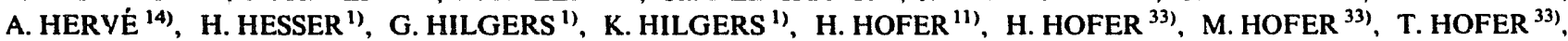
F. HOFFMANN ${ }^{1)}$, U. HORISBERGER ${ }^{33)}$, I. HORVATH ${ }^{33)}$, L.S. HSU ${ }^{34)}$, G.Q. HU ${ }^{29)}$, B. ILLE ${ }^{19)}$, M.M. ILYAS ${ }^{\text {(5) }}$, G. IMPROTA 22), V. INNOCENTE ${ }^{22)}$, E. ISIKSAL ${ }^{33)}$, E. JAGEL ${ }^{15)}$, B.N. JIN ${ }^{6)}$, L.W. JONES ${ }^{3)}$, M. JONGMANNS ${ }^{33)}$, H. JUNG ${ }^{33)}$, P. KAARET ${ }^{25)}$, O. KAELIN ${ }^{33)}$, W. KAESTLI ${ }^{33)}$, Yu. KAMYSHKOV ${ }^{21)}$, D. KAPLAN ${ }^{8}$, W. KARPINSKI ${ }^{1)}$, Y. KARYOTAKIS $^{4)}$, W. KERTZEK ${ }^{1)}$, V. KHOZE ${ }^{13)}$, G. KIRCHHOFF ${ }^{1)}$, W. KITTEL ${ }^{21}$, A. KLIMENTOV ${ }^{21)}$, P.F. KLOK ${ }^{2)}$, M. KOLLEK ${ }^{1)}$, M. KOLLER ${ }^{31)}$, A.C. KÖNIG ${ }^{2)}$, O. KORNADT ${ }^{11}$, V. KOUTSENKO ${ }^{21)}$, R.W. KRAEMER ${ }^{24)}$, V.R. KRASTEV ${ }^{30)}$, A. KRATEL ${ }^{23)}$, W. KRENZ ${ }^{1)}$, A. KUHN ${ }^{31)}$, A. KUNIN ${ }^{21)}$, S. KWAN $^{8}$, J. LACOTTE ${ }^{4)}$, M. LaMARRA $^{16)}$, G. LANDI ${ }^{12)}$, W. LANGE ${ }^{32)}$, K. LANIUS ${ }^{32)}$, D. LANSKE ${ }^{1)}$, S. LANZANO ${ }^{14.22)}$, J.M. LE GOFF ${ }^{8.14)}$, J.C. LE MAREC ${ }^{4)}$, D. LEA $^{25)}$, M. LEBEAU ${ }^{4)}$, P. LEBRUN ${ }^{19}$, P. LECOMTE ${ }^{33)}$, J. LECOQ ${ }^{4)}$, P. LECOQ ${ }^{14)}$, P. LE COULTRE ${ }^{33)}$, I. LEEDOM ${ }^{8}$, A. LEGER ${ }^{16)}$, F. LEHMANN ${ }^{1)}$, L. LEISTAM ${ }^{14)}$, R. LEISTE ${ }^{32)}$, E. LEJE('NE ${ }^{18)}$. B. LEONI ${ }^{31)}$, J. LETTRY ${ }^{33)}$, X. LEYTENS ${ }^{21}$, C. LI ${ }^{17)}$, H.T. LI ${ }^{6)}$, L. LI ${ }^{33)}$, P.J. LI ${ }^{29)}$, X.G. LI ${ }^{6)}$, J.Y. LIAO ${ }^{29)}$, Z.Y. LIN ${ }^{17)}$. F.L. LINDE ${ }^{24)}$, D. LINNHÖFER ${ }^{14)}$, E. LOFTIN ${ }^{11}$, W. LOHMANN ${ }^{32)}$, S. LÖKÖS ${ }^{28)}$, E. LONGO ${ }^{26)}$, Y.S. L. ${ }^{6)}$, I.M. LUBBERS ${ }^{2)}$, K. LÜBELSMEYER ${ }^{11}$, C. LUCI ${ }^{26)}$, D. LUCKEY ${ }^{11.15 . d)}$, X. LUE ${ }^{33)}$, L. LUMINARI ${ }^{26)}$, G. LUNADEI ${ }^{26)}$, F. LÜRKEN ${ }^{11}$, H. MA ${ }^{23)}$, W.G. MA ${ }^{17}$, M. MacDERMOTT ${ }^{33)}$, N. MADJAR ${ }^{19)}$, R. MAGAHIZ ${ }^{28)}$, M. MAIRE ${ }^{4)}$, P.K. MALHOTRA ${ }^{7)}$, A. MALININ $^{21)}$, C. MANA $^{14.20)}$, F. MANNA ${ }^{22)}$, G. MANTO ${ }^{22)}$, Y.F. MAO ${ }^{6)}$, M. MAOLINBAY ${ }^{33)}$, P. MARCHESINI 15), A. MARCHIONNI ${ }^{22)}$, M. MARKWALDER ${ }^{33)}$, P. MARSDEN ${ }^{11, e)}$, J.P. MARTIN ${ }^{14,19)}$, L. MARTINEZ 20), H.U. MARTYN ${ }^{1)}$, F. MARZANO ${ }^{26)}$, V. MARZULLO ${ }^{22)}$, F. MASCIOCCHI ${ }^{16)}$, G.G.G. MASSARO ${ }^{2)}$, L. MASSONNET ${ }^{4)}$, T. MATSUDA ${ }^{11 . n}$, G. MAURELLI ${ }^{19}$, K. MAZUMDAR ${ }^{7)}$, P. MCBRIDE ${ }^{10)}$, G. MEDICI ${ }^{26)}$, H. MEIER ${ }^{33)}$, Th. MEINHOLZ ${ }^{1}$, M. MERK ${ }^{2)}$, R. MERMOD ${ }^{16)}$, L. MEROLA 22), M. MESCHINI 12), W.J. METZGER 2), M. MICKE 1), U. MICKE 1), G.B. MILLS ${ }^{3)}$, J. MNICH "), 
M. MOELLER ", A. MOLINERO ${ }^{20}$, L. MONTANET ${ }^{14)}$, B. MONTELEONI ${ }^{12)}$, R. MONTINO ", G. MORAND ${ }^{16)}$, R. MORAND ${ }^{4)}$, S. MORGANT! ${ }^{26)}$, V. MORGUNOV ${ }^{21)}$, R. MOUNT ${ }^{23)}$, M. MOYNOT ${ }^{4)}$, P. MUGNIER ${ }^{4)}$, W. NÄGELI ${ }^{31)}$, E. NAGY ${ }^{9.14)}$, M. NAPOLITANO ${ }^{22)}$, S. NEBOUX ${ }^{4)}$, H. NEWMAN ${ }^{23)}$, Ch. NEYER ${ }^{1)}$, K. NGUYEN ${ }^{1)}$, L. NIESSEN ${ }^{16}$, A. NIKITIN ${ }^{21)}$, W.D. NOWAK ${ }^{32)}$, M. OKLE ${ }^{33)}$, P. OLMOS ${ }^{20)}$, J. ONVLEE ${ }^{2)}$, D. OSBORNE ${ }^{11}$, J. OSSMANN ${ }^{16)}$, D. PANDOULAS ${ }^{1)}$, H. PAPROTNY ${ }^{1)}$, A. PARMENTOL. ${ }^{22)}$, G. PASSEGGIO ${ }^{22)}$, G. PATERNOSTER 22), S. PATRICELLI ${ }^{22)}$, Y.J. PEI (') Y. PENG ${ }^{2)}$, Y. PENG ${ }^{32)}$, D. PERRET-GALLIX ${ }^{4)}$, J. PERRIER ${ }^{16)}$, E. PERRIN $^{16)}$, G. PERROT ${ }^{4)}$, P. PETITPAS ${ }^{4)}$,

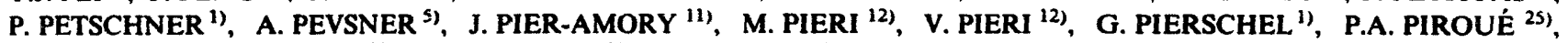
V. PLYASKIN ${ }^{21)}$, M. POHL ${ }^{33)}$, V. POJIDAEV ${ }^{21)}$, C.L.A. POLS ${ }^{2)}$, T. PONOMAREFF ${ }^{1)}$, J. POTYKA ${ }^{1)}$, N. PRODUIT ${ }^{16)}$, P. PROKOFIEV ${ }^{13)}$, F. PRUJA ${ }^{4)}$, G. PÜTZ ${ }^{1)}$, J.M. QIAN ${ }^{11}$, R. RAGHAVAN ${ }^{7}$, P. RAZIS ${ }^{33)}$, K. READ 25), P. REDDICK ${ }^{11)}$, K. REISSMANN 1), D. REN ${ }^{33)}$, S. REUCROFT ${ }^{8)}$, D. REY ${ }^{4)}$, M. REYNAUD ${ }^{19)}$, X. RICADONNA ${ }^{4)}$, J-P. RICHEUX ${ }^{16)}$, C. RIPPICH ${ }^{24)}$, U. RINSCHE 1), R. ROCCO ${ }^{22)}$, S. RODRIGUEZ ${ }^{20)}$, B.P. ROE 3), M. RÖHNER "), S. RÖHNER 1), Th. ROMBACH "), L. ROMERO ${ }^{20)}$, J. ROSE ${ }^{1)}$, U. RÖSER ${ }^{32)}$, S. ROSIER-LEES ${ }^{4,14)}$, J.A. RUBIO ${ }^{14,20)}$, W. RUCKSTUHL ${ }^{16)}$, H. RYKACZEWSKI ${ }^{11.15)}$, P. SAHUC ${ }^{19}$, J. SALICIO ${ }^{20)}$, S. SARAN ${ }^{7}$, G. SAUVAGE ${ }^{4)}$, A. SAVIN ${ }^{21)}$, T. SCHAAD ${ }^{16)}$, B. SCHAFHEITLE ${ }^{33)}$, V. SCHEGELSKY ${ }^{13)}$, A. SCHETKOVSKY ${ }^{13)}$, F. SCHILD ${ }^{31)}$, R. SCHILLSOTT ${ }^{11)}$, P. SCHMITT ${ }^{10)}$, D. SCHMITZ "), P. SCHMITZ "), M. SCHNEEGANS ${ }^{4)}$, M. SCHNEIDER ${ }^{31}$, E. SCHNEEVOGT ${ }^{1)}$, M. SCHÖNTAG "), D.J. SCHOTANUS ${ }^{2)}$, H. SCHUIJLENBURG ${ }^{33)}$, R. SCHULTE ${ }^{1)}$, A. SCHULTZ VON DRATZIG ${ }^{1)}$, K. SCHULTZE ${ }^{1}$, J. SCHWENKE "), G. SCHWERING "), C. SCIACCA ${ }^{22)}$, P.G. SEILER ${ }^{33)}$, J.C. SENS ${ }^{2)}$, I. SHEER ${ }^{27)}$, V. SHEVCHENKO ${ }^{21)}$, S. SHEVCHENKO ${ }^{21)}$, X.R. SHI ${ }^{24)}$, K. SHMAKOV ${ }^{21)}$, V. SHOUTKO ${ }^{21)}$, E. SHUMILOV ${ }^{21)}$, R. SIEDLING ${ }^{1)}$, N. SMIRNOV ${ }^{13)}$, V. SOUVOROV ${ }^{13)}$, C. SOUYRI ${ }^{4)}$, I. SPANGLER 5), T. SPICKERMANN ${ }^{1)}$, B. SPIESS ${ }^{31)}$, P. SPILLANTINI ${ }^{12)}$, R. STAROSTA "), M. STEUER ${ }^{11.15 . d), ~ D . P . ~ S T I C K L A N D ~ 25), ~ B . ~ S T O ̈ H R ~ 33), ~ H . ~ S T O N E ~ 16), ~}$

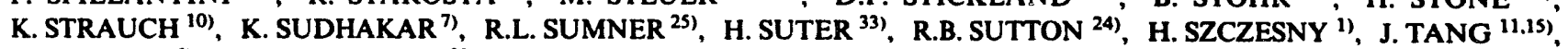
X.W. TANG ${ }^{6)}$, E. TARKOVSKY ${ }^{21)}$, A. TAVENRATH ${ }^{1)}$, V. TCHUDAKOV ${ }^{21)}$, J.M. THENARD ${ }^{4)}$, E. THOMAS ${ }^{15}$, T. THON ${ }^{32)}$, H. THUERIG ${ }^{33)}$, M. THULEN ${ }^{8)}$, C. TIMMERMANS ${ }^{2)}$, Samuel C.C. TING ${ }^{11)}$, S.M. TING ${ }^{11)}$, F. TONISCH ${ }^{32)}$, Y.P. TONG ${ }^{34)}$, M. TONUTTI "), S.C. TONWAR ${ }^{7)}$, J. TÒTH ${ }^{9)}$, W. TOTH ${ }^{11, b)}$, G. TROWITZSCH ${ }^{32)}$, K.L. TUNG ${ }^{6)}$, J. ULBRICHT ${ }^{31)}$, L. URBÀN ${ }^{9)}$, E. VALENTE ${ }^{26)}$, R.T. VAN DE WALLE ${ }^{2)}$, H. VAN DER GRAAF ${ }^{2)}$, V. VANZANELLA ${ }^{22)}$, M. VERGAIN ${ }^{11)}$, I. VETLITSKY ${ }^{21)}$, H. VEY ${ }^{4)}$, G. VIERTEL ${ }^{33)}$, M. VIVARGENT ${ }^{4)}$, H. VOGEL 24), S. VOLK.OV ${ }^{13)}$,

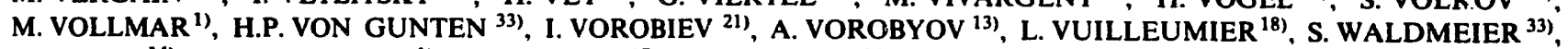
W. WALK ${ }^{14)}$, W. WALLRAFF ${ }^{1)}$, C.Y. WANG ${ }^{17}$, G.H. WANG ${ }^{24)}$, J.H. WANG ${ }^{6)}$, Q.F. WANG ${ }^{10)}$, X.L. WANG ${ }^{17}$, Y.F. WANG ${ }^{12)}$, Z.M. WANG ${ }^{11}$, Z.M. WANG ${ }^{17}$, D. WASSENBERG ${ }^{11}$, D. WEGMANN ${ }^{33)}$, R. WEILL ${ }^{18)}$, T.J. WENAUS ${ }^{11}$, P. WENGER ${ }^{31}$, J. WENNINGER ${ }^{16)}$, M. WHITE ${ }^{11}$, R. WILHELM 2), C. WILLMOTT 20), H.P. WIRTH 1), F. WITTGENSTEIN ${ }^{14)}$, R.J. WU ${ }^{6)}$, S.X. WU ${ }^{11.15)}$, Y.G. WU ${ }^{6)}$, B. WYSLOUCH ${ }^{11.14)}$, F.Y. XI ${ }^{6.11)}$, Z.Z. XU $^{17)}$, Z.L. XUE ${ }^{29}$, D.S. YAN ${ }^{29)}$, K.D. YANEV ${ }^{30)}$, B.Z. YANG ${ }^{17)}$, C.G. YANG ${ }^{6)}$, K.S. YANG ${ }^{6)}$, Q.Y.YANG ${ }^{6)}$, Z.Q. YANG ${ }^{29)}$, C.H. YE ${ }^{11.8)}$, S.C. YEH ${ }^{34)}$, Z.W. YIN ${ }^{29)}$, C. ZABOUNIDIS ${ }^{81}$, L. ZEHNDER ${ }^{33)}$, Y. ZENG ${ }^{11}$, D.H. ZHANG ${ }^{2)}$, S.Y. ZHANG ${ }^{6)}$,

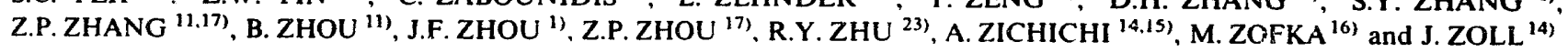

"1. Physikalisches Institut, RWTH, Aachen, FRG *;

3. Physikalisches Institut, RWTH, Aachen. FRG*

2) National Institute for High Energy Physics, NIKHEF, Amsterdam, The Netherlands NIKHEF.H and University of Nijmegen, Nijmegen. The Neiherlands

3) University of Michigan, Ann Arbor, USA

4, Laboratoire de Physique des Particules, LAPP, Annecy, France

5) Johns Hopkins University, Baltimore, USA

6) Institute of High Energy Physics, IHEP, Beijing, P.R. China

7) Tata Institute of Fundamental Research, Bombay, India

${ }^{8)}$ Northeastern University, Boston, USA

${ }^{90}$ Central Research Institute for Physics of the Hungarian Academy of Sciences, Budapest, Hungary

10) Harvard University, Cambridge, USA

11) Massachusetts Institute of Technology, Cambridge, USA

12) INFN Sezione di Firenze and University of Firenze, Italy

1.3) Leningrad Nuclear Physics Institute, Gatchina, USSR

14) European Laboratory for Particle Physics, CERN, Geneva, Snitzerland

15) World Laboratory, FBLJA Project, Geneva, Switzerland

(1). University of Geneva, Geneva, Switzeriand

17) Chinese University of Science and Technology, USTC, Hefei, P.R. China

${ }^{18)}$ University of Lausanne, Lausanne, Switzerland

19.) Institut de Physique Nucléaire de Lyon, IN2P3-CNRS/Université Claude Bernard, Villeurbanne, France

21) Center of Energy and Environmental Research, CIEMAT, Madrid, Spain * *

$\therefore$ Instituie of Theoretical and Experimental Physics, ITEP, Moscow, USSR

2.) INFN Sezione di Napoli and University of Naples, Italy'

${ }_{231}^{23}$ California institute of Technology, Pasadena, USA

24) Carnegie Mellon University, Pittsburgh, USA

25) Princeton University, Princeton, USA 
26) INFN Sezione di Roma and University of Roma, "La Sapienza", Italy

27) University of California, San Diego, USA

28) Union College, Schenectady, USA

29) Shanghai Institute of Ceramics, SIC, Shanghai, P.R. China

30) Central Laboratory of Automation and Instrumentation, CLANP, Sofia, Bulgaria

31) Paul Scherrer Institut, PSI, Würenlingen, Switzerland

32) High Energy Physics Institute, Zeuthen-Berlin, Germany

${ }^{33)}$ Eidgenössische Technische Hochschule, ETH Zürich, Switzerland

34) National Science Council, Taiwan

Received 8 November 1989

The L3 experiment is one of the six large detectors designed for the new generation of electron-positron accelerators. It is the only detector that concentrates its efforts on limited goals of measuring electrons, muons and photons. By not attempting to identify hadrons, L3 has been able to provide an order of magnitude better resolution for electrons, muons and photons. Vertices and hadron jets are also studied. The construction of $\mathrm{L} 3$ has involved much state of the art technology in new principles of vertex detection and in new crystals for large scale electromagnetic shower detection and ultraprecise muon detection. This paper presents a summary of the construction of $\mathrm{L3}$.

\section{Introduction}

The $\mathrm{L} 3$ experiment is designed to study $\mathrm{e}^{+} \mathrm{e}^{-}$collisions in the $100-\mathrm{GeV}$ range with emphasis on high resolution energy measurements of electrons, photons and muons. It is an effort involving a worldwide collaboration of 460 physicists belonging to 34 institutions from 13 countries. The preparation of the experiment took eight years from its conception to the beginning of data taking in summer 1989. The total cost was 200 MSf and 1100 technical man years. The detectors are installed within a 7800-t magnet providing a $0.5-\mathrm{T}$ field. We choose a relatively low field in a large volume to optimize muon momentum resolution, which improves linearly with the field but quadratically with the track length. From the interaction point outwards, the following detectors are installed (fig. 1):

- A central detector, tracking charged particles with a $50-\mu \mathrm{m}$ average single wire accuracy in the bending plane and with $450-\mu \mathrm{m}$ double track resolution. In the nonbending plane, the $Z$ coordinates are mea-

a) Metareg.

b) Formerly C.S. Draper Laboratory.

c) Also LAA.

d) Also Bologna.

e) Also F Bitter National Magnet Laboratory.

ก Also KEK.

g) Also Nanjing.

+ Deceased.

* Supported by the German Bundesministerium für Forschung and Technologie.

* * Partly supported by the grant CCA-8411/129 from the "US-Spain joint committee, Science and Technclogy program". sured by four layers of additional wire chambers providing $300-\mu \mathrm{m}$ single track resolution and $7-\mathrm{mm}$ double track resolution.

- An electromagnetic calorimeter using a new type of crystals (BGO), to measure energies of photons and electrons with an accuracy of $5 \%$ at $100 \mathrm{MeV}$ and better than $1 \%$ above $2 \mathrm{GeV}$.

- A hadron calorimeter, measuring hadron energies with $(55 / \sqrt{E}+5) \%$ resolution and $\Delta \Theta=2.5^{\circ}, \Delta \Phi=$ $3.5^{\circ}$ for jets, which also provides a clean muon sample by absorbing hadrons close to the $\mathrm{e}^{+} \mathrm{e}^{-}$interaction point, thus minimizing in-flight pion decays. and by tracking muons through the uranium absorber. The forward-backward part of the hadron calorimeter is specially designed for quick assembly and removal to provide access to the rest of the detectors.

- A muon detector, comprised of large drift chambers able to measure the sagitta of muon tracks to privide $\Delta p / p<1.5 \%$ at $p=50 \mathrm{GeV}$.

The detectors are complemented by a luminosity 

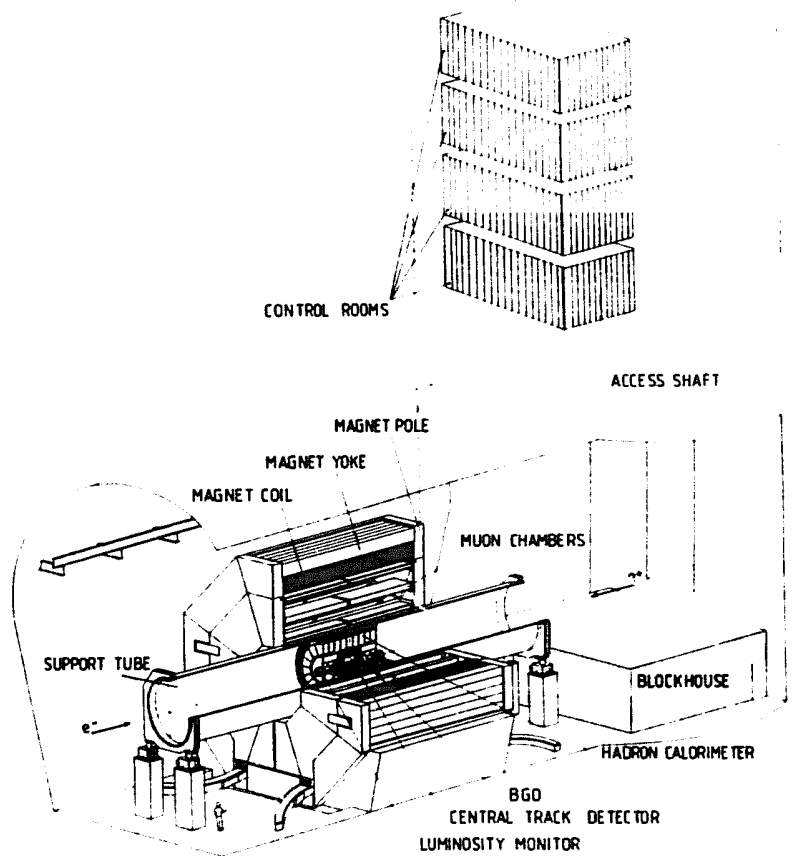

Fig. 1. Perspective view of the L3 experiment.

monitor, triggering and data taking electronics, a cluster of online computers and a mainframe computer for offline analysis. Ethernet and packet-switching networks are used for local and long-distance communications.

\section{General description of the $\mathbf{L 3}$ experiment}

\subsection{Detector}

The L3 experiment [1] is installed at interaction point 2 of the LEP $\mathrm{e}^{+} \mathrm{e}^{-}$storage ring. A 7800-t octagonally shaped solenoid houses all the detectors. The poles of the magnet are split into doors to give access to the field volume. The maximum field is $0.5 \mathrm{~T}$ and the effective field volume is $11.4 \mathrm{~m}$ across the flats of the octagonal aluminum coil and $11.9 \mathrm{~m}$ long. A watercooled screen separates the coil from the detector volume. The magnet rests on a concrete cradle integrated into the hall foundation (fig. 2).

The detectors are supported by a 32-m long and 4.45-m diameter steel tube which rests at both extremities on adjustable jacks placed on concrete pillars (fig. 3). The tube is concentric with the LEP beam line and symmetric with respect to the interaction point; it is mechanically coupled to the elements of the low- $\beta$ insertion, allowing alignment of all L3 detectors relative to the LEP beam.

The muon spectrometer forms three concentric chamber layers around the beam. It consists of two ferris wheels. each weighting $86 \mathrm{t}$. Each of the ferris wheels has eight octants and each octant has five chambers. The muon spectrometer covers $76 \%$ of the solid angle.

The central section of the support tube houses the inner detectors, arranged as "barrel" elements around

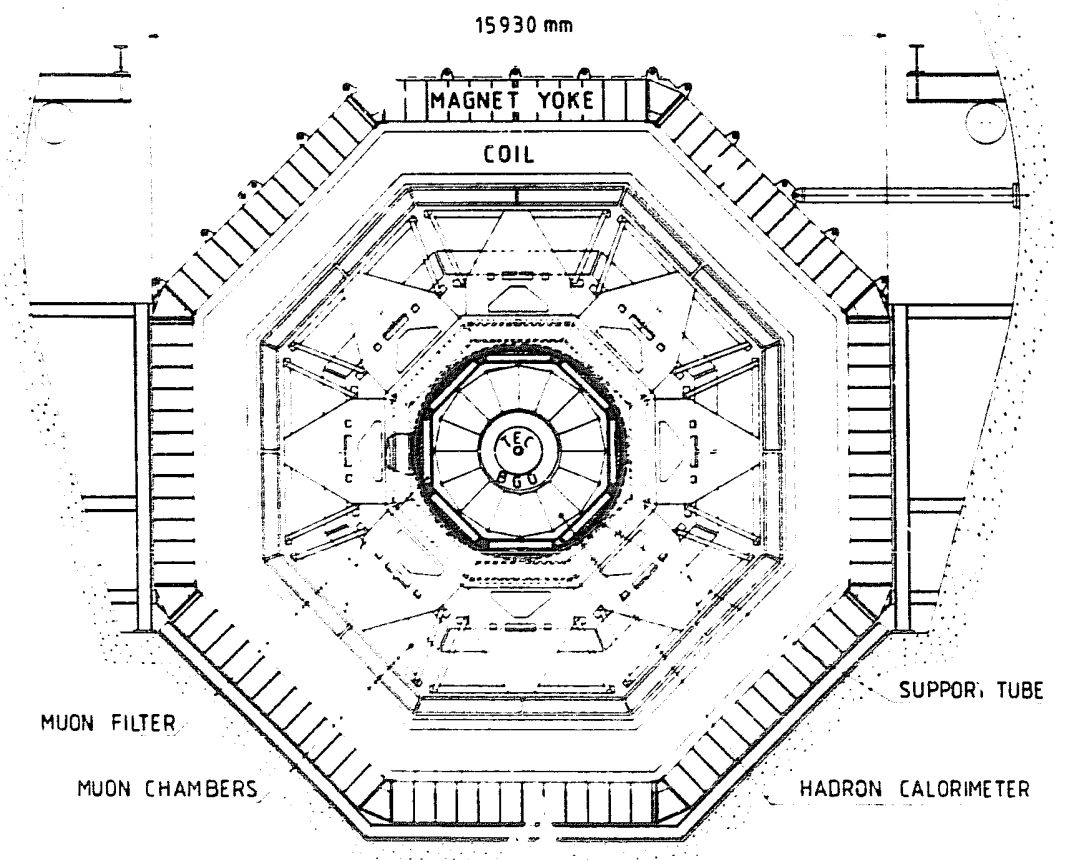

Fig. 2. Transverse view of $\mathrm{L} 3$. 


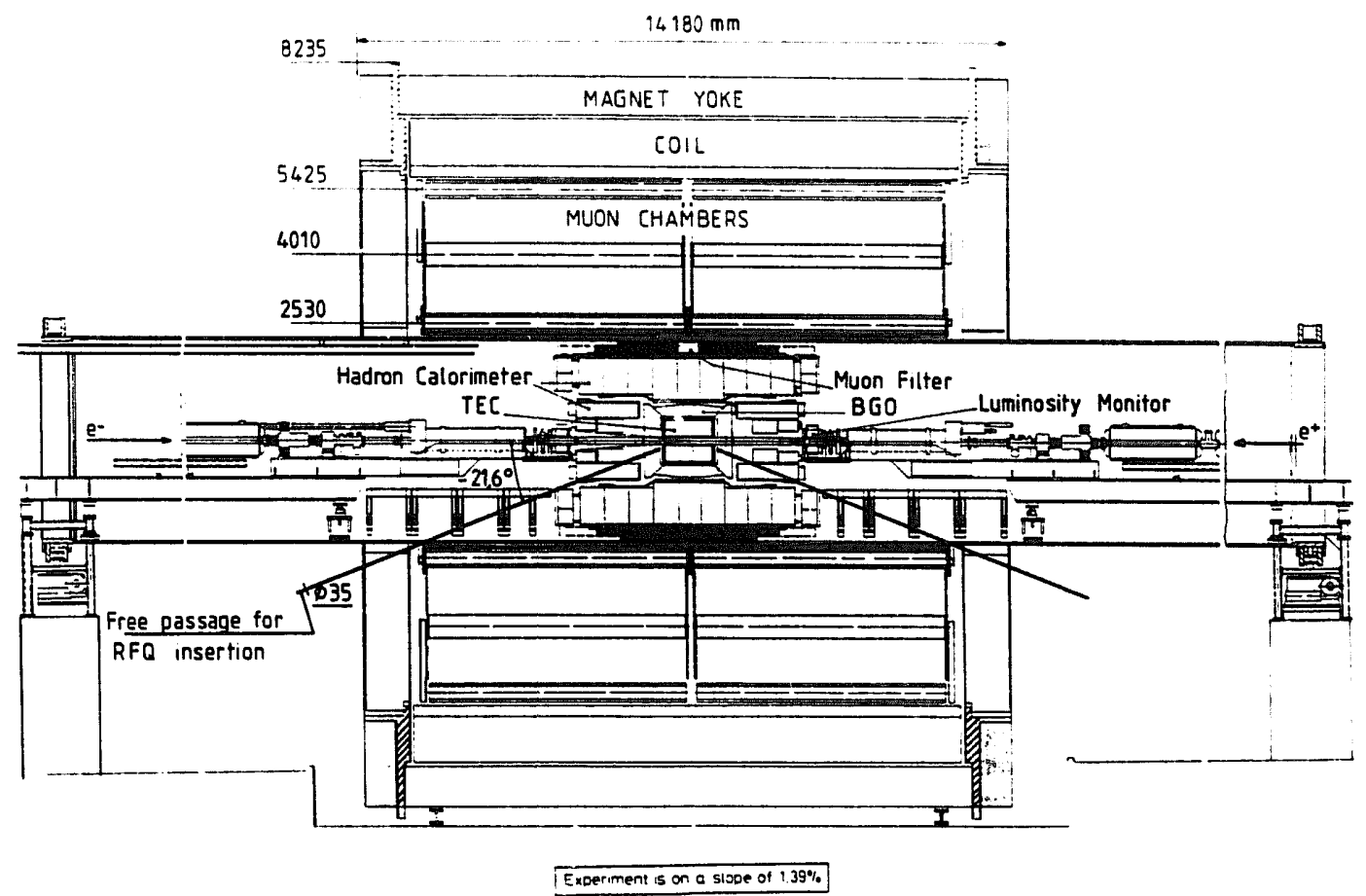

Fig. 3. Longitudinal view of L3.

the beam pipe and as "end cap" elements in the forward and backward directions. The barrel elements consist of Muon Filter, Hadron Calorimeter Barrel, Barrel Scintillators, BGO Electromagnetic Calorimeter, Vertex Chamber and the beam pipe. The Luminosity Monitors are situated immediately in front of the low- $\beta$ magnets.

\subsection{Experimental area}

The 21.4-m diameter, $26.5-\mathrm{m}$ long experimental hall is oriented longitudinally with respect to the LEP beam line, which enters the hall with a slope of $1.39 \%$. The hall is equipped with a $40-t$ overhead crane, an 11-t monorail fixed to the ceiling and a $10-\mathrm{t}$ jib arm mounted on the back cavern wall. There is also a 14-t gantry crane inside the support tube.

The 23-m diameter and 52-m deep access shaft connected to one end of the experimental hall serves as access for experimental equipment and personnel, for hall services (water, cables, gas, ventilation) and provides in its upper half space for four counting rooms with a total area of $325 \mathrm{~m}^{2}$ directly above the LEP beam line and protected from radiation by a $1.7-\mathrm{m}$ thick shielding of concrete beams. A "blockhouse" at the hottom of the access shaft provides shielded space for electronics near the detectors (fig. 1).

The detector electronics is powered via a dedicated

Table 1

L3 detector gas systems

\begin{tabular}{|c|c|c|c|c|c|c|c|}
\hline Detector & Mixture & $\begin{array}{l}\text { Detecior } \\
\text { volume } \\
{\left[\mathrm{m}^{3}\right]}\end{array}$ & $\begin{array}{l}\text { Operating, } \\
\text { pressure } \\
\text { [bar gauge] }\end{array}$ & $\begin{array}{l}\text { Fresh gas } \\
\text { flow } \\
{\left[\mathrm{m}^{3} / \mathrm{h}\right]}\end{array}$ & $\begin{array}{l}\text { Recircula- } \\
\text { tion flow } \\
{\left[\mathrm{m}^{3} / \mathrm{h}\right]}\end{array}$ & $\begin{array}{l}\text { Number } \\
\text { of } \\
\text { parallel } \\
\text { circuits }\end{array}$ & $\begin{array}{l}\text { Special } \\
\text { requirements } \\
\text { [Relative } \\
\text { stability] }\end{array}$ \\
\hline Time expansion chamber & $\mathrm{CO}_{2}+20 \%$ i-butane & 0.75 & 1 & $\begin{array}{l}\text { discontin- } \\
\text { uous }\end{array}$ & 0.2 & 1 & $\begin{array}{l}\mathrm{O}_{2}<1 \mathrm{ppm} \\
\Delta \text { drift v. }< \pm 0.1 \%\end{array}$ \\
\hline Central $Z$ chambers & $\mathrm{AR}+20 \% \mathrm{CO}_{2}$ & 0.05 & 0.005 & 0.02 & 0 & $2 \times 2$ & \\
\hline Muon filter & $\mathrm{AR}+20 \% \mathrm{CO}_{2}$ & 3 & 0.005 & 0.24 & 0 & $16 \times 10$ & \\
\hline LM forward chambers & $\mathrm{AR}+20 \% \mathrm{CO}_{2}$ & $<0.01$ & 0.005 & $<0.02$ & 0 & $2 \times 2$ & \\
\hline Hadron calorimeter end caps & $\mathrm{AR}+20 \% \mathrm{CO}_{2}$ & 1 & $0.01-0.05$ & 0.06 & 0 & $4 \times 7$ & $\Delta$ density \\
\hline Hadron calorimeter barrel & $\mathrm{AR}+20 \% \mathrm{CO}_{2}$ & 16 & $0.01-0.05$ & 0.8 & 0 & $16 \times 9 \times 6$ & $< \pm 0.05 \%$ \\
\hline Muon $p$ chambers & AR + 38.5\% ethane & 250 & 0.001 & 2.5 & 50 & $16 \times 5$ & $\Delta$ mix. $< \pm 0.1 \%$ \\
\hline Muon $Z$ chambers & $\mathrm{AR}+8.5 \%$ methane & 50 & 0.001 & 0.5 & 10 & $16 \times 3$ & \\
\hline
\end{tabular}


2-MV A transformer and cooled by water/air heat exchangers mounted inside the racks. In addition, each counting room is equipped with two $25-\mathrm{kW}$ air conditioners. The ventilation system of the experimental area has a normal capacity of $40000 \mathrm{~m}^{3} / \mathrm{h}$, stabilizing the temperature to $\pm 1{ }^{\circ} \mathrm{C}$ with a dew point at $12^{\circ} \mathrm{C}$. In an emergency, the capacity of the ventilation system can be doubled.

A $1780-\mathrm{m}^{2}$ surface hall equipped with a $65-\mathrm{t}$ overhead crane and a 16-m wide, 8-m high door covers the vertical access shaft. It is used as a test and assembly hall for all equipment to be lowered into the experimental area. It also houses the magnet power supply, the electricity and water distribution and the ventilation for the experimental installations. To permit on-site assembly of large and heavy parts of the experiment, a $1420-\mathrm{m}^{2}$ assembly hall was built near the vertical access shaft. It is equipped with a $65-t$ traveling gantry crane.

\subsection{Gas systems}

Special gas systems have been built for the different wire detectors used in L3 (table 1). Recirculation is used where possible to keep gas cost low, for better control of impurities and for leak detection by balancing overall input and output. A very good lorig-term stability of the mixture ratio is achieved by using infrared analyzers with regular recalibration against a reference mixture.

\subsection{General safety and gas safety}

The design, construction and installation of L3 followed the general CERN saf ty rules. In addition, special restrictions are applicable to underground experimental halls with limited access (in particular minimization of flammable material and of halogenated plastics). At an early design stage, the L3 collaboration made various studies to limit the use of flammable hydrocarbons in its detectors. A considerable reduction of fire risk was obtained by replacing, for the uranium calorimeter, the initially planned mixtures with high concentrations of n-pentane by the inert argon/ $\mathrm{CO}_{2}$ mixture. However, to achieve the required performance in the precision muon chambers, the ethane concentration could not be reduced below $38.5 \%$, resulting in the use of slightly more than $100 \mathrm{~kg}$ of hydrocarbons in L3. This choice was accepted by CERN Safety in view of a whole package of complementary safety features, such as all-metal detector walls, double gaskets, fusing of electric power, sensitive leak detection, recirculating gas systems with sensitive oxygen alarms and a dedicated ventilation system for the detector volumes. The latter is combined with a system of smoke detection and a facility for injecting sufficient quantities of inert gas to stop any fire inside the detector.

\section{The magnet}

\subsection{General description of the magnet}

All the L3 detectors are mounted inside the huge solenoid coil which is surrounded by an iron yoke and closed at its ends by two poles equipped with hinged doors. The main parameters of the magnet are listed in table 2.

\subsection{The coil}

The coil is made of industrial plates welded together on the CERN site. The selected material, "Anticorodal 041 ", with heat treatment " 71 ", has $6 \%$ less conductivity than pure aluminum but better mechanical and welding properties. Cooling is provided by two independent circuits made of "Extrudal 050", an aluminum alloy with high resistance to corrosion, welded onto the inner and outer edges of the coil (fig. 4). The interturn insulation is made of $10-\mathrm{mm}$ fiber glass plates covered with $0.2-\mathrm{mm}$ of Mylar. The $30-\mathrm{kA}$ current is a compromise between the production capabilities of the aluminum supplier (dimensions, plates thickness, flatness tolerances), the investment in handling tools and manpower to manufacture the coil as well as the difficulties inherent to the transport of high currents. The 168 turns coil is divided in 28 packages which are bolted together. The mechanical rigidity of each package is insured by axial bolts. All bolts are triple insulated. The gaps between turns are closed with rubber joints to minimize heat transfer to the detector. The packages rest on insulated bronze skates which follow the thermal expansion by moving on two rails embedded in the lower part of the magnet yoke. The coil is fastened to one pole, whereas at the other pole, electrically insulated air springs permit coil motion arising from thermal expansion. An active thermal shield placed on the inside of the coil protects the detectors.

Table 2

Main parameters of the magnet

\begin{tabular}{ll}
\hline Inside radius of the coil & $5930 \mathrm{~mm}$ \\
Width of the conductor & $890 \mathrm{~mm}$ \\
Outside radius of the yoke & $7900 \mathrm{~mm}$ \\
Total length of the coil & $11900 \mathrm{~mm}$ \\
Electrical power at the taps & $4.2 \mathrm{MW}$ \\
Central field & $0.5 \mathrm{~T}$ \\
Stored magnetic energy & $150 \mathrm{MJ}$ \\
Rated current & $30 \mathrm{kA}$ \\
Current density in the conductor & $55.5 \mathrm{~A} / \mathrm{cm}^{2}$ \\
Cooling water & $150 \mathrm{~m}^{3} / \mathrm{h}$ \\
Coil weight (aluminum) & $1100 \mathrm{t}$ \\
Shielding weight (soft iron) & $6700 \mathrm{t}$ \\
\hline
\end{tabular}




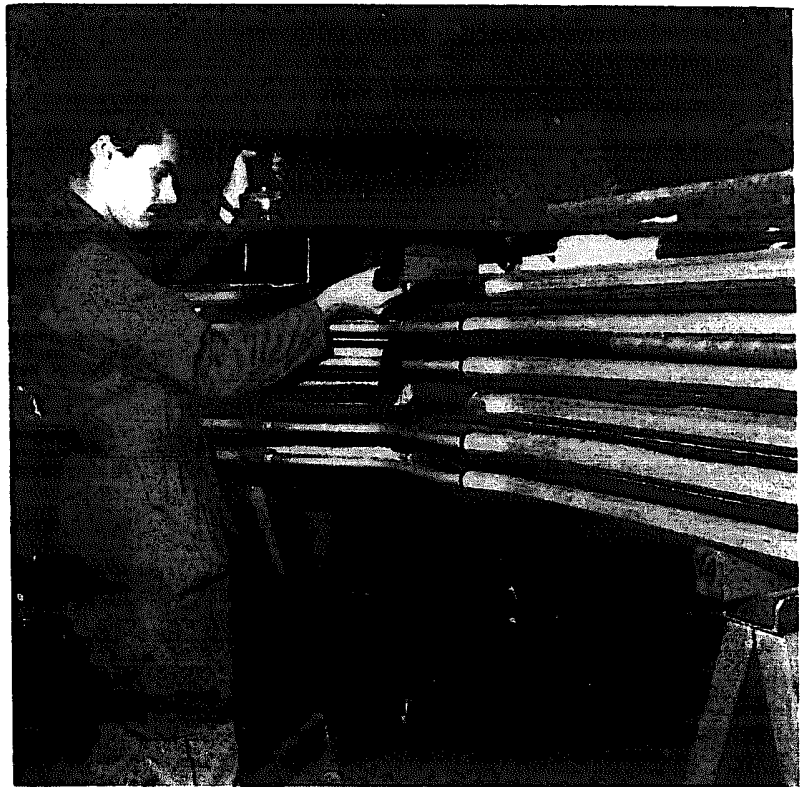

Fig. 4. Edge-cooled plate for the magnet coil.

The manufacturing procedure of the coil has been determined by the dimensions of the detector. The sectors were welded using electron beam technology. A set of special tools centered on a welding gun of $45 \mathrm{~kW}$ at $50 \mathrm{kV}$ (fig. 5) was developed to support the quasi-industrial production. In a first step, four sectors were welded together, forming half-turns of $3.2 \mathrm{t}$ (figs. 6 and 7). After completion of this work, the equipment was moved from the CERN SPS site to the experimental site where, by welding 12 half-turns together, the unit weight

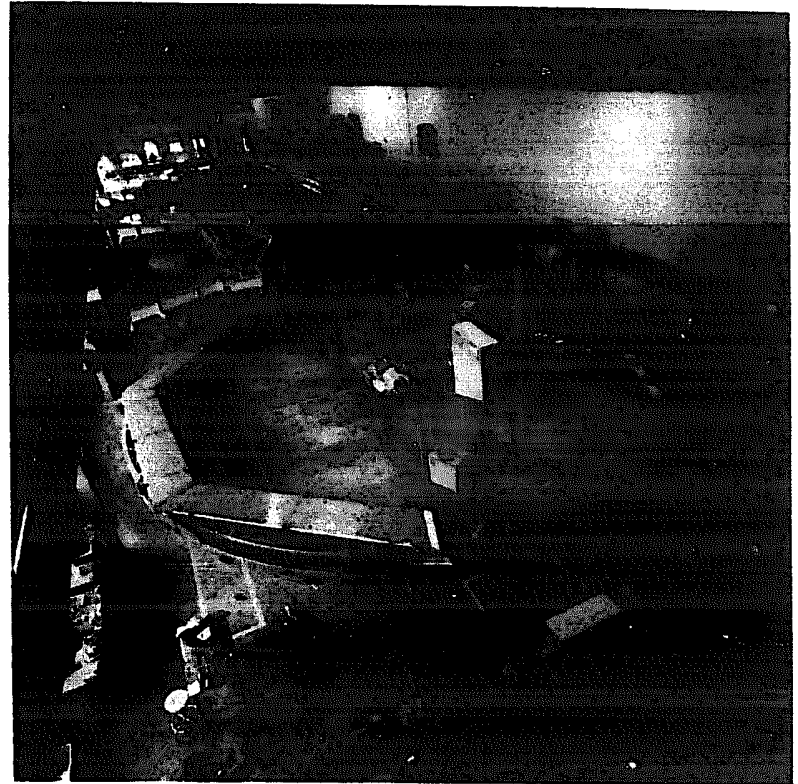

Fig. 6. First coil workshop, assembling plates into half-turns.

was brought to $38 \mathrm{t}$ (figs. 8-10). Each 6-turn package has four cooling circuits; this design is a compromise between the ideal case of having two circuits per turn but an enormous amount of piping or two circuits per package but water pressure problems. The cooling circuits include more than 6000 welded joints. Numerous checks concerning dimensions, thermal, mechanical, electrical, pressure and corrosion behavior have been conducted during the manufacturing period.

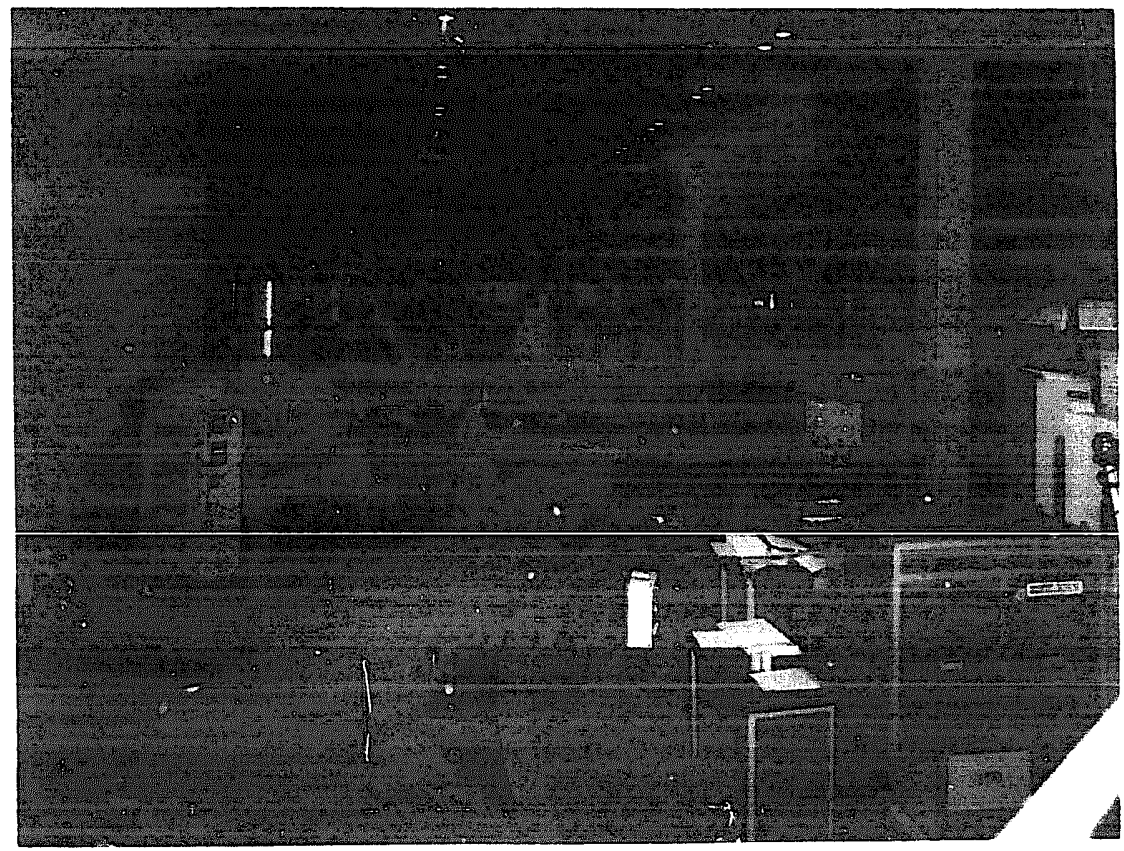

Fig. 5. The 45-kW electron beam welding gun. 


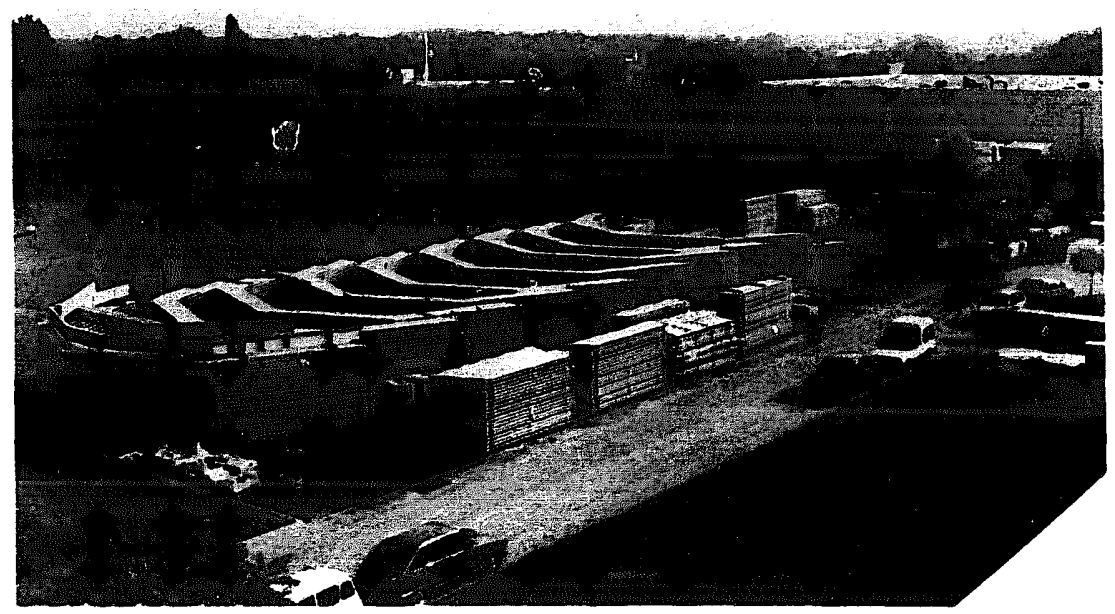

Fig. 7. Stacks of half-turns.

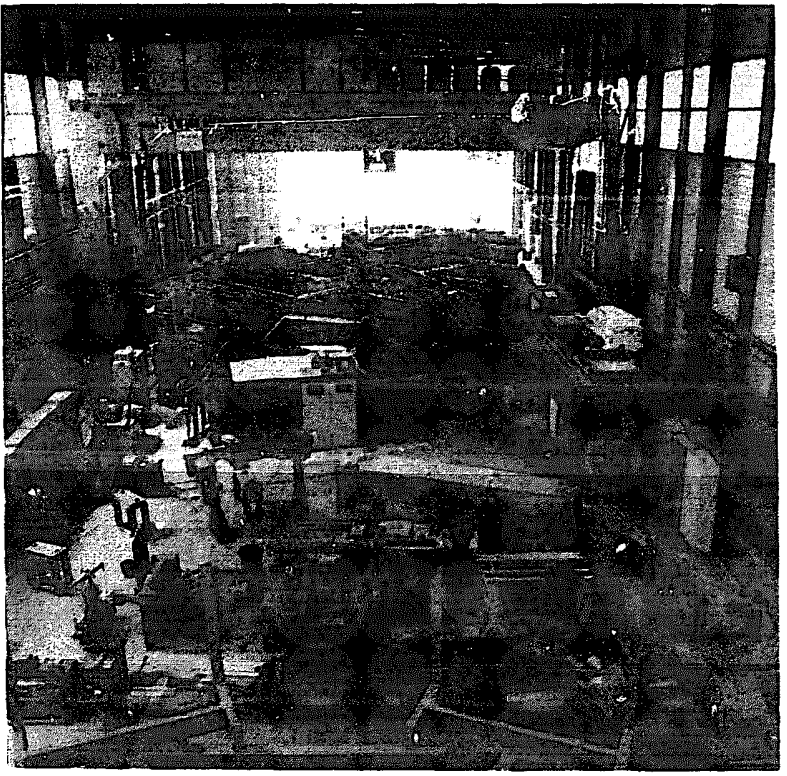

Fig. 8. Second coil workshop, assembling half-turns into 6-turn packages.

\subsection{The magnetic structure}

The magnetic structure is made of soft iron with $0.5 \%$ carbon content. The poles are made of $1100 \mathrm{t}$ of self-supporting steel structure (fig. 11) giving the required rigidity and serving as a support and reference frame to mount the $5600 \mathrm{t}$ of filling material, which provides the mass needed for the magnetic flux return both in the poles and in the barrel. The filling material supplied by the USSR is made of $50-\mathrm{mm}$ and $40-\mathrm{mm}$

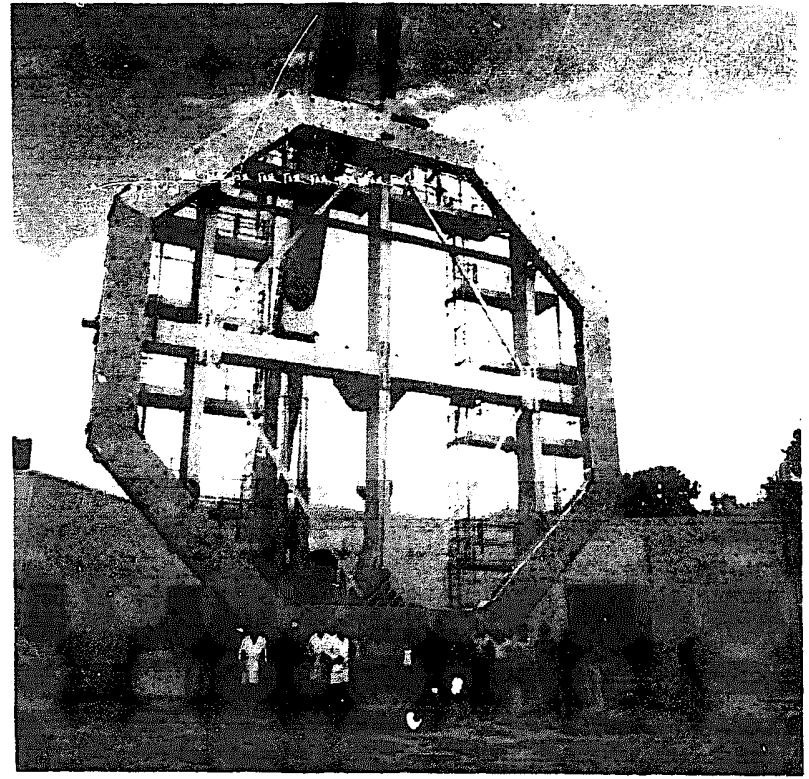

Fig. 9. Lifting the first 6-turn package.

thick soft iron plates cut to shape and tack-welded to form individual masses of about $40 \mathrm{t}$ for the barrel (fig. 12) and $15 \mathrm{t}$ for the poles.

A pole consists of two parts, the crown and the double doors (fig. 13). All the parts are made of open frames bolted together and positioned with expansion keys. The crown forms a complete ring and each door a half-ring. The frame elements of the doors are welded together in situ. Two rails on each side of the open frames are used to guide the stacks of filling material. 


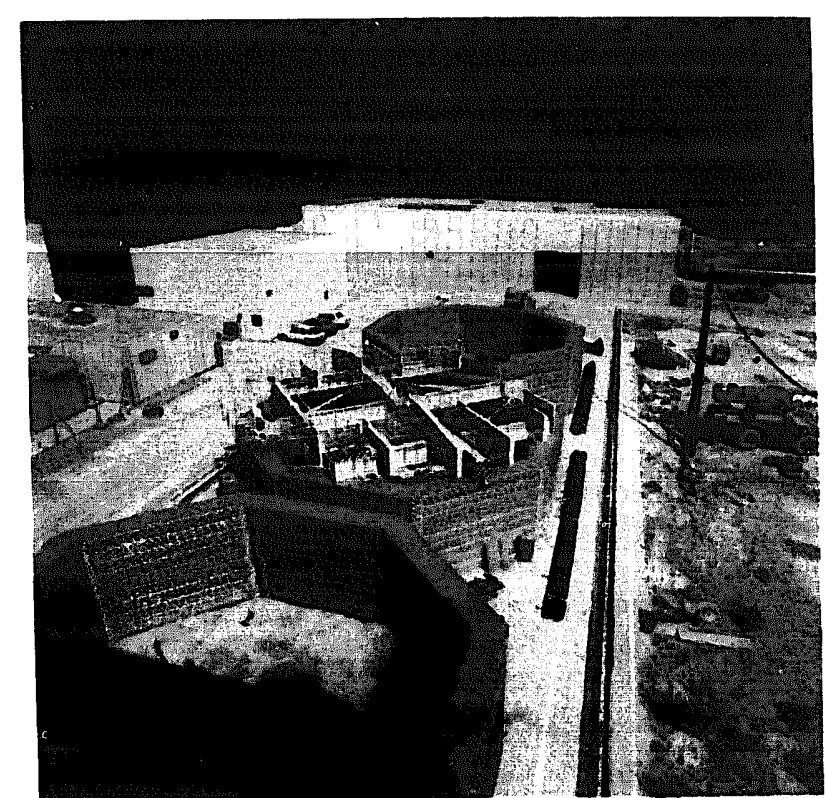

Fig. 10. Stacks of finished 6-turn packages.

Each filled half door weights $340 t$, rests on grease skates positioned under the center of gravity and rotates around large hinges (fig. 14). The hinges can be mechanically disconnected from the doors to prevent overstressing due to the magnetic pressure on the poles.

\subsection{The bus bars and power supply}

The power supply is installed in the surface hall and connected to the magnet through the shaft with a set of

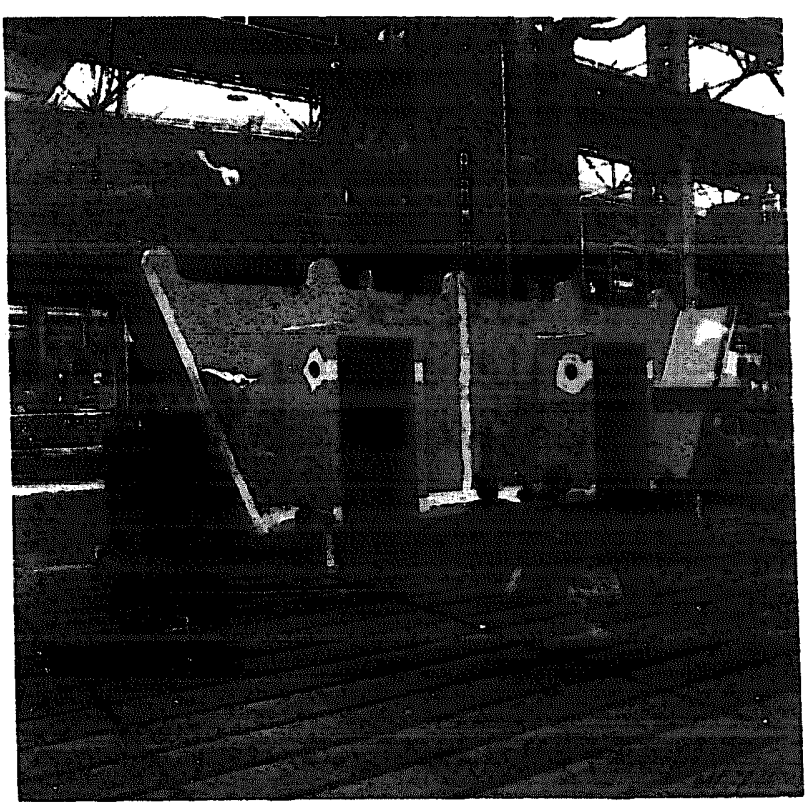

Fig. 11. A steel skeleton for a door.

82-m long water-cooled bus hars made of $30 t$ of aluminum tubes. To reduce the fringe field and the radiated electrical noise, both polarities of the bus bars are interleaved. The power supply is a thyristor converter delivering a maximum current of $31.5 \mathrm{kA}$ at 150 $\mathrm{V}$. It consists of two transfoimers followed by six banks of water-cooled thyristors equipped with passive filters and $\mathrm{fr}$-wheel diodes. During the magnet tests, the current was stabilized within $\pm 0.5 \%$ of the rated value. Precision and reproducibility will be improved by the

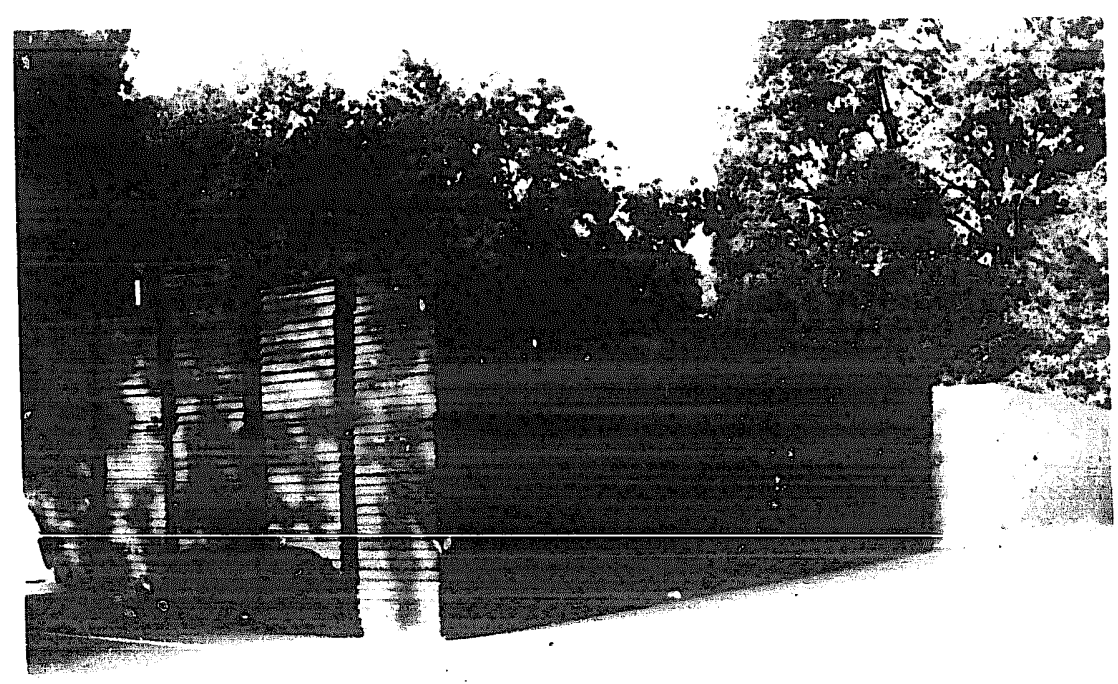

Fig. 12. Filling material. 


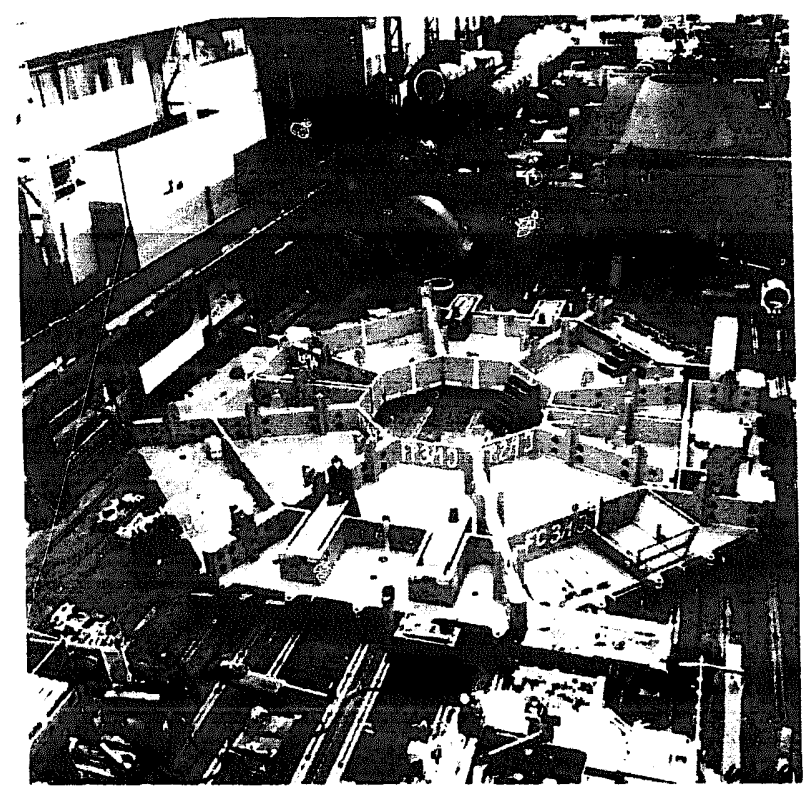

Fig. 13. One of the magnet poles (door and crown) during trial assembly.

addition of an NMR probe. The magnet coil is grounded in the middle through a resistance of $1 \Omega$.

\subsection{The magnet monitoring system}

The magnet system includes 159 cooling circuits and 29 interior electrical connections, all monitored by embedded detectors. In addition, potential and field monitoring devices, water flow meters and control valves are checked (about 2000 detector signals in total). The

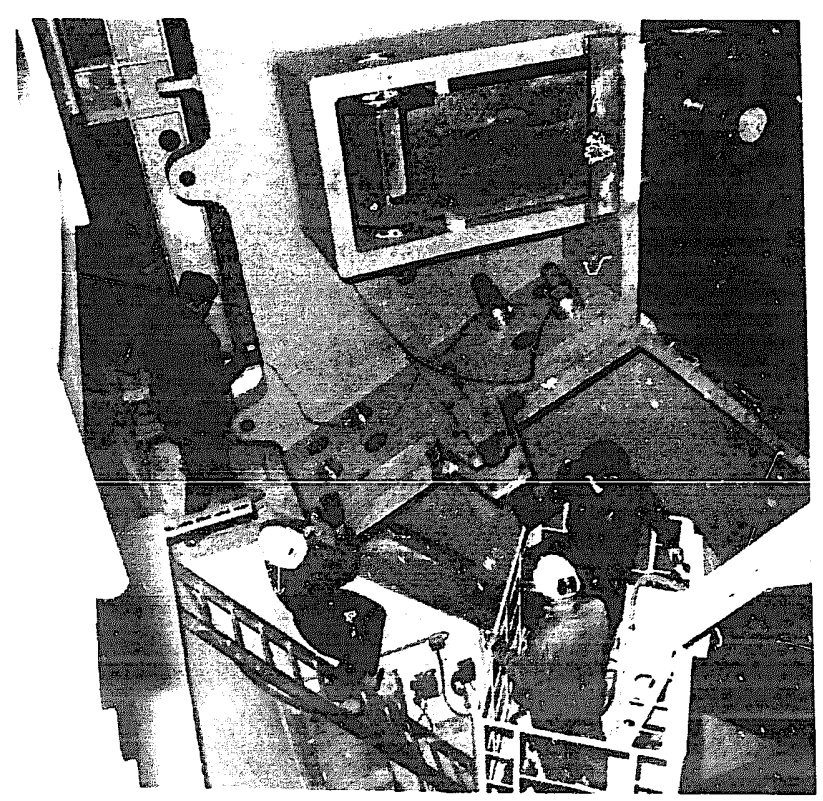

Fig. 14. View of a magnet door hinge. magnet monitor ng system placed on the plug of the vertical shaft feeds general information concerning the magnet behavior into the L3 slow control system.

\subsection{Field measurement}

Because of the large volume, which is furthermore partially obstructed by the support tube, the field measurement has been divided into two parts: The inner volume of the support tube was mapped with Hall plates [2]. The remaining volume has been mapped with about a thousand magnetoresistors, permanently installed on the muon chambers. In addition, five NMR probes monitor the absolute value of the field.

For the interior of the support tube, a mapping device was used which had been developed to map the field of all four LEP experiments. Two rods, each equipped with 60 Hall plates, rotate around the central axis. The rotating mechanism is supported by the two rails on which the hadron calorinieter rests, removed during the measurements. Both the movement in the azimuthal as well as in the axial direction are under remote control. The Hall plates are oriented in the $Z$ (main component), $r$ and $\Phi$ directions. Each component is measured twice. The relative alignment within a pair and to other pairs is known with an accuracy of 0.2 mrad. This allows one to determine the minor components of the field with the "sum of differences" algorithm even in the presence of a misalignment of the gea: [2].

The volume occupied by the muon chambers has been mapped with magnetoresistors (main component only). Magnetoresistors were chosen because they are economica!, stable in time and (since they are sensitive to $B^{2}$ ) need only to be calibrated for one polarity of the field. Their temperature dependence was taken care of by adding compensating resistors in parallel. The distribution of the magnetoresistors on the muon chambers was done in such a manner as to have a measurement whenever the value of the main component changes by $40 \mathrm{G}$. To facilitate their installation on the muon chambers, up w ten magnetoresistors were mounted in a chain in alurininum housings up to $1 \mathrm{~m}$ long. These magnetoresistor ensembles were then calibrated in a small solenoid.

\subsection{The detector support structure}

The support tube (ST) is a $32-\mathrm{m}$ long, $50-\mathrm{mm}$ thick 4.45-m outer diameter heterogeneous tube (fig. 15) with a flange support at each end to transmit the load to the ground. The part of the ST which is inside the magnet $(14.1 \mathrm{~m})$ is of nonmagnetic stainless steel with a $4.6-\mathrm{m}$ long octagonal double walled central section (fig. 16). The remaining portion is of carbon steel. Each flange rests on two servo-controlled mechanical jacks to allow 


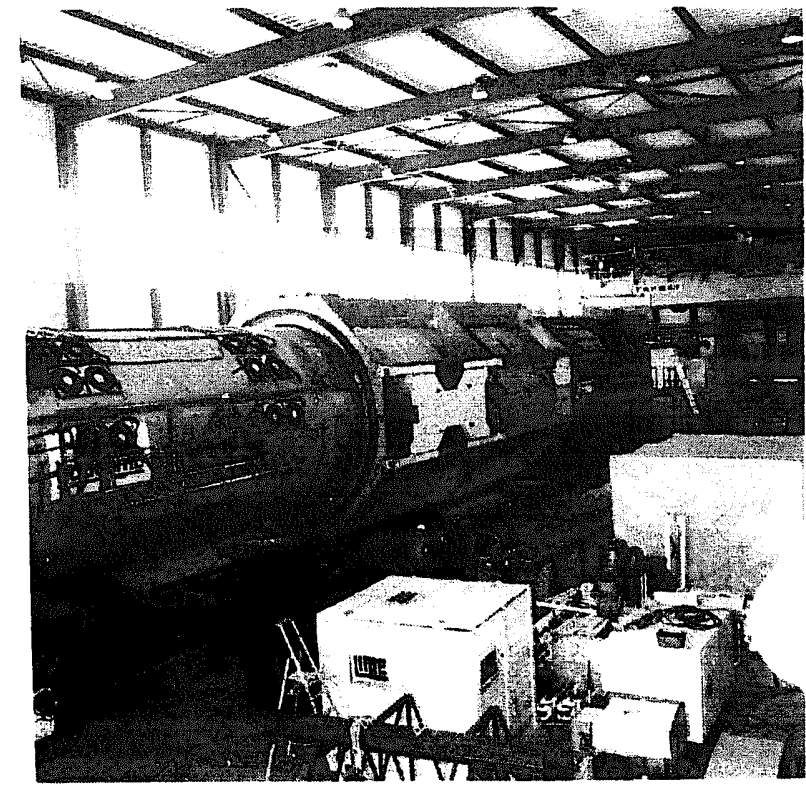

Fig. 15. The support tube, with the two captive torque tubes, in the assembly hall.

continuous alignment of the ST with respect to the LEP beam (fig. 17).

The muon chambers are supported by two torque tubes (TT) on rails attached to the exterior of the support tube. Each TT, made of nonmagnetic stainless steel, has a mass of $29.5 \mathrm{t}$ and supports eight $7-t$ octants of muon chambers. A TT is a cylindrical shell with octagonal ring flanges and eight webs extending from flange to flange on the outside of the shell (fig. 18). The

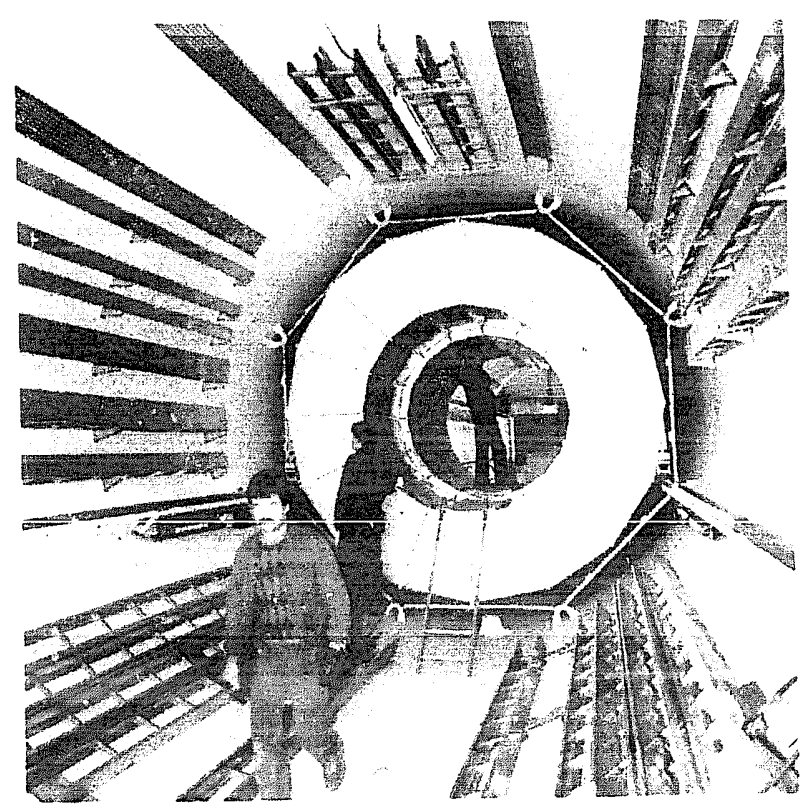

Fig. 16. Installation tests of the barrel hadron calorimeter inside the support tubes.

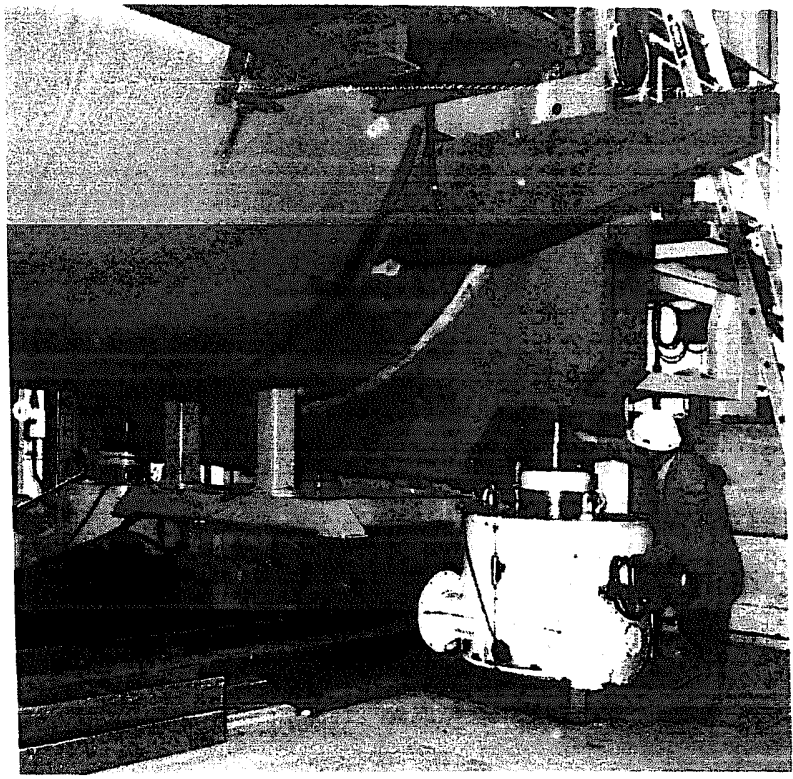

Fig. 17. One of the servo-controlled jacks.

flanges hold the octant end frames in a stable configuration while the shell resists the torsion of one flange with respect to the other. The two TT have been fitted over the cylindrical part of the ST, before the welding of the last flange support and are now captive on the ST (fig. 15). The finished ST/TT unit has thus a mass of $340 \mathrm{t}$. Stresses and deformation of the ST/TT assembly have been studied by the finite elements method. It was found that under full load the tube sags by $7 \mathrm{~mm}$.

\subsection{The magnet assembly}

The assembly of the magnet progressed in four phases:

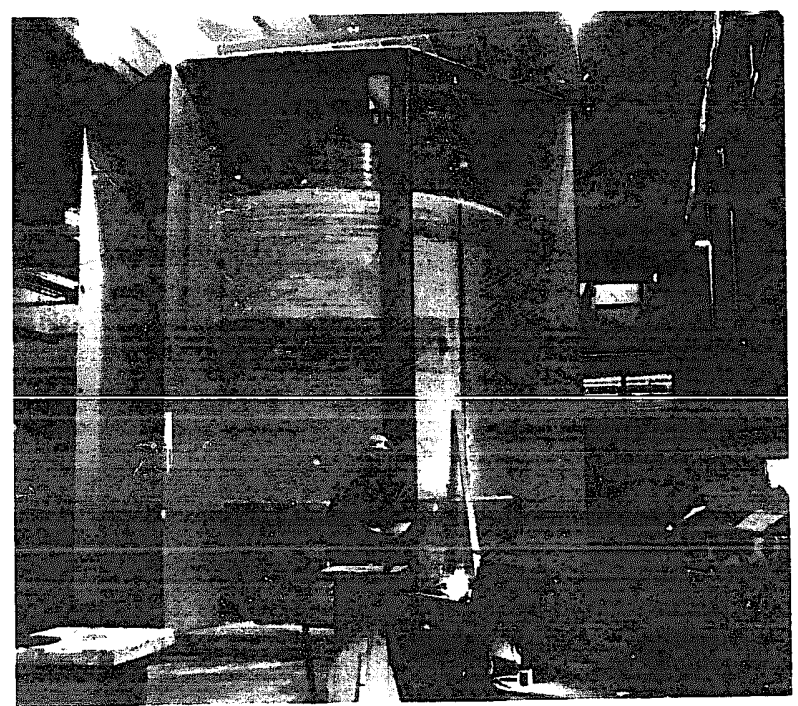

Fig. 18. View of a torque tube before machining. 


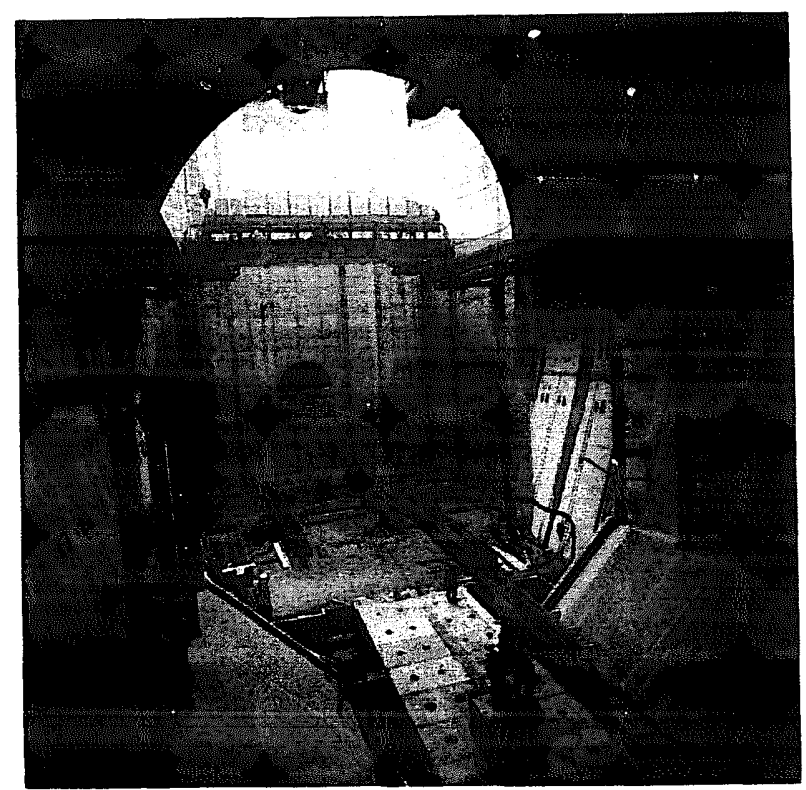

Fig. 19. The bottom of the hall serves as a cradle for the magnet.

a) First phase: the lower $3 / 8$ of the barrel and $5 / 8$ of the first crown were assembled and aligned with respect to the LEP beam to form the coil cradle, which is the reference suppori for the coil (figs. 19-21);

b) Second phase: the 28 coil subassemblies were mounted in this cradle, aligned with respect to the LEP beam and electrically connected in series by welding. The $5 / 8$ of the second crown, together with the two vertical walls of the barrel were then erected and the piping for the coil completed (figs. 22 and 23);

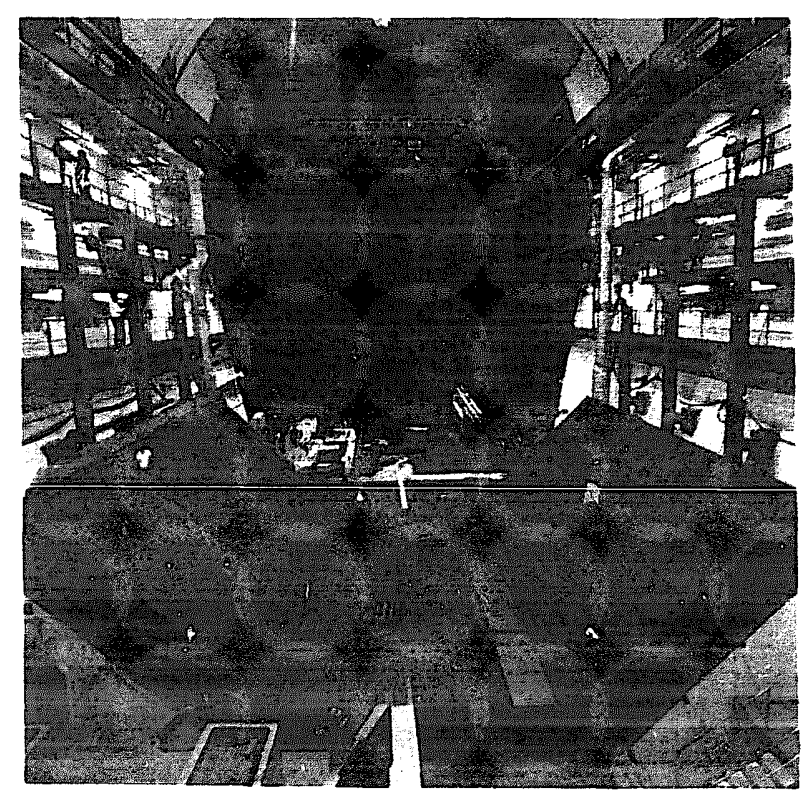

Fig. 20. 3/8 of the barrel installed in the cradle.

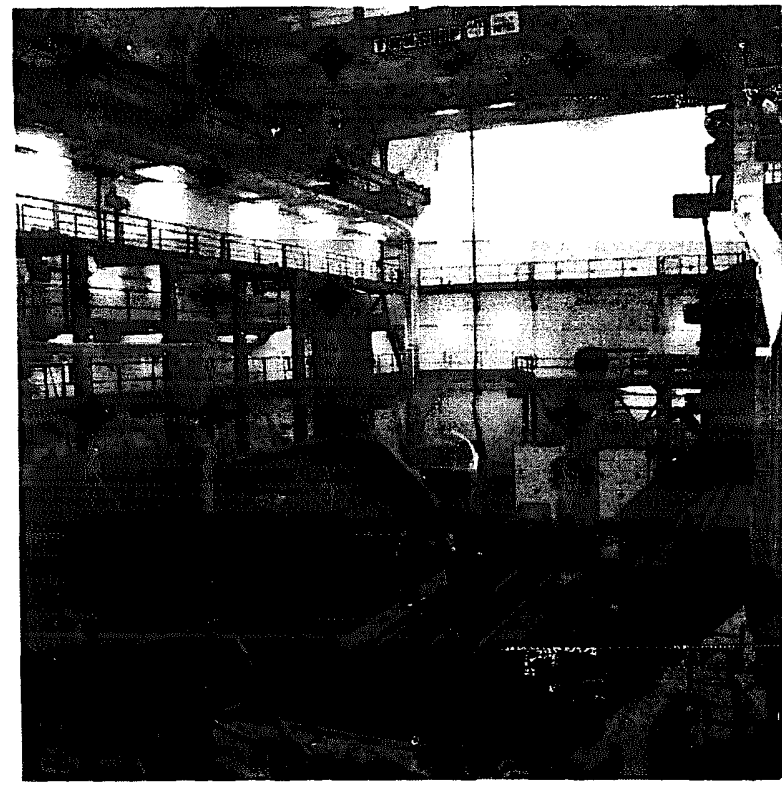

Fig. 21. 5/8 of a crown and $3 / 8$ of the barrel ready to receive the coil.

c) Third phase: the doors, the crown top arcs and barrel roof were mounted (fig. 24) and the door elements were welded together;

d) Fourth phase: filling of the two poles was completed (figs. 25 and 26).

After the magnet had been assembled and operated for $100 \mathrm{~h}$ at nominal current, the mounting of the ST/TT unit inside the magnet began. The ST/TT unit was pulled out of the assembly hall and lifted vertically (fig. 27) by a 1000-t crane helped by an $800-t$ foot

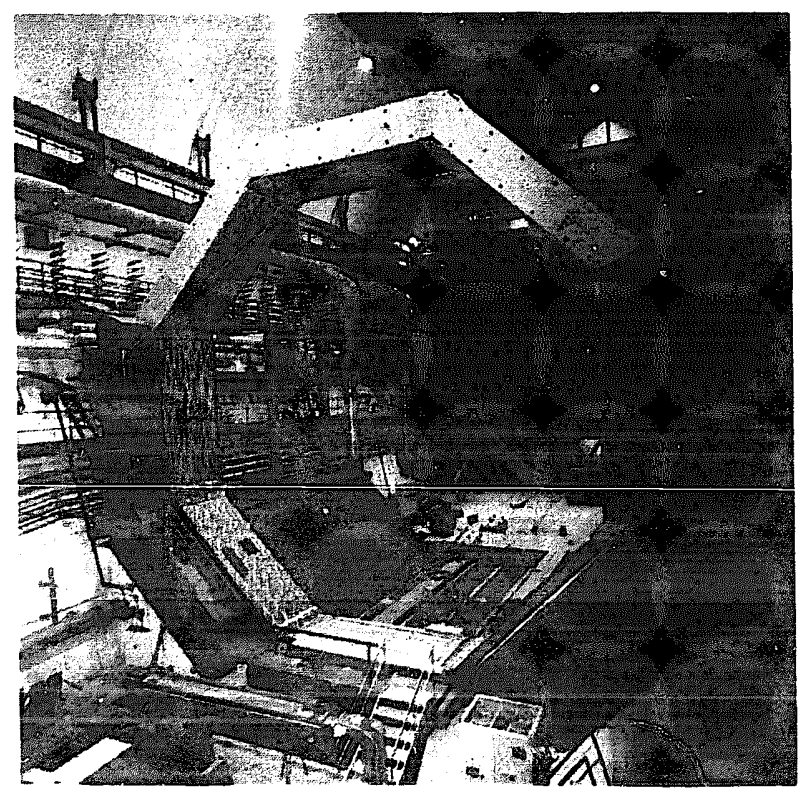

Fig. 22. The beginning of coil installations. 


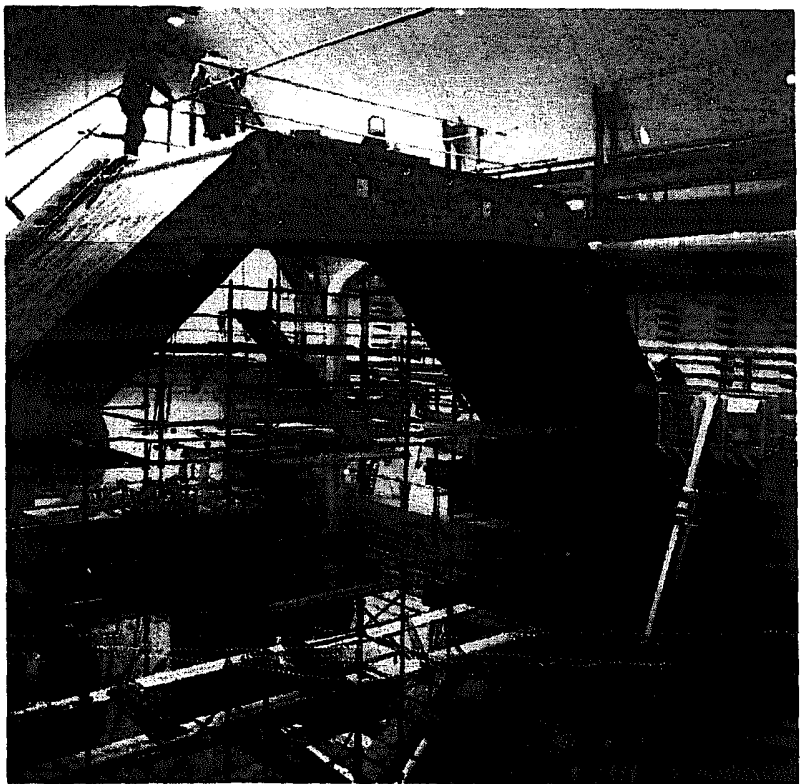

Fig. 23. The coil installation progresses.

crane; it was then brought over the opening in the roof of the building covering the vertical shaft, lowered through the shaft down to the experimental srea (fig. 28) and inserted into the magnet (fig. 29). The same crane was used two days later to lower the fully assembled 261-t hadron barrel calorimeter.

\subsection{Properties of the magnet}

The coil axis has been aligned to within $2 \mathrm{~mm}$ of the

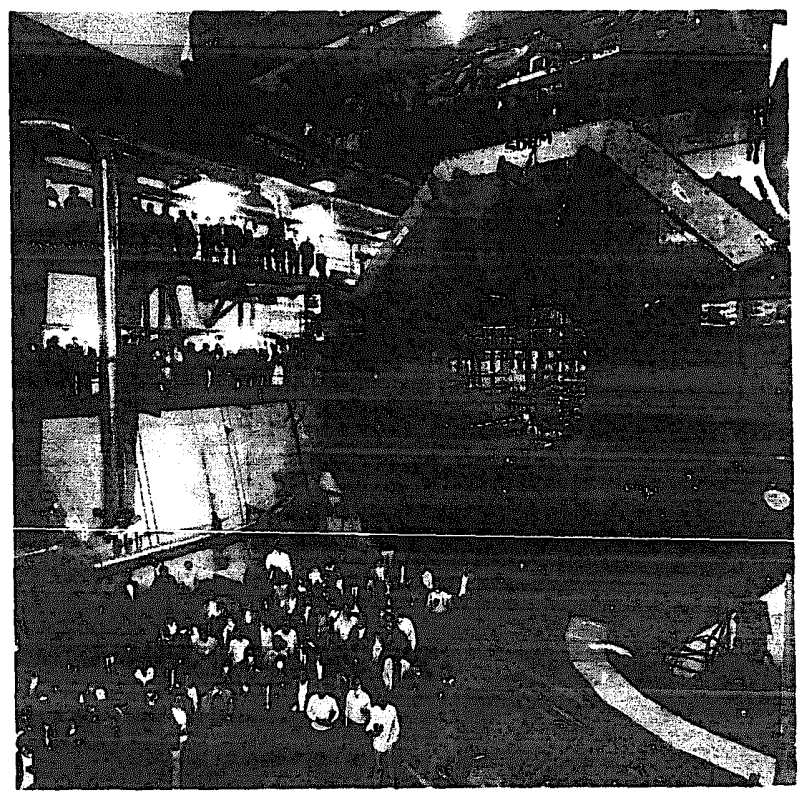

Fig. 24. Some of the L3 collaborators in front of the partially assembled second pole. The top of the coil is still visible.

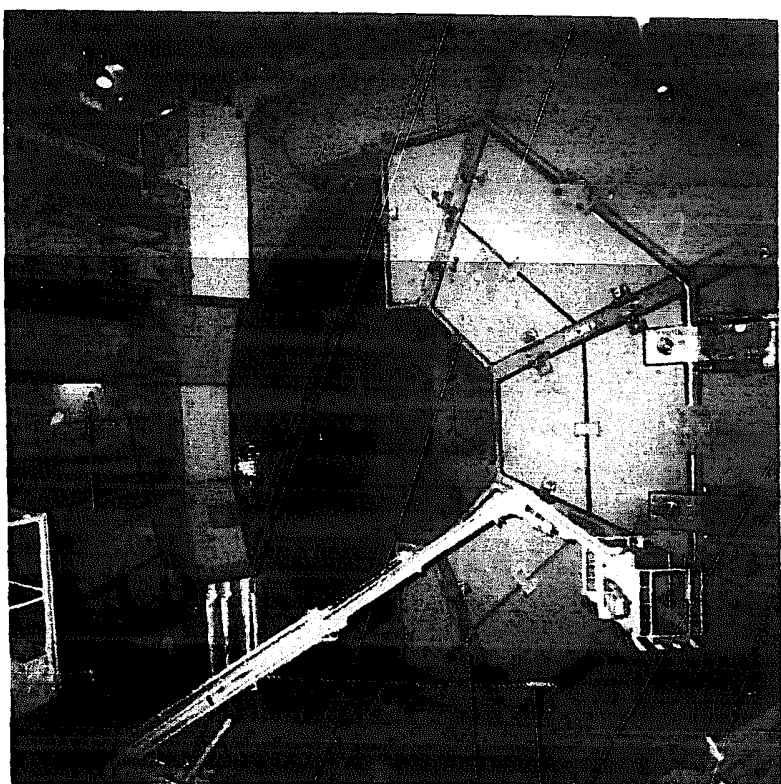

Fig. 25. The filled magnet doors.

beam axis (fig. 30). The measured field of the magnet (fig. 31) agrees with the design value.

\section{Muon detector}

\subsection{Design considerations}

The L3 muon detector has been designed [1] to measure high energy muons to an accuracy of $\Delta p / p=$ $2 \%$ at $50 \mathrm{GeV}$, thus providing a $1.4 \%$ dimuon mass

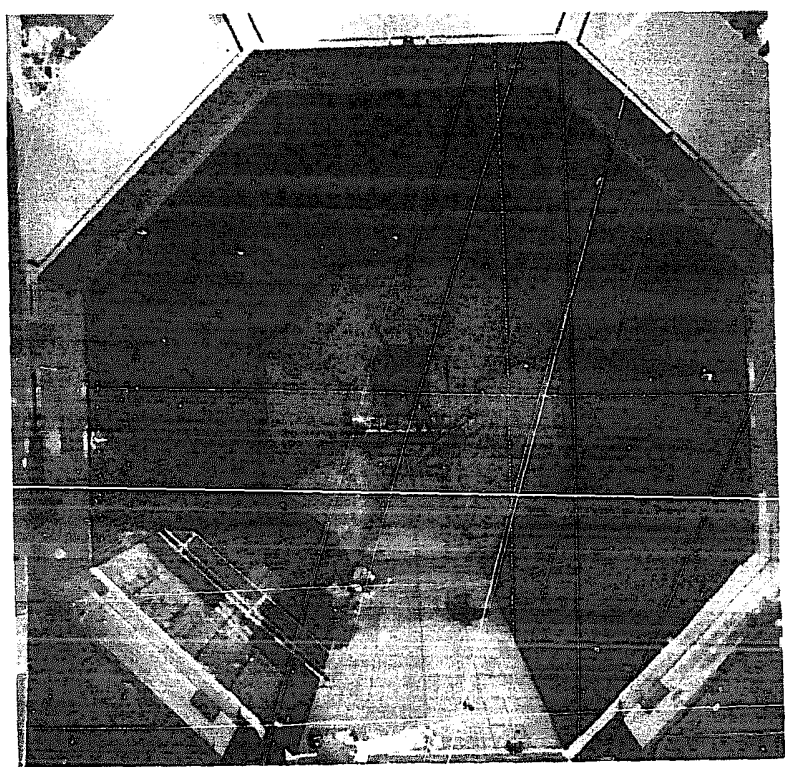

Fig. 26. The completed magnet. Notice the heat shield. 


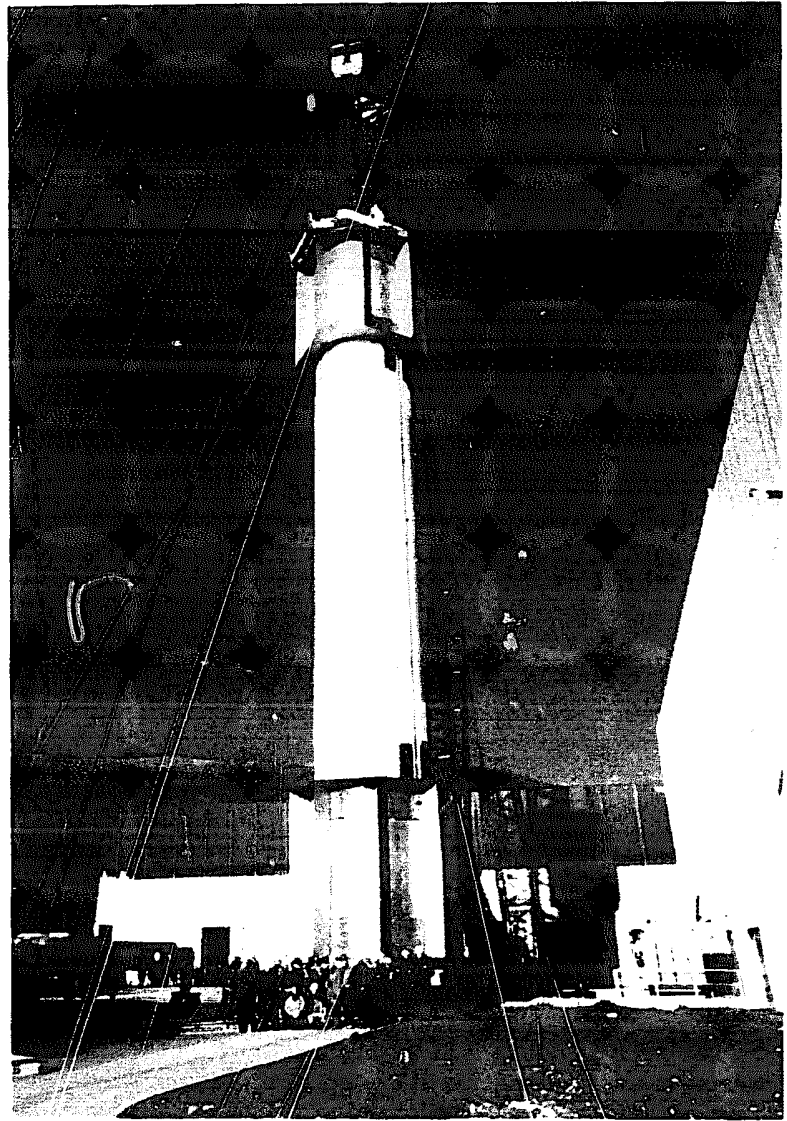

Fig. 27. The support tube being lifted by a giant crane. resolution at $100 \mathrm{GeV}$. This is achieved using a configuration of three layers of drift chambers which very precisely measure the curvature of the muon trajectory in the region between the support tube and the magnet coil. In this region, the $0.5-\mathrm{T}$ magnetic field makes a 50 $\mathrm{GeV}$ muon track deviate from a straight line by a sagitta:

\section{$s=3.4 \mathrm{~mm}$.}

To get $\Delta m / m=(1 / \sqrt{2})(\Delta p / p)=1.4 \%$, we must measure $\Delta s / s$ to $2 \%$, i.e. $\Delta s \approx 70 \mu \mathrm{m}$. Very good mass resolution is required for the missing mass Higgs search according to the reaction:

$\mathrm{e}^{+} \mathrm{e}^{-} \rightarrow \mathrm{Z}^{0}+$ Higgs, $\mathrm{Z}^{0} \rightarrow \mu^{+} \mu^{-}$.

Fig. 32 shows a computer simulation of such an event in our detector.

The muon detector must be modular to fill the large volume of $1000 \mathrm{~m}^{3}$. It consists of two ferris wheels, each having eight independent units or octants (fig. 33). Air pads on the support tube allow rotation during the installation phase. Rails are used to roll the assembled ferris wheel inside the magnet. The octants are attached to the torque tube (fig. 34).

Each octant consists of a special mechanical structure supporting five precision chambers. There are two chambers (MO) in the outer layer, two chambers (MM) in the middle layer, and one inner (MI) chamber. They measure track coordinates in the bending plane. In addition, the top and bottom covers of the MI and MO

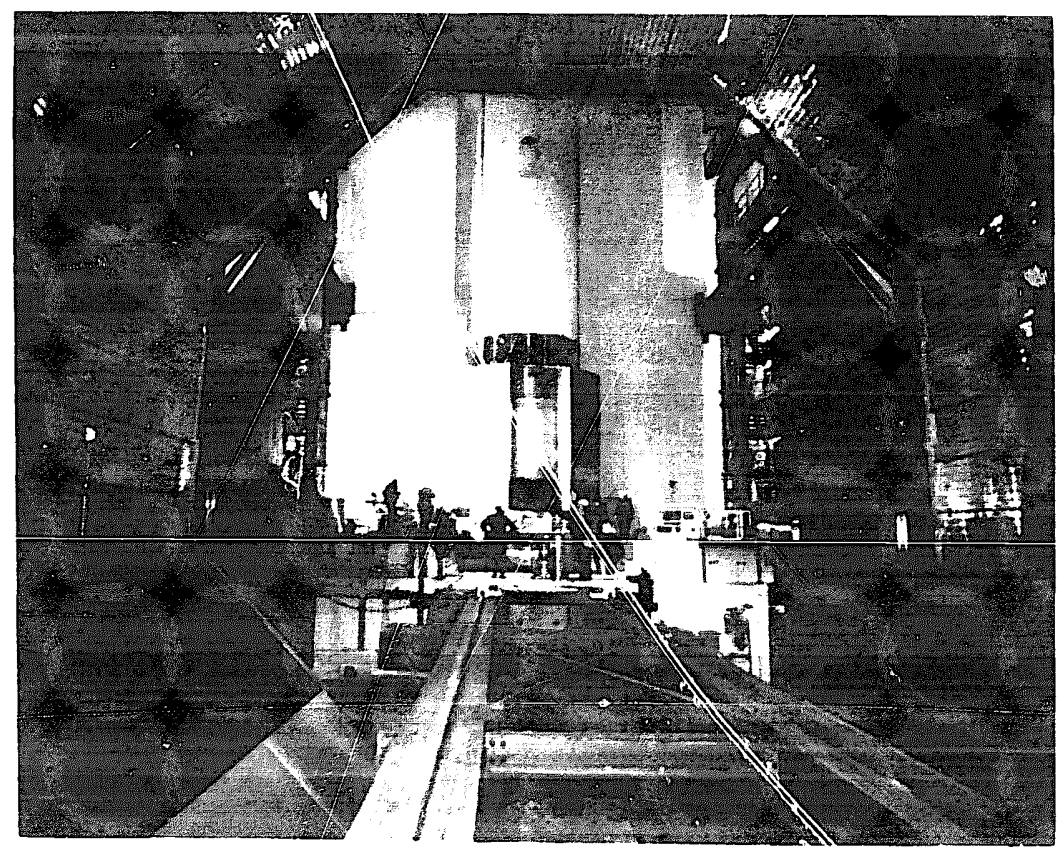

Fig. 28. The support tube arriving at the bottom of the acces shaft. 


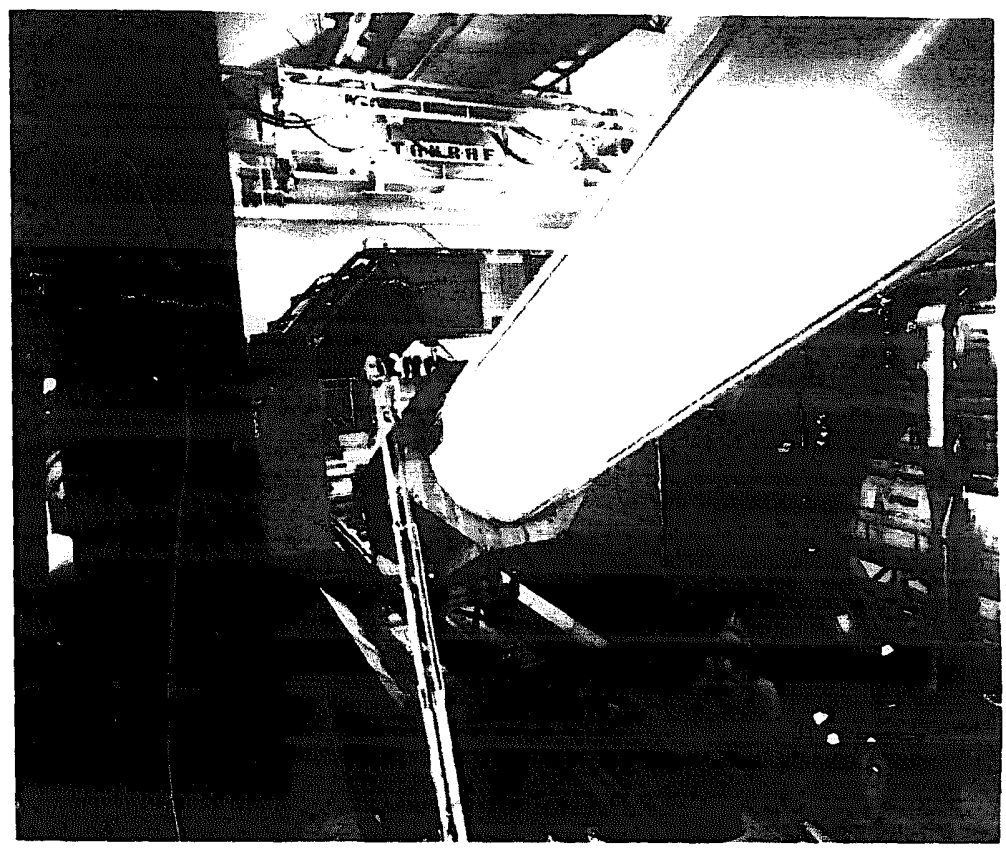

Fig. 29. The support tube entering the magnet.

chambers are also drift chambers and measure the $Z$ coordinate along the beam. There are a total of six $Z$ chambers per octant. Prime consideration was given to the accuracy of the sagitta determination. Our detector has been designed to minimize the contributions from the major causes of errors in the sagitta measurements which are:

a) intrinsic resolution of the drift chambers;

b) multiple scattering;

c) accuracy of alignment of chambers belonging to different layers.

An intrinsic accuracy of $250 \mu \mathrm{m}$ per wire is sufficient to reach the design resolution. Careful chamber optimization studies have led to smaller values [3]. We average

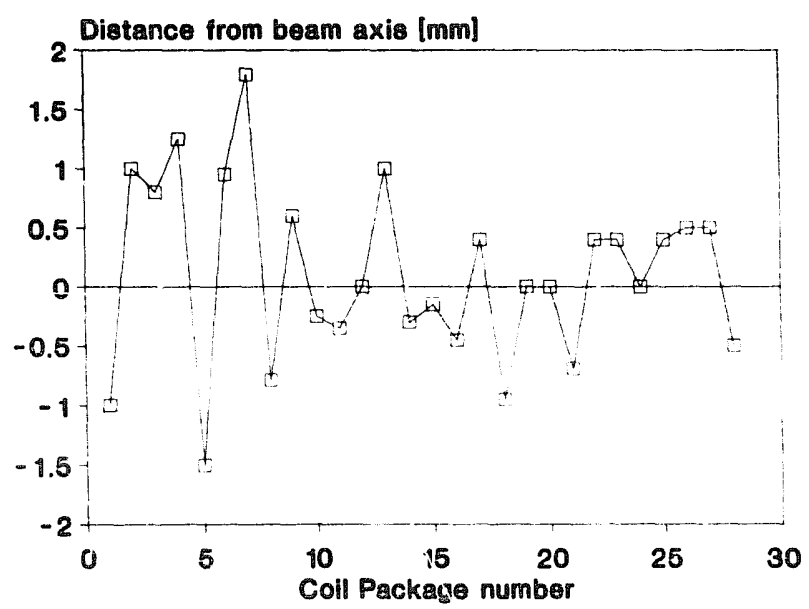

Fig. 30. Coil alignment. over several wires to obtain the final tracking accuracy. Multiple sampling improves the resolution by a factor $\sqrt{n}$ over the single wire resolution. MI, MM, and $\mathrm{MO}$ sample the muon track $n=16,24$ and 16 times, respectively. The resulting measurement error is:

$\Delta s=\left(\epsilon_{1}^{2} / 2+\epsilon_{2}^{2}\right)^{1 / 2}$.

with $\epsilon_{1}$ and $\epsilon_{2}$ defined in fig. 35 .

We use thin aluminum honeycomb with an average of $0.9 \%$ of a radiation length per two layers to enclose the middle chambers. Using this design, a multiple scattering induced sagitta error of $<30, \mathrm{~m}$ at $50 \mathrm{GeV}$ was reached.

Regarding pcint c) above, with these small sagitta values, muons more energetic than $3 \mathrm{GeV}$ will be con-

$X=0, Y=0,1=30338 A$

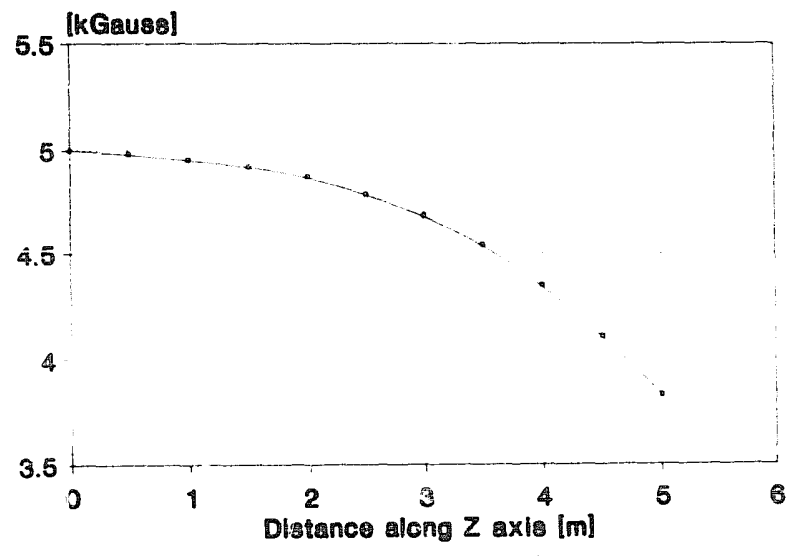

Fig. 31. Measured field. 


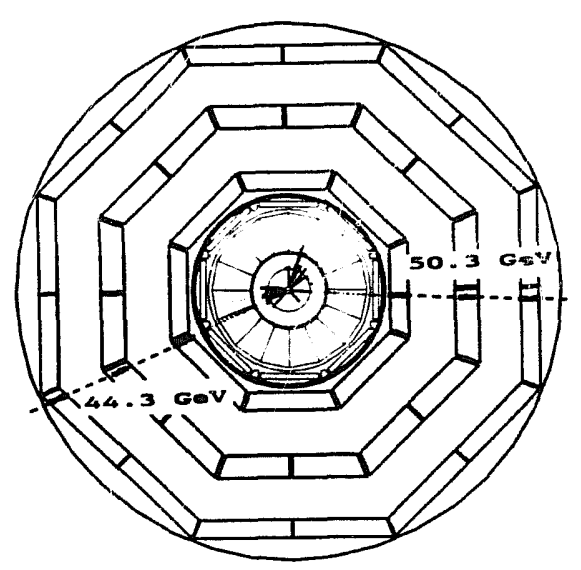

a)

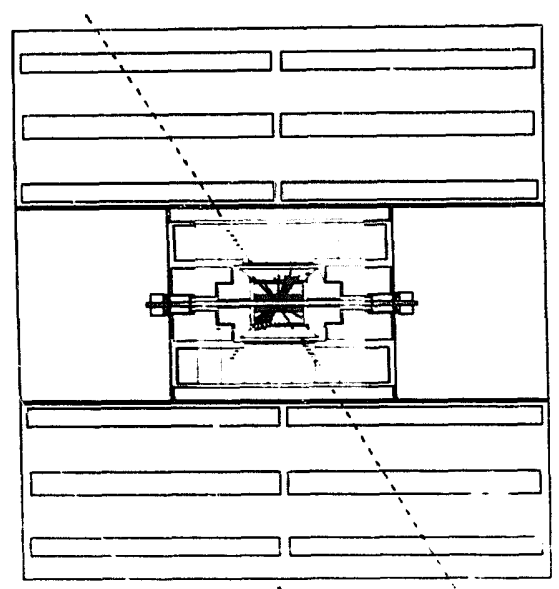

b)

Fig. 32. End view (a) and side view (b) of the L3 detector. Tracks in the muon chambers are those of a computer simulated event of the type $\mathrm{e}^{+} \mathrm{e}^{-} \rightarrow \mathrm{Z}^{0}+$ Higgs with $\mathrm{Z}^{0} \rightarrow \mu^{+} \mu^{-}$ and Higgs $\rightarrow b \bar{b}$ fragmentation.

fined to one octant. Therefore, alignment is only critical between chambers of the same octant (fig. 36). To achieve the design resolution, systematic errors in the internal octant alignment must be kept below $30 \mu \mathrm{m}$. As described later, this requires complex optical and

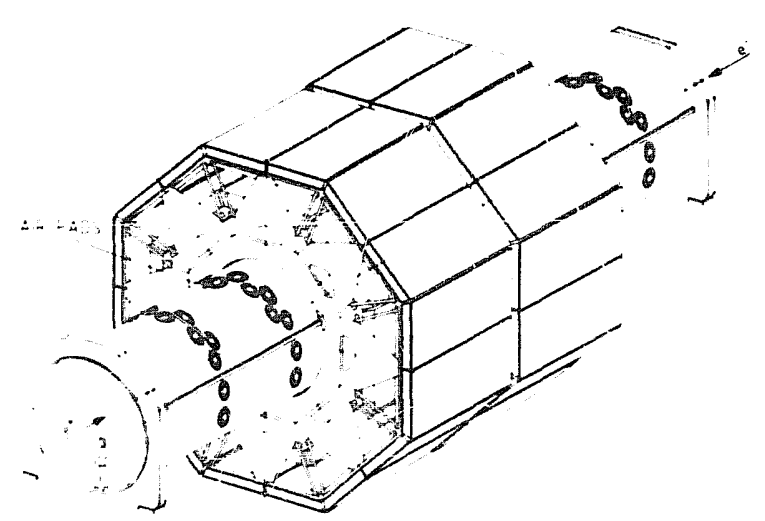

Fig. 33. Schematic view of the assembled muon detector.

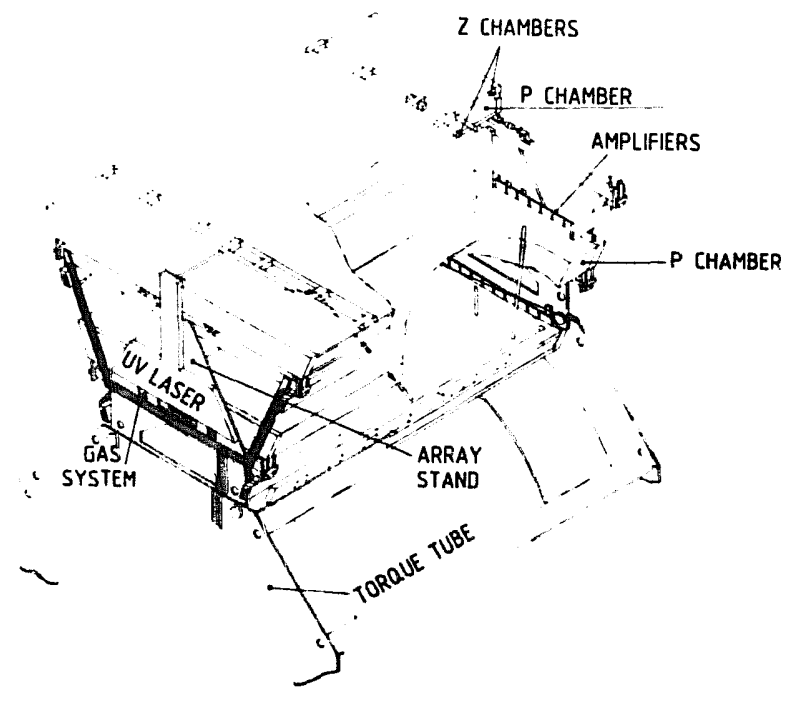

Fig. 34. An octant module is attached to the supporting torque tube. Precision chambers, $Z$ chambers, octant support stand, amplifiers and cables, gas system, and the UV laser calibration system are also shown.

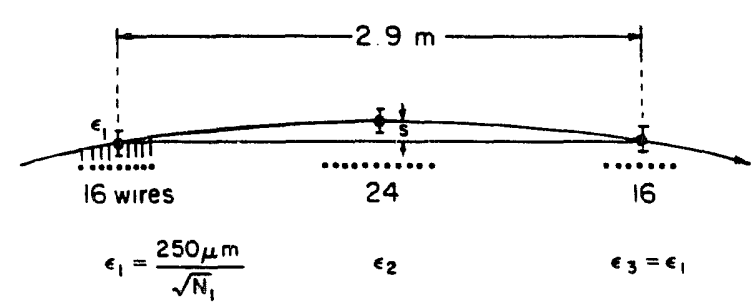

Fig. 35. Measurement errors on the sagitta of a muon trajectory curved in a magnetic field.

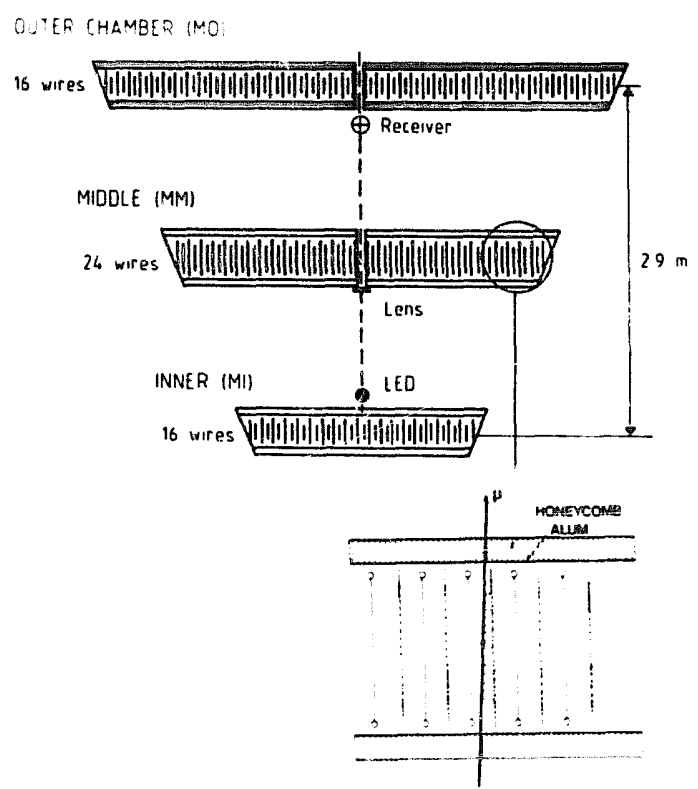

Fig. 36. End view of the three chamber layers in one octant, with a schematic view of the alignment system and a detail of the middle chamber. 


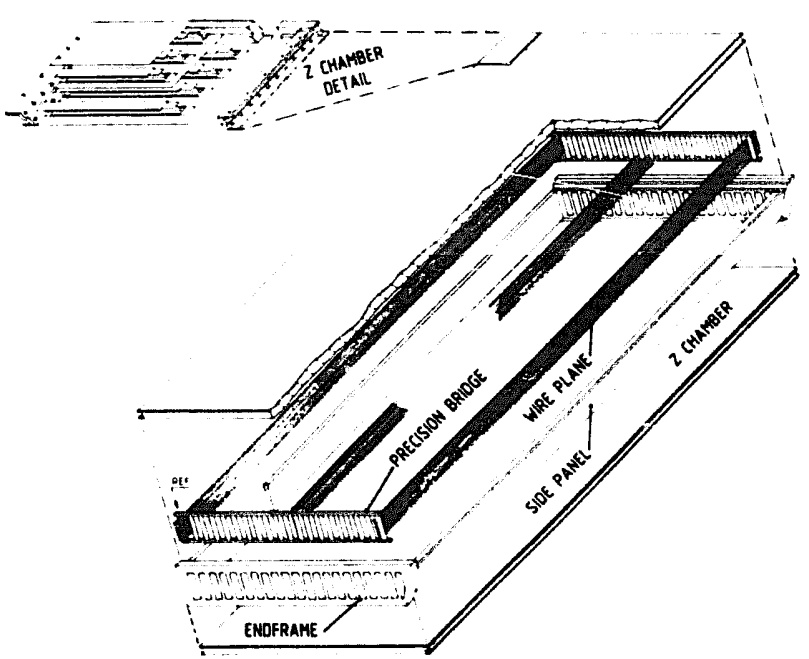

Fig. 37. Exploded view of an outer (MO) precision chamber and detail of $Z$ chamber.

mechanical measurements as well as UV laser and cosmic ray verification. Sagitta errors less than $30 \mu \mathrm{m}$ have been achieved in all octants.

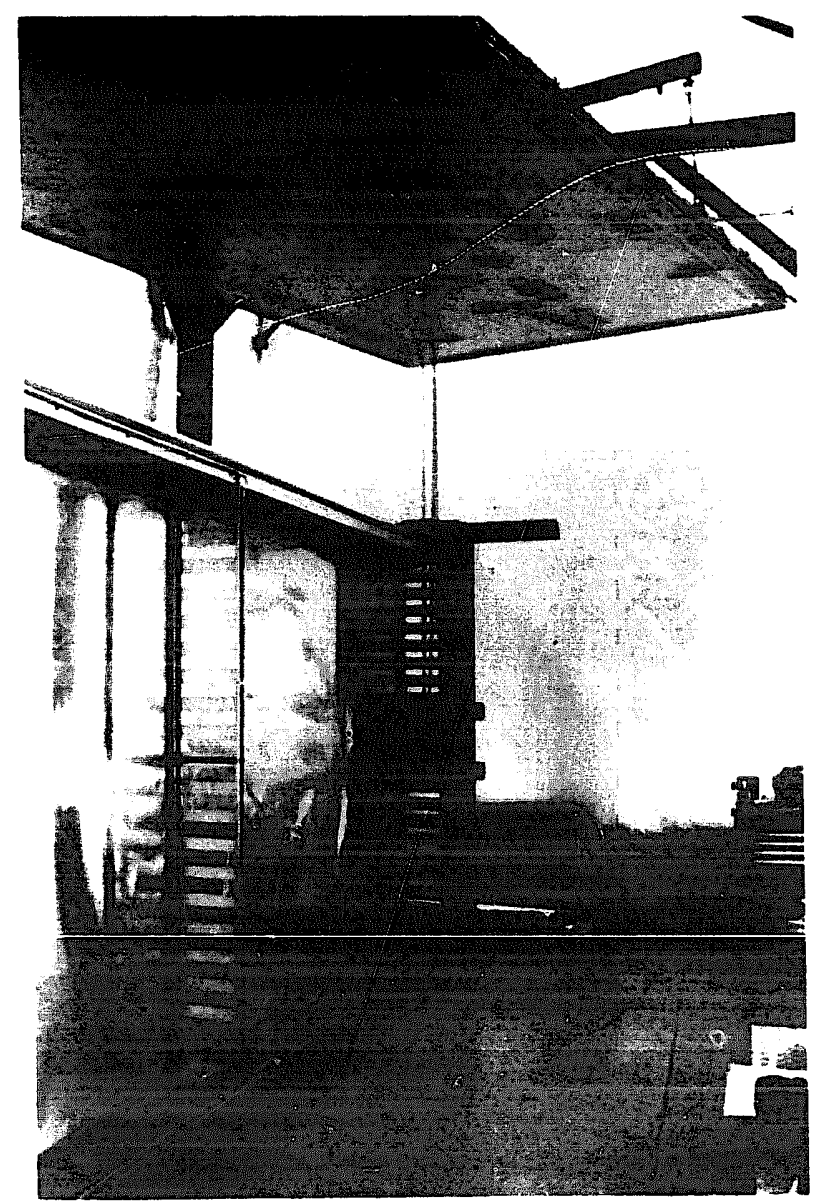

Fig. 38. An assembled chamber is shown before it is closed by a $Z$ layer.

\subsection{Precision chambers}

The momentum measuring, or " $P$ " chambers, are constructed of two cast and machined aluminum end frames, and two extruded aluminum side panels. The inner and outer chambers are closed on the top and bottom by $Z$ chambers. The middle chambers are closed by honeycomb panels to avoid degradation of the resolution due to multiple scattering. An exploded view of the mechanical structure of one MO (MI) chamber is shown in fig. 37, and fig. 38 shows a photograph of an assembled chamber just prior to $Z$ chamber mounting. There one can see the 5.6-m long wire planes positioned by "end bridges" inside the gas-tight box of end frames and side panels. The insert in fig. 37 depicts the double plane configuration of the $Z$ layers.

Each $\boldsymbol{P}$ chamber contains about 320 signal wires and a total of $\mathbf{3 0 0 0}$ wires. The signal and field shaping wires are positioned to about $10 \mu \mathrm{m}$ in the magnetic bending direction and to better than $40 \mu \mathrm{m}$ in the nonbending direction by precision Pyrex glass and carbon fiber bridges (fig. 39a). These bridges have very small thermal expansion coefficients $\left(1.5 \mathrm{ppm} /{ }^{\circ} \mathrm{C}\right)$ so that temperature effects on the wire positions are negligible. The
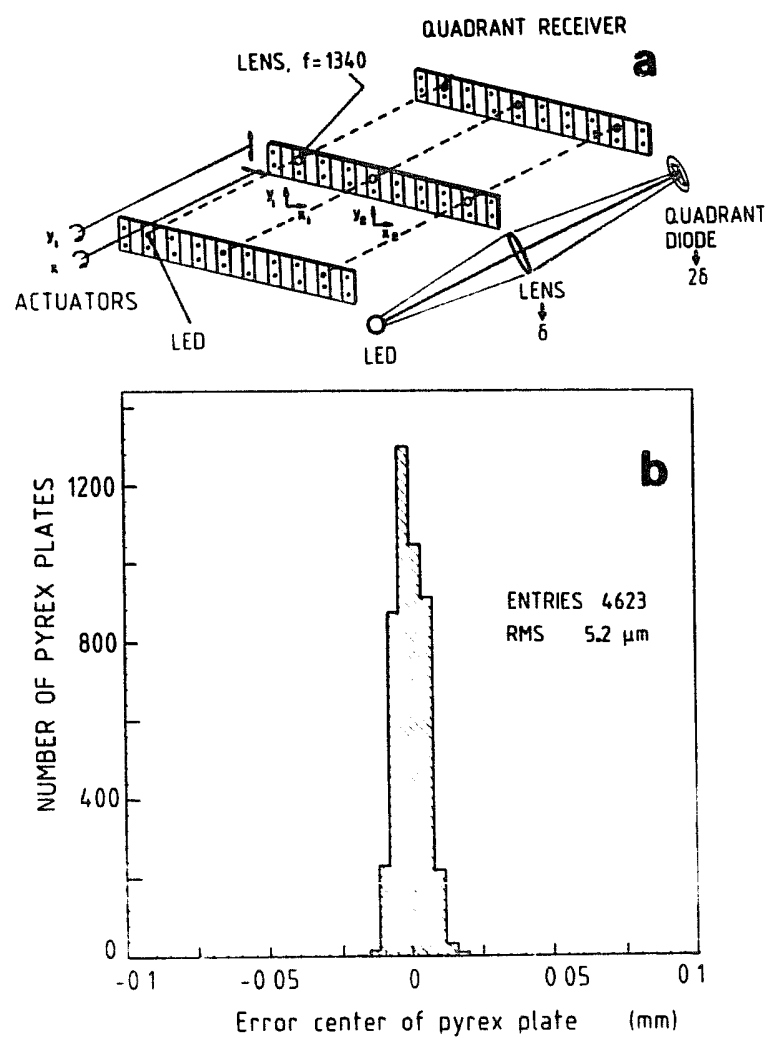

Fig. 39. (a) Three bridges support the wires. Three straightness monitors and actuators are schematically indicated. Added is a schematic showing the details of the straightness monitor. (b) The accuracy of the bridges can be seen from this distribution of errors in the equidistant locations of the Pyrex glass pieces. 
Pyrex glass pieces were glued to carbon fiber supporting bars using a very precise Invar template. Bridges thus produced have a surface-to-surface spacing of 101.500 mm. All bridges were measured using an HP laser interferometer system with an accuracy of $2 \mu \mathrm{m}$. The measured rms absolute position accuracy for all 255 bridges produced was $5.2 \mu \mathrm{m}$ (fig. 39b).

There are three bridges per chamber. One bridge at each end precisely positions the wires; the bridge in the middle reduces the sag of the wires by a factor of four (fig. 39a). The two end bridges are positioned with respect to external reference surfaces. The wire planes are put into position in the chamber and adjusted in length so that the vibrational frequency of the first

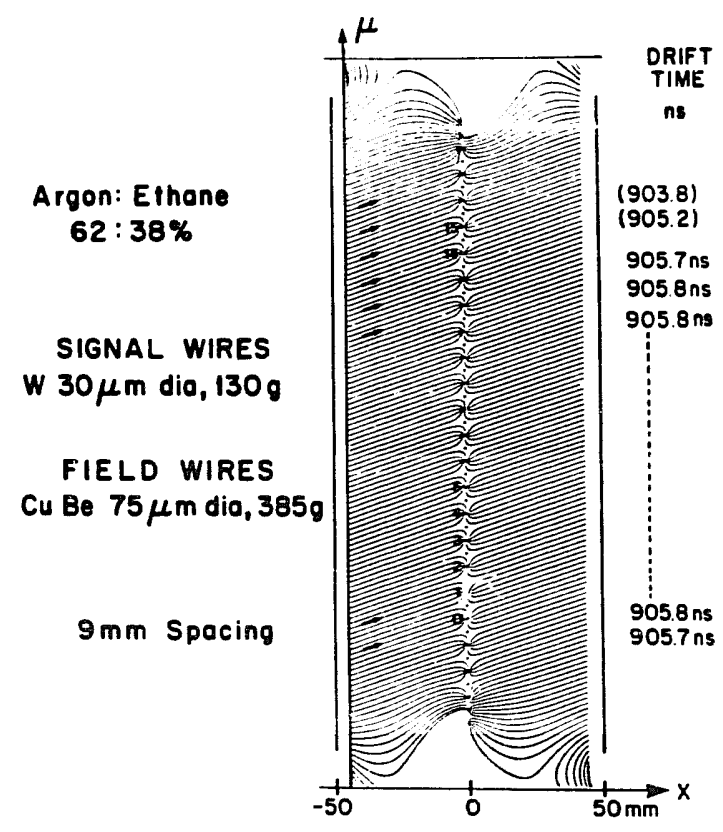

a)

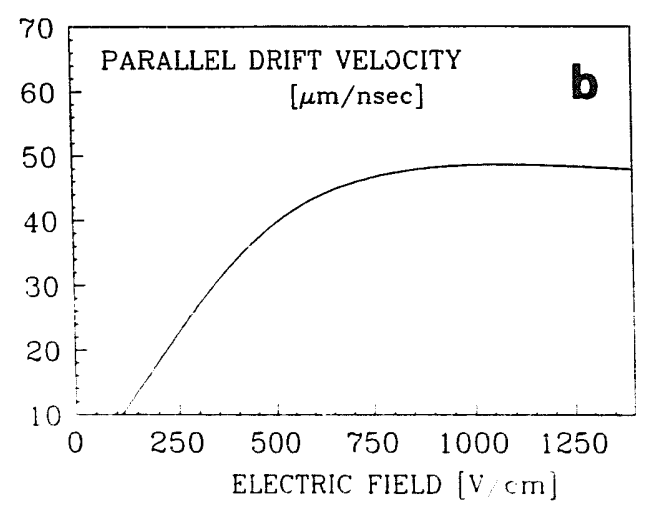

Fig. 40. (a) Electric field lines in a drift cell of an inner or outer chamber are shown with a 0.5-T magnetic field parallel to the wires. Drift times refer to a track 44-mm from the signal wires.

(b) Drift velocity computed as a function of the drift field.

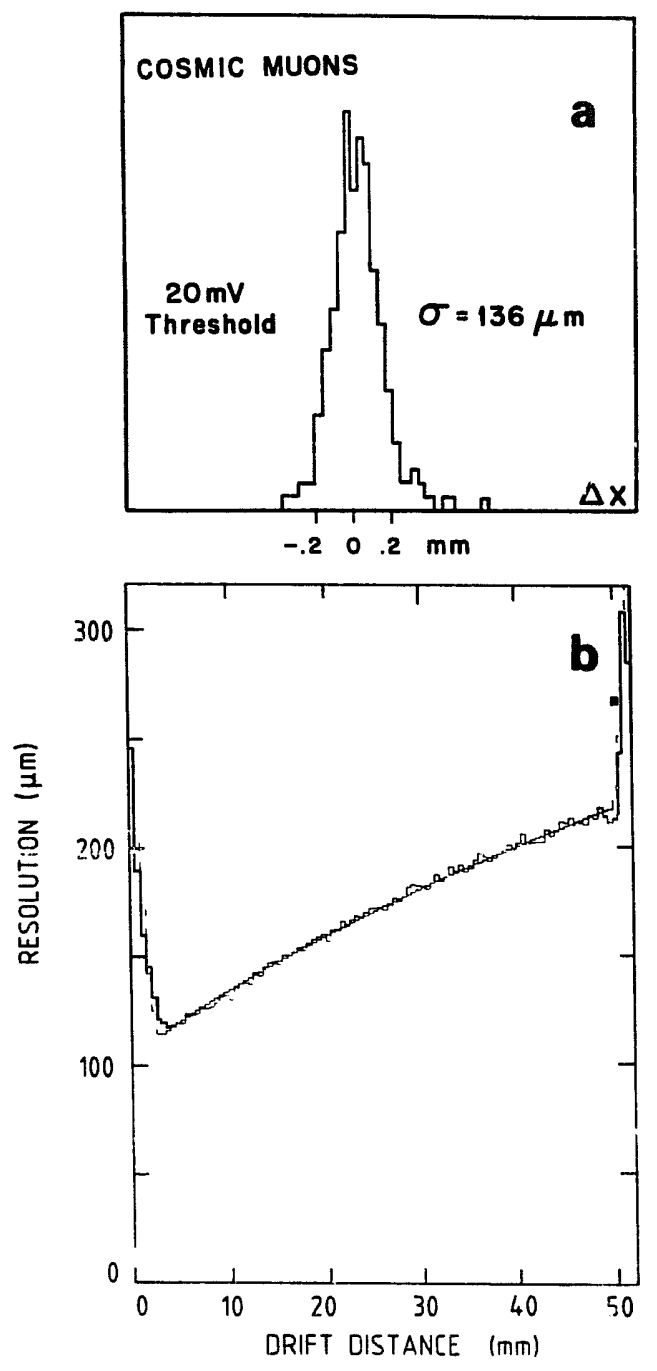

Fig. 41. (a) Distribution of residuals for cosmic muon tracks fitted to 14 of 16 wire measurements. (b) Resolution as function of the drift distance measured in $0.5 \mathrm{~T}$ with a test chamber.

harmonic is $27.85 \pm 0.2 \mathrm{~Hz}$ for signal wires. This ensures equal gravitational sags of $95 \mu \mathrm{m}$ for all signal wires.

Three internal alignment systems are integrated into the structure of the bridges (fig. 39a). This threefold alignment system [4] consists of LED, lenses and quadrant photodiodes. Light from an LED mounted on one end bridge is focused by the lens in the middle bridge onto the quadrant photodiode at the opposite end bridge. A displacement $\delta$ of the middle bridge moves the image by $2 \delta$ on the quadrant diode. The imbalance of the photodiode output measures this displacement. Each set has been individually calibrated on an optical bench and the systems are linear over a range of $\pm 250 \mu \mathrm{m}$. The middle bridge is moved by actuators 
in $x$ and $y$ (perpendicular to the beam direction) in response to computer readout of the system. The bridges are aligned when all four quadrants of the photodiode receive equal amounts of light. These systems allow us to position the bridges and thereby the wires to an accuracy of $10 \mu \mathrm{m}$. Deviations from zero occurring as functions of time or temperature are continuously recorded.

Wires are put into position along the Pyrex surfaces of the three bridges using a template. They are then attached to the Pyrex surfaces with an insulating wax. Since the Pyrex edges are optically flat, the average of the 16 (or 24) signal wires has little systematic error and one can determine the local track slope to 1-2 mrad. Fig. 40a depicts a chamber cell with 16 signal wires showing the computed drift paths in a 0.5-T magnetic field.

The chamber cell has been designed to have a very uniform electric field throughout the active region. Sense wires are spaced $9 \mathrm{~mm}$ apart and are interspersed with field wires. Eight additional wires beyond the last sense wire equalize the drift time behavior of all the sense wires within $0.2 \mathrm{~ns}(10 \mu \mathrm{m})$. A plane of cathode wires, spaced $2.25 \mathrm{~mm}$ apart, is at $50.75 \mathrm{~mm}$ from the sense wire plane. Four different high voltages are applied to the sense, field, cathode and guard wires, allowing us to control the drift field, the gas amplification and the zero potential position. At nominal voltage settings, in d $0.5-\mathrm{T}$ magnetic field and at $740-\mathrm{mm} \mathrm{Hg}$ pressure, the gas gain is $5 \times 10^{4}$. With an electric field of $1140 \mathrm{~V} / \mathrm{cm}$ in the drift region, the drift angle due to the Lorentz force is $18.8^{\circ}$.

Fig. $40 \mathrm{~b}$ shows the computed drift velocity for constant pressure, temperature and magnetic field. More precisely, the time-to-distance conversion function $x(t)$ $=v(\Phi, B, P) \times t$ in the drift cell has been mapped in test beam runs and its dependence on the track slope $\Phi$, magnetic field $B$ and barometric pressure $P$ was measured. Corrections are at most a few hundred $\mu \mathrm{m}$ near the sense and cathode planes. Without magnetic field, the measured cosmic ray residuals of a chamber with a threshold equivalent to the 10-12th drifting electron reaching the wire and two hits dropped is $136 \mu \mathrm{m}$ (fig. $41 a^{\prime}$.

The chamber resolution in a magnetic field depends on the distance from the wire plane and on the slope of the track. Resolution across the cell varies from $110 \mu \mathrm{m}$ to a maximum of $250 \mu \mathrm{m}$ close to the sense wire. Fig. $41 \mathrm{~b}$ shows the rms resolution as a function of the distance from the sense wire. The region of degraded resolution near the cathode plane is due to sloped tracks which are largely in the adjacent cell and to inhomogeneity in the electric field. From the data, we calculate that the overall $\mathrm{rms}$ chamber resolution is $168 \mu \mathrm{m}$. This is an average over all positions and slopes we expect for high momentum tracks.

\subsection{Z Chambers}

$Z$ chambers [5] consist of two layers of drift cells offset by one half cell with respect to each other to resolve left-right ambiguities. Each cell has two parallel aluminum I beams (fig. 37) connected to $-2.4 \mathrm{kV}$ and one gold-plated molybdenum anode wire with $50 \mu \mathrm{m}$ diameter at $+2.15 \mathrm{kV}$ in the center. The cell is closed by two aluminum sheets at ground potential and isolated from the I beam profiles by fiber glass strips.

The $Z$ chamber gas mixture $(91.5 \%$ argon and $8.5 \%$ methane) was chosen because it is not explosive. The drift velocity averaged over the cell is about $30 \mu \mathrm{m} / \mathrm{ns}$. The measured resolution, both in a test beam with a frototype and with cosmic rays in production chambers, is typically $500 \mu \mathrm{m}$. Since these chambers are the covering elements of inner and outer precision chambers, tight dimensional tolerances were needed to ensure mechanical fitting and thus precise machining was required on the $Z$ chamber frames. Relative wire spacing also depends on these frames. Aside from that, the design and technical specifications allowed for a rather simple construction. All of the $96 \mathrm{Z}$ chambers (dimensions about $6 \mathrm{~m} \times 2 \mathrm{~m}$ ) were built in two years with a production rate reaching six chambers per month at the end of the second year.

\subsection{Octant stands}

Octant stands are precision structures supporting the chambers and maintaining long term chamber alignment to $\leq 30 \mu \mathrm{m}$. The structures have been designed [6] to avoid tensor force transmission, thus octant behavior is fully predictable under all conditions of stress, load and temperature [7].

The main elements of the octant support structure are the A frames (fig. 42). They support each $P$ chamber at four points, two in each A frame. The MI chamber is mounted directly onto the $\mathbf{A}$ frame, while the other chambers are mounted on special support bars. A combination of support points with zero, one or two degrees of freedom ensures that chambers can move following temperature variations without introducing unwanted mechanical stresses. A longeron connecting the two A frames provides mechanical stiffness in the $Z$ direction.

In addition to the large structural components, there are approximately 300 small precision parts per octant. Materials were selected for strength, thermal characteristics and long term stability. Special materials, such as titanium and copper-beryllium have been used for chamber support feet, chamber tie-plates, torque tube joints and other highly stressed areas.

During the assembly phase, each octant undergoes detailed dimension and referencing checks; some of the design tolerances are listed in fig. $\mathbf{4 2 b}$. The four support 
feet on the floor which simulate torque tube attachment holes have been positioned in a plane to $50 \mu \mathrm{m}$. Critical distances between reference surfaces are measured to 5 $\mu \mathrm{m}$ by means of special tools calibrated with a laser interferometer. Angles are measured with electronic bubble levels [5] which have an intrinsic resolution of better than $1 \mu \mathrm{rad}$. A completed octant is shown in fig. 43.

After chamber loading, preliminary alignment and functionality tests, each octant is subject to a detailed alignment verification (see section 4.10). Then it is rotated to the angle corresponding to its final position in the ferris wheel and alignment is performed to the specifications of fig. $42 \mathrm{~b}$. The elastic nature of the structure is verified by the absence of hysteresis in octant rotation. This is important in view of the installation scheme on the torque tube. For these reasons, each octant is rotated through $360^{\circ}$, then its alignment is rechecked. All octants have been processed in this way and have shown full reproducibility, hence elastic behavior.

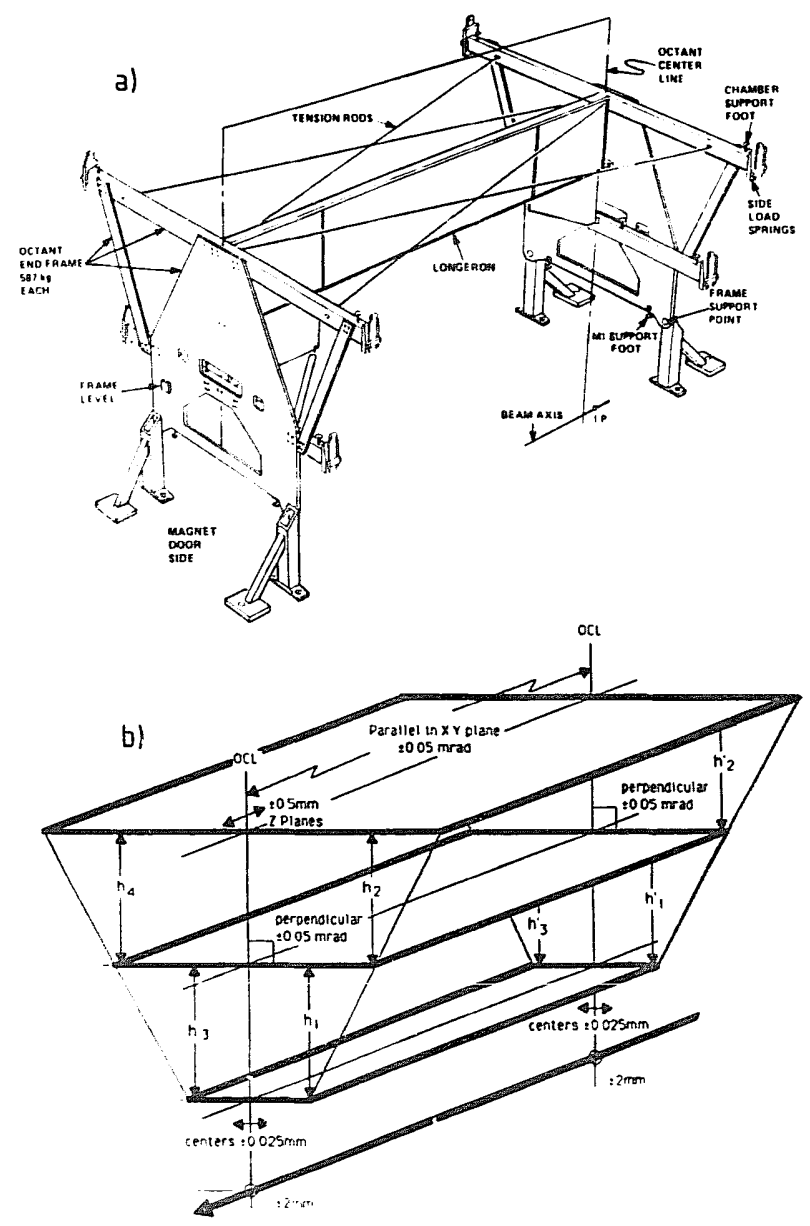

Fig. 42. (a) The octant stand structure. (b) Some of the alignment tolerances are shown for assembled octants. The alignment tolerance on the chamber center lines is only $25 \mu \mathrm{m}$

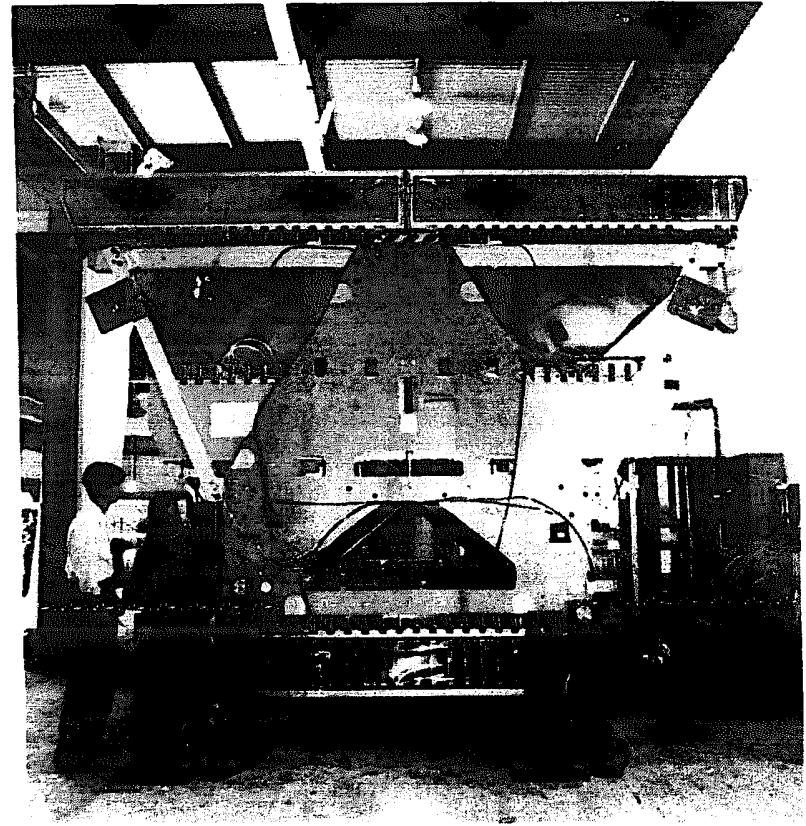

Fig. 43. End view of an octant.

\subsection{Opto-mechanical alignment: vertical alignment sys- tem}

The accuracy of the bridges inside the $P$ chambers and of the internal alignment systems built into them assures that wires within a chamber are precisely positioned with respect to each other. The next step is to relate the wires in one chamber to those in the rest of the octant.

Straightress monitors similar to that of the precision bridges are part of the octant alignment system [4] (fig. 44). A precision piece containing two LED is attached to each end frame of an inner chamber. An insulated brass pin referenced to the LED touches one wire of a signal plane. The end bridge can be moved so that the wire just makes or breaks its electrical contact with the pin. In this way, the end bridge positions are set to within a few $\mu \mathrm{m}$. The middle and outer chambers have a similar system of pins touching wires. These pin assemblies and thus the wire planes of opposing chambers are kept at the precise cell separations of 101.500 $\mathrm{mm}$ by gauge blocks. The assembly between middle chambers contains a lens and that between the outer chambers contains two quadrant diodes. Each end thus has two straightness monitors which were calibrated on an optical bench.

Based on the readout of these systems, the middle chamber can be moved on its titanium flexture feet to bring the chamber centers into a straight line with an error smaller than $10 \mu \mathrm{m}$. Readout of this system over a 4 -day period is shown in fig. $44 \mathrm{c}$. 

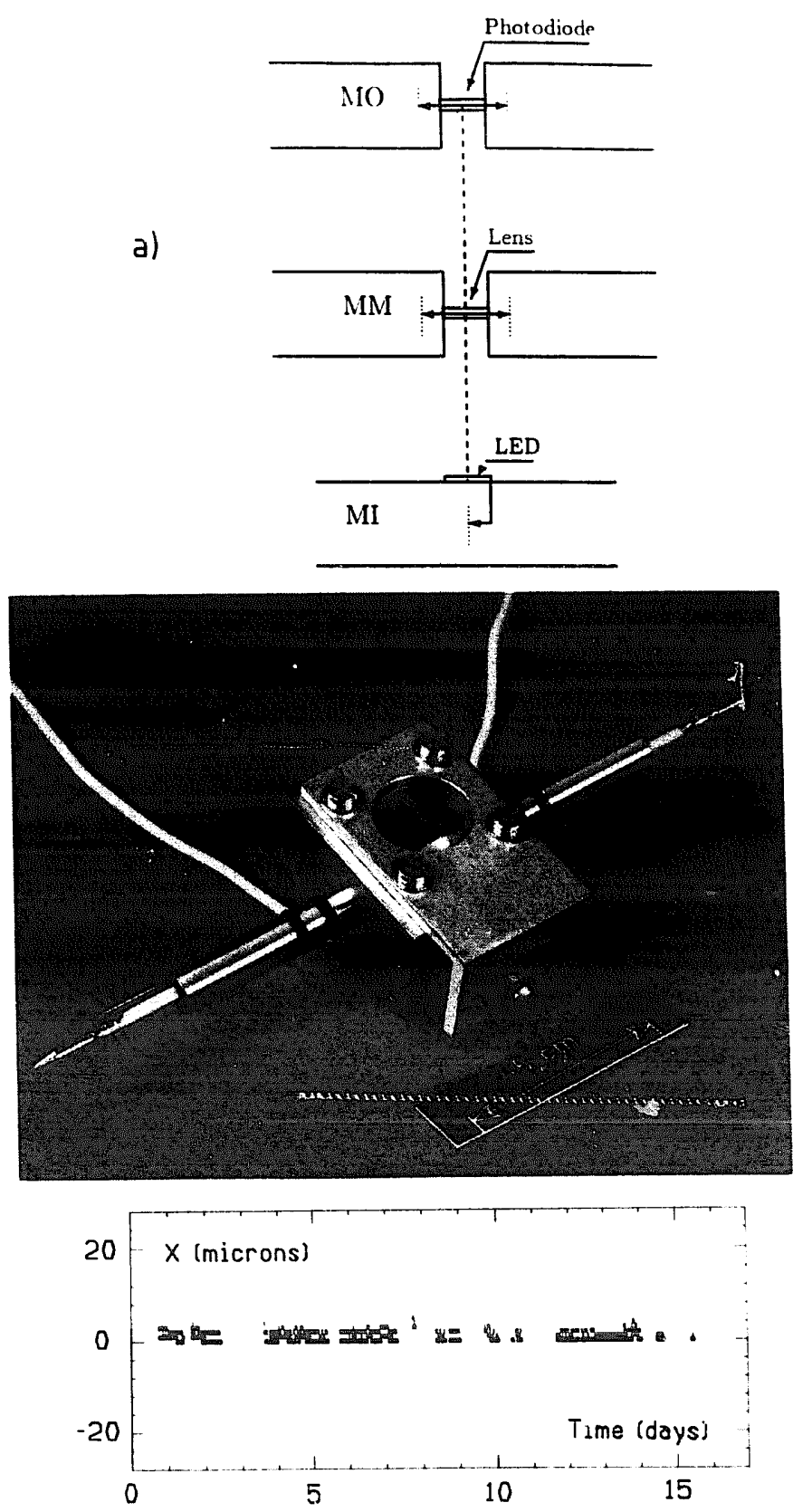

Fig. 44. (a) Principle of referencing the octant center line directly to the wires of the three chambers. (b) A vertical alignment piece assembly with a lens (for the middle chambers). (c) Relative position of the middle chamber in an octani monitored over a two weeks period.

\subsection{Laser beacon}

The vertical alignment systems guarantee that the chambers line up at each end of the octant, but the two octant center lines must also be parallel to each other. We use a laser beacon [9] to measure the degree to which the two ends of the octant are parallel. A He-Ne laser beam which is reflected by $90^{\circ}$ by a highly accurate rotating pentaprismatic mirror assembly sweeps out a plane to an accuracy of better than $30 \mu \mathrm{m}$. The deviation of the octant center lines from this reference plane is measured by six position sensors (multichannel photodiode arrays) attached directly to the three elements of each vertical straightness monitor. The laser beacon (fig. 45) can measure the angle between the two octant lines to better than $25 \mu \mathrm{rad}$, corresponding to an error in the sagitta of less than $10 \mu \mathrm{m}$. The $\mathrm{MO}$ and MM chambers are adjusted so that this measured angle is zero.

\subsection{UV laser}

Each of the 16 octants contains a two-stage nitrogen ultraviolet laser $[10,11]$, which is operated under computer control. The laser beam is directed up and across the top of the outer chamber layer by an addressable movable beam directional element (fig. 46). Mirrors direct the beam down through a quartz window into selected drift cells of all layers of the octant which are connected by tubes pointing roughly to the interaction point. Photodiodes at the bottom of the MI chamber measure the intensity and position of the beam centroid. Each octant has eight laser beam trajectories, which simulate infinite momentum particles coming from the interaction point. The sagitta of laser events should be zero $[10,11]$, and thus is used to verify the alignment. Two of the laser beams have movable mirrors and can produce parallel trajectories of exactly known separation, allowing us to measure and constantly monitor the electron drift velocity.

\subsection{Electronics}

The signals from $27648 P$ chamber wires are connected via $82-\Omega$ decoupling resistors to 13824 amplifiers [12] in corresponding pairs of wires from both detector wheels. Amplifiers are located in the median plane of the detector. The amplifiers convert incoming currents to voltages with a conversion factor of $25 \mathrm{mV} / \mu \mathrm{A}$. The differential output (typically $200 \mathrm{mV}$ for a muon) is sent via $30 \mathrm{~m}$ of twisted pair cable to discriminators [13] set to a $20-\mathrm{mV}$ threshold. The logical "time over threshold" signal is conducted through about $14 \mathrm{~m}$ of twisted pair cable to $500 \mathrm{MHz}$ FASTBUS time digitizers LeCroy LRS 1879 which continuously record until the common ston from the beam crossing arrives. The TDC cover a range of $1100 \mathrm{~ns}$ with $2.2 \mathrm{~ns}$ least bit accuracy.

The system has proved stable to $0.2 \mathrm{~ns}$ (corresponding to $10 \mu \mathrm{m}$ ) when checked by our standard $T_{0}$ calibration system [4] which electronically induces pulses onto the wires. All wires of the $Z$ chambers are similarly processed by 7680 time recording channels. Parallel outputs without time processing are used to form fast road triggers. 
a)

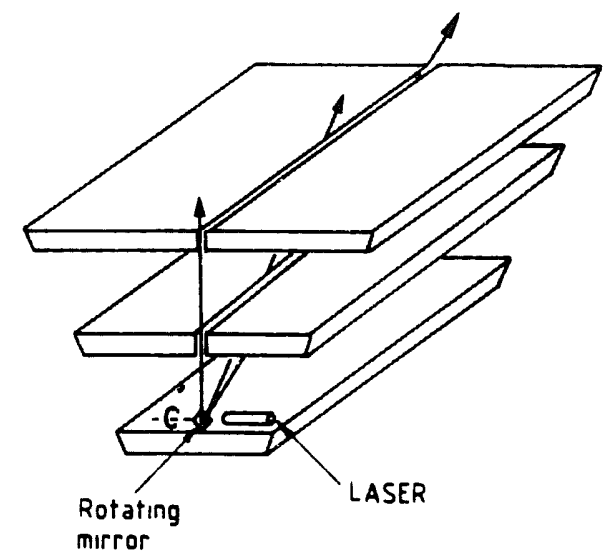

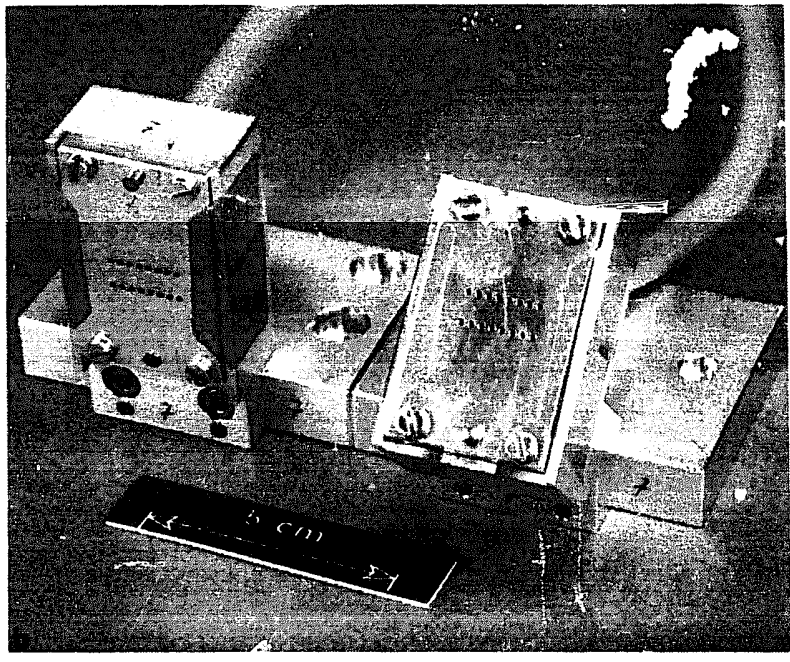

Fig. 45. (a) The laser beacon references six points in the center plane. (b) Two laser beacon sensors with precision templates for light transmission.

\subsection{Control and monitoring}

In addition to readout of the alignment systems, the laser beacon system and the UV laser system, there are other critical parameters which must be monitored and controlled:

- the chamber high voltage s $j$ stem,

- the signal pulse height,

- the time zero $\left(T_{0}\right)$ calibration,

- the octant temperature map,

- discriminator thresholds,

- preamplifier power supplies,

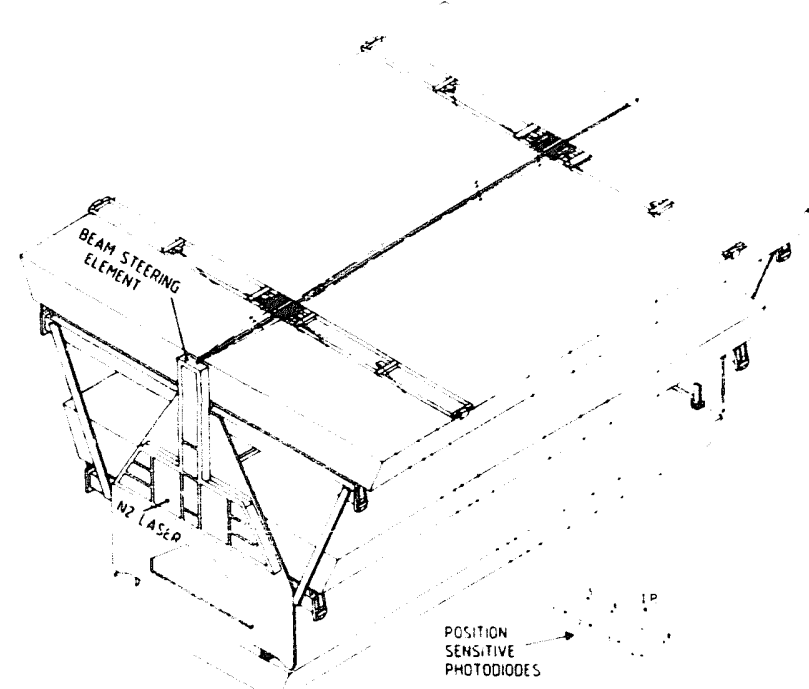

Fig. 46. UV laser alignment schematics: The beam from the nitrogen laser is directed by a beam directional element into eight trajectories. Position sensitive photodiodes which measure the location of the beam at the bottom of the inner chamber are schematically indicated.
- actuator motor position monitors,

- magnetic field measuring probes.

All of these tasks are performed by the monitoring system, which consists of a network of VME crates using an OS-9/68000 operating system. They have multitask, multiuser and stand alone capabilities, and also communicate with the main online computer.

\subsection{Results of alignment verification}

Each of the octants is adjusted as closely as possible to an ideal geometry using the double set of straightness sensors and the laser beacon. UV laser runs of 100 events are then taken using the eight different laser beam paths in each octant. Reconstruction of the laser trajectories (fig. 47a) should show straight lines. The deviation from zero called sagitta, which is a measure of our alignment accuracy, is defined as:

$s=-\left(x_{\mathrm{mi}}+x_{\mathrm{mo}}\right) / 2+x_{\mathrm{mm}}$,

where $x_{\mathrm{mi}}, x_{\mathrm{mm}}$ and $x_{\mathrm{mo}}$ are the coordinates measured in the inner, middle and outer chambers, respectively.

Fig. 47b shows measurements from 42 laser runs, for one octant. Errors on the points are obtained from the residuals of 100 event runs. The resulting average of all runs

\section{$-25 \pm 10 \mu \mathrm{m}$}

is within the design limit of $30 \mu \mathrm{m}$. The error is derived from the scatter of results from the different runs, thus taking systematic errors of the laser into account.

An independent way of checking octant alignment in the absence of a magnetic field uses cosmic muons. Scintillation counter hodoscopes are positioned above and below the octant, and moved to either end as desired. The resulting coincidence trigger is used as a TDC stop. Runs of about 10000 events are taken at 


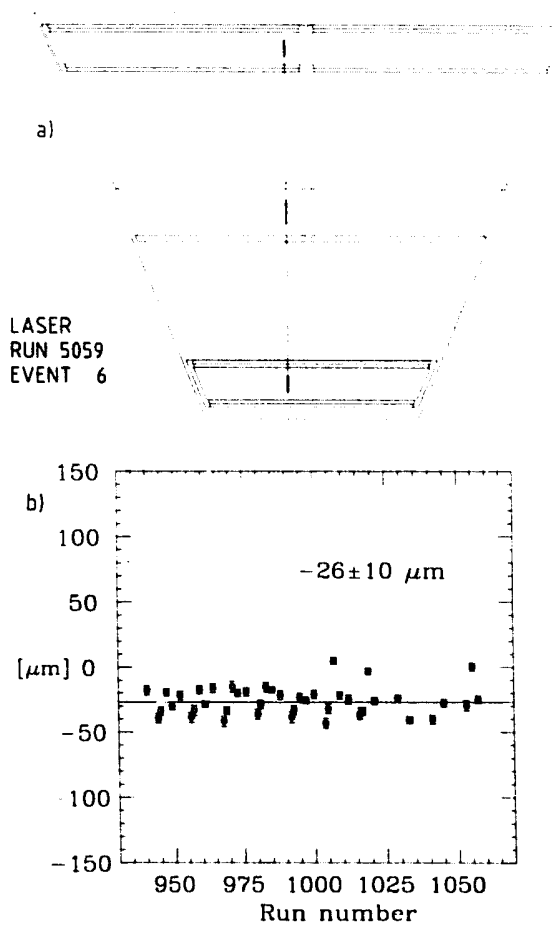

Fig. 47 (a) A computer reconstructed laser event. (b) Measured sagitta from a series of $\mathbf{4 2}$ runs of 100 laser shots each.

both ends of the octant. Tracks are reconstructed from the chamber segments as shown in fig. 48a.

Accepting all cosmic rays, we obtain the wide histogram in fig. $48 \mathrm{~b}$ which has an rms of $1.3 \mathrm{~mm}$. This large width is due to multiple scattering from the predominantly low energy cosmic muons.

The $\mathrm{L} 3$ muon chambers are unique in that they also measure the local slope of the particle trajectory to an accuracy of $\approx 1 \mathrm{mrad}$. Demanding that the local slope in a chamber agrees with the overall particle trajectory to within 2 mrad eliminates events with large multiple scatters. A narrow distribution of 760 events is obtained in fig. $48 \mathrm{~b}$. The centroid confirms that this octant is aligned to:

$s=(2 \pm 400 / \sqrt{760}) \mu \mathrm{m}=2 \pm 14.5 \mu \mathrm{m}$.

Fig. 49 summarizes the results for 16 octants. $s=0$ is given by the setting of the two independent opto-mechanical systems. The squares show verification of the geometry by UV lastr measurements. The cosmic ray data are shown in circles as a third independent measurement. We see exceilent agreement between all three methods, and find all octants to be well within the range of specification of $\pm 30 \mu \mathrm{m}$.

\subsection{Conclusion}

The $\mathrm{L} 3$ precision muon detector is unique in its conception and ability to detect dimuons with $1.4 \%$

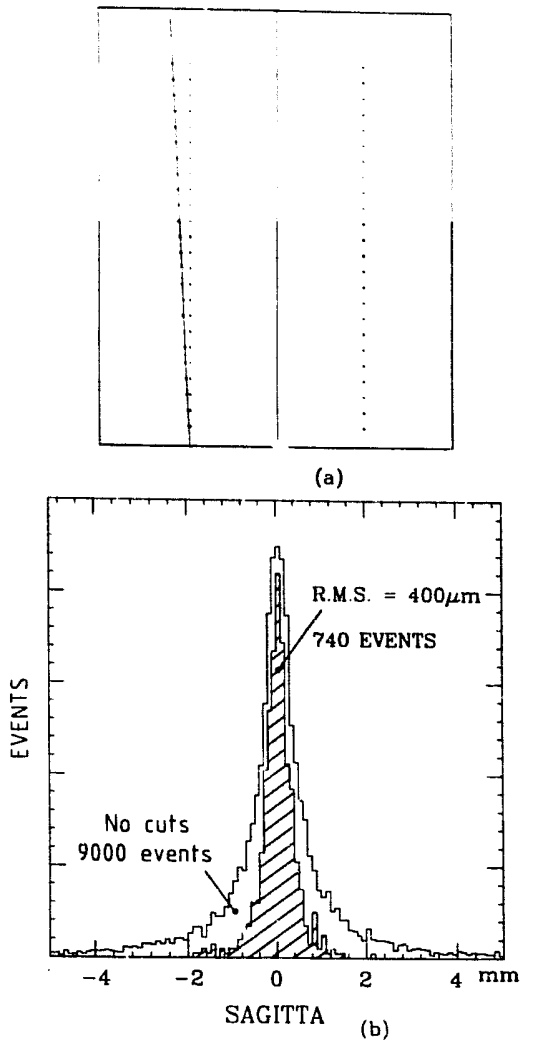

Fig. 48. (a) A cosmic ray track reconstructed in the middle chamber. (b) Sagitta distributions measured without cuts and with 2-mrad cut.

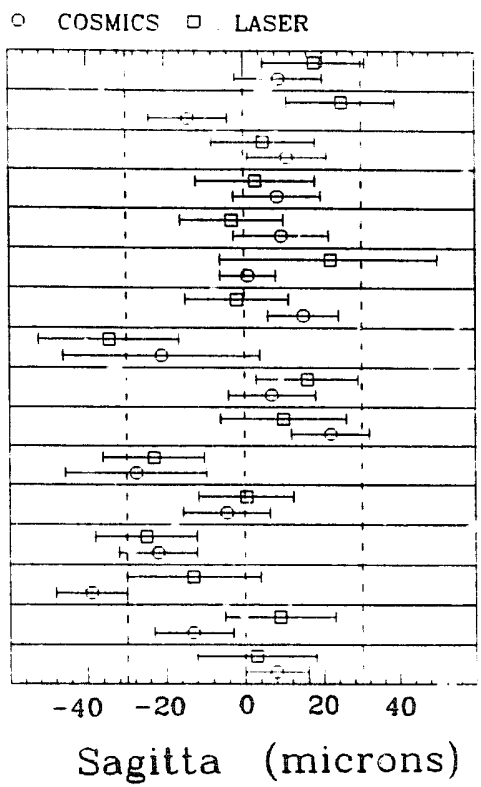

OCTANT 16

OCTANT 15

OCTANT 14

OCTANT 13

OCTANT 12

OCTANT 11

OCTANT 10

OCTANT 3

OCTANT 8

OCTANT $\tilde{\tau}$

OCTANT 6

OCTANT 5

OCTANT 4

OCTANT 3

OCTANT 2

OCTANT 1

Fig. 49. Compilation of alignment resuits for ail 16 octants. The zero sagitta prediction is the setting of the opto-mechanical system. UV laser verification results are shown as squares: circles indicate the center of cosmic ray distribution measurements. A $7-\mu \mathrm{m}$ systematic error on each laser measurement is not shown. 


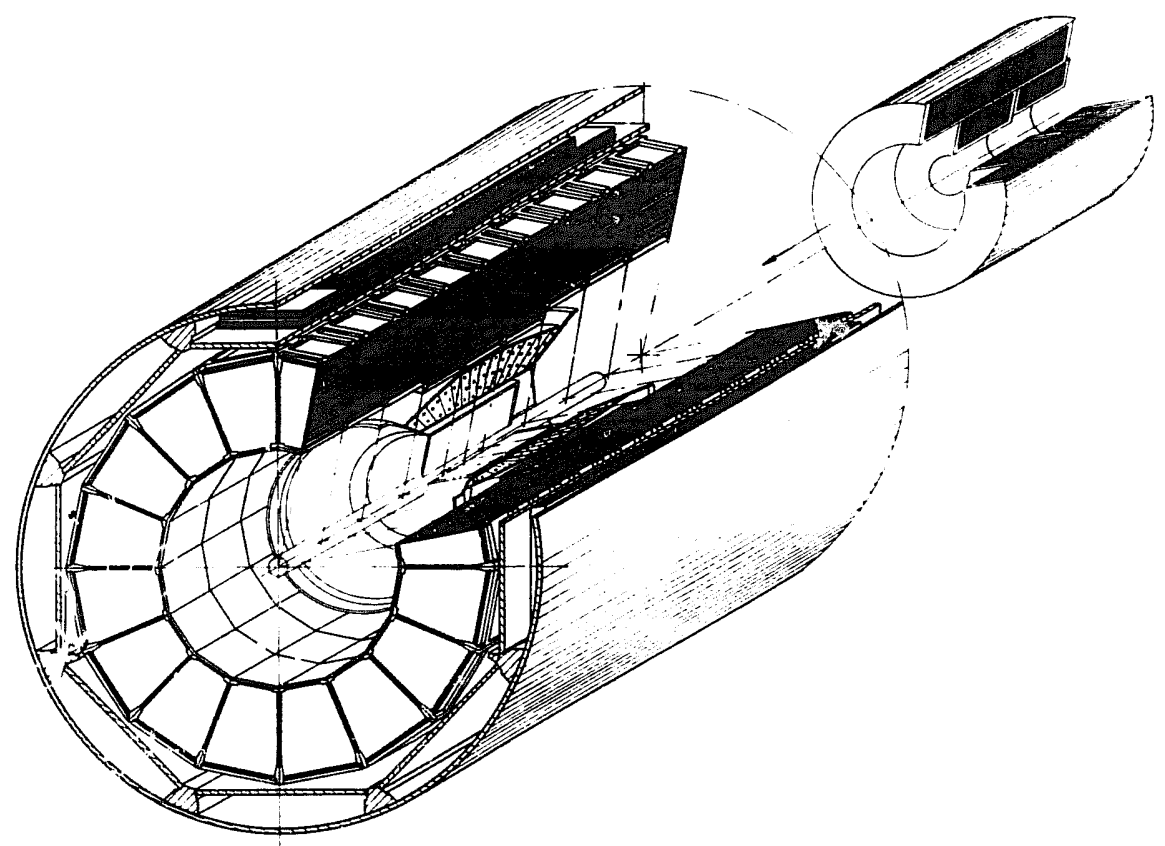

Fig. 50. Perspective view of the L3 hadron calorimeter.

mass resolution at the $Z_{0}$ mass. The critical feature, that of detecting (infinite momentum) straight tracks with $<3 n \mu \mathrm{m}$ sagitta error, has been demonstrated by three independent methods for all 16 productior modules.

This design can be extended to mea' ure muons at much higher energies.

\section{L3 barrel hadron calorimeter}

\subsection{Motivation and overall structure}

The energy of hadruis emerging from $\mathrm{e}^{+} \mathrm{e}^{-}$collisions is measured in $\mathrm{L} 3$ by the total absurption technique (calorimetry) with the BGO crystals and the uranium hadron calorimeter. The uranium hadron calorimeter has two parts: the barrel part and the forward-backward part. The hadron calorimeter barrel covers the central region $\left(35^{\circ}<\Theta<145^{\circ}\right)$; it is a fine sampling calorimeter made of depleted uranium absorber plates interspersed with proportional wire chambers; it acts as a filter as well as a calorimeter, allowing oniy nonshowering particles to reach the precision muon detector.

Uranium has a short absorption length, thus maximizing the amount of absorber material in the available radial space. The uranium radioactivity imposes stringent requirements on the construction and the operation $f$ the calorimeter, but it also offers a built-in gamma scurce for the calibration of the wire chambers [14]. We choose gas wire proportional chambers as detectors because they are stable, reliable, can operate in the magnetic field and are relatively easy to produce on a large scale. Moreover, in a multiwire detector the wires can be grouped in any readout pattern. By orienting the wires in alternate chamber planes at right angles to each other, better determination of particle trajectories is possible.

The barrel hadron calorimeter has a modular structure consisting of 9 rings of 16 modules each (figs. 50 and 51). The innermost ring is centered at the interaction vertex and is flanked on either side by one ring of long modules followed by three rings of short modules.

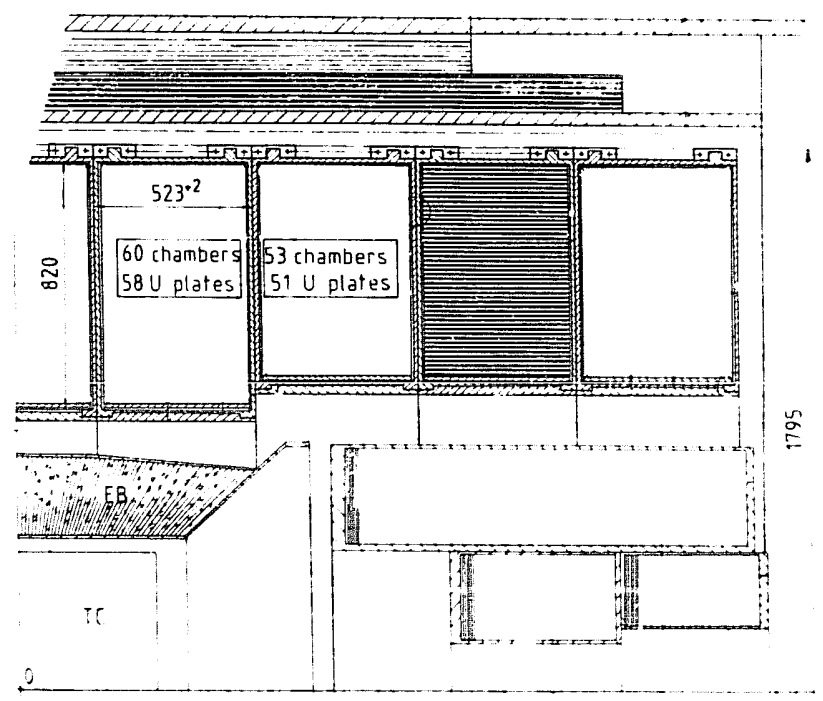

Fig. 51. Longitudinal cut of the hadron calorimeter. 
The hadron calorimeter barrel is $4725 \mathrm{~mm}$ long, has an outer radius of $1795 \mathrm{~mm}$ and an inner radius of $885 \mathrm{~mm}$ for the three inner rings and $979 \mathrm{~mm}$ for the outer rings. The 261-t assembled barrel was lowered to the experirinental area in one piece by the same giant crane used for the support tube.

\subsection{Proportional wire chambers}

The design of the chambers [15] is presented in fig. 52. The chamber gap is made as thin as possible without loss of mechanical stability for the anode wires at the working high voltage. Each chamber is made of a plane of brass tubes of equal length with $0.3-\mathrm{mm}$ thick walls and $5 \mathrm{~mm} \times 10 \mathrm{~mm}$ inner dimensions. The length of the tubes ranges from $347 \mathrm{~mm}$ to $605 \mathrm{~mm}$ depending on the position of the chamber inside the modules. The structural strength of the chamber body is assured by $0.7-\mathrm{mm}$ brass plates glued onto both sides of the chamber plane with self-adhesive Mylar sheets. The Mylar also insulates the tubes which are at a high potential during operation. The brass plates also shield the chamber from the uranium radioactivity, reducing the counting rate due to the uranium by an order of magnitude. The $50-\mu \mathrm{m}$ diameter gold-plated tungsten anode wires are crimped into gold-plated brass jacks, which in turn are
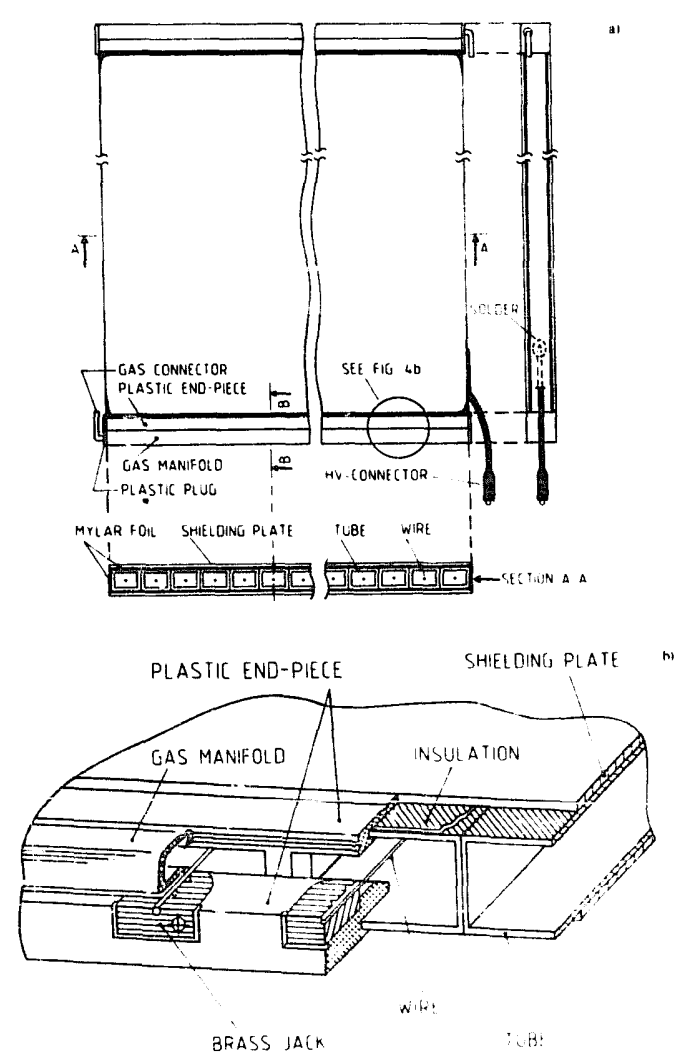

Fig. 52. Proportional chamber of the hadron calorimeter barrel.

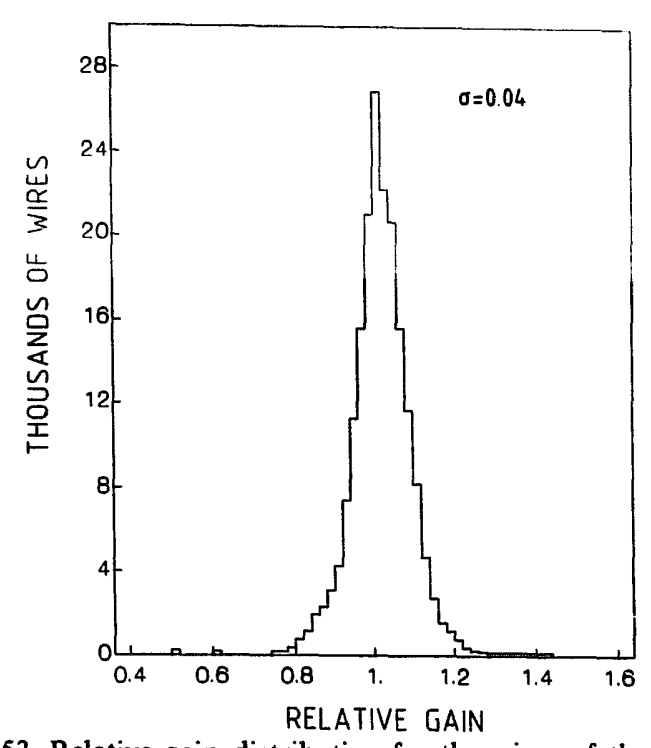

Fig. 53. Relative gain distribution for the wires of the chambers.

fitted into plastic end pieces. The tension in the wire is $250 \mathrm{~g}$ with an rms spread of $17 \mathrm{~g}$.

The gas is supplied in parallel to all tubes via two channels which are incorporated into the end pieces. The gas inlet and outlet are diagonally opposite to each other to ensure adequate flow of gas through all tubes. In designing the calorimeter particular attention was paid to minimizing the size of the dead regions such as the chamber end pieces, various support structures and space for the services. To achieve this, 53 different sizes of chambers are used, with the number of tubes per chamber ranging from 33 to 58 . For the same reason the chambers are operated with the anode wires at ground potential, avoiding the use of numerous capacitors.

All 371764 wires of 7968 chambers were tested in the production line [15] and showed good uniformity of response (fig. 53). This measurement was performed using both $\gamma$ rays from the natural radioactivity of uranium (fig. 54) and cosinic rays. Two independent tests were carried out to check the longevity of the chambers in a radioactive environment. In the first test the chambers were irradiated with a $10^{4}$ times more intense radioactive source over several days at the working high voltage. In the second test the chambers were operated in the normal uranium plate/chamber stack at 10 times higher proportional gas amplification (fig. 55). In both cases the chambers proved to be very stable and no aging effects were observed.

\subsection{Module design}

Both long and short modules have basically the same design. The inner part of the module resembles a tower mounted on a $15-\mathrm{mm}$ thick stainless steel base (fig. 56). 


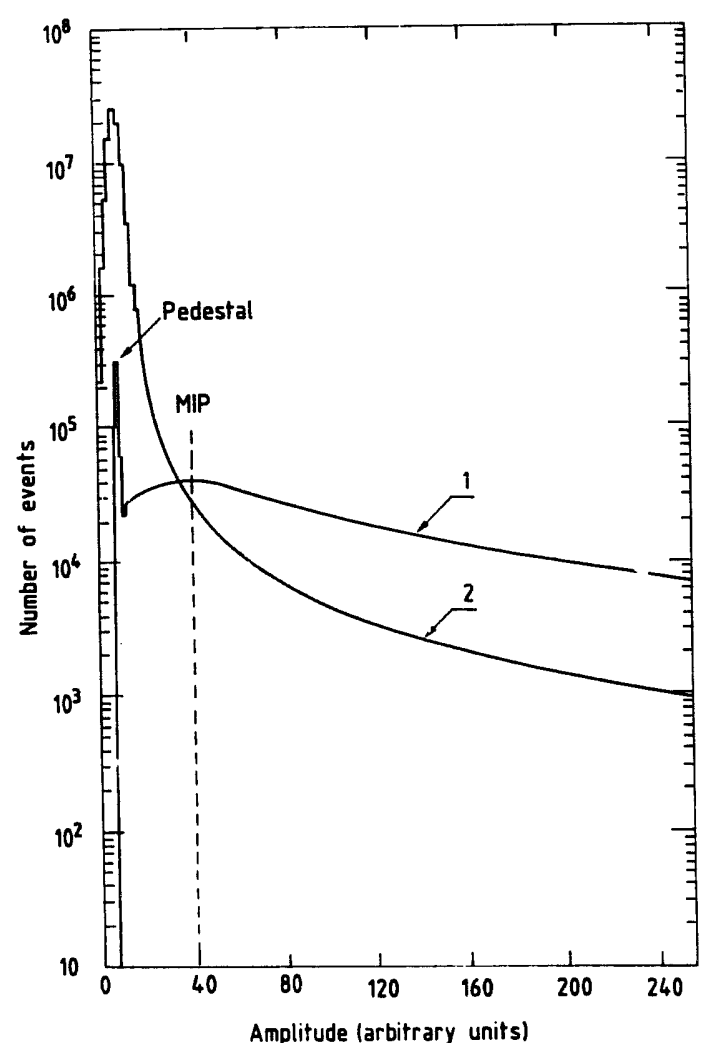

Fig. 54. Spectrum of signals caused by uranium radioactivity: (1) normal self trigger; (2) random gate tiigger.

The base and the top 15-mm thick stainless steel platc, connected with four spacer bars, constitute a supporting structure for the absorber/chamber stack. The spacers lie along the parallel faces of the modules. The 5-mm thick depleted uranium absorber plates are plated for safety reasons with approximately $20-\mu$ in thick copper-

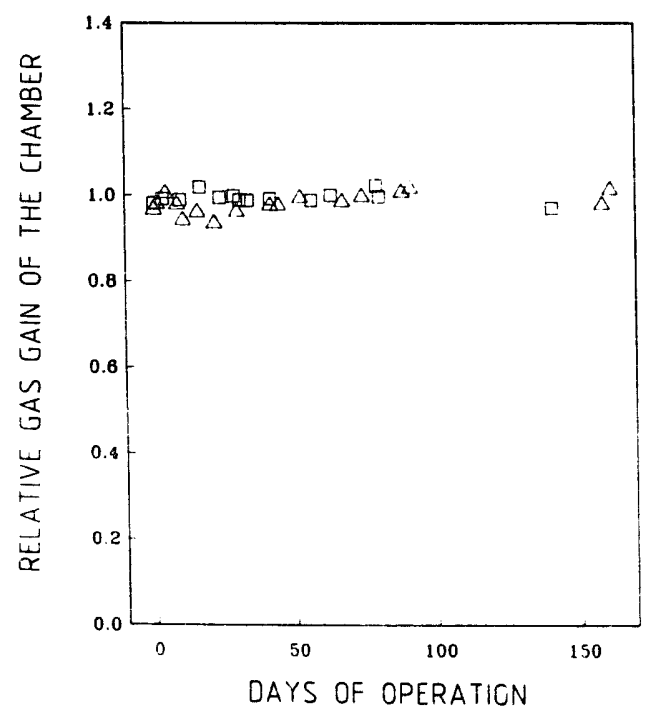

Fig. 55. Gain stability measurement.

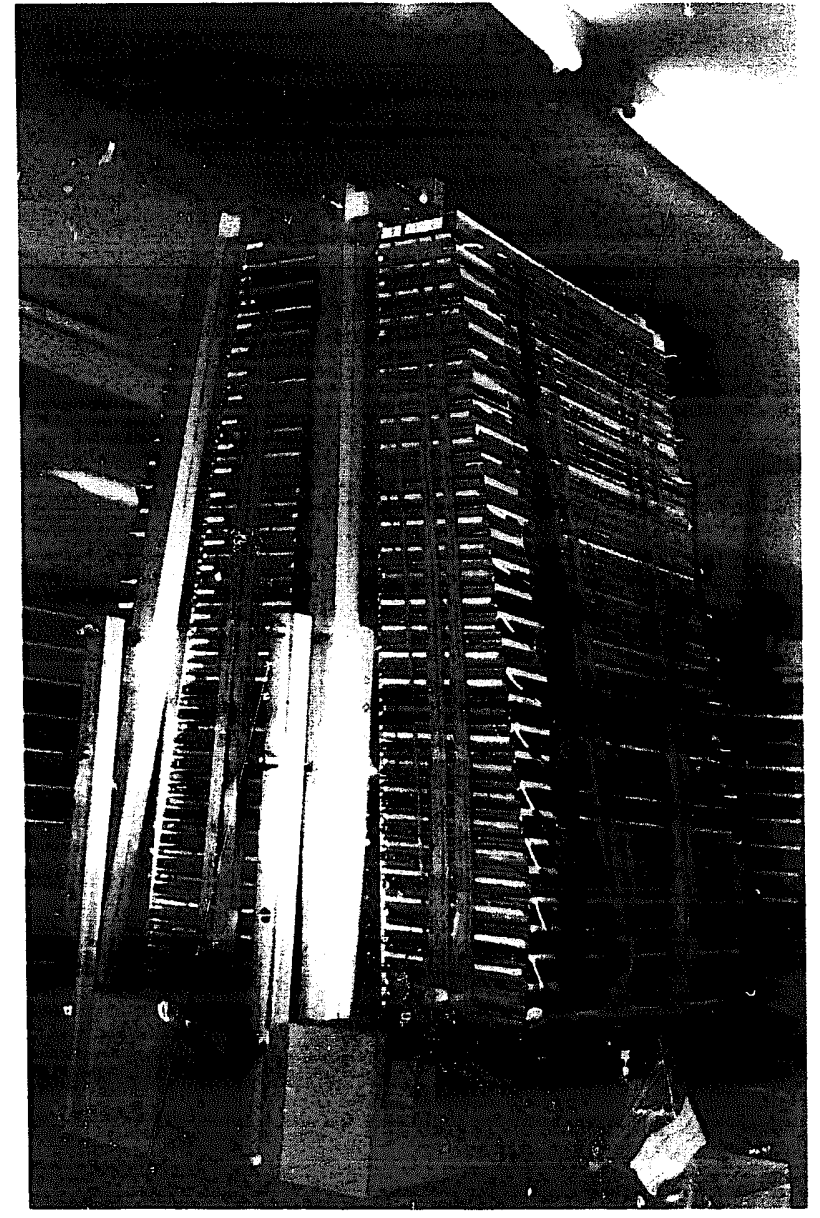

Fig. 56. Hadron calorimeter module.

nickel alloy. They are clamped into place in the supporting spacers. The chambers are put onto uranium plates and fixed with epoxy at four points. Long modules contain 60 planes of proportional chambers and 58 uranium plates plus the top stainless steel plate. This plate which lies between the BGO calorimeter and the uranium plates is part of the 54-mm shielding for the BGO photodiodes. Short modules contain 53 chambers and 51 plates of uranium plus the siainless steel platz. The dimensional tolerances of the absorber plates and the chambers are very tight, especially for the thickness. The chambers with wires parallel to the beam axis are referred to as $\Phi$ chambers and the ones with their wires normal to the beam are called $Z$ chambers. All services of the module are brought out through feedthroughs in the base plate.

The high voltage distribution system which is embedded in the base has four independent channels serving the odd and even numbered chambers in each projection. It contains one fuse per chamber, which can be blown in a controlled fashion. Thus if a chamber develops a serious problem it can be remotely disconnected without compromising the rest of the chambers 
in the group. The gas ( $80 \% \mathrm{Ar}+20 \% \mathrm{CO}_{2}$ mixture) is supplied serially to groups of five chambers in the same projection. Groups are connected in parallel to a common inlet and outlet located on the module base. The services are fitted into the space between the absorber/ chamber stack and the hood.

In four modules of each ring (top, bottom, left and right) the internal temperature is monitored at three positions with PT1000 sensors embedded into one of the spacer bars. The measurement accuracy is better than $0.5^{\circ} \mathrm{C}$. The information is used in the offline analysis to correct for the change in the gas gain due to temperature variations.

The hood is made of stainless steel plates welded along all edges. The slanting walls of the hood point to the beam axis and produce a gap in the acceptance. They, therefore, were made as thin as practical $(4 \mathrm{~mm})$. The parallel walls are $15 \mathrm{~mm}$ thick, while the top plate has a thickness of $22 \mathrm{~mm}$. The hood is bolted gas tight to the module base and locked to the $15-\mathrm{mm}$ top plate so that in any orientation of the module the space between the stack and the hood served for the services is preserved. The smallest chamber of the stack is situated between the hood and the $15-\mathrm{mm}$ plate. Gas fittings are provided in the base to permit the gas flow into and out of the space between the stack and the hood independent of chamber gas supply and return. In practice the exhaust from the chambers in each module is fed into the housing, maintaining a small over-pressure in the chambers. A long module weighs $1860 \mathrm{~kg}$, a short module $1720 \mathrm{~kg}$.

\subsection{Barrel mechanics and services}

The 16 modules are mounted on a $17-\mathrm{mm}$ thick stainless steel ring and are bolted together at their outer radii for added rigidity (fig. 57). Each assembled ring can move on two rails inside the support tube on two sets of rollers attached to the modules just below its mid-plane. The nine rings of the barrel are bolted together through the support plates of the rollers. In the assembled barrel (fig. 58) the 144 base plates form the outer surface of the barrel. The connections for the high voltage, preamplifiers, temperature sensors and gas piping are all situated on this surface. Preamplifier boards are plugged directly into the connectors in the base plate. The preamplifiers dissipate about $10 \mathrm{~W}$ per module and this heat is removed by liquid-cooled fins mounted on the base plate.

\subsection{Tower structure}

In order to measure the energy of hadrons and hadron jets, to separate two jets from each other and to determine the energy loss and the trajectory of muons passing through the calorimeter, a high degree of readout segmentation in the calorimeter is required. This need is accentuated by the fact that in some cases it is necessary to confine the measurement to the immediate region in which the energy is deposited in order to reduce the background from the natural radioactivity of uranium. To this end, the wires in each module are grouped to form readout towers (fig. 59). In the $\Phi$ projection the towers point to the beam axis with a constant angular interval. The segmentation is 9 in $\Phi$ and $Z$ for both kinds of modules and $10(8)$ in the radial direction for the long (short) modules. In the $Z$ projection the towers have a constant width, whereas in the $\Phi$ projection they point to the beam axis. The number of wires in each tower depends on the position of the tower and ranges form 3 to 28 . The granularity is

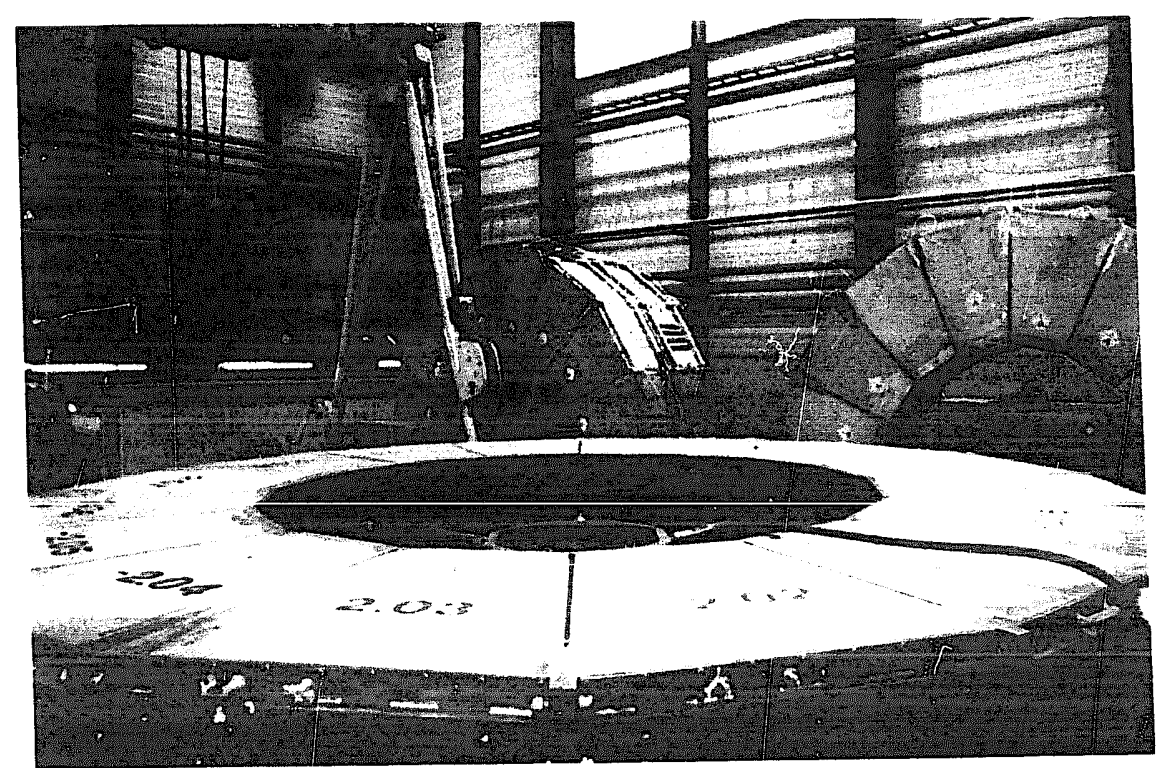

Fig. 57. An assembled hadron calorimeter ring. 


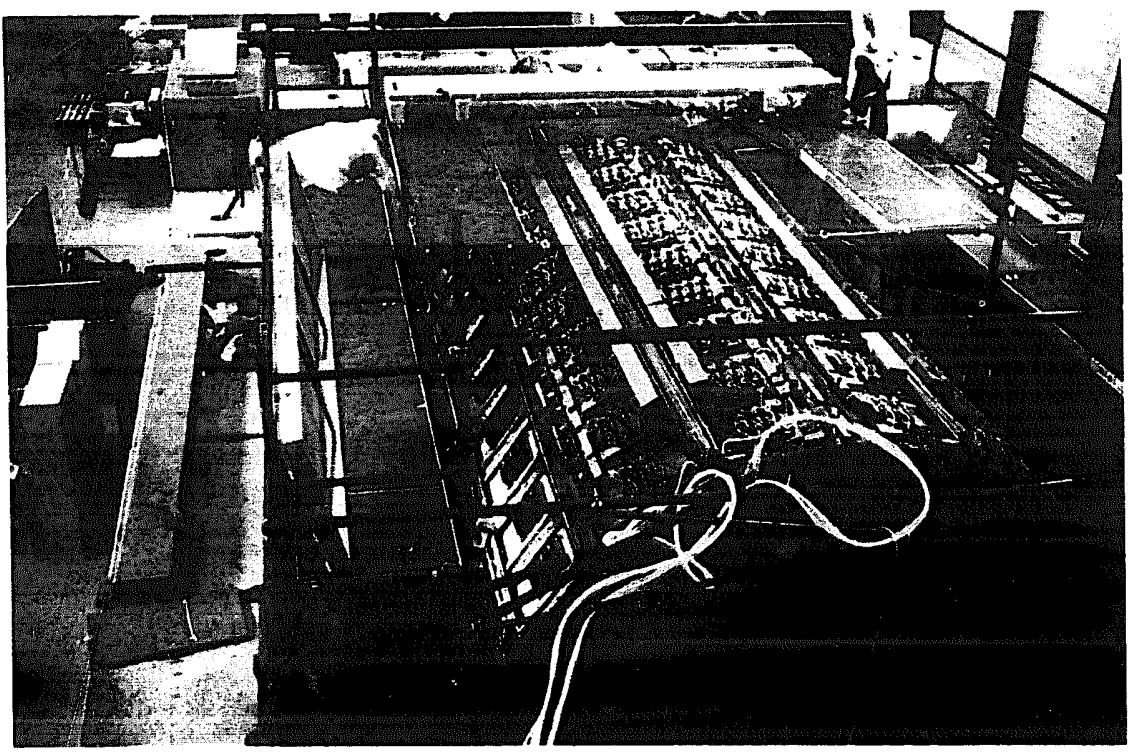

Fig. 58. The assembled hadron calorimeter barrel.

highest at the front end of the module where higher shower densities are expected.

\subsection{Hadron calorimeter readout and electronics}

At a working high voltage of $1.6 \mathrm{kV}$ the gas gain is about $10^{4}$. This gives rise to an anode signal of about 50 fC per minimum ionizing particle passing through the chamber. The signal wires within each tower are connected in parallel. The signals from the towers are brought via ribbon cables to connectors fixed to the base plate. The total number of charge sensitive readout channels in the barrel is 23040 .

Electronically, each channel consists of a preamplifier, a 40-m cable, a passive receiving network, and a charge integrating ADC (fig. 60). The charge integrating preamplifier is a joint development effort between L3 and LeCroy Corporation. A preamplifier board contains 24 channels as well as power regulators, srjark gaps and rest pulse injection circuits. The differ ential output signal is transmitted to the ADC over approxinuately $40 \mathrm{~m}$ of $110-\Omega$ twisted pair cables grouped into shielded bundles of 24 pairs. At the receiving end of the cable there is a transformer and an attenuation network which sets the energy scale in the ADC.

The ADC cinosen are the 96 channel LeCroy model 188212 bit FASTBUS ADC, with a $s^{\star}$ nsitivity of $50 \mathrm{fC}$ per count. The consersion plus rearout time is slightly more than $500 \mu \mathrm{s}$. The ADC are placed into FASTBUS crates in groups of 18 rogether with the Segment Manager module and Calibration module. Since the beam crossings occur every $22 \mu \mathrm{s}$, the ADC hold the charge on a capacitor before Jigitizing, until the first level trigger gives a decision if no first level trigger occurs, the ADC are cleared before the next beam crossing. In the event of a first level trigger the ADC digitize the data and store them in a multiple event buffer on the ADC board. In the meantime any event left over in the buffer from a previous gate is piped out to Multiple Record Buffer memories over 70-m ECL cables. Finally the data are moved to Dual Slave Memories for event building and for transfer to the VAX pending the second and third level trigger decisions.

The ADC provide immediately a fraction $(1 / 8)$ of the input charge on a separate line for trigyering purposes. These lines are grouped into 288 independent trigger signals which are digitized by Fast Encoding and Readout ADC. These data are then used by the energy and cluster triggers.

Table 3

Thickness in $\mathrm{cm}$ of calorimeter and other components measured normal to the beam line

\begin{tabular}{|c|c|c|}
\hline & $\begin{array}{l}\text { Long } \\
\text { modules }\end{array}$ & $\begin{array}{l}\text { Short } \\
\text { modules }\end{array}$ \\
\hline $\begin{array}{l}\text { Inner ring + inner flange } \\
\text { (Stainless steel) }\end{array}$ & 4.0 & 4.0 \\
\hline First plate (stainless steel) & 1.5 & 1.5 \\
\hline Base plate (stainless sie-i) & 1.5 & 1.5 \\
\hline Shield plates (brass) & 8.4 & 7.4 \\
\hline PWC tubes (brass) & 5.3 & 4.7 \\
\hline Uranium & 27.94 & 24.57 \\
\hline CHO : Mylar & 2.0 & 1.8 \\
\hline $\mathrm{BGO}$ & \multicolumn{2}{|c|}{24.0} \\
\hline Scintillator & \multicolumn{2}{|c|}{1.0} \\
\hline Muon filter (brass) & \multicolumn{2}{|c|}{16.5} \\
\hline Suppoit tube (stainless steel) & \multicolumn{2}{|c|}{10.0} \\
\hline
\end{tabular}




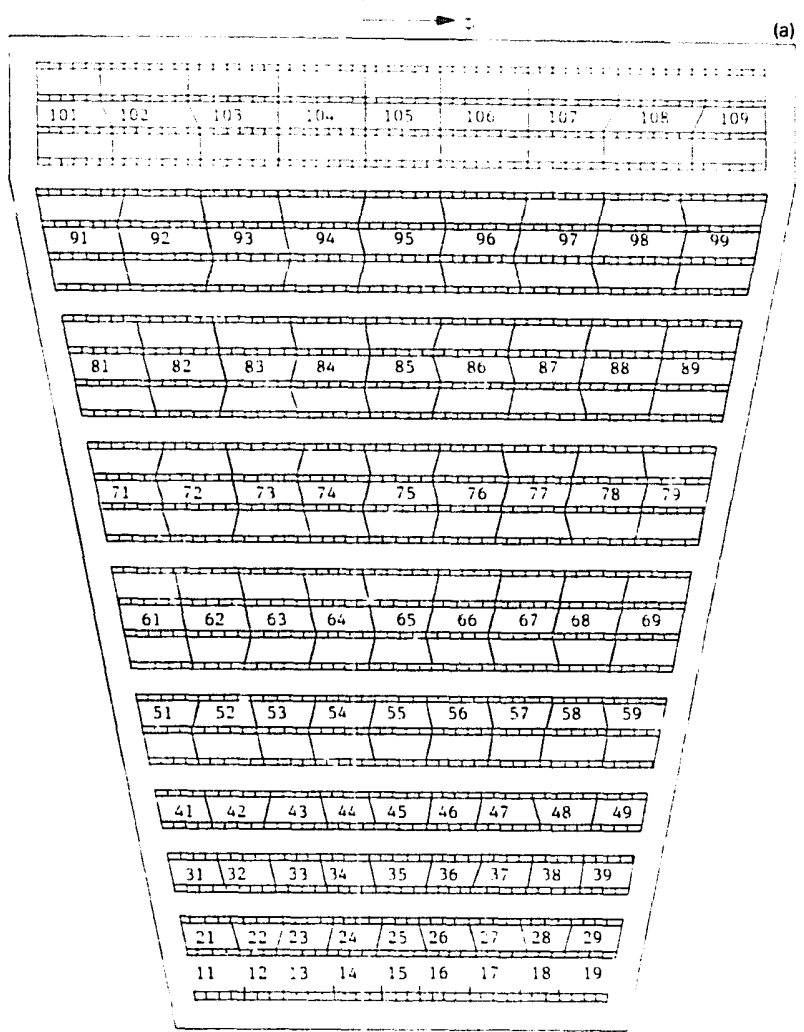

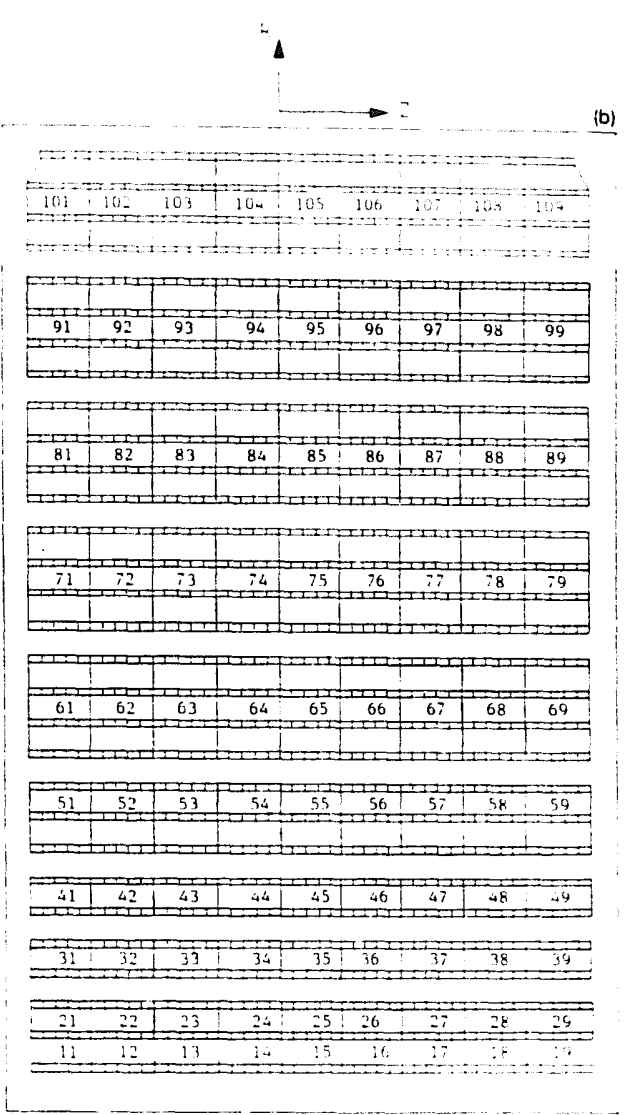

(a) $\Phi$ projection; (b) $Z$ projection.

Fig. 59. The wire grouping for the long module

\subsection{Physical properties}

The relevant properties of the barrel in terms of the thickness of various materials of which it is constructed are given in table 3 . In tables $4 a-4 c$ the thickness of the barrel components and the integral from the beam axis (perpendicular to the axis) are given in interaction lengths and minimum ionization energy loss. Values in table 4 are calculated from the data published by the Particle Data Group except for pion interaction data, which are taken from ref. [16]. Table 5 shows how the calorimeter thickness varies with the polar angle $\Theta$.

The properties of the hadron calorimeter utilizing $\mathrm{Ar} / \mathrm{CO}_{2}$ gas proportional chambers with uranium absorber piates have been studied extensively, both as prototypes and for compieted detector modules. One phase of the study was the characterization of the

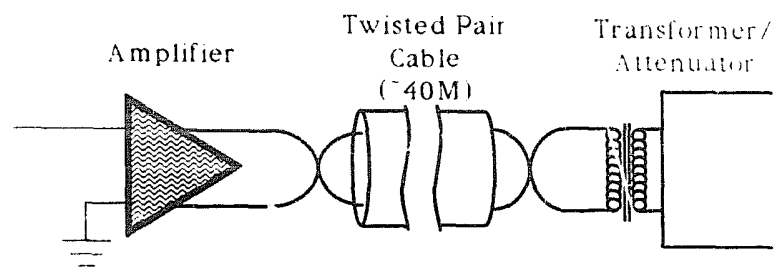

Fig. 60. Readout system for the hadron calorineter. response to cosmic ray muons; a typical response of a wire group to muons is given in fig. 61 . The response is in good agreement with the expectal distribution.

The response of prototypes and of the finished modulas to beams of hadrons and electrons was studied at the CERN and ITEP accelerators between 1 and 50 $\mathrm{GeV}$. A typical pulse height distribution for $20-\mathrm{GeV}$ pions is given in fig. 62 . The response as a function of energy is linear. The resolution, defined as the standard deviation of a Gaussian fit to the pulse height distribution, versus energy is shown in fig. 63, where data from prctotypes and completed modules ot tained both with and without BGO in front are used. Extensive studies have also beein made with other gas mixtures including isobutane in various mixtures [17].

\section{The hadron chicrimeter forward-backward system}

\subsection{Introduction}

The endcaps of the hadron calorimeter (HCEC) cover the folar angle regions $5.5^{\circ} \leq \Theta \leq 35^{\circ}$ and $145^{\circ} \leq \Theta$ $\leq 174.5^{\circ}$ over the full azimuthal range $0^{\circ} \leq \Phi \leq 360^{\circ}$. The solid angle covered by the endcaps ( $18 \%$ of $4 \pi$ ) extends the coverage of the hadronic calorimetry to 
$99.5 \%$ of $4 \pi$. Fig. 64 shows a longitudinal $(R-z)$ cut of the central detector, while fig. 65 shows a perspective view of the HCEC. The HCEC consist of three separate rings: an outer ring $(\mathrm{HCl})$ and two inner rings ( $\mathrm{HC} 2$ and $\mathrm{HC} 3$ ). Each ring is split vertically into half-rings, resulting in a total of 12 separate modules. The modularity of the HCEC detectors permits their fast withdrawal to provide access to the other L3 central

Table 4a

Hadron barrel thickness in units of nuclear interaction lengths for pions

\begin{tabular}{|c|c|c|c|c|c|c|}
\hline \multirow{2}{*}{$\begin{array}{l}\text { Material } \\
\text { BGO }\end{array}$} & \multicolumn{3}{|c|}{ Differential interaction length } & \multicolumn{3}{|c|}{ Integral interaction length } \\
\hline & & 0.93 & & & & \\
\hline Scintillator & & 0.01 & & & 0.94 & \\
\hline HC barrel & long & & short & long & & short \\
\hline Fe:(stainless steel) & 0.37 & & 0.37 & & & \\
\hline $\mathrm{Cu}:$ (brass) & 0.70 & & 0.62 & & & \\
\hline $\mathrm{U}:$ (absorber) & 2.42 & & 2.13 & & & \\
\hline CHO: Mylar & 0.03 & & 0.03 & 4.46 & & 4.09 \\
\hline Calorimeter subtotal & 3.52 & & 3.15 & & & \\
\hline Muon filter $(\mathrm{Cu})$ & & 1.03 & & 5.49 & & 5.12 \\
\hline Support tube (stainless steel) & & 0.52 & & 6.01 & & 5.64 \\
\hline
\end{tabular}

Table 4b

Hadron barrel thickness in units of radiation lengths

\begin{tabular}{|c|c|c|c|c|c|}
\hline Material & Differential radiation le & & Integral & n length & \\
\hline$\overline{\mathrm{BGO}}$ & 21.43 & & & 21.43 & \\
\hline Scintillator & 0.02 & & & 21.45 & \\
\hline HC barrel & long & short & long & & short \\
\hline Fe:(stainless steel) & 3.22 & 3.22 & & & \\
\hline $\mathrm{Cu}:$ (brass) & 8.61 & 7.61 & & & \\
\hline $\mathrm{U}:$ (absorber) & 87.31 & 76.78 & & & \\
\hline CHO: Mylar & 0.07 & 0.06 & 120.70 & & 109.16 \\
\hline Calorimeter subtotal & 99.25 & 87.71 & & & \\
\hline Muon filter (brass) & 11.54 & & i 32.24 & & 120.70 \\
\hline Support tube (stainless steel) & 5.78 & & 138.02 & & 126.48 \\
\hline
\end{tabular}

Table $4 c$

Hadron barrel energy loss for mind ionizing particles in $\mathrm{MeV}$

\begin{tabular}{|c|c|c|c|c|c|c|}
\hline \multirow{2}{*}{$\frac{\text { Material }}{\text { BGO }}$} & \multicolumn{3}{|c|}{ Differential energy loss } & \multicolumn{2}{|c|}{ Integral energy loss } & \\
\hline & & 221 & & & 221 & \\
\hline Scintillator & & 2 & & & 223 & \\
\hline $\mathrm{CH}$ & & & & & & \\
\hline HC barrel & long & & short & iong & & short \\
\hline $\mathrm{Fe}:($ stainless steel) & 83 & & 83 & & & \\
\hline $\mathrm{Cu}:$ (brass) & 159 & & 140 & & & \\
\hline $\mathrm{U}:$ (absorber) & 578 & & 509 & & & \\
\hline CHO: Mylar & 5 & & 5 & 1048 & & 960 \\
\hline Calorimeter subtotal & 825 & & 737 & & & \\
\hline Muon filter $(\mathrm{Cu})$ & & 213 & & 1261 & & 1173 \\
\hline Support tube (stainless steel) & & 118 & & 1379 & & $12 \Omega_{1}$ \\
\hline
\end{tabular}


Table 5

Detector thickness at different angles in units of nuclear absorption lengths for pions

\begin{tabular}{|c|c|c|c|c|c|c|}
\hline$\Theta$ & BGO & $\mathrm{HB}^{\text {a) }}$ & $\mathrm{HC}^{\mathrm{b})}$ & $\mathrm{MF}^{\mathrm{C})}$ & $S T^{d)}$ & Total \\
\hline 90.0 & 0.94 & 3.52 & - & 679 & 0.52 & 5.97 \\
\hline 82.0 & 0.94 & 3.56 & - & 100 & 0.52 & 6.02 \\
\hline 72.5 & 0.94 & 3.70 & - & 1.34 & 0.54 & 6.22 \\
\hline 66.5 & 0.94 & 3.84 & - & 1.08 & 0.54 & 6.42 \\
\hline 58.5 & 0.94 & 4.28 & - & 1.16 & 0.60 & 6.98 \\
\hline 54.0 & 0.94 & 4.56 & - & 0.49 & 0.64 & 6.63 \\
\hline 50.5 & 0.94 & 4.54 & - & 0.52 & 0.67 & 6.67 \\
\hline 48.5 & 0.94 & 4.35 & - & 0.25 & 0.69 & 6.23 \\
\hline 42.5 & - & 4.92 & - & - & 0.75 & 5.68 \\
\hline 39.0 & - & 5.22 & - & - & 0.41 & 5.63 \\
\hline 35.0 & - & 5.12 & 1.05 & - & 0.45 & 6.62 \\
\hline 32.0 & - & 3.74 & 1.68 & - & 0.48 & 5.90 \\
\hline 27.0 & - & 1.97 & 3.13 & - & 0.47 & 5.67 \\
\hline 22.0 & - & - & 6.36 & - & - & 6.36 \\
\hline 17.0 & - & - & 6.01 & - & - & 6.01 \\
\hline 12.0 & - & - & 5.91 & - & - & 5.91 \\
\hline
\end{tabular}

a) HB: hadron calorimeter barrel.

b) HC: hadron calorimeter endcaps.

c) MF: muon filter.

d) ST: support tube.

xxxxx

detector components. The $\mathrm{HCl}$ half-rings are separated at $\Phi=90^{\circ}\left(270^{\circ}\right)$ by $10-\mathrm{mm}(40-\mathrm{mm})$ slits. The $10-\mathrm{mm}$ slit accommodates the support of the LEP beam pipe, while the $40-\mathrm{mm}$ one allows for passage of the vacuum tube of an RFQ device for BGO crystal calibration.

\subsection{Technical descriptio: of the hadron calorimeter end-} caps

The endcaps consist of stainless steel containers filled with alternating layers of brass tube proportional chambers (inner tube dimensions $5 \mathrm{~mm} \times 10 \mathrm{~mm}$ ) and $5-\mathrm{mm}$ and 10-mm thick absorber plates of depleted uranium (fine and coarse sampling part respectively). Over the end cap region, the amount of material traversed by a

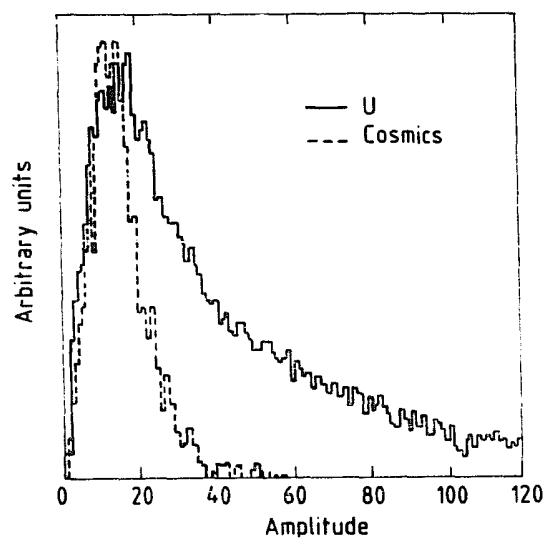

Fig. 61. Calorimeter response to cosmic ray muons.

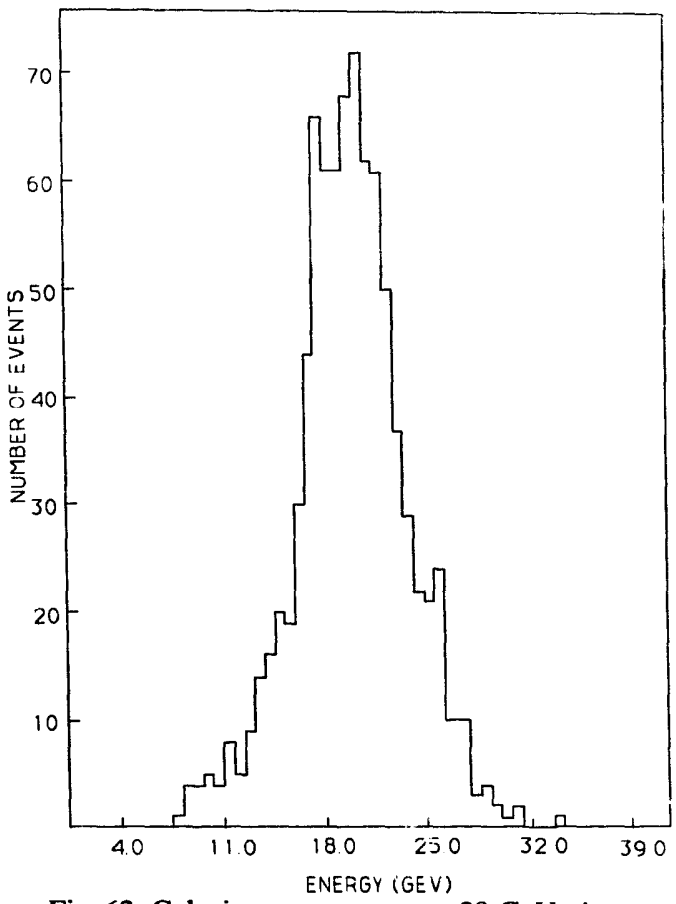

Fig. 62. Calorimeter response to $20 \mathrm{GeV}$ pions.

particle originating at the interaction point varies between 6 and 7 nuclear absorption lengths.

\subsubsection{Containers}

The $\mathrm{HCl}$ containers are subdivided into four compartments by $16-\mathrm{mm}$ thick stainless steel walls providing structural rigidity. Their thickness is chosen to ap-

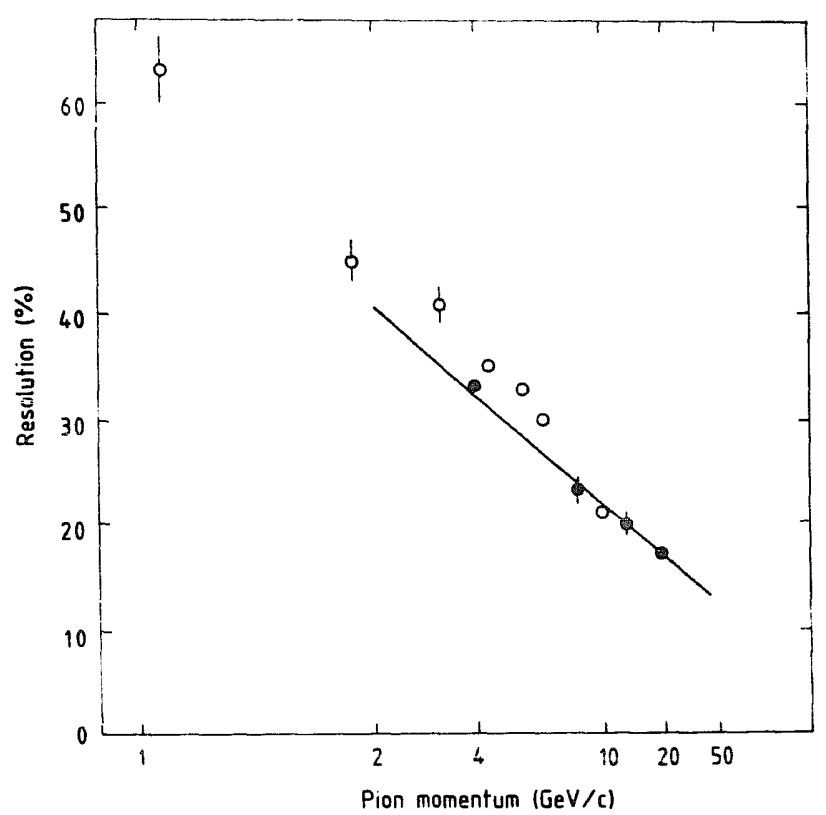

Fig. 63. Hadron calorimeter energy resolution. The line is drawn through the points obtained with the BGO in front (full circles) and corresponds to $\sigma / E=(55 / \sqrt{E+5})^{\%}$. 


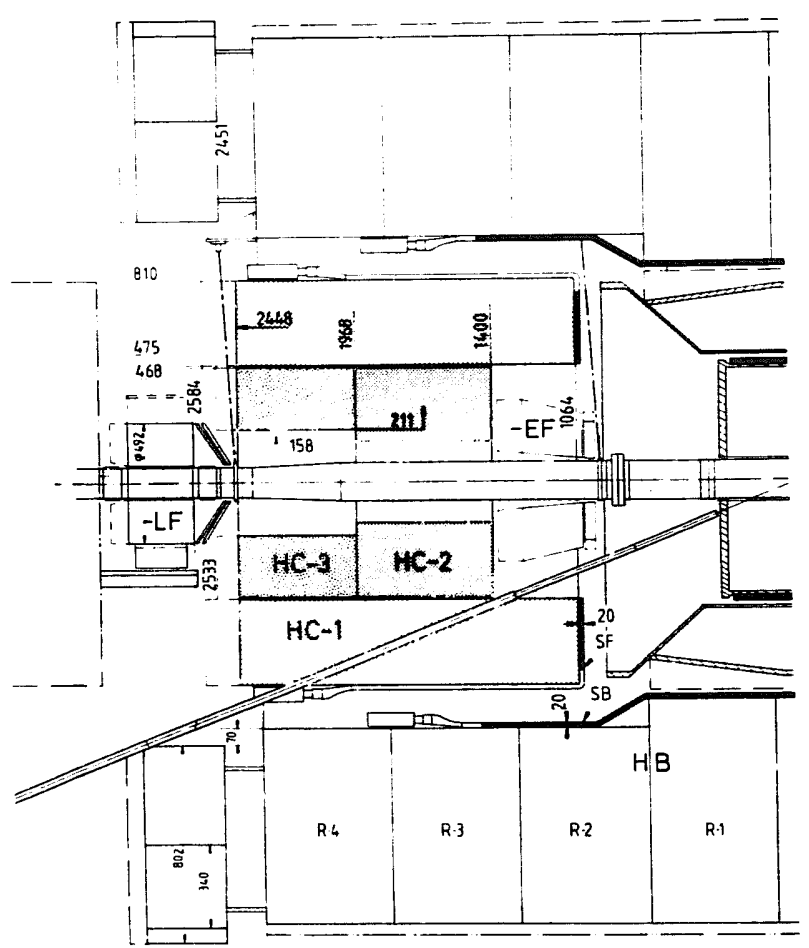

Fig. 64. Longitudinal cut of part of the L3 hadron calorimeter. One side of the forward-backward system is shown (dotted area).

proximate that of a $10-\mathrm{mm}$ thick uranium plate in terms of nuclear absorption lengths. The first compartment (fine sampling part) is 26 chamber layers deep and is equipped with 5-mm uranium plates. In this compartment the first three layers of uranium plates have been
Table 6

Dimensions and mechanical properties of the hadron calorimeter endcap modules

\begin{tabular}{lllll}
\hline & \multicolumn{2}{l}{ Container type } & & Total \\
\cline { 2 - 4 } & HC1 & HC2 & HC3 & \\
system
\end{tabular}

replaced by steel plates in order to shield the BGO crystals and the TEC chamber from the uranium radioactivity background. The three remaining compartments, eacn 17 chamber layers deep, are equipped with

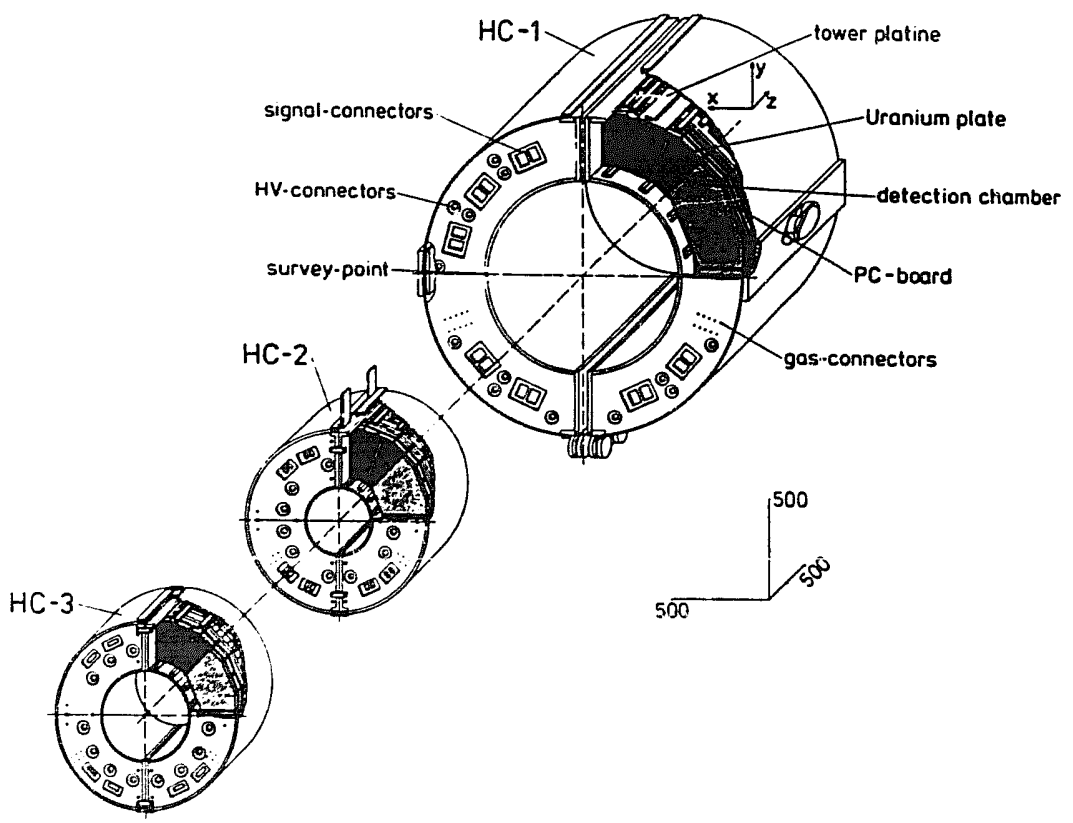

Fig. 65. Perspective view of the forward-backward hadron calorimeter. 
$10-\mathrm{mm}$ thick uranium plates (two 5 -mm plates put together). There are thus 77 chamber layers in the $\mathrm{HC} 1$ container. The $\mathrm{HC} 2$ and $\mathrm{HC} 3$ containers, with a depth of 27 and 23 chamber layers respectively, are subdivided into two compartments each, again via $16-\mathrm{mm}$ thick stainless steel walls. All compartments are equipped with $10-\mathrm{mm}$ thick absorber plates. As for $\mathrm{HC} 1$, shielding considerations led to the replacement of the first $15 \mathrm{~mm}$ of uranium in $\mathrm{HC} 2$ by the same thickness of steel. Table 6 summarizes the dimensions and mechanical properties of the $\mathrm{HCl}, \mathrm{HC} 2$ and $\mathrm{HC} 3$ containers.

\subsubsection{Proportional chambers}

Within a half-ring, a chamber layer consists of four chambers, each covering an inte val $\Delta \Phi=45^{\circ}$ (U-layer). The wires are stretched azimuthally to measure the polar angle $\Theta$ directly. Even numbered chamber layers (V-Layers) are rotated by $\Delta \Phi=22.5^{\circ}$ with respect to the odd numbered ones. This stereo angle between successive layers allows measurement of the coordinate $\Phi$ orthogonal to $\Theta$ and the gaps between chambers do not coincide in successive layers. Chambers whose wires would have crossed the boundary between two half-rings are split into two halves. Every second chamber layer is thus comprised of three full-sized chambers and two half-sized ones.

Details of the construction of the proportional tube chambers are illustrated in fig. 66 . The individual brass tubes have an inner cross section of $5 \times 10 \mathrm{~mm}^{2}$ and a wall thickness of $0.3 \mathrm{~mm}$. The chamber is shielded on both sides against uranium irradiation by $0.7-\mathrm{mm}$ thick brass plates. To save the space required by decoupling capacitors, high voltage is applied to the body of the tubes rather than to the wires. A $0.2-\mathrm{mm}$ thick polycarbonate foil, glued between the tubes and the outer shielding plates, provides the necessary insulation. End regions are additionally protected against high voltage breakdowns by Kapton foil. Each tube contains a 50- $\mu \mathrm{m}$ diameter gold-plated tungsten wire, siretched with a 200-g tension ard crimped on a gold-plated $\mathrm{Cu}-\mathrm{Be}$ piece fixed inside the plastic chamber endpiece. On one side of the chamber, printed circuit boards, soldered to pins on the $\mathrm{Cu}-\mathrm{Be}$ wire fixation pieces, serve to transmit the wire signals to the outside world. Within a chamber, serial circulation of the gas from one tube to the next is achieved via gas throughputs milled inside the plastic endpieces.

Chambers for the $\overline{\mathrm{HCl}}, \overline{\mathrm{HC}} \mathrm{2}$ and $\overline{\mathrm{HC}} 3$ rings consist of 25, 24 and 19 tubes respectively. Overal!, the HCEC detector contains 2284 individual chambers with a total of 54140 wires (table 6).

\subsubsection{Absorber}

The azimuthal segmentation of the absorber is twice that of the chambers. fig. 67 shows the arrangement of a)
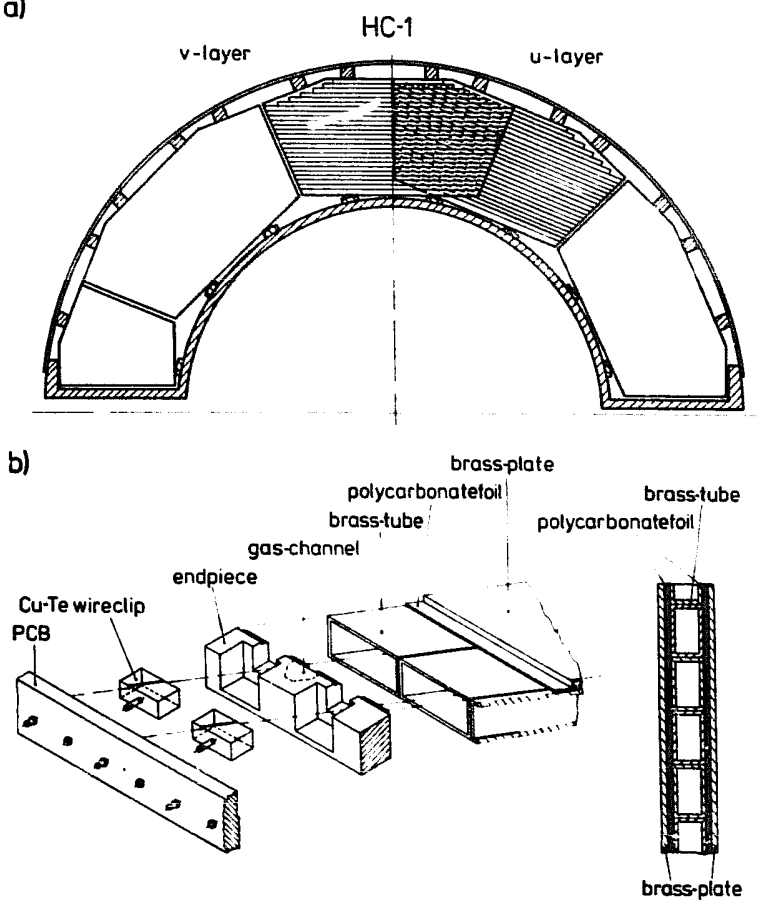

Fig. 66. Hadron calorimeter forward-backward system. (a) Shows schematically the chamber arrangement with the $22.5^{\circ}$ stereo angle of the wires. (b) Shows details of the chamber construction.

the depleted uranium plates within a $\mathrm{U}$ or $\mathrm{V}$ absorber layer of a $\mathrm{HCl}$ and $\mathrm{HC} 2$ half-ring. (An absorber layer is defined to be of the $\mathrm{V}$ type, when it follows a $\mathrm{V}$ type chamber laye-). The gaps between plates do not coincide in successive layers and they do not point to the beam axis; nor do they coincide with the gaps between chambers (compare figs. 66 and 67; see also fig. 65).

\subsubsection{Wire grouping}

The wire signals are grouped to form "towers" pointing to the interaction region. In the $R-z$ plane the

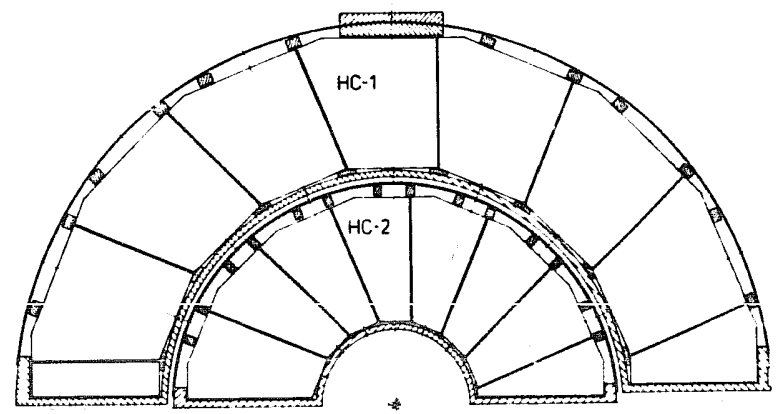

Fig. 67. Hadron calorimeter forward-backward system. The figure shows the uranium absorber plate arrangement for the $\mathrm{HC} 1$ and $\mathrm{HC} 2$ modules. Displayed is the arrangement for the $\mathrm{U}$-layers. For the V-layers the plate arrangement is mirror symmetric. 
detector is segmented in $\mathbf{3 1}$ pointing "roads" of width $\Delta \Theta \approx 1^{\circ}$ by grouping two wires (or their equivalent) at the $z$ position of the first $\mathrm{HCl}$ chamber layer. Signal "towers" are then formed by subdividing each of the three end cap rings in depth and grouping the wires within each road. To retain the stereo angle information, separate towers are formed for the U- and V-type layers. The azimuthal segmentation is therefore $\Delta \Phi=$ $22.5^{\circ}$. In depth, $\mathrm{HC} 1$ is divided into a total of seven logical segments (fig. 68), the first two forming the fine sampling part, while HC2 and HC3 are divided into three and two segrnents respectively. This segmentation scheme results in a total of 3960 signal towers for the two endcaps. The tower structure of the HCEC is summarized in table 7 .

\subsubsection{Services}

High voltage is provided to the chambers by 72 supply channels, which are fanned out into a total of 180 lines. Each line is connected to a group of 11 to 14 chambers. Distribution boxes, mounted on the back flanges of the ditector, allow us to disconnect individual chambers from the high voltage system, e.g. if a particular chamber draws excessive current.

Gas is supplied to the chambers via four input lines feeding a total of 20 circuits for the $\mathrm{HCl}$ containers and 4 each for HC2 and HC3. Chambers served by the same circuit are connected serially to the gas supply. There are between 38 and 128 chambers per circuit because, due to the various chamber sizes within the HCEC, it was possible to equalize to within $30 \%$ the resistance of
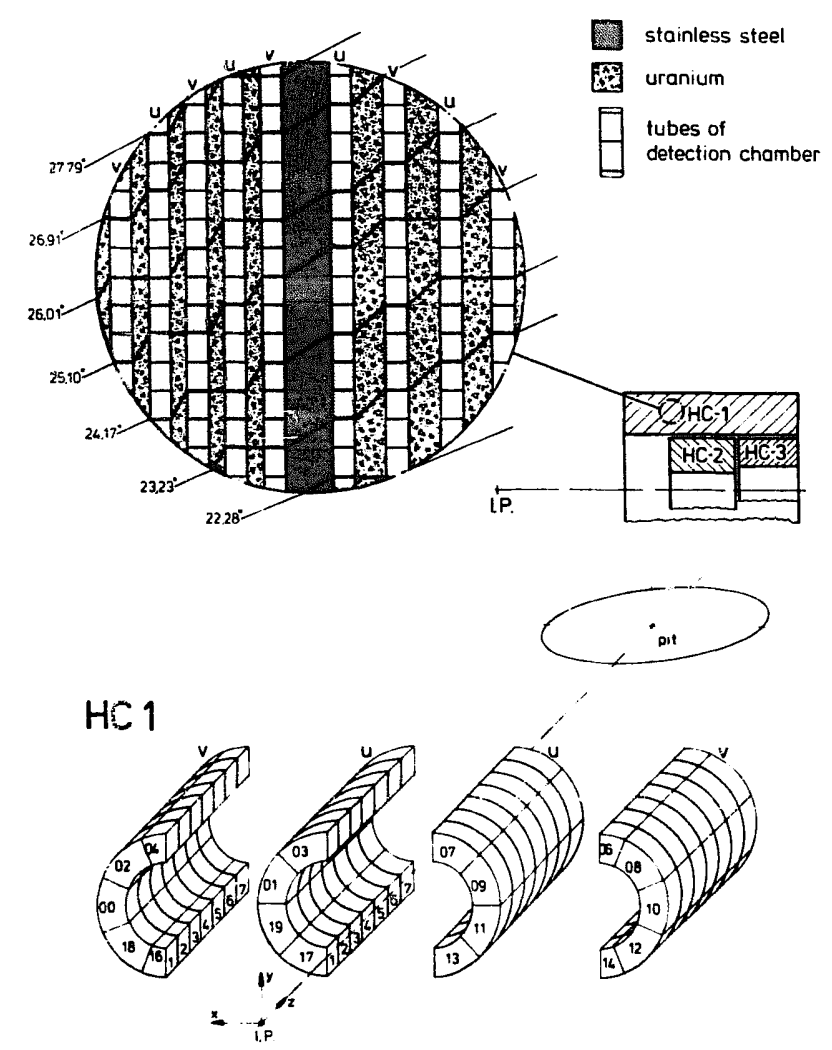

Fig. 68. Hadron calorimeter forward-backward system. In the lower part of the figure the segmentation of the $\mathrm{HCl}$ containers in $\Phi$ and $z$ are shown separately for the U- and $V$-layers. The upper part of the figure shows enlarged the $\Theta$ segmentation into roads at the border of the fine and coarse sampling parts.

Table 7

Segmentation (tower structure) of the hadron calorimeter end caps

\begin{tabular}{|c|c|c|c|c|c|c|c|c|}
\hline & \multicolumn{7}{|c|}{ Longitudinal segment } & \multirow[t]{2}{*}{ All } \\
\hline & $\overline{1}$ & 2 & 3 & 4 & 5 & 6 & 7 & \\
\hline \multicolumn{9}{|l|}{$\overline{\mathrm{HCl}}$} \\
\hline Number of U-chr layers & 7 & 6 & 5 & 4 & 5 & 6 & 6 & 39 \\
\hline Number of V-chr layers & 6 & 7 & 4 & 5 & 4 & 6 & 6 & 38 \\
\hline Number of towers $(U+V)$ & 520 & 468 & 396 & 344 & 324 & 288 & 252 & 2592 \\
\hline Number of amplifier boards & & & & & & & & 108 \\
\hline Number of ADC modules & & & & & & & & 28 \\
\hline \multicolumn{9}{|l|}{$\mathrm{HC} 2$} \\
\hline Number of U-chr layers & & & 4 & 5 & 5 & & & 14 \\
\hline Number of V-chr layers & - & - & 5 & 4 & 4 & - & - & 13 \\
\hline Number of towers $(U+V)$ & & & 344 & 324 & 288 & & & 956 \\
\hline Number of amplifier boards & & & & & & & & 40 \\
\hline Number of ADC modules & & & & & & & & 10 \\
\hline \multicolumn{9}{|l|}{$\mathrm{HC} 3$} \\
\hline Number of U-chr layers & & & & & & 6 & 6 & 12 \\
\hline Number of V-chr layers & & & & & & 5 & 6 & 11 \\
\hline Number of towers $(U+V)$ & & & & & & 232 & 180 & 412 \\
\hline Number of amplifier boards & & & & & & & & 20 \\
\hline Number of ADC modules & & & & & & & & 5 \\
\hline
\end{tabular}


the various circuits. The half-ring containers themselves, excluding the chamber volume, can also be flushed serially (i.e. one gas line per end cap) either with the counting gas or with an inert gas (e.g. $\mathrm{CO}_{2}, \mathrm{~N}_{2}$ ). At the chamber outlet, the gas density is stabilized using the signals of pressure transducers and temperature probes. For temperature monitoring, $84 \mathrm{Pt} 100$ probes are mounted on chambers located at top, bottom and along the centerline of each half-ring container.

\subsubsection{Readout and trigger segmentation}

The 3960 tower signals are amplified by 168 LRS 2724 preamplifier boards mounted on the rsar flanges of the $\mathrm{HCl}$ and $\mathrm{HC} 3$ containers. The signals are then fed through an approximately 55-m long w id pair cable to 43 LRS 1885F FASTBUS ADC modules located in the blockhouse (table 7).

For triggering purposes the hadron calorimeter end cap modules are longitudinally divided into two trigger planes: $A(1.5 \lambda)$ and $B$ for the remainder (up to $6.5 \lambda$ ). Each plane is azimuthaly $(\Phi)$ subdivided into 16 elements while the polar $(\Theta)$ subdivision contains 4 elements.

\subsection{Detector performance}

\subsubsection{Proportional chamber performance}

The chambers are operated with an $80 \%$ argon-20\% $\mathrm{CO}_{2}$ gas mixture. The single layer signal for a minimum ionizing particle at normal incidence is $100 \mathrm{fC}$ with a charge collection time of $240 \mathrm{~ns}(95 \%)$. At an operating high voltage of $1650 \mathrm{~V}$ the gas amplification is $1.5 \times 10^{4}$. In fig. 69 the mean pulse height distribution for all 2284 chambers mounted into the endcaps is displayed showing an excellent manufacturing homogeneity of $\delta /$ mean of $3 \%$. The sensitivity due to changes of the environ-

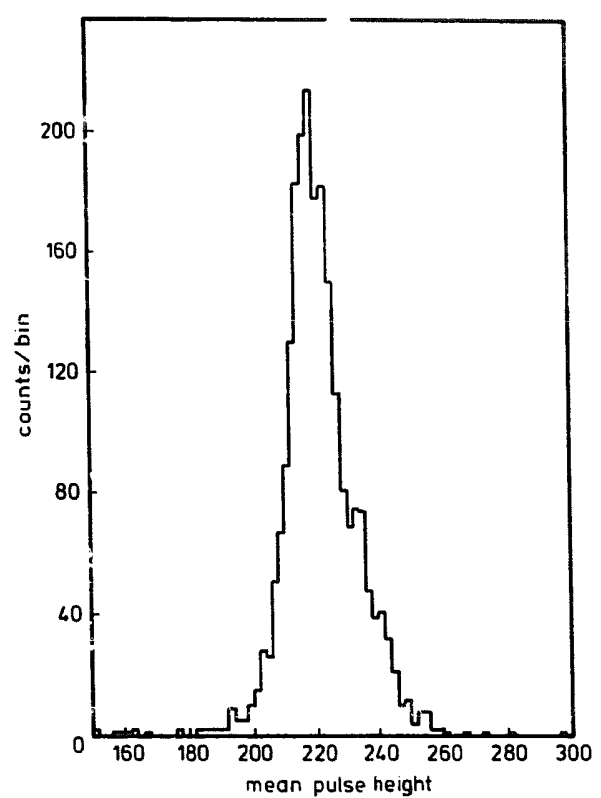

Fig. 69. Hadron calorimeter forward-backward system. Mean pulse height distribution for all 2284 chambers mounted into the endcaps $(\delta /$ mean $=0.03)$ measured with cosmic muons.

ment is as follows:

$$
\begin{array}{ll}
\text { Pressure: } & \Delta Q /(Q \Delta p)=0.6 \% \mathrm{mbar}^{-1} ; \\
\text { Temperature: } & \Delta Q /(Q \Delta T)=1.8 \% \mathrm{C}^{-1} ; \\
\text { High voltage: } & \Delta Q /(Q \Delta U)=1.2 \% \mathrm{~V}^{-1} ;
\end{array}
$$

Particular attention was given to the long term behavior of the proportional chambers under radioactive load of the uranium absorber. An accelerated aging test has been carried out with a strong ${ }^{129} \mathrm{Ru}$ source. No change in the performance within $3 \%$ has been found up to a corresponding lifetime of $800 \mathrm{yr}$ in the uranium environmerı of the end caps (fig. 70).

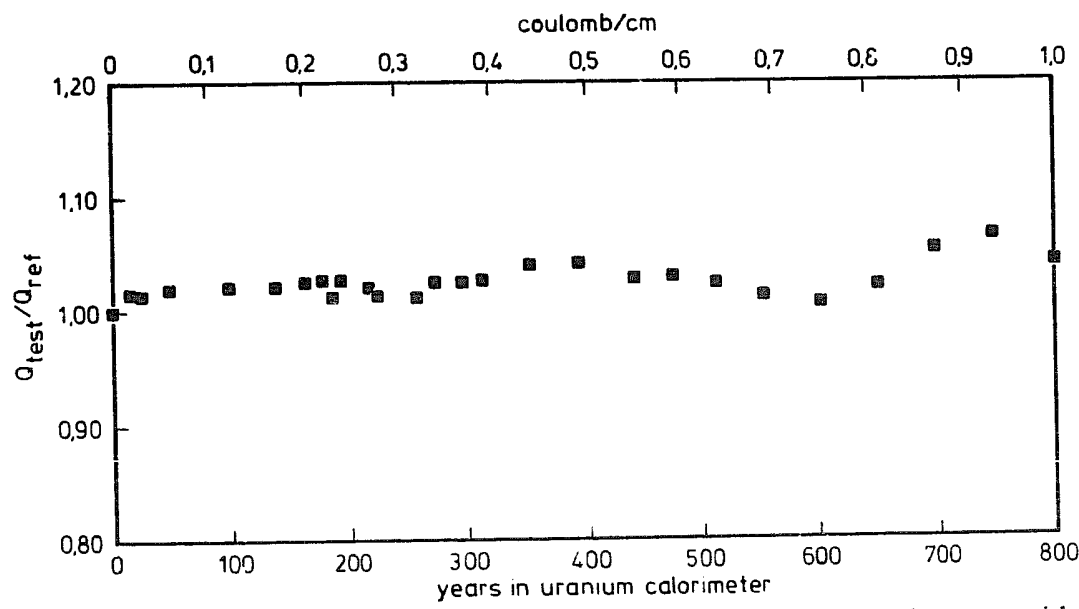

Fig. 70. Hadron calorimeter end cap chamber behavior under irradiation. Accelerated aging test with ${ }^{129}$ Ru. Normal chamber operation conditions (see text). $Q_{\text {ref }}$ is the signal from ${ }^{55} \mathrm{Fe}$ taken in the non-irradiated part of the chamber. $Q_{\text {test }}$ is the signal at the center of irradiation. The linear charge density of $1 \mathrm{C} / \mathrm{cm}$ accumulated in 30 days of ${ }^{129} \mathrm{Ru}$ irradiatior corresponds to 800 years of operation in the $\mathrm{L} 3$ experiment. 


\subsubsection{Calibration and monitoring}

An absolute calibration of the calorimeter with cosmic muons has been performed using scintillation trigger counters above and below the end cap modules. Additional concrete shielding resulted in a lower muon cutoff energy of $2 \mathrm{GeV}$. A face to face cut was applied to the raw data, demanding a coincidence of three consecutive vertically arranged towers to ensure that the center tower was traversed by the muon vertically in full length. Preliminary results for one half-ring arrangement (one $\mathrm{HC} 1, \mathrm{HC} 2$ and $\mathrm{HC} 3$ module) are shown in fig. 71. The mean pulse height of the center towers corresponds to 45 ADC channels with a variance of $8 \%$.

The monitoring and relative calibration of the hadron calorimeter end caps will be achieved by using the radioactive background radiation of the uranium absorber. The total ionization yield ife to electrons and photons ( $\alpha$ 's are absorbed in the chamber covers) can be measured accurately with our gaseous detector, although we cannot detect a single photon line from the chain of radioactive decays of the uranium absorber nuclei. In our chamber/absorber arrangement this ionization rate amounts to $40 \mathrm{~Hz} / \mathrm{cm}^{2}$ yielding $10^{4}$ counts $/ \mathrm{s}$ for a typical tower. At this high rate we can randomly capture uranium signals with high efficiency while opening the ADC for $500 \mathrm{~ns}$ with $2-\mathbf{k H z}$ repetition rate. This mode of operation is highly practical in our 4000 channels system as we avoid additional circuitry for the calibration gate formation. The approximately exponential spectrum of the ionization yield observed in this fashion is shown in fig. 72; At higher chamber gain, the slope of the spectrum decreases while its end point is

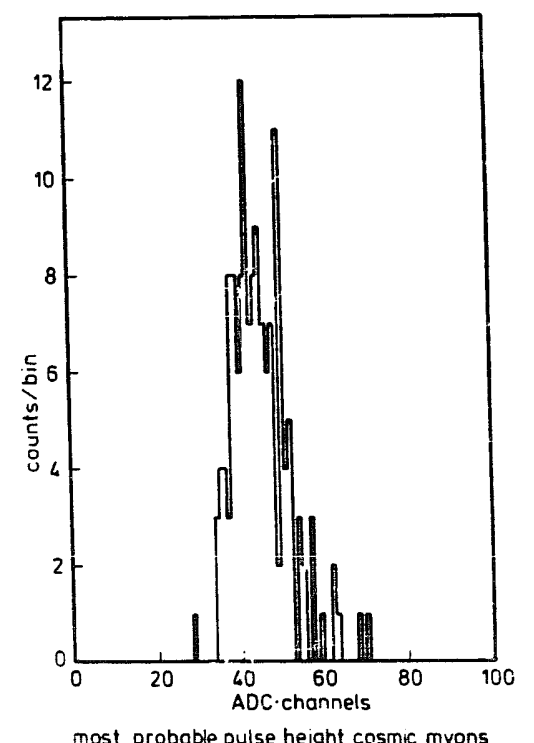

Fig. 71. Hadron calorimeter forward-backward system. Mean pulse height distribution obtained with cosmic muons for a fully assembled arrangement of a $\mathrm{HC} 1, \mathrm{HC} 2$ and $\mathrm{HC} 3$ container after applying a face tc face cut to the raw data.

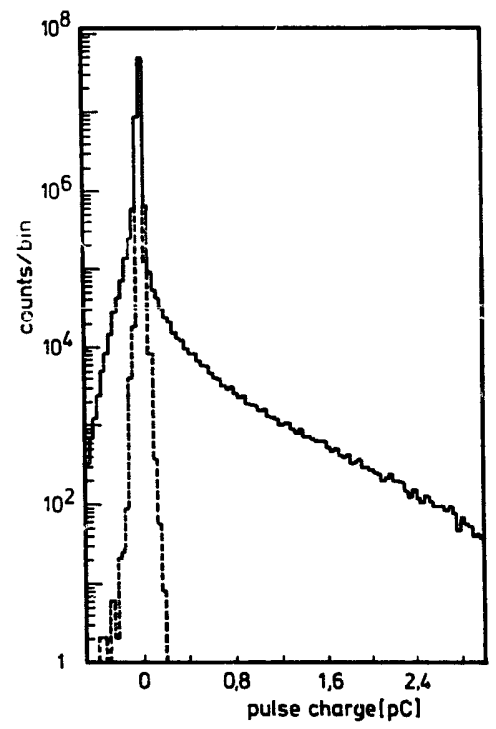

Fig. 72. Pulse height distribution of U-noise signals. About $10^{-3}$ of the gate openings capiure a uranium signal. Electromagnetic pickup noise is small as demonstrated by the dashed distribution, observed while lowering the high voltage by $300 \mathrm{~V}$ from the standard operation point.

pushed further out. This fact is used to correct for any changes of the detector gain due to environmental changes (gas temperature, pressure, $\mathrm{HV}$, etc.).

Spectra $f_{i}$ are taken at regular intervals (during run startup or end) and compared to a reference spectrum $r$. The actual gain $g$ is determined from the minimum of the $\chi^{2}$ function,

$$
\begin{aligned}
& \chi^{2}(g, k)=\sum_{i}\left(f\left(g x_{i}\right)-\frac{k}{g} r\left(x_{i}\right)\right)^{2} / \delta^{2}, \\
& k=\frac{F}{R}=\int_{g x_{\min }}^{\infty} f(x) \mathrm{d} x / \int_{x_{\min }}^{\infty} r(x) \mathrm{d} x,
\end{aligned}
$$

which is a measure of the similarity between the test spectrum $f$ and the reference spectrum $r$. By comparison to directly measured spectra from ${ }^{55} \mathrm{Fe}$ we have established that this calibration method works reliably for gain changes within a factor of five (fig. 73).

The variances of the gain $g$ determined from the $x^{2}$ minimization is inversely proportional to the number of uranium signals observed (fig. 74). The gain test spectrum information is recorded at full readout speed. A 3\% precision run corresponding to 3000 entries takes approximately $150 \mathrm{~s}$. The limited knowledge of the reference spectrum determines the ultimate precision of this gain test method. With one day of running, reference spectra for $0.3 \%$ accurate gain tusts can be obtained.

Each wire contributes proportionally to its length to the integral $F$ of the uranium srectrum (fig. 75) of a detector cell ("tower") and the detector performance can be monitored at the wire level. 


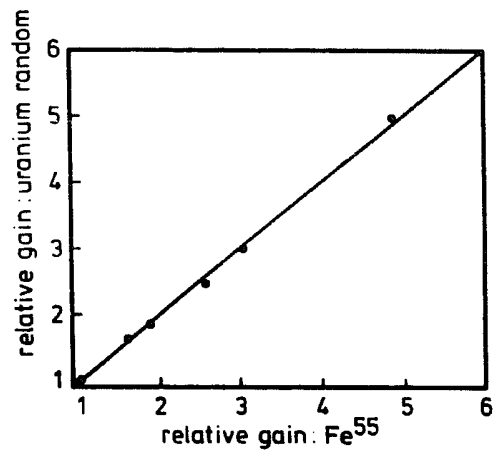

Fig. 73. Gain determination by observing uranium noise signals compared to gain determination by observing the maximum of the pulse height distribution from ${ }^{55} \mathrm{Fe} 5.9 \mathrm{keV}$ gamma rays in the same chamber. The gain was varied by changing the high voltage.

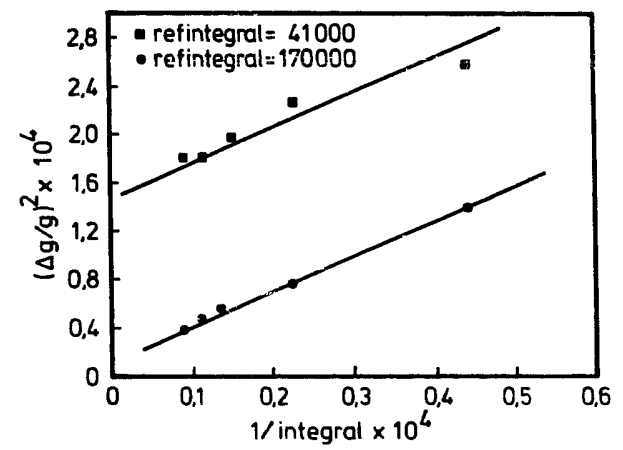

Fig. 74. Precision of the gain determination as a function of the inverse number of entries in the randomly sampled $U$ spectrum. With high statistics in the reference spectrum relative gain determinations to better than $1 \%$ can be achieved

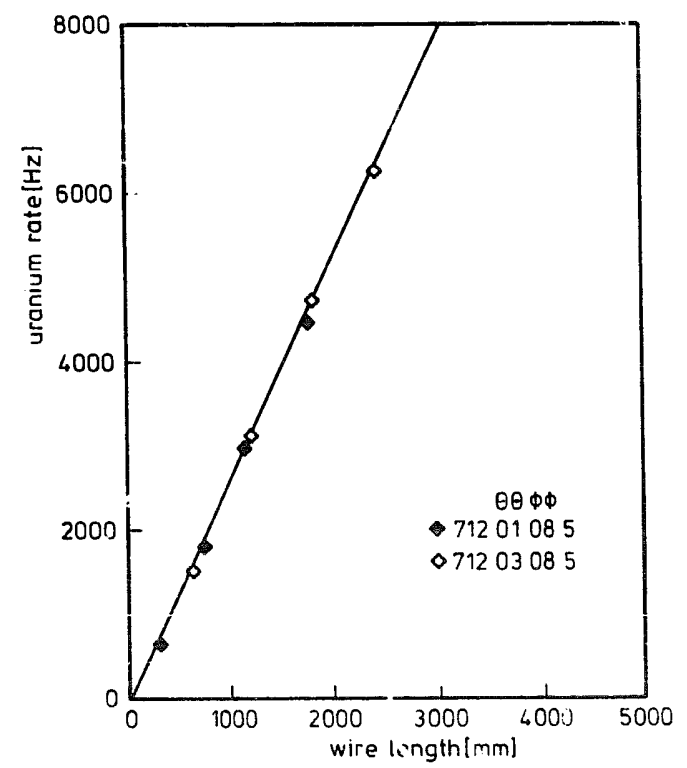

Fig. 75. U rate for two different $\mathrm{HC} 2$ towers as a function of the operative length of the sense wire (change in length by connecting the appropriate chambers to high voltage selectively). The very low end of the $U$ spectrum $\left(Q_{U} \leq Q_{\text {mip }}\right)$ has been cut out resulting in a slope of $26 \mathrm{~Hz} / \mathrm{cm}$.

\subsubsection{Detector response to pions and electrons}

The response of the hadron calorimeter endcaps to pions up to $25 \mathrm{GeV}$ and electrons up to $10 \mathrm{GeV}$ has been studied in a prototype setup which had a simfilar internal structure but !llowed for larger shower containment [18]. The results are compared with Monte Carlo simulation calculations [19] giving good agreement with the data.

\section{The muon filter}

The muon filter is mounted on the inside wall of the support tube and adds 1.03 absorption length to the hadron calorimeter. It consists of eight identical octants, each made of six $1-\mathrm{cm}$ thick brass $(65 \% \mathrm{Cu}+35 \%$ $\mathrm{Zn})$ absorber plates, interleaved with five layers of proportional chambers and followed by five $1.5-\mathrm{rm}$ thick absorber plates matching the circular shape of the supporting tube (fig. 76). Each octant is 4-m long, 1.4-m wide and $0.2-\mathrm{m}$ thick in the radial direction. The first four layers of an octant each contain 16 chambers whereas the outermost layer contains 14 chambers. The muon filter proportional chambers are made of a 4-m long comb-like brass profile covered with a brass lid, thus forming eight tubes, and are enclosed in a plastic box to ensure gas tightness. Each tube is $8.4 \mathrm{~mm}$ wide and $5 \mathrm{~mm}$ high and is equipped with a resistive (470 $\Omega / \mathrm{m}$ ) wire $50 \mu \mathrm{m}$ in diameter. The tubes are separated by $1.6-\mathrm{mm}$ thick walls. They are extruded industrially; wires are strung manually and supported every $20 \mathrm{~cm}$ by plastic holders. The chambers are terminated by a hoard equipped with eight $700 \mathrm{pF}$ decoupling capacitors and eight $10 \mathrm{M} \Omega$ resistors through which the High Voltage is applied to the wires. Chambers are lested? : gas leakage (less than $100 \mathrm{ml} / \mathrm{h}$ at $10 \mathrm{mbar}$ overpressure), wire strength (190-310 g) and dark current (less than $100 \mathrm{nA}$ at $3600 \mathrm{~V}$;.

The chambers use the same gas mixture as the hadron calorimeter barrel at about $1800 \mathrm{~V}$ to obtain $400 \mathrm{fC}$ for minimum ionizing particles. The 8064 channels readout system is the same as that of the barrel hadron calorimeter. For three central layers of each octant we also digitize the charge pulse from the other end of the chamber so that the coordinate along the wire can be obtained by charge partition. By cor bining the measurements on the thre layers, the precision on this coordinate ranges from $0.8 \%(3.1 \mathrm{~cm})$ to $1.2 \%(4.7 \mathrm{~cm})$ for a gate going from $250 \mathrm{~ns}$ to $400 \mathrm{~ns}$ [20].

The muon filter chambers were tested both in laboratory, with cosmic rays, and with the $X 3$ beam at the CERN SPS [21,22]. In the laboratory tests, several $\mathrm{Ar}-\mathrm{CO}_{2}$ mixtures were used. Figs. 77 and 78 show some results that demonstrate the very saiisfactory chamber behavior. The overall chamber efficiency, measured during the tests on the SPS test beam, was $91 \%$, giving $97 \%$ 


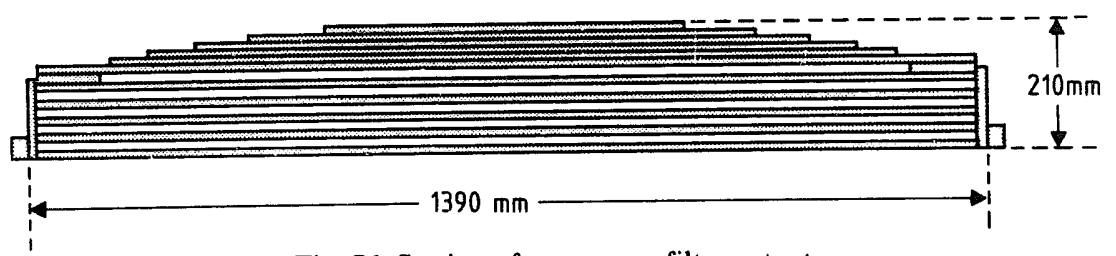

Fig. 76. Section of one muon filter octant.

when unfolded for geometrical effects (wall thickness, plastic supports).

\section{Scintillation counters}

The good time resolution ( $<1 \mathrm{~ns}$ ) of the scintillation counters will be used to distinguish di-muon events from cosmic muons. A single ' osmic muon which passes near the interaction point resembles a muon pair event producto in $\mathrm{e}^{+} \mathrm{e}$ interaction, but the time-of-flight difference between opposite scintillation counters is $\mathbf{5 . 8}$ ns for cosmic muons and zero for muon pairs.

\subsection{Counter dimensions}

The scintillation counters are located between the electromagnetic and hadronic calorimeters (fig. 79). In this position nearly complete coverage of the solid angle

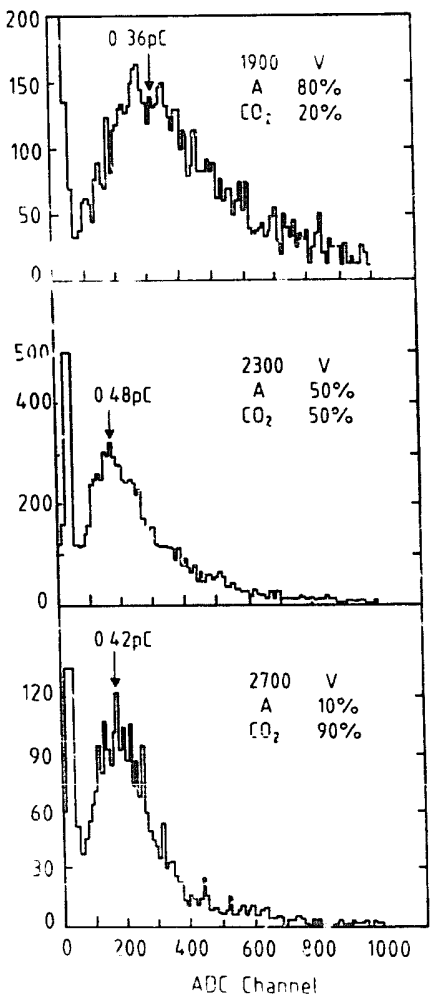

Fig. 77. Typical charge distribution obtained with different $\mathrm{Ar}+\mathrm{CO}_{2}$ mixtures. can be achieved and the scintillator can be used in the trigger on hadronic events. The barrel scintillation counters are bent to follow the shape of the hadron calorimeter barrel: they are $875 \mathrm{~mm}$ away from the beam at the position of the hadron calorimeter rings R0, R1 and at $969 \mathrm{~mm}$ at the position of rings R2, R3, R4. We use a $1-\mathrm{cm}$ thick Bicron BC-412 plastic scintillator. The counter is $167 \mathrm{~mm}$ wide in the middle and 182 $\mathrm{mm}$ at the ends in order to cover the same solid angle for the inner and outer rings of the hadron calorimeter. The projected length of the scintillator is $2900 \mathrm{~mm}$. We use adiabatic light guides made of UV transparent Plexiglas GS218 (Roehm, FRG). A barrel counter has a $320-\mathrm{mm}$ long light guide on either end. The counters are grouped in 16 pairs. Each pair covers one hadron calorimeter $\Phi$ sector. Both $\Phi$ sectors near the rails for the BGO calorimeter are covered only by two wider counters. Thus in total there are 30 barrel counters. The light guides are asymmetric so that the phototubes of two adjacent counters are close to each other. In this way there is enough space for cables between pairs of phototubes.

The end cap counters are located in front of the end cap hadron calorimeter (fig. 80). The light guides have a $90^{\circ}$ bend and extend to the end of the hadron calorimeter end caps. There are 16 counters on either side of the detector each one viewed by a single tube. The scintillator is $270 \mathrm{~mm}$ long, $10 \mathrm{~mm}$ thick, $275 \mathrm{~mm}$ wide on one side and $180 \mathrm{~mm}$ on the other. It is perpendicu-

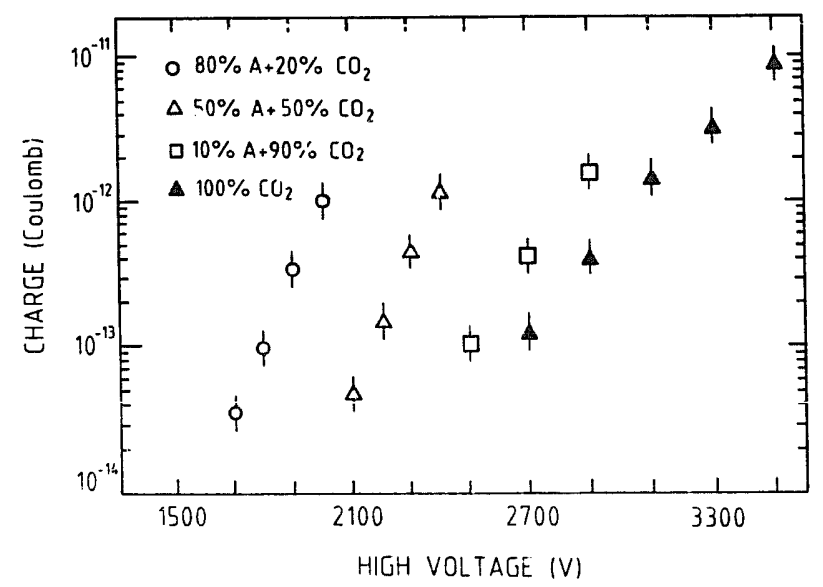

Fig. 78. Collected charge $\mathrm{fr}$ - minimum ionizing particles as a function of the applied high voltage for dirterent $\mathrm{Ar}+\mathrm{CO}_{2}$ mixtures. 


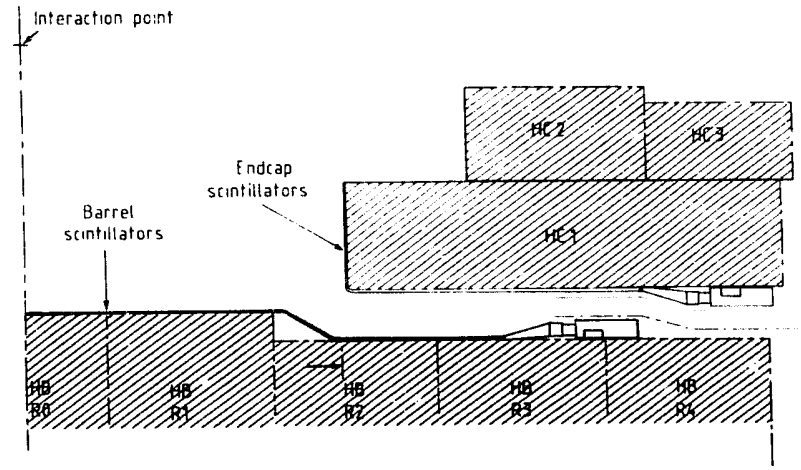

Fig. 79. Cut through the barrel and end cap hadron calorimeter indicating the location of the scintillation counter. The barrel counter is viewed by a phototube on each side (only half of the counter is shown).

lar to the beam line, $1030 \mathrm{~mm}$ from the interaction point. Each counter covers one of the $16 \Phi$ sectors of the hadron calorimeter endcaps.

The barrel counters angular coverage is $|\cos \theta|<$ $0.83\left(34^{\circ}<\Theta<146^{\circ}\right)$, where $\Theta$ is the polar angle with respect to the beam line. They cover therefore the acceptance of the middle muon chamber (MM). The end cap counters extend the coverage down to $\mid \cos$ $\Theta \mid<0.90\left(25^{\circ}<\Theta<155^{\circ}\right)$. In the azimuthal angle $\Phi$, $93 \%$ of the solid angle is covered by scintillators.

\subsection{Photomultiplier}

The Hamamatsu R2490-01 16 stages mesh dynode photomultiplier operates in the 0.5-T field region with high quantum efficiency $(14 \%$ at $430 \mathrm{~nm}$, the maximum in the emission spectrum of the BC-412 scintillator), high gain $\left(4 \times 10^{5}\right.$ at $0.5 \mathrm{~T}$ magnetic field compared to $1.5 \times 10^{6}$ without magnetic field) and good time resolution. The rise time of the anode pulse is $2.7 \mathrm{~ns}$ with a

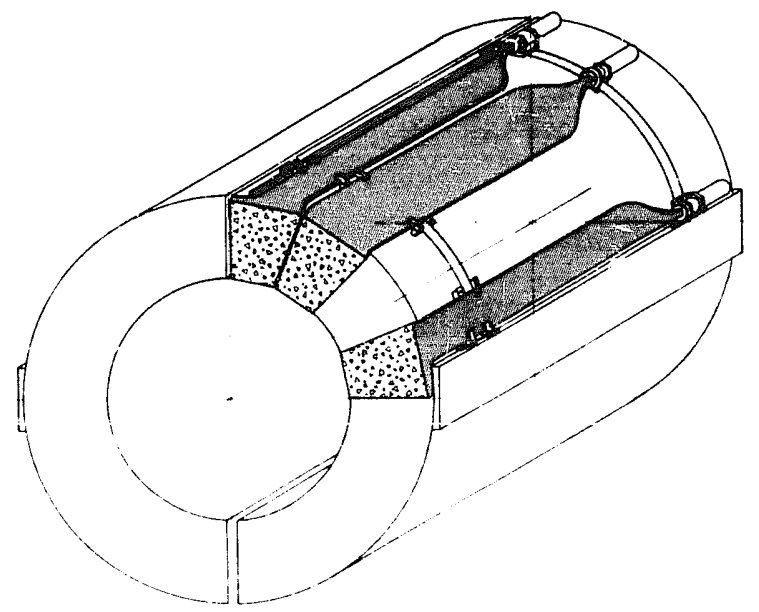

Fig. 80. The end cap scintillators are mounted on the end cap hadron calorimeter $\mathrm{HCl}$. transit time jitter of $0.9 \mathrm{~ns}$ for one photoelectron and $0.6 \mathrm{~ns}$ for 0 photoelectrons.

\subsection{Readour}

The 92 signals are digitized in LeCroy FASTBUS TDC 1875, with a resolution of $50 \mathrm{ps}$ and a dynamic range of 15 bits. The time resolution of the counters can be improved by a factor of two because we also digitize the charge of the pulse, so that time slewing corrections can be applied. A paddle card has been built to convert the photomultiplier pulse to the differential signals needed for the LeCroy 1885 ADC.

\subsection{Test results}

Acceptance tests have been performed with cosmic rays on all counters, with the following results:

The mean attenuation length is $1.7 \pm 0.2 \mathrm{~m}$. A cosmic ray which penetrates the middle of the counter yields on average 41 photoelectrons. A time resolution of $0.35 \mathrm{~ns}$ has been measured for the mean time of both phototubes. We have also tested a counter with phototubes in a magnetic field of $0.5 \mathrm{~T}$. The number of photoelectrons is not affected by the magnetic field, but the gain is reduced by a factor of 2.2 compared to zero field. Similar results have been obtained for the end cap counters. Here we have measured 80 photoelectrons for a minimum ionizing particle penetrating the scintillator.

\section{The electromagnetic detector}

The electromagnetic detector has excellent energy and spatial resolution for photons and electrons over a wide energy range (from $100 \mathrm{MeV}$ to $100 \mathrm{GeV}$ ). It uses bismuth germanate (BGO) as both the showering and detecting medium. BGO is a particularly attractive material for an electromagnetic calorimeter because it has hig,l stopping power (short radiation length) for photons and electrons, and large nuclear interaction length. Furthermore, it has low afterglow and is not hygroscopic.

The electromagnetic calorimeter [23] consists of about 11000 BGO crystals pointing to the iriteraction region. Each crystal is $24 \mathrm{~cm}$ long and is a truncated pyramid about $2 \times 2 \mathrm{~cm}^{2}$ at the inner end and $3 \times 3 \mathrm{~cm}^{2}$ at the outer end. Two silicon photoudiodes and associated linear electronics detect the light. The energy resolution is $\approx 5 \%$ a i $109 \mathrm{MeV}$ and below $1 \%$ for ericrgies above $\approx 2 \mathrm{GeV}$; the measured spatial resolution above $2 \mathrm{GeV}$ is better than $2 \mathrm{~mm}$ and the hadron/electron rejection ratio about 1000:1. The detector (fig. 81) surrounds the vertex chamber and consists of: 


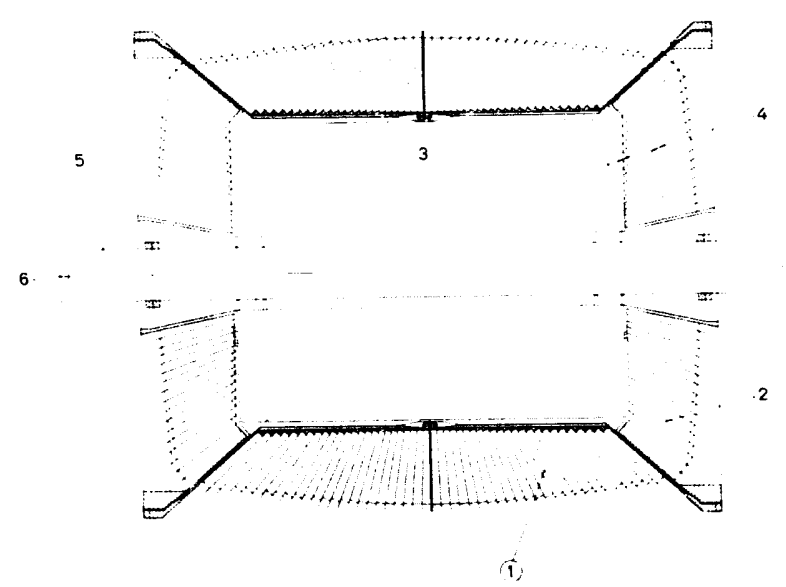

Fig. 81. Longitudinal cut through the BGO detector.

i) two half-barrels (EB) made of BGO crystals;

ii) two end caps (EC) made of BGO crystals, with track chambers (FTC) in front (to be instilled in Phase II).

\subsection{The barrel}

The 7680 crystals of the barrel (table 8) are arranged in two symmetrical half-barrels, giving a polar angle coverage $42^{\circ}<\Theta<138^{\circ}$.

\subsubsection{BGO crystals}

The BGO crystals (table 9) are produced by the Shanghai Institute of Ceramics in China, using a modified Bridgeman method. Very pure $\mathrm{Bi}_{2} \mathrm{O}_{3}$ and $\mathrm{GeO}_{2}$ powders (impurities $<10^{-6}$ ) are mixed in the correct stoichiometric proportions. The resulting polycrystalline powder of $\mathrm{Bi}_{4} \mathrm{Ge}_{3} \mathrm{O}_{12}$, contained in a platinum foil crucible, is introduced into an oven. Then a temperature gradient is slowly displaced relative to the melt, starting from a BGO monocrystal seed. The crucible has the shape of the final crystal but is somewhat larger to allow for later machining.

\section{Table 8}

Main parameters of the BGO barrel

\begin{tabular}{ll}
\hline Inside radius of barrel & $52 \mathrm{~cm}$ \\
Inside length of cylinder & $100 \mathrm{~cm}$ \\
Material in front of crystals & $0.05-0.1 X_{0}$ \\
Angular coverage & $42.3^{\circ}-137.7^{\circ}$ \\
Number of crystais & $2 \times 3840$ \\
Number of crystal types in $\Theta$ & 24 \\
$\begin{array}{l}\text { Number of crystals per type in } \Phi \\
\text { Crystal dimensions }\end{array}$ & 160 \\
$\quad$ & $24.0 \mathrm{~cm}$ \\
$\quad$ length & $2 \times 2 \mathrm{~cm}^{2}$ \\
$\quad$ ront face & $2.6 \times 2.6$ to $2.9 \times 2.9 \mathrm{~cm}^{2}$ \\
$\quad$ volume & $130-150 \mathrm{~cm}^{3}$ \\
\hline
\end{tabular}

Table 9

BGO and $\mathrm{NaI}(\mathrm{Tl})$ properties

\begin{tabular}{|c|c|c|}
\hline & BGO & $\mathrm{NaI}(\mathrm{Tl})$ \\
\hline Density $\left[\mathrm{g} / \mathrm{cm}^{3}\right]$ & 7.13 & 3.67 \\
\hline Radiation length $[\mathrm{cm}]$ & 1.12 & 2.59 \\
\hline Moliere radius [cm] & 2.3 & 4.4 \\
\hline $\mathrm{d} E / \mathrm{d} x[\mathrm{MeV} / \mathrm{cm}]$ & 9 & 4.8 \\
\hline Interaction length [cm] & 22 & 41 \\
\hline Refractive index & 2.15 & 1.85 \\
\hline $\begin{array}{l}\text { Waveitngth of maximum } \\
\text { emission [nm] }\end{array}$ & 480 & 410 \\
\hline Relative light output & $8-15$ & 100 \\
\hline $\begin{array}{l}\text { Temperature coefficient } \\
\text { of light yield } \\
{\left[\% /{ }^{\circ} \mathrm{C} \text { at } 25^{\circ} \mathrm{C}\right]}\end{array}$ & -1.55 & 0.22 \\
\hline $\begin{array}{l}\text { Lumin. lifetime at room } \\
\text { temperature }[\mu \mathrm{s}]\end{array}$ & 0.3 & 0.23 \\
\hline $\begin{array}{l}\text { Afterglow at } 3 \mathrm{~ms} \\
\text { Hygroscopic }\end{array}$ & $\begin{array}{l}0.005 \% \\
\text { no }\end{array}$ & $\begin{array}{l}0.5-5 \% \\
\text { yes }\end{array}$ \\
\hline
\end{tabular}

The produced ingots are then cut to size and polished. The tolerances in dimensions are dictated by the need to have a safe mounting in the support structure together with a minimum dead space between crystals:

- from $-300 \mu \mathrm{m}$ to $0 \mu \mathrm{m}$ in transverse dimensions,

- from $-400 \mu \mathrm{m}$ to $0 \mu \mathrm{m}$ in length,

- less than $50 \mu \mathrm{m}$ in planarity of all faces.

All crystals are truncated pyramids (fig. 82 and table 10) pointing to the interaction region, with a small angular offset $(10 \mathrm{mrad}$ in $\Phi)$ to suppress photon leakage. Their cross section is approximately square and they have a constant front face area. This geometry involves 24 types of crystals, with shape changing slowly along $\Theta$ due to the variable distance to the interaction point (fig. 81). f.n accurate $(\approx 50 \mu \mathrm{m})$, fast, cheap and safe method for cutting and polishing the crystals was developed [24], consisting in sawing directly the crystals to the required dimensions with a diamond disk. The surface finish obtained allows us to proceed directly to mechanical polishing of nine crystals simultaneously on a spinning table.

Systematic tests [25] were performed on all crystals arriving at CERN in monthly batches of 130 to 400 crystals during three years. Each crystal was first in-
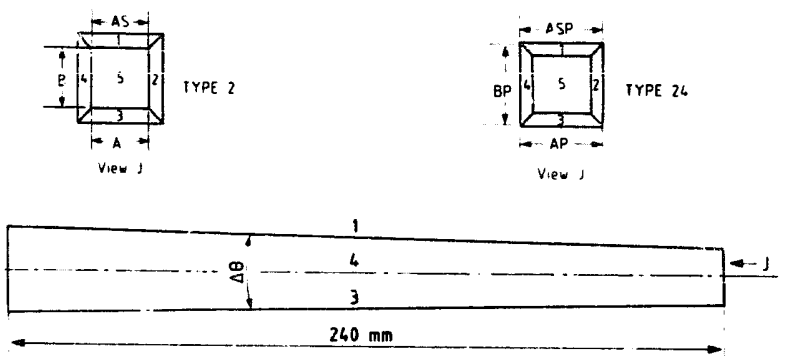

Fig. 82. Typical crystal shapes for the barrel. See also table 10. 
Table 10

Dimensions of a few representative crystals in the barrel. NCR is the crystal row number, $\theta$ (deg) its polar angle with. respect to the bean axis and $d \Theta$ its angular aperture. The meaning of the other quantities (in $\mathrm{mm}$ ) is shown in fig. 82.

\begin{tabular}{rllllllll}
\hline NCR & A & AS & B & AP & ASP & BP & $\Theta$ & d $\Theta$ \\
\hline 2 & 20.12 & 20.17 & 20.54 & 29.52 & 29.59 & 30.14 & 87.7040 & 2.2898 \\
12 & 20.12 & 20.46 & 20.39 & 28.57 & 29.05 & 28.95 & 65.6825 & 2.0434 \\
24 & 20.12 & 20.70 & 20.27 & 26.48 & 27.24 & 25.68 & 43.9097 & 1.5283 \\
\hline
\end{tabular}

spected by eye and any defect was recorded. Then, the optical quality of the crystals was checked by a measurement of the transparency spectrum on their full length [26]. Typical transmission curves are shown in fig. 83. The three reference points at 400,480 and 630 $\mathrm{nm}$ are minimum transparency values required in the specifications.

A departure from the transmission profiles shown in fig. 83, especially in the near UV range, may indicate a weaker resistance to radiation. This effect was observed on a few prototype crystals. During the quality control of the barrel crystals, several batches of a few crystals each were checked for radiation hardness. They were exposed to a dose of $10^{3} \mathrm{rad}$ from a medical ${ }^{60} \mathrm{Co} \gamma$ ray source. This is more than 100 times the daily dose expected for the barrel at LEP in the worst case scenario (beam loss, etc.). Immediately after irradiation, the transparency in the blue region had decreased by about $40 \%$. Then it was observed to recover spontaneously ai room temperature. Full recovery of the original transmission profile was reached after one month. It should also be stressed that the scintillation efficiency was not affected by the irradiation. This was demonstrated by comparing the light output of small and large crystals.

The cr.'stal dimensions, planarities and angles were checked on a measuring bench [27], simultaneously re-

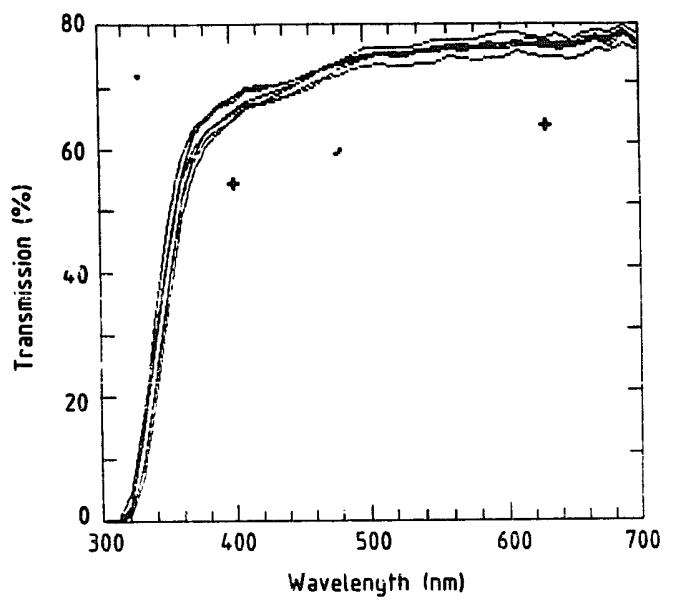

Fig. 83. Optical transmission of a few crystals through their full length as a function of the wavelength. The crosses represent the minimum transmission values accepted. cording all dimensions of a crystal by comparison with an accurate steel standard, with the help of 39 inductive position sensors. The accuracy of each measured point was better than $10 \mu \mathrm{m}$. After the initial batches, the crystal rejection rate for bad aspect (dents, scratches, etc.), low transparency or incorrect dimensions was $0.2 \%$.

The light collected at the large end face of a tapered crystal with its six faces polished increa es strongly with the distance from the light source to the large end face. This is shown in fig. 84 for a crystal viewed by a photomultiplier and illuminated by a ${ }^{137} \mathrm{Cs}$ source. Good linearity and energy resolution require a nearly uniform light collection efficiency, as indicated by test measurements and Monte Carlo simulation. By coating the polished crystals with a $40-$ to $50-\mu \mathrm{m}$ thick layer of high reflectivity NE560 paint, one obtains a nearly flat light collection efficiency curve (fig. 84), with a light output comparable to that reached with the best wrappings tested (160-270 $\mu \mathrm{m}$ thick).

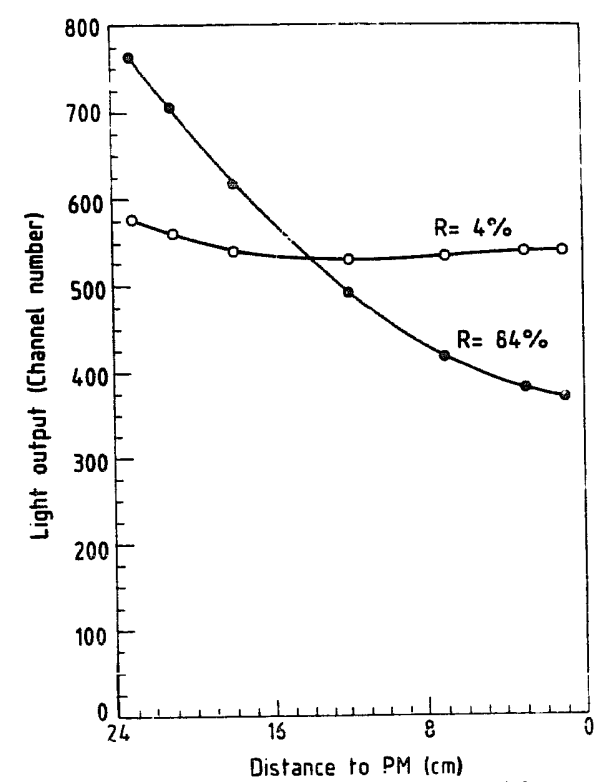

Fig. 84. Light collection curves measured with a collimated ${ }^{137} \mathrm{Cs}$ source running along the crystal main dimension. All crystal faces are polished. The parameter $R$ is the relative light output difference for the source at 21 and $3 \mathrm{~cm}$ from the photomultiplier. The dots correspond to aluminized mylar wrapping and the circles to white paint coating (see text). 


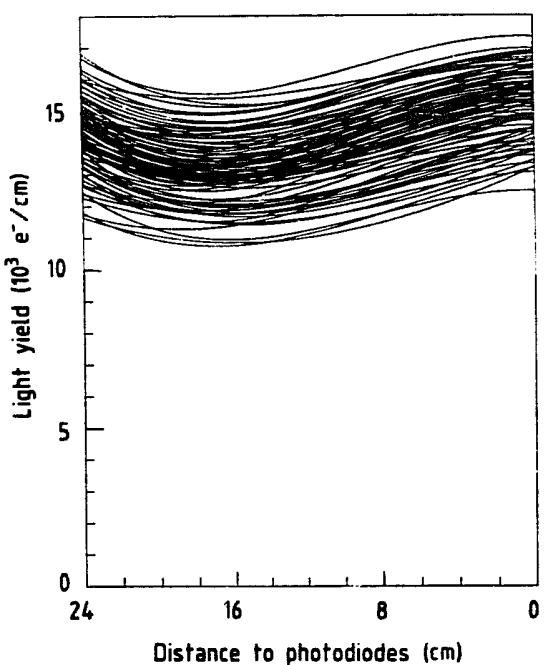

Fig. 85. Typical light collection curves obtained with the cosmic ray bench for a sample of 96 crystals. The light yields are measured by the number of photoelectrons per $\mathrm{cm}$ of ionized track.

To guarantee the performance of the crystals for energy measurement, it was necessary to check their light output and the uniformity of light collection. The light output is an important parameter for the resolution at low energy ( $100 \mathrm{MeV}$ to about $2 \mathrm{GeV}$ ), which is dominated by the signal-to-noise ratio. We used the ionization by cosmic muons for these measurements [28].

A test bench capable of simultaneous measurement of the response of 40 crystals per day and reconstruction of track length and position along the crystals was set up. The painted crystals were equipped with capsules holding two photodiodes (Hamamatsu S-2662, 1.5 $\mathrm{cri}^{2}$ active area), followed by preamplifier, shaping amplifier and ADC. The data obtained (fig. 85) can to considered as a precalibration of the crystals and were very useful for understanding the final beam calibrations of the two half-barrels. Fig. 86 shows the light yield versus the uniformity parameter $R$ defined as the relative variation of the collection efficiency of the light produced at the crystal extremities. These measurements were made with cosmic rays, for all crystals of the first half-barrel. Limits were set on these parameters to avoid low light output tails or light collection curves too far away from the optimum value. Crystals out of limits were corrected by paint additions.

\subsubsection{Mechanical structure}

The rucchanical structure of the barrel (figs. 87 and 88 ) bears the weight of the 7680 crystals, the preamplifier boards and the corresponding cables, monitoring devices and cooling circuitry. To achieve the best solid angle coverage and to minimize dead spaces between crystals, the structural material is confined to thin walls

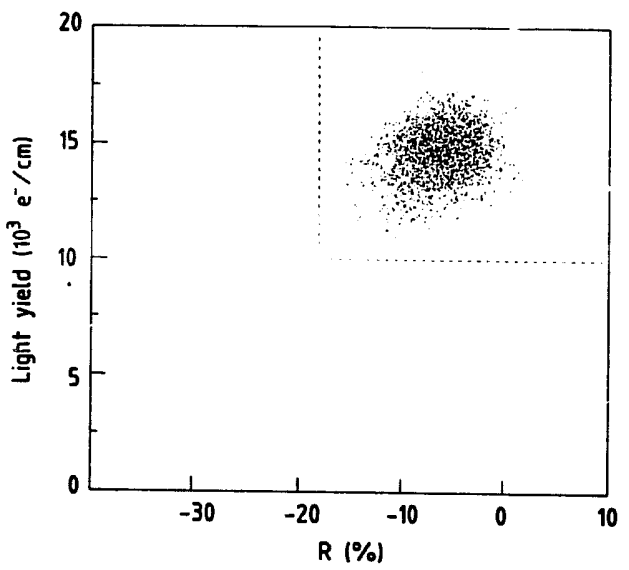

Fig. 86. Light output versus uniformity parameter $R$ for all the crystals of the first half-barrel. The average light output is $(14.4 \pm 3.8) \times 10^{3} \mathrm{e} / \mathrm{cm}$ and the average value of $R$ is $(-6.9 \pm$ $3.8) \%$. The best energy resolution is obtained for $R$ values between 0 and $-10 \%$.

around the cells and to a cylindrical inner tube attached on each side to a conical funnel ("trumpet") which carries the weight and transmits it to the four bearing pads. The whole structure was studied on a computer aided design system [29].

For the calibration of the calorimeter, it is necessary to aim an electron beam of known momentum at each crystal individually, with the beam passing through the geometrical center of the barrel. Therefore, the barrel is split into two halves along a plane normal to its axis. As a consequence, each half-barrel has to be reinforced at the cut with a $0.5-\mathrm{mm}$ austenitic steel membrane, which is mostly needed during the coupling operation of the two half-barrels and remains in place afterwards.

The cell walls, as well as the inner tube, are made of epoxy resin carbon fiber composite [30]. This material

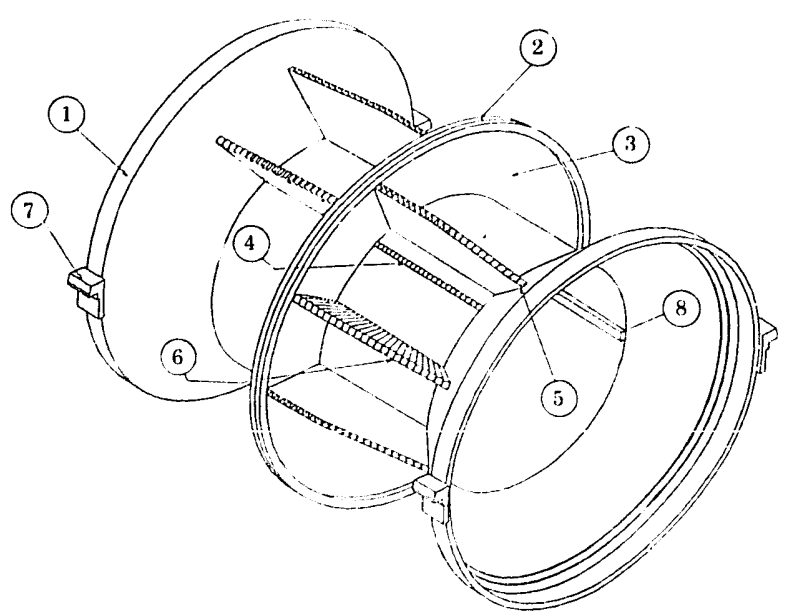

Fig. 87. Isometric view of the mechanical structure for the BGO barrel. (1) Trumpet. (2) Middle flange. (3) Middle membrane. (4) Barrette. (5) Slice. (6) Row of 24 crystals (7) Roller bearing. (8) TEC supporting rail. 


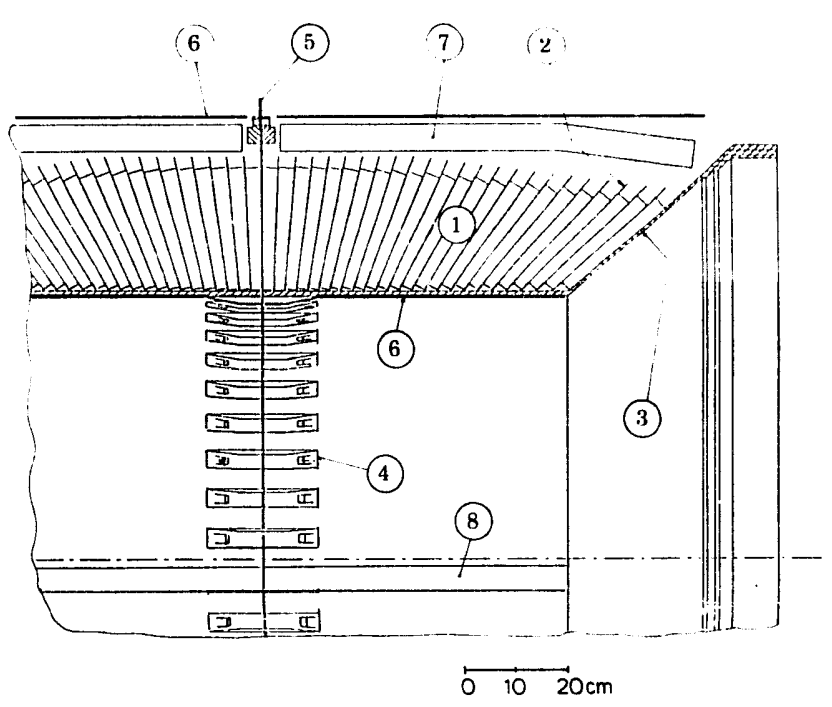

Fig. 88. Longitudinal section through the BGO barrel. (1) Crystals. (2) Cell walls (3) Trumpet body out of carbon fiber composite. (4) Reinforcement pads for titanium bolts. (5) Middle flange with stainless steel membrane. (6) Thermal shield.

(7) Preamplifier board. (8) TEC supporting rail.

offers excellent mechanical properties and is of relatively low density. The minimum value of the Young's modulus is $E=45000 \mathrm{~N} / \mathrm{mm}^{2}$ and the yield stress is $\sigma_{\mathrm{e}}=350 \mathrm{~N} / \mathrm{mm}^{2}$. The tube thickness corresponds to 0.04 radiation length at normal incidence. Moreover, this composite material has good molding properties and is very suitable for building a complex structure with the required precision. Metallic parts are introduced in some places for very specific purposes:

- Titanium alloy screws to connect the two half-barrel structures (low density, high resistance alloy ASTM 3.7164).

- High resistance aluminum alloy (Anticorodal 100) pads to connect the supporting structure to the four roller bearings behind the crystals.

BGO crystals are extremely sensitive to permanent loading, with risks of subcritical crack propagation [31]. Each crystal is thus held in a separate cell with clearances such that normal structural deformation does not affect any crystal and that the weight of any crystal is not transferred to its neighbors. A total of 160 modular molded "slices" with 24 cells each are glued side by side on the supporting structure in order to produce a complete half barrel. A step-shaped part, called a "barrette", closes the slice bottom and provides accurate longitudinal crystal position.

Each crystal is pressed from its back onto the front of its cell by a spring-loaded device which pulls on the walls with a force of about $20 \mathrm{~N}$ (crystal weight $\approx 1 \mathrm{~kg}$ ) ensuring that a positive force is exerted on the bottom of the cell in any position and that the crystal cannot slide sideways nor press on the cell wall.
The thickness of the cellular structure has been minimized to the limit of technical feasibility: each crystal is separated from its neighbors by a composite wall, made of two layers of $100 \mu \mathrm{m}$ pre-impregnated carbon cloth. The final thickness varies between $200 \mu \mathrm{m}$ and $250 \mu \mathrm{m}$. A nominal clearance of $100 \mu \mathrm{m}$ is kept between the crystal faces and the walls in the $\bar{\Phi}$ and $\Theta$ directions. It allows for the paint coating and the expected elastic deformation of the structure due to the changes in position of the detector, from assembly to calibration and to experiment, without stressing the crystals. Cellular walls and clearances represent about $1.75 \%$ of the solid angle covered by the barrel.

The supporting structure is made of two symmetrical shells connected by titanium bolts in the mididle plane. Each shell is a composite trumpet, 1-m long object with a 10-mm thick cylindrical part in front of the crystals and a 5-mm thick conical part along the side of the last barrel crystals at $\Theta=47^{\circ}$. A $25-\mathrm{mm}$ thick solid ring with fixtures for the bearings reinforces the cone edge. The cylinder inside diameter is $1015 \mathrm{mrn}$; the solid ring outside diameter is $1600 \mathrm{~mm}$. Individual crystal weights and pulling resultants are gathered on the trumpet cylindrical shell and transmitted to the bearings through the conical shell and the solid ring. The maximum trumpet deformation, calculated [32] and measured during a preassembly loading, is less than $1 \mathrm{~mm}$ including bending and section deformation. This deformation is uniformly shared on to the cellular structure. The composite structure weighs $140 \mathrm{~kg}$ for a total load of $10 \mathrm{t}$.

\subsubsection{Electronics}

Since the BGO calorimeter is operating in a $0.5-\mathrm{T}$ magnetic field and space is at a premium, conventional photomultipliers cannot be used. Instead, we use 1.5 $\mathrm{cm}^{2}$ Hamamatsu S2662 photodiodes to detect the BGO scintillation light; they are insensitive to the magnetic field and have a quantum efficiency of about $70 \%$. Each crystal has two photodiodes glued to its rear face. The total diode capacitance is $230 \mathrm{p} \bar{\Gamma}$ for $15 \mathrm{~V}$ reverse bias. Since they have unity gain (each photon detected produces one electron-hole pair), a preamplifier must be added. The signal from the photodiode is about $0.2 \mathrm{fC}$ (1200 electrons) for each MeV deposited in the BGO [33].

The charge sensitive preamplifier [34] is mounted directly behind the crystal and uses a low noise, high transconductance Toshiba 2SK147 FET in a cascode configuration. The output pulse rise time is $300 \mathrm{~ns}$ (corresponding to the BGO light decay time) and the exponential decay time is $800 \mu \mathrm{s}$. The rms random noise level of the photodiode and preamplifier combination is less than 1000 elect.ons. This is much higher than the value that can be obtained with photomultipliers, but significantly affects the resoluticn only for very small signals (showers less than a few hundred $\mathrm{MeV}$ ). The 
amplifier gain is extremely stable however and we do not expect any of the gain variations that are normally experienced when using photomultipliers. A test pulse input to the preamplifier is provided.

The analog to digital converter (ADC) units, one for each crystal (fig. 89) are mounted $3 \mathrm{~m}$ away, just outside the hadron calorimeter. The ADC has been designed to satisfy two basic requirements: to measure signals accurately over a wide dynamic range, from $100 \mathrm{MeV}$ to 100 $\mathrm{GeV}$, and to have a short memory time so that the tails from large signals do not mimic small signals in later beam crossings. The signal from the preamplifier is differentiated with a pole zero circuit which replaces the $800 \mu \mathrm{s}$ exponential decay with a $1.1 \mu \mathrm{s}$ decay time, and split three ways, into a programmable attenuator channel for the trigger (see section 12), and into two separate ADC channels, one for small signals and one for large signals. Each channel has its own independent resettable integrator and a sample-hold circuit.

The low range has an additional gain of 32 before the integrator. After each beam crossing, the signal is integrated and stored by the sample-hold circuit. Then the integrator is reset, in preparation for the next beam crossing. This provides the short integrator memory time for large signals. The sample-hold (with the stored signal) is not released until just before the next beam crossing, allowing maximum time for the first level trigger to operate. The sample-hold circuits are followed by two amplifiers, each providing a gain of four to yield the desired wide dynamic range. A single chip microcomputer with its program in masked ROM (Hitachi 6305) chooses one of the six signals available to digitize using a 12-bit digital to analog converter (DAC) and six comparators. A simple successive approximation algorithm is used on the chosen signal. The least count on the most sensitive range (the low energy channel and a gain of 16 after the sample-hold) is $5 \mu \mathrm{V}$, corresponding to less than $100 \mathrm{eV}$. Full scale (on the least sensitive range, no gain after the preamplifier) is $10 \mathrm{~V}$, about $200 \mathrm{GeV}$. The digitizing range of the ADC is equivalent to a 21-bit ADC, with resolution of at least 10 bits $(1: 1000)$ for signals greater than $100 \mathrm{MeV}$. The linearity is better than $1 \%$ over the full range. The actual dynamic range achieved for BGO signals is $200000: 1$, from full scale to the noise level.

The microcomputers (level 1 readout) complete the digitizing and store the data within $250 \mu \mathrm{s}$ after the trigger. The microcomputers, one for each BGO crystal, are organized in token ring networks of 60 crystals and are controlled by another computer (level 2 readout), which is a single board Motorola 68010 in a VME crate. These are located more than $100 \mathrm{~m}$ away in the counting room. Communication is via differential TTL drivers and receivers. Only the actual analog to digital conversion is done at the trigger time; readout by the higher level computers is done as a background task. The microcomputers can buffer up to 41 events internally. This system allows a peak instantaneous event rate of 4000 triggers per second and an average rate of 500 triggers per second (the transfer rate to the master). The microcomputers also allow several system features to be added at the lowest level. Each crystal has an individual sparse scanning threshold, trigger attenuator constant (for the analog output to the trigger system), trim

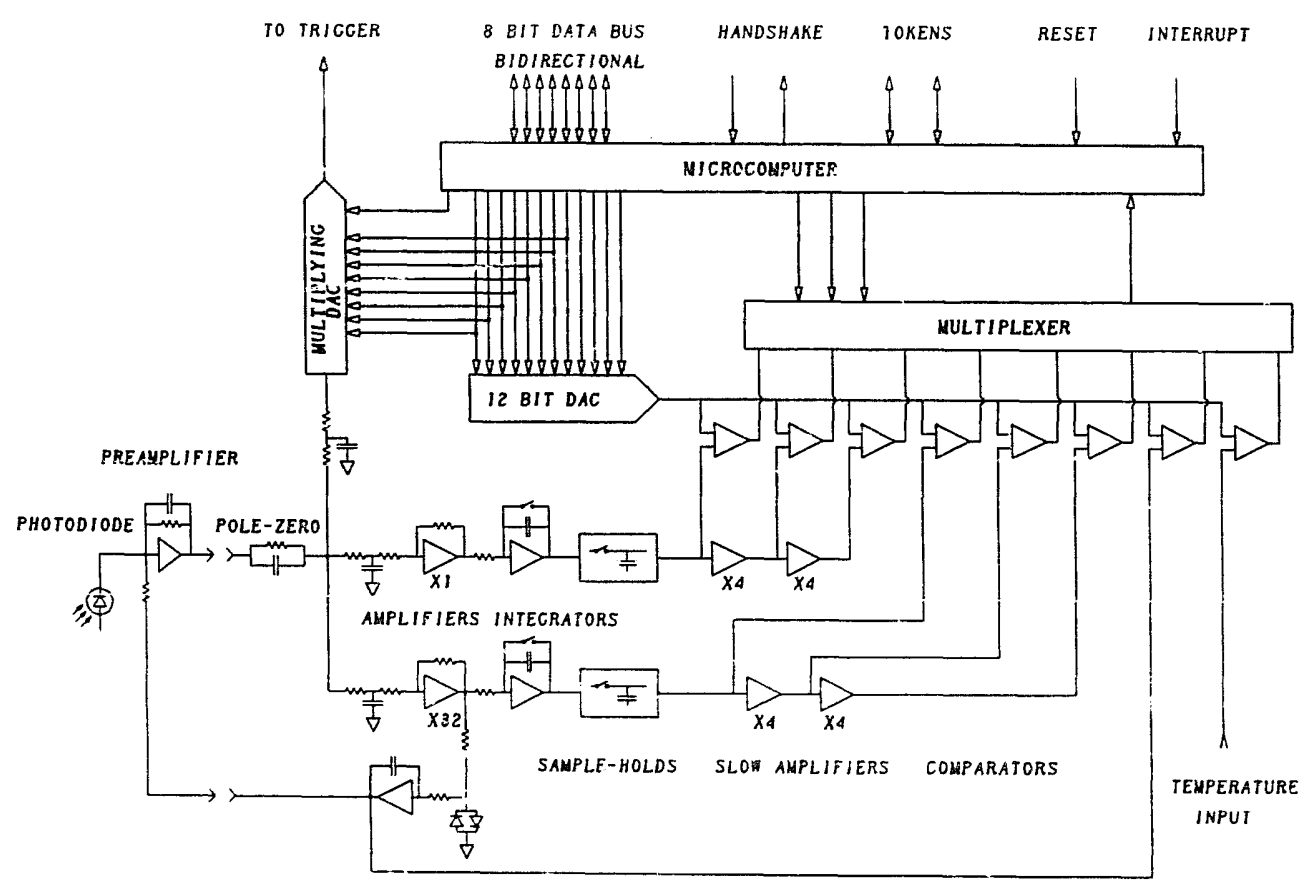

Fig. 89. Diagram of the level $1 \mathrm{ADC}$ system. 


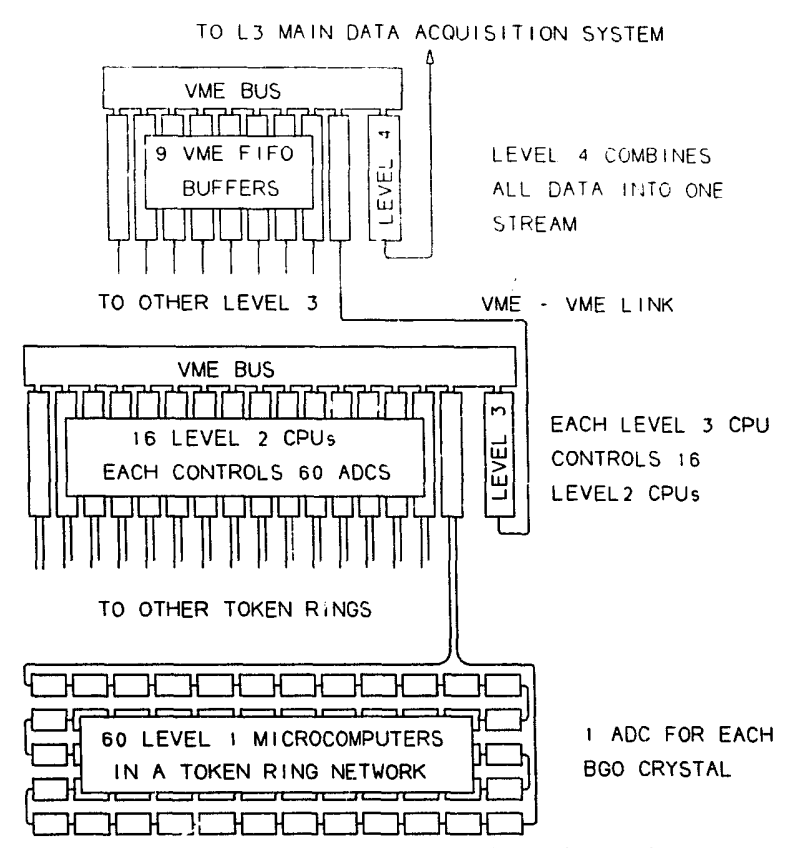

Fig. 90. Organization of the upper levels of the readout system.

constant (to adjust the pedestal value), test pulse enable, etc.; all of which are downloaded from the level 2 computer. Each ADC cais also measure the leakage current of the photodiodes al d, if desired, the temperature of the BGO crystal. Seviral test modes are available to ensure the integrity of the system. All the data words have a parity bit added before they leave the level 1 microcomputer. Fig. 90 shows the organization of the upper levels.

The level 2 computcrs are organized in groups of 16 and controlled by a level 3 computer (also Motorola 68010), which is the VME crate master (the level 2 computers are VME slaves). Communication between level 2 and level 3 is via dual ported memory located in each level 2 computer. The programs in these computers are stored in RAM and are loaded during an initialization phase, either by downloading from a higher level, or from local non-volatile memory in each VME crate. Each VME crate provides readout and control for 960 crystals. Eight crates are required for the barrel, thirteen crates for the full calorimeter (with endcaps and including the luminosity monitor). These crates are connected to a level 4 crate via a VME to VME link, which incorporates FIFO memory buffers. The level 4 computer combines the data for each event into one block and sends it to a FASTBUS memory module in the main data acquisition system. The system has a maximum average data transfer rate greater than $6 \mathrm{Mbyte} / \mathrm{s}$. This corresponds to 500 triggers per second, with $20 \%$ of the crystals having data above the sparse scan threshold.

\subsubsection{Thermal regulation}

The light produced in a BGO crystal by a particle of a given energy is strongly correlated to the crystal temperature. The light output variation is $-1.55 \% /{ }^{\circ} \mathrm{C}$. Therefore we must maintain the BGO at the lowest possible temperature but above the dew point. The required energy resolution $(<1 \%$ at $50 \mathrm{GeV}$ ) implies maintaining the crystal temperature constant within a few tenths of a degree, as well as the temperature difference between the two crystal end faces below $0.5^{\circ} \mathrm{C}$.

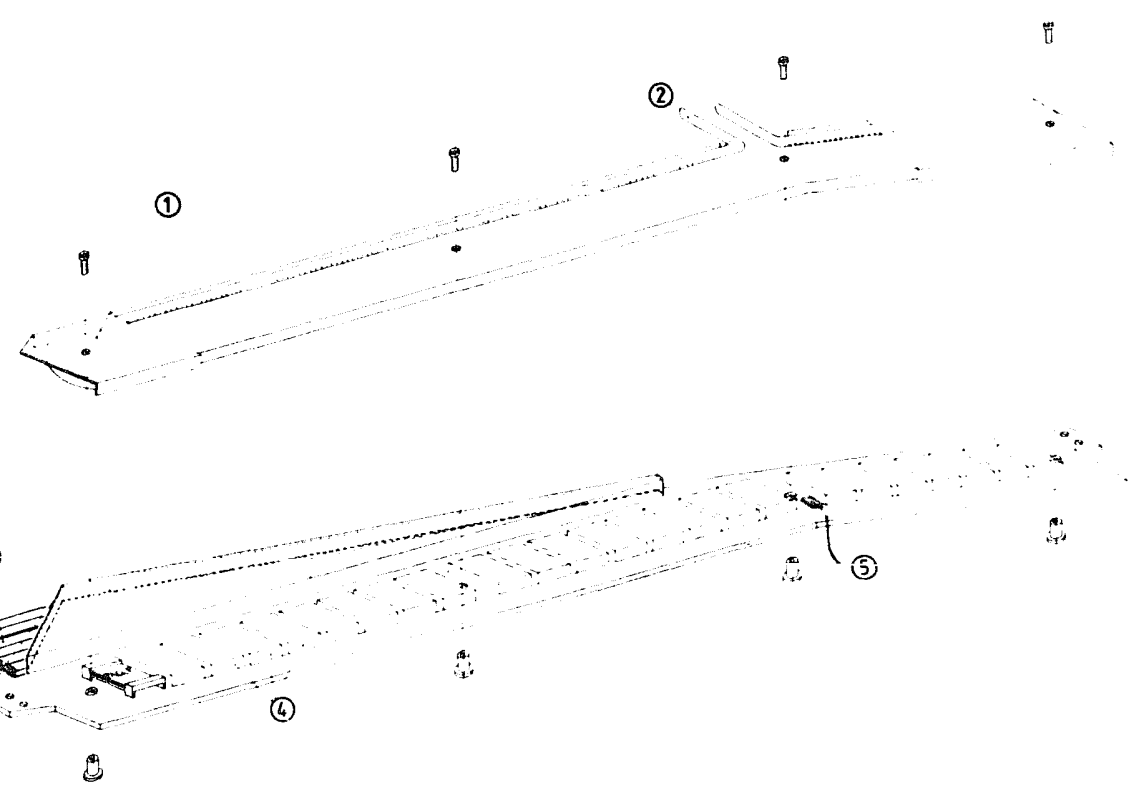

Fig. 91. Exploded view of a preamplifier board for the BGO barrei. (1) Thermal stabilization cover. (2) Cooling pipe. (3) Cables to the level 1 curd. (4) Preamplifiers. (5) Thermal sersors. 

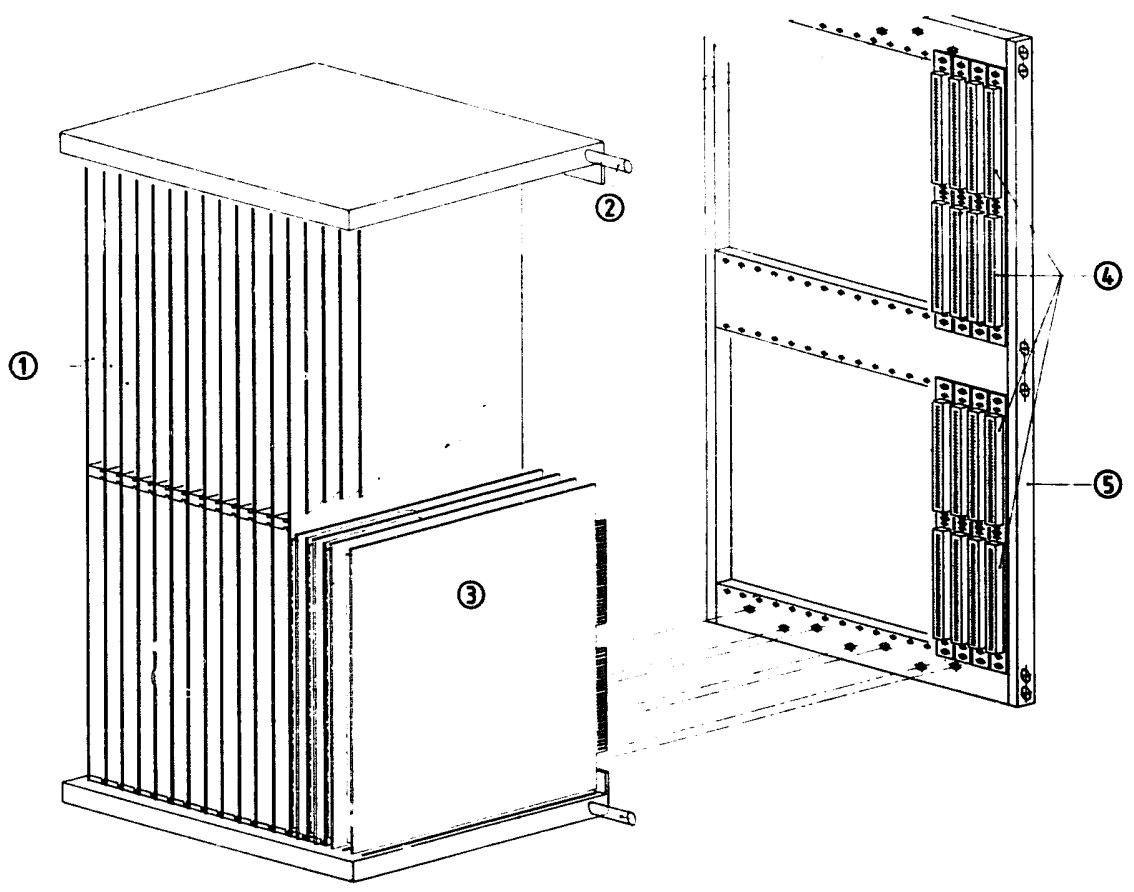

Fig. 92. Schematic view of a level 1 readout box. (1) Thermal screen. (2) Cooling pipe. (3) Level 1 readout board. (4) Connectors for the readout cables from the preamplifier boards. (5) Support frame for the readout box and for the cables.

The preamplifiers located at the rear face of the crystals dissipate about $0.2 \mathrm{~W}$ each. This heat must be removed to maintain the preamplifier temperature below $35^{\circ} \mathrm{C}$ and to minimize the gradients along the crystals. To evacuate this heat, each of the 320 24-channel boards is covered with a brass screen to which copper pipes have been soldered. The pipes are connected to cooling fluid circuits [35]. These screens are positioned about $1 \mathrm{~mm}$ away from the electronics components to achieve adequate heat transfer. A schematic layout of the preamplifier board and its thermal screen is shown in fig. 91. To prevent heat transfer to the electromagnetic calorimeter from outside, the calorimeter is surrounded by very thin active thermal shields, connected to the fluid circuits.

The level 1 readout boxes, each containing up to 32 readout electronics cards with 12 channels per card, are installed on the end faces of the hadronic calorimeter barrel. Each channel dissipates $2 \mathrm{~W}$, resulting in a total dissipation of $23 \mathrm{~kW}$. This heat has to be evacuated to maintain the electronics below $40^{\circ} \mathrm{C}$. A level 1 readout box is equipped with 17 built-in cooling screens, sandwiched with readout cards. Special screens were developed using the roll-bond technique [36]. Four boxes are connected to a cooling fluid circuit, resulting in a total of two sets of four circuits. A sciematic view of a cooling box is given in fig. 92 .

A s ooing fluid circuit [35] is basically composed of a vacuur fump, a circulation pump, a water-cooled heat exchanger and a thermal valve to regulate the fluid temperature (fig. 93). To avoid accidental fluid leaks, the circuit is operated at 800 mbar below atmospheric pressure. The cooling fluid must have low density, low viscosity, low vapor pressure and high specific heat; it must be an insulator to avoid mixing up the electrical

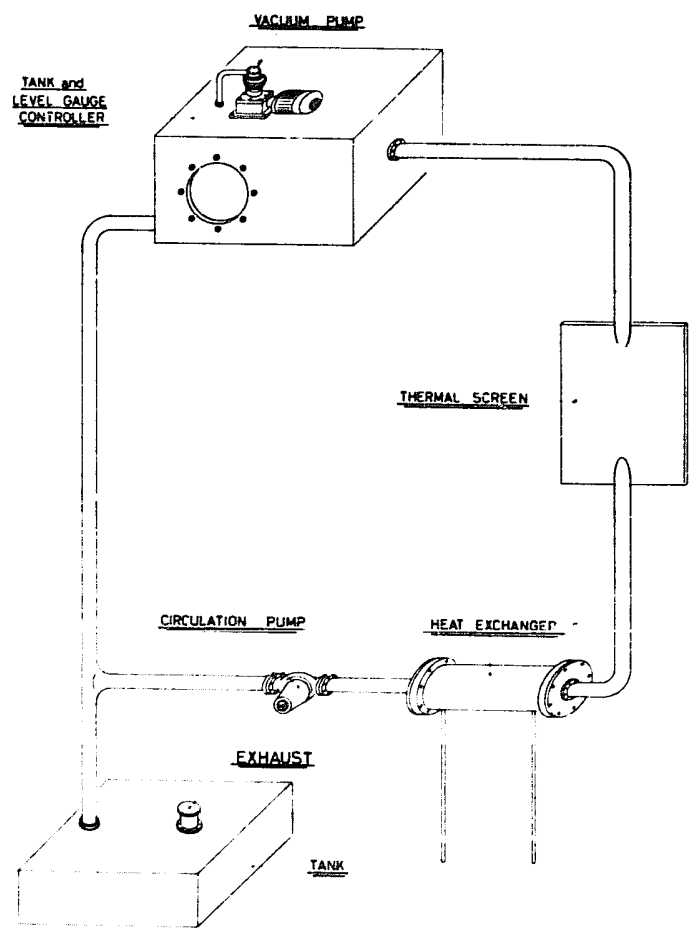

Fig. 93. Schematic view of a cooling circuit. 


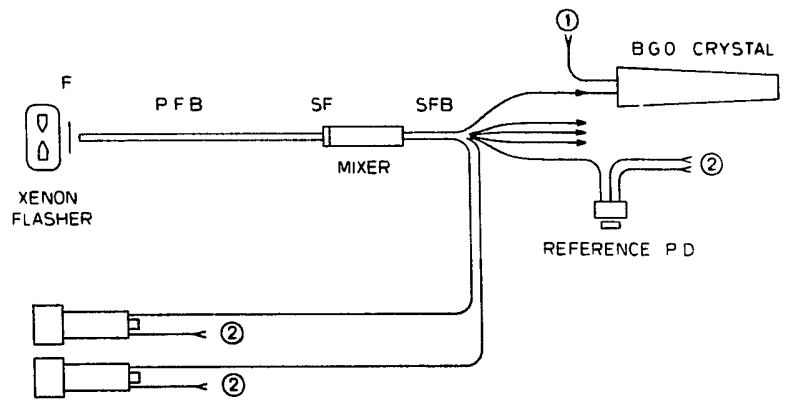

REFERENCE PM

Fig. 94. Schematic of the BGO xenon monitoring system. PFB: primary fiber bundle. SFB: secondary fiber bundle. SF: spectral filter. F: attenuation filter (optional). (1) Fiber from the other lamp system. (2) Fibers from other mixers.

grounds of the system. We use about $1 \mathrm{t}$ of a silicon-base liquid, Dow Corning DC $200 / 5 \mathrm{C}$ with a density of 1 $\mathrm{g} / \mathrm{cm}^{3}$, a viscosity of $5 \mathrm{cst}$ at $25^{\circ} \mathrm{C}$, a vapor pressure of $40 \mathrm{mbar}$ and a specific heat of $1400 \mathrm{~J} / \mathrm{kg}$.

The temperature at the front and back of the BGO crystals is monitored by 1280 AD590 sensors, so that there is one front and back temperature measurement for every 12th crystal. These sensors are read out through the BGO level 1 readout system. Another set of 960 AD590 sensors monitors the temperature of the preamplifier boards, of the level 1 readout boards and of the cooling fluids. They are read out by an independent system to permit safe operation of the electronics even in case of failure of the central BGO computer. The reading accuracy is about $0.1^{\circ} \mathrm{C}$ for each sensor.

\subsubsection{Xenon light monitor}

The light collection efficiency of each crystal Jgether with the gain of the corresponding readout chr in are monitored by means of xenon light pulses distributed by optical fibers (fig. 94). The light flashes are generated by a set of 16 xc ion flash lamps. From each lamp, the light is transporied by four bundles of optical fibers ("primary bu.ddles") to four light mixers and then to the crystals by four "secondary bundles" of 240 fibers each. From each mixer, additional fibers carry light to reference photomultipliers and photodiodes. Each crystal is illuminated by two fibers from two independent systems, one for high energy pulses (typically $35 \mathrm{GeV}$ equivalent), the other for low energy (about $1.5 \mathrm{GeV}$ equivalent). These two pulses are used to compare the behavior of the low and high gain channels of the BGO readout electronics. Furthermore, the sectors of the barrel covered by neighboring low and high energy fiber bundles are slightly offset. This enables the investigation of systematic effects.

The driving circuits of the Hamamatsu L2453 xanon lamps have been tuned to produce the same pulse length as the BGO scintillation. The primary bundles are made of 14 quartz fibers (type PCS600, core diameter $600 \mu \mathrm{m}$, produced by Fibres Optiques Industrie) which at each end are glued together in a connector. The length of a bundle is $35 \mathrm{~m}$. The light mixer consists of a polished lucite rod, $200 \mathrm{~mm}$ long, with a $5 \times 5 \mathrm{~mm}^{2}$ section. Upstream and downstream of this rod, slightly depolished lucite plates improve the mixing. In order to match the spectrum of the BGO scintillation light, the ultraviolet and infrared components are suppressed by appropriate filters. The secondary bundles, typically 2 $\mathrm{m}$ long, are composed of $200 / 230 \mu \mathrm{m}$ quartz/hard plastic fibers type HCP200 produced by Ensign Bickford Optics Company. At one end, the fibers are glued together in a connector and at the other end each fiber is terminated by a brass ferrule which snaps into the crystal capsule. The mechanical couplings of the primary and secondary bundles to the casing of the mixer are made to be strong and very precise.

Two different types of references are used to monitor the intensity of the light pulses coming out of each mixer, namely photomultipliers equipped with Harshaw NaI-Am pulsers and Hamamatsu S2662 photodiodes. Because of the temperature dependence of the NaI(TI) scintillation efficiency, the photomultipliers are housed in temperature controlied cabinets located outside the L3 magnet, about $30 \mathrm{~m}$ away from the BGO barrel, whereas the photodiodes are mounted on the BGO barrel structure itself and need not be decoupled from the mixers during transportation between the calibrati. )n site and the L.3 experimental hall. For each photodiode, the $59.5-\mathrm{keV} \gamma$ ray line of an ${ }^{241} \mathrm{Am}$ source allows us to monitor the gain of the amplifying chain.

The stability of the optical fiber system is estimated as follows: in each sector of 240 crystals connected to the same mixer, the xenon light amplitude measured in a given channel is normalized to the sum of the amplitudes in the sector. Over a period of 45 days, the mean variation of these relative amplitudes for one complete half-barrel is $0.2 \% \mathrm{rms}$. This demonstrates the quality of the optical fiber system and of the electronics.

\subsubsection{Energy calibration}

The calorimeter was calibrated at CERN in the SPS $\mathrm{X} 3$ beam, where an accuracy better than $1 \%$ was obtained. In turn, each of the two fully equipped half-barrels was installed on a rotating table. Sufficient statistical accuracy was achieved by recording about 1500 electrons for each crystal at 2, 10 and $50 \mathrm{GeV} / c$ momenta. Owing to the high resolution of the calorimeter, this measurement requires a well tested procedure and control of systematic deviations from the nominal energy deposition.

The firs. requirement is a beam spectrometer of adequate resolution, stability and momentum reproducibility comparable to the energy resolution of the BGO. We measured the magnetic field of the bending 
Table 11

Parameters of the $\mathrm{X} 3$ beam spectrometer

\begin{tabular}{ll}
\hline Field reproducibility & $<0.15 \%$ \\
Field peak to peak ripple & $<10^{-4}$ at $10 \mathrm{GeV} / c$ \\
Field slow drift & $<5 \times 10^{-4}$ at $2 \mathrm{GeV} / c$ \\
$\begin{array}{l}\text { Chamber resolution } \\
\text { Absolute momentum error } \\
\text { from bending angle }\end{array}$ & $\pm 400 \mu \mathrm{m}$ \\
\hline
\end{tabular}

magnets to insure that the design parameters were respected. The measured parameters of the spectrometer are given in table 11.

The second requirement is to have a geometrical precision in the crystal alignment with the nominal beam direction and position such that no correction should be introduced in the evaluation of the energy deposited in the crystal. This requirement is translated in a positioning of the geometrical center of the crystal front face better than $1 \mathrm{~mm}$ and an angle between the beam and the crystal longitudinal axis less than $5 \mathrm{mrad}$. These limits were achieved by the mechanical precision of the supporting table and by the electronic controls of the servo motors.

Finally during the calibration the half-barrel was enclosed in an air-conditioned tent with a stabilized nominal temperature of $18 \pm 0.5^{\circ} \mathrm{C}$.

The calibration constant of a crystal is defined as the ratio between the energy deposited in the crystal and the electronic signal read by the readout system. The energy deposited $i$. a single crystal is a fraction of the electron energy which depends upon the impact point. In order to reduce this dependence, we sum the signals from a matrix of $3 \times 3$ crystals. In this sum we normalize each amplitude with the value of the signal given by the cosmic ray measurement. In subsequent iterations, the normalization values are replaced by the calibration constants obtained in the previous step.

Two quantities computed from the pulse height distributions can be used to evaluate the calibration constants: the peak and the mean values. We use the mean value to compute the constants and the peak to control systematic errors possibly due to data selection or to the influence of malfunctioning or absent channels. Both methods agree withiil $0.2 \%$ (fig. 95). Each crystal contributing to the tail of the distribution is reevaluated. A third method, based on single crystal signals, was also used as an aditional check. It agrees weil with the other methods $(<0.4 \%)$.

The main correction was due to the actual temperature profile in the BGO crystals. The thermal sensors allow the determination of a temperature map which is used to correre the measured pulse height applying the average temperature coefficient of $-1.55 \% /{ }^{\circ} \mathrm{C}$. The reproducibility anc' the stability of calibration constants

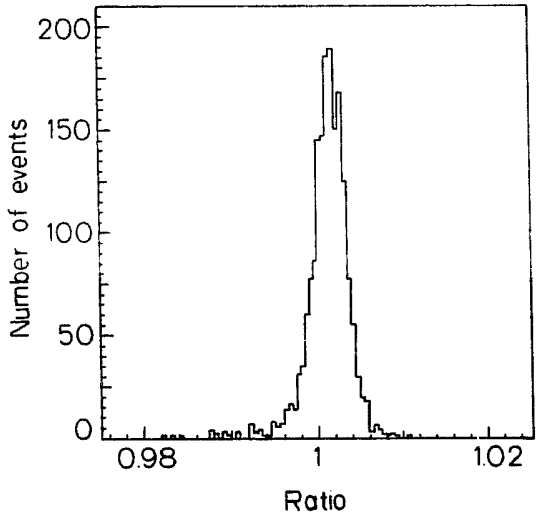

Fig. 95. Ratio of the mean value to the peak value for al crystals in the first half-barrel.

were tested with a prototype matrix [37], within $0.3 \%$ rms systematic error.

Since one of the most important parameters of the BGO detector is its low energy resolution, this was tested using one of the half-barrels at a specially designed beam line providing $180-\mathrm{MeV}$ electrons at the LEP injector linac. A representative sample of approximately 200 crystals were exposed to the beam. The measured resolution is shown in fig. 96 , which also include the high energy measurements as well as results from an early prototype detector. The resolution at $180 \mathrm{MeV}$ is better than 4\%, which is within the design goals of the detector.

Cosmic muons are used to monitor the calibration constants as measured at the test beam, and to perform periodic calibrations in situ to ensure the stability of the energy response of the calorimeter $[38,39]$. They allow a measurement of the possible variation of the light response of the crystal along its major axis. The expected muon rate useful to the calibration, i.e. those muons crossing opposite faces of a crystal, is about 500/day

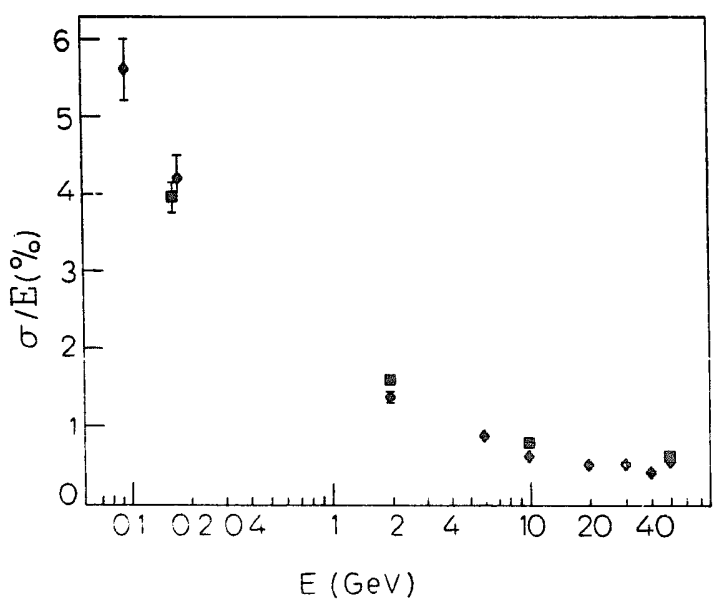

Fig. 96. Energy resolution. Diamonds: prototype 1985; squares: barrel 1988. 


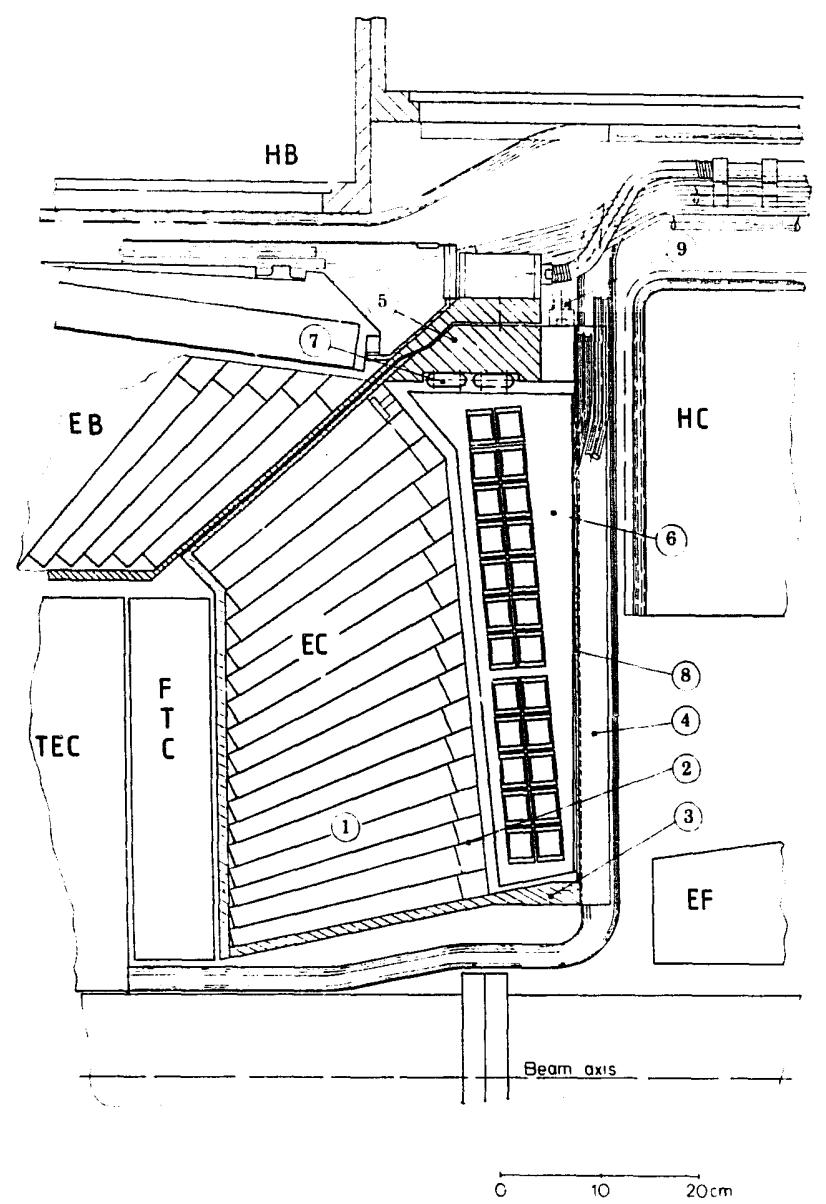

Fig. 97. BGO end cap longitudinal section. (1) Crystals. (2) Cell walls. (3) Mechanical structure. (4) Stiffening radial spokes. (5) Supporting ring. (6) Preamplifier board. (7) Cooling ducts.

(8) Thermal shield. (9) Optical fiber bundle.

per horizontal crystal and 200 /day per vertical crystal. With these rates, the precision on the muon momentum measurement in the muon chambers and the determination of the energy loss in BGO [40], calibration and monitoring of the light yield along the crystal with the accuracy required to match the detector performances can be achieved in a few days.

\subsection{The end caps}

The end caps (EC) are made of two symmetrical parts, with $1536 \mathrm{BGO}$ crystals each, giving an angular coverage ranging from $12^{\circ}$ to $42^{\circ}$ and $138^{\circ}$ to $168^{\circ}$, respectively (fig. 97). Each end cap is split into two halves for installation around the beam tube. Each half is composed of eight sectors (fig. 98), in which the crystals are distributed into five modules of three rows and one module of two rows near the beam tube. The end caps are built with the same materials as the barrel. The electronic readout, the thermal regulation system and the xenon light monitoring system are also identical

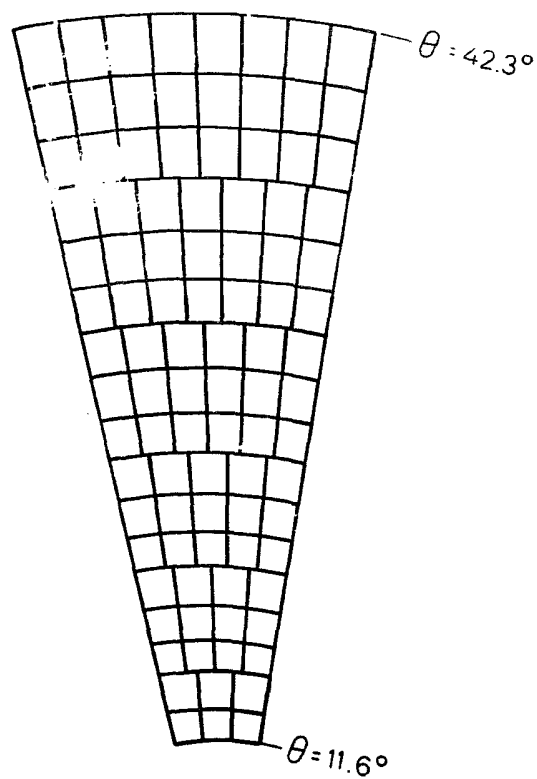

Fig. 98. Crystal arrangement in an end cap sector. There are 16 sectors per end cap.

to those used in the barrel. In front of each endcap, four drift chambers measure the position and the direction of a charged particle after the TEC flange with a spatial resolution of better than $200 \mu \mathrm{m}$, and angular precision better than $10 \mathrm{mrad}$.

\section{The luminosity monitor}

\subsection{Design description}

The luminosity monitor (fig. 99) is designed for reliable lumincsity measurements in the $Z^{0}$ energy range at LEP [41] by measuring the rate of Bhabha events. It

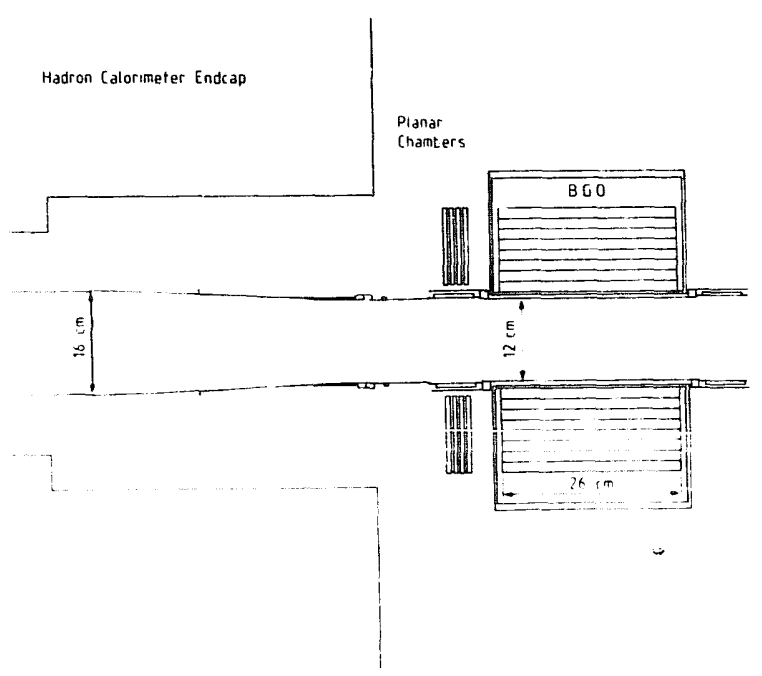

Fig. 99. The L3 lumirosity monitor. 


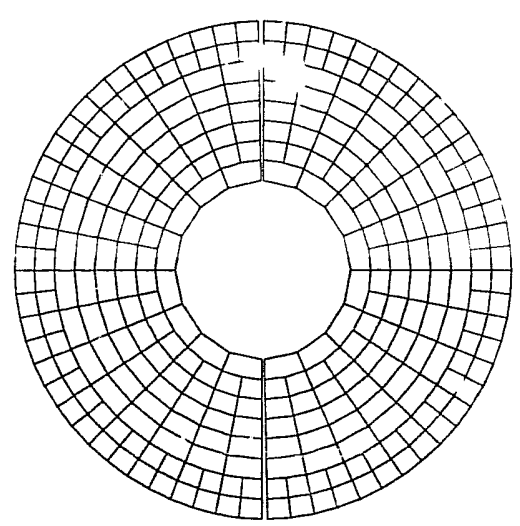

Fig. 100. An end view of the BGO crystal array.

is located in an angular region forward enough to become independent of the $\mathrm{Z}^{0}$ exchange and yet not too far forward so as to allow easy Bhabha event selection unaffected by systematic errors. It consists of a charged particle tracking device with good position resolution, followed by a highly segmented BGO array of good radiation hardness to measure the energy of the showering electrons and photons.

With this system, ore can study in detail the shabha process including the radiative tail. The trigger [42] will permit the measurement and rer.oval of ba-kground events like beam-gas interactions. Offline analysis will remove the Bhabha events that develop only a fraction of their energy in the BGO detector but otherwise pass the trigger condition. By comparing the tracking information with the energy profile deposited in the crystal array, one can define a very precise geometrical acceptance region. An asymmetric software cut will reduce the systematic effects of variations in the LEP beam parameters.

The BGO array is cylindrically symmetric. The crystals are arranged in eight rings (fig. 100), each covering $15 \mathrm{~mm}$ radially, parallel to the beam pipe. Azimuthally, they are arranged in 16 sectors of $22.5^{\circ}$ each. Each sector consists of 19 crystals which are 26 $\mathrm{cm}$ long and which range in cross section from $1.5 \times 1.5$ $\mathrm{cm}^{2}$ to $1.5 \times 3.0 \mathrm{~cm}^{2}$. The crystals are wrapped first in teflon tape and then in $25 \mu \mathrm{m}$ copper foil. Each crystal is viewed by an Hamamatsu photodiode, and a yellow light emitting diode is mounted opposite the photodiode to monitor any radiation camage and recovery. To ensure optimum shower containment, a software cut is defined that limits the acceptance to the inner six of the eight rings. This also matches the full efficiency range of the monitor chambers. The BGO array is split into two halves that are separated (fig. 101) during each filling of the LEP ring by an hydraulic device with a positioning accuracy of $10 \mu \mathrm{m}$. A lead shield between BGU and beam pipe provides further radiation protectior. Ti.e

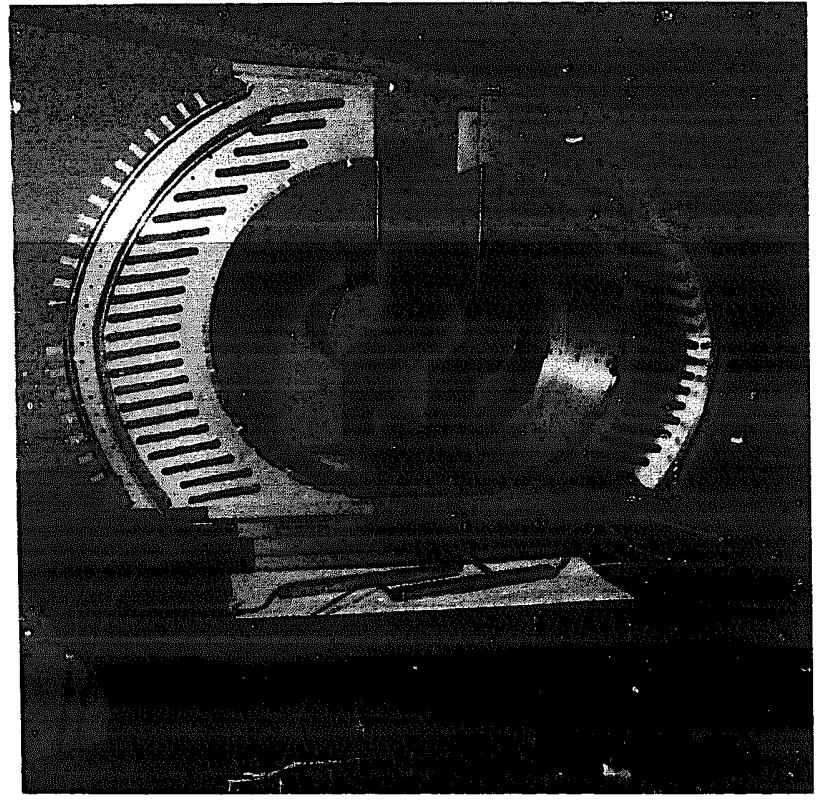

Fig. 101. The support structure for the BGO array.

main characteristics of the system are summarized in table 12.

Apart from an energy trigger demanding a large amount of energy deposited in the two BGO arrays (e.g. for tags in two-photon physics), a geometrical trigger will require a coincidence having a minimum energy in each of the two BGO arrays. The azimuthal width of the overlap region for the coincidence is defined as two BGO sectors, i.e. $45^{\circ}$. This trigger scheme ailows the

Table 12

Main characteristics of the L3 luminosity monitor

\begin{tabular}{|c|c|}
\hline Distance from the interaction point [cm] & 265 \\
\hline Beal, 1 pipe radius [cm] & 6.0 \\
\hline \multicolumn{2}{|l|}{ Radial extent of physical BGO array } \\
\hline $\begin{array}{l}\quad R_{\min }-R_{\max }[\mathrm{cm}] \\
\text { Radial extent of acceptance area }\end{array}$ & $6.8-19.0$ \\
\hline $\begin{array}{l}\quad R_{\min }-R_{\max }[\mathrm{cm}] \\
\text { Effective polar angle coverage }\end{array}$ & $8.8-17.5$ \\
\hline$\Theta_{\min }-\Theta_{\max }[\mathrm{mrad}]$ & $31-62$ \\
\hline Effective Bhabha cross section $\sigma$ [nb] & 100 \\
\hline Length of BGO crystal [cm] & 26.0 \\
\hline Length of BGO crystal $\left[X_{0}\right]$ & 24 \\
\hline \multicolumn{2}{|l|}{$\begin{array}{l}\text { Tracking chamber resolutions } \\
\text { (entire track) }\end{array}$} \\
\hline$\Delta R$ a) $[\mu \mathrm{m}]$ & $<250$ \\
\hline$\Delta \Theta[\mathrm{mrad}]$ & $<0.10$ \\
\hline$\Delta \Phi^{\text {a) }}[$ deg] & 0.6 \\
\hline \multicolumn{2}{|l|}{ Calorimetry } \\
\hline$\Delta E / E^{\text {a) }}[q]$ & $0.5-1.0$ \\
\hline$\Sigma P^{\text {a) }}[\mu \mathrm{m}]$ & $<800$ \\
\hline$\Delta \Theta[$ mrad $]$ & $<0.3$ \\
\hline$\Delta \Phi^{\text {a) }}[\mathrm{deg}]$ & $<n$ \\
\hline
\end{tabular}


observation of radiative and nonradiative Bhabha events as well as of background interactions. Software studies will then control the amount of background to be admitted into the luminosity event sample.

The forward tracking system in front of the BGO array consists of a stack of four planar multiwire proportional chambers with cathode strip readout. The chamber dimensions are $400 \mathrm{~mm} \times 200 \mathrm{~mm} \times 10 \mathrm{~mm}$ and each stack of four chambers has the cathode strips arranged in both $R-\Phi$ (two chambers) and $x-y$ (two chambers) configurations. Data on efficiency and spatial resolution were obtained by exposing a stack of four prototype chambers to $50 \mathrm{GeV}$ electrons in the $\mathrm{X} 3$ beam at the CERN SPS. The efficiency per wire plane was better than $98 \%$. Requiring a track to have at least 3 out of 4 hits gives a tracking efficiency of $99.8 \%$. The spatial resolution was $<350 \mu \mathrm{m}$ per chamber. Again, requiring at least 3 out of 4 hits gives a spatial resolution per track of better than $250 \mu \mathrm{m}$. The measured pulse height matching between opposed cathode planes within an individual chamber has an rms spread of $17 \%$; this will aid in resolving ambiguities for multihit events.

Because of the steep dependence of the Bhabha cross section on the angle from the beam axis a crucial factor is the turn-on of the efficiency near the inner radius of the chambers. 'The system was found to be fully efficient for radii greater than $88 \mathrm{~mm}$ which is adequate for the fiducial voiume used in the energy trigger.

\subsection{Precision goals for the L3 luminosity monitor}

The luminosity monitor will accept an angular reginn of 30-62 mrad with full efficiency, corresponding to an effective Bhabha cross section $\sigma \approx 100 \mathrm{nb}$. At an average luminosity of $10^{31} \mathrm{~cm}^{-2} \mathrm{~s}^{-1}$, a trigger rate of about $1 \mathrm{~Hz}$ will result, to be compared with the $0.3 \mathrm{~Hz}$ expected rate from $\mathrm{Z}$ events. Thus, a statistical error of about $1 \%$ in the luminosity will be achieved in a $3-\mathrm{h}$ run. We aim at a precision, including systematic effects, of better thar $2 \%$ in the integrated luminosity on a run to run basis. The main limitation will then come from systematic errors which can be separated into four major sections, as discussed in the following subsections.

\subsubsection{Theoretical uncertainties}

These include uncertainties in the Bnabha cross section, such as weak interaction effects, vacuum polari ation, or multiple photon emission beyond ouder $a^{3}$. They contribute to the systematic errors for an estimated $1 \%$.

\subsubsection{Detector performance}

The limitations of the luminosity detector contribute below the $1 \%$ level to the systematics. The total energy trigger and the requirement of good lateral shower containment will produce no significant loss of Bhabha events. Longitudinal shower containment is almost complete in 24 radiation lengths and can be ignored against the more incomplete lateral containment. The tracking chamber resolution of better than $250 \mu \mathrm{m}$, taken at the critical inner radius $R=8.8 \mathrm{~cm}$, produces a luminosity error of less than $0.6 \%$ per event and this will be made negligible by the statistics of a 3-h rur. Chamber production tolerances, as well as final alignment and survey, should be below $100 \mu \mathrm{m}$ and thus contribute $0.2 \%$ to the systematics. The chambers will be mounted in a tixed position on the beam pipe and they will be surveyed with respect to the LEP quadrupoles. Chamber efficiencies have the most critical effect in the systematics. They must be known to better than $1 \%$ per wire, and must also be stable at that level. Requiring hits on 3 out of $\mathbf{4}$ chambers reduces the systematic error to $0.3 \%$. Thus, by monitoring any inefficiencies, especially near the onset of full efficiency at $R=8.8 \mathrm{~cm}$, the systematic error shculd be well below the $1 \%$ level.

\subsubsection{LEP bearn backgrounds}

Minor contributions to the systematics come from background interactions mixed into the event sample. Synchrotron radiation induced events are easily removed by a suitable energy threshold in the trigger. Off-momentum electrons resulting from beam-gas or beam-wall interactions will be studied as part of the event sample. They are identifiable as highly acoplanar events wh: h violate the geometric trigger but are accept d by the energy trigger. A study of these events will permit a ieliable backgrouna subtraction accurate to better than the $1 \%$ level.

\subsubsection{LEP beam parameters}

One of the most serious obstacles to keeping the luminosity systematics below the $1 \%$ level is the dependence of the observed Bhabha rates on variations of the beam parameters at the interaction point. The parameters are predictions derived from single separated beam measurements. Hence, a major effort was undertaken to adequately eliminate the dependence of the Bhabha calibration on the precise values of the beam parameters. After consultation with the LEP instrumentation group, the following parameters vere picked as the most essential to control [43]: IP position $\langle\langle x\rangle,\langle y\rangle$, $\langle z\rangle)$; IP width $\left(\left\langle\sigma_{x}\right\rangle,\left\langle\sigma_{y}\right\rangle,\left\langle\sigma_{z}\right\rangle\right)$; beam dispersion $\left(\left\langle\sigma_{x^{\prime}}\right\rangle,\left\langle\sigma_{y^{\prime}}\right\rangle\right)$; and angular beam offset $\left(\left\langle x^{\prime}\right\rangle,\left\langle y^{\prime}\right\rangle\right)$.

A Monte Carlo study of the effects of beam parame. ter values, in comoination with the trigger design and event selection, was undertaken [42]. We have foresezn the use of an asymmetric softwart trigger [44]: one of the detector arms requires the nominal geometrical trigger between 30 and $62 \mathrm{mrad}$, whereas the other arm has to satisfy the geometrical trigger within a loosened angular range from $30-d$ to $62+d$ milliradians, where 


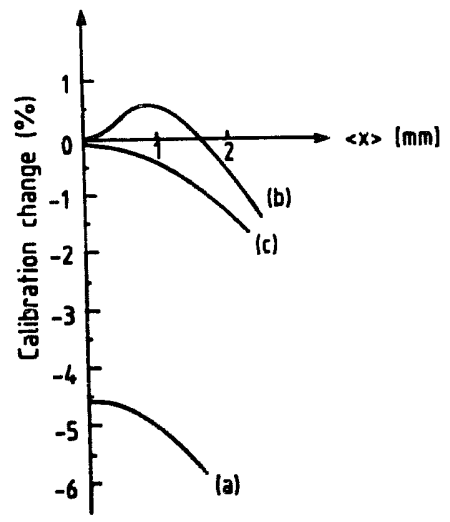

a) $d=0, \sigma_{x}, \sigma_{y}, \sigma_{z}$ as for (c):

b) $d=0.6 \mathrm{mrad}, \sigma_{x}=\sigma_{y}=\sigma_{z}=0$

c) $d=0.6 \mathrm{mrad}, \sigma_{x}=0.3 \mathrm{~mm}, \sigma_{y}=0.012 \mathrm{~mm}, \sigma_{z}=33 \mathrm{~mm}$ -

Fig. 102. Effect of asymmetric trigger.

$d$ is a parameter to be suitably chosen. As an example, the event rates drop almost linearly with $\langle x\rangle$ when $d=0$. With increasing $d$, the dependence becomes parabolic until, for a particular value of $d$, there is no dependence for a wide range of $\langle x\rangle$ values With still larger $d$ values, one obtains even a rise in rate with $\langle x\rangle$ for small values of $\langle x\rangle$. The variation of the event rate for particular values of $d$ as a function of $\langle x\rangle$ is shown in fig. 102 .

In our sirulation, a chanber resolution of $400 \mu \mathrm{m}$ was used. The energy showers were not simulated, but lateral non-fiuctuating shower spreading was allowed. The trigger was simulated with an energy trigger threshold of $78 \%$ of beam energy per arm. The events ihemselves were produced by a standard generator [45] including first order radiative events.

It is important to realize that the resuits from the study on the systematic errors apply only in case that a particular beam parameter setting cannot be measured (or predicted). If a parameter can be measured (e.g. by

Table 13

Systematic uncertainties in relative luminosity measurement

\begin{tabular}{llll}
\hline $\begin{array}{l}\text { Parameter } \\
\text { at IP }\end{array}$ & $\begin{array}{l}\text { Typical } \\
\text { value }\end{array}$ & $\begin{array}{l}\text { Known } \\
\text { to }\end{array}$ & $\begin{array}{l}\text { Absolute } \\
\text { change in \%o } \\
\text { for typical value }\end{array}$ \\
\hline$\langle x\rangle$ & $100 \mu \mathrm{m}$ & $15 \mu \mathrm{m}$ & 0.1 \\
$\sigma_{z}$ & $300 \mu \mathrm{m}$ & $10 \mu \mathrm{m}$ & 1.5 \\
$\langle y\rangle$ & $100 \mu \mathrm{m}$ & $5 \mu \mathrm{m}$ & 0.1 \\
$\sigma_{y}$ & $12 \mu \mathrm{m}$ & $1 \mu \mathrm{m}$ & 0.06 \\
$\langle z\rangle$ & $1 \mathrm{~mm}$ & $0.7 \mathrm{~mm}$ & 0.1 \\
$\sigma_{z}$ & $33 \mathrm{~mm}$ & $0.5 \mathrm{~mm}$ & 1.5 \\
$\left\langle x^{\prime}\right\rangle$ & 0 & $2 \mu \mathrm{rad}$ & 0 \\
$\sigma_{z^{\prime}}$ & $175 \mu \mathrm{rad}$ & $5 \mu \mathrm{rad}$ & 0.05 \\
$\left\langle y^{\prime}\right\rangle$ & 0 & $10 \mu \mathrm{rad}$ & 0 \\
$\sigma_{y^{\prime}}$ & $175 \mu \mathrm{rad}$ & $5 \mu \mathrm{rad}$ & 0.05 \\
\hline
\end{tabular}

the central detec or), it can clearly be corrected for and its effects on systemnatics removed. A study is under way to investigate the feasibilicy of measuring beam parameters with the luminosity monitor itself. Hence, the stated results are worst case only. Table 13 shows typical values for the parameters as they have been estimated by the LEP instrumentation group [43]. The column labelled "absolute change" shows the corresponding change in the luminosity calibration. Using a value of $d=0.6 \mathrm{mrad}$, the systematic error on the absolute luminosity due to ignorance of beam parameter settings can be kept below the $1 \%$ level for values several times larger than the column labelled "typical value" in table 13.

\section{The central track detector}

\subsection{Introduction}

The L3 central track detector is designed with the following goals:

- detection of charged particles and precise measurement of the location and direction of their tracks;

- determination of the transverse momentum and the sign of the charge for particles up to $50 \mathrm{GeV} / \mathrm{c}$;

- reconstruction of the impact point and direction for charged particles at the entrance of the electromagnetic calorimeter;

- determination of the track multiplicity originating from the interaction region at the trigger level;

- reconstruction of the interaction point and of secondary vertices for particles with lifetimes greater than $10^{-13} \mathrm{~s}$.

These goals and the limited space available for this detector within the electromagnetic calorimeter have determined its design. The total lever arm available for coordinate measurements in the chamber is $37 \mathrm{~cm}$ radially. The charge identification of $50 \mathrm{GeV} / c$ particles with $95 \%$ confidence requires 50 coordinate measurements with $50 \mu \mathrm{m}$ resolution. This is accomplished by two concentric cylindrical drif: chambers on common end plates sperated in "time expansion" mode, the TEC, surrounded by two cylindrical proportional chambers with cathode strip readout, the Z-detector (fig. 103).

\subsection{The time expunsion chambar}

Following the TEC principle, the high field amplification region at the sense wire piane is separated from the low field drift region by an additional grid wire plane. This configuration allows to optimize the electron arrival time distribution as well as the track length seen by the individual anode wires independently of the drift velocity chosen in the drift region. The TEC oper- 


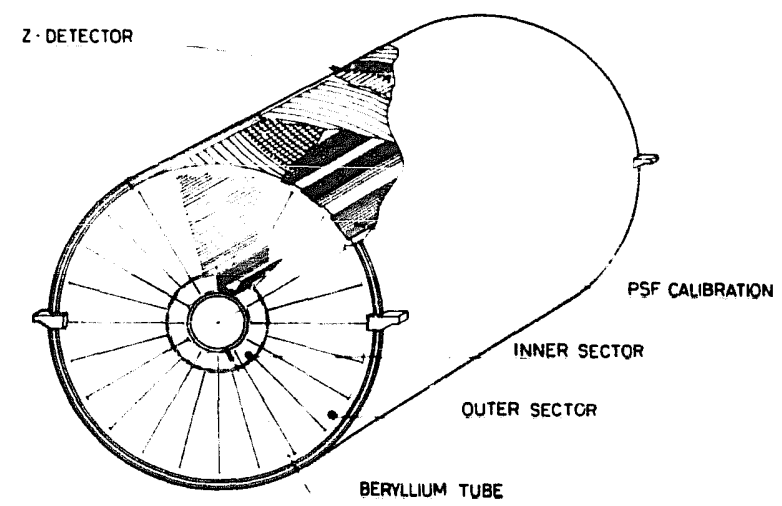

Fig. 103. General view of the central track detectors.

ates with $80 \% \mathrm{CO}_{2}$ and $20 \% \mathrm{iC}_{4} \mathrm{H}_{10}$, which has a low longitudinal diffusion and thus permits a low drift speed. Furthermore, this gas has a negligible Lorentz angle. To reach the ultimate resolution for drift lengths of up to $5 \mathrm{~cm}$, determination of the drift time by a center of gravity method is mandatory. Thus the anode pulses are sampled by Flash Analog to Digital Converters (FADC) after a shaping of the analog pulses to cancel the ion tail. This principle has been tested by prototype chambers in test beams and in the MARK $J$ experiment at PETRA [46-48].

\subsubsection{Mechanical construction}

The TEC wires are supported by two end plates with precisely drilled holes for the anode, potential and field shaping wires. Furthermore, the plates contain the gas inlets and outlets, and the grooves for the positioning of the grid wire planes (fig. 104). The end plates are held apart, and the enclosure is gas sealed, by a 4-mm thick aluminum cylinder at the outer diameter and a $1.5-\mathrm{mm}$ thick beryllium cylinder at the inner diameter. The structure has been studied by the finite element technique to meet the required precision of about $10 \mu \mathrm{m}$ for the wire positioning and to minimize the amount of material traversed by the particles entering the chamber and reaching the electromagnetic calorimeter (less than $1 \%$ and $10 \%$ of a radiation length, respectively). Fig. 105 shows one of the end plates.

The anode wire planes are arranged radially forming 12 inner and 24 outer sectors. Their sensitive length is $982 \mathrm{~mm}$. There are two types of sense wires: standard wires to measure precisely the $R-\Phi$ coordinates of the tracks and charge division (CD) wires to determine the $Z$ coordinates. Additionally, for part of the standard wires, groups of five grid wires on each side of the amplification region are read out (pick-up wircs). Comparing the induced signals, the left-right ambiguity for these anodes (LR wires) can be resolved [49]. The inner sectors include six standard and tw, $C D$ wires, while the outer sectors include 31 standard, 14 LR and 9 CD wires.

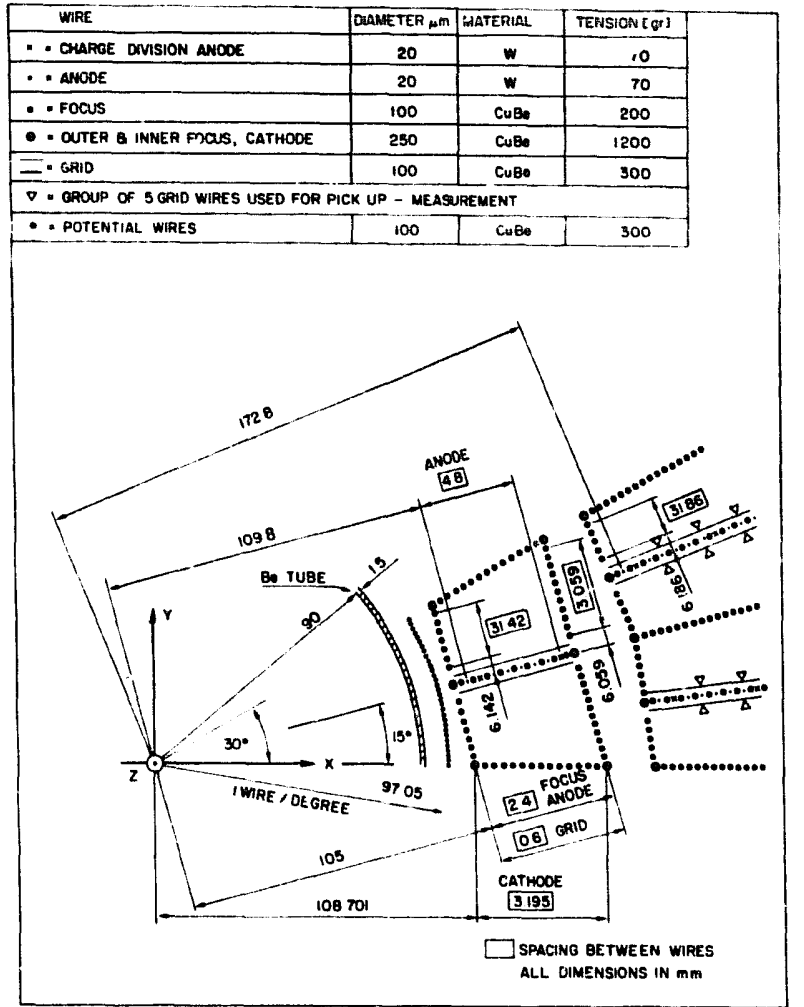

Fig. 104. Technical drawing of the wire configuration in one inner TEC sector and in part of two cuter sectors.

\subsubsection{Readout}

Fig. 106 shows a schematic drawing of the analog part of the TEC readout. All anodes arr capacitively coupled to the same type of hybrid preamplifiers via $\mathrm{HV}$ protection circuitry. The $\mathrm{CD}$ anodes have an increased time constant for better signal-to-noise ratio. The shapers (type A) are set to produce a symmetric

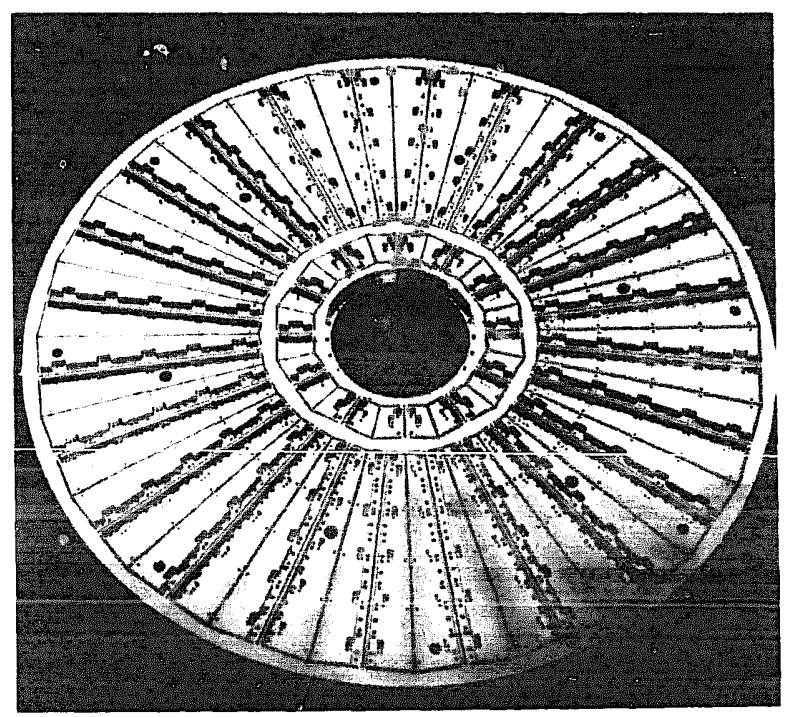

Fig. 105. One of the TEC end plates. 


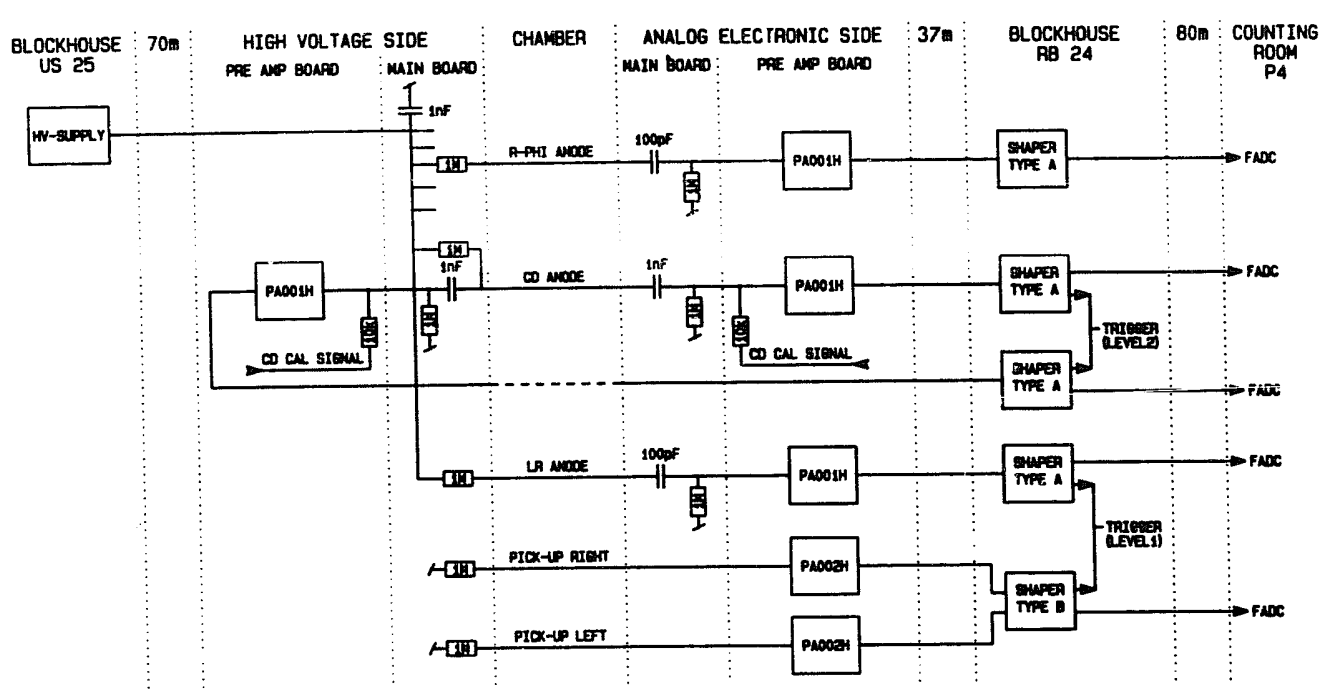

Fig. 106. Schematic diagram of TEC analog electronics.

output signal with minimal overshoot [50]. For the pick-up (grid) wires, a different preamplifier and a special shaper (type B) with a differential input are used. The shapers for the LR anodes, the pick-up wires and the $C D$ anodes provide additional output signals to the level 1 and level 2 trigger processors. Calibration of the CD channels is done by injecting known charges at the corresponding preamplifier inputs.

The design parameters of the digital readout system of the TEC are given by the $45-\mathrm{kHz}$ bunch crossing rate and the $50-\mathrm{Hz}$ events rate accepted by the trigger level 2. To achieve this, the readout system has to buffer 10 events at trigger level 1 . The $2 \mathrm{Mb}$ of raw data per TEC event, coming from 2000 readout channels, have to be reduced considerably before being transferred to the $\mathrm{L} 3$ data acquisition system. Therefore, a fast data reduction processor (DRP) is attached to each pai- of digitization units.

The TEC readout system (fig. 107) consists of 60 VME crates organized in 4 chains of 15 crates. Each crate houses up to 38 FADC channels and its control unit, the crate master (CM). In a fifth chain, the FADC units are replaced by charge to time converters (QTC) for readout of the $Z$ chambers and by multiplexers to read the scintillating fibers. Special modules have been designed to interface the crates with the monitoring computer, the $\mathrm{L} 3$ trigger system and the DAQ system. The TEC data are written into a LeCroy 1892 FASTBUS memory where they are picked up by the L3 DAQ system. A program running on the host computer initializes and supervises the complete system.

The shaper output signal of every anode wire is digitized by one FADC with a $100-\mathrm{MHz}$ clock rate. Each FADC consists of a 6-bit ADC chip (TRW TDC 1029-J7C) and 1-kB ECL memory (Hitachi HM2112-1). This allows one to digitize the incoming puises in 10.24 $\mu s$, covering the maximum TEC drift time. The FADC input can be switched under program control to a signal generated by the crate master for calibration. Two FADC channels are connected to one DRP to reduce the amount of data transferred downstream in the readout system. The two FADC channels and one DRP are built as one VME board (fig. 108).

The DRP is built around a Texas Instruments chip TMS 99105 with a $24-\mathrm{MHz}$ clock. It has a 32-kByte RAM memory for program, data and multievent buffering. Started by a trigger level 2 accept, the DRP reduces the FADC raw data to about 5\%. The output is written into a dual port memory from where it is collected by the crate master (CM). For a typical TEC event, the computation time is less than 5 ms. A check sum can be

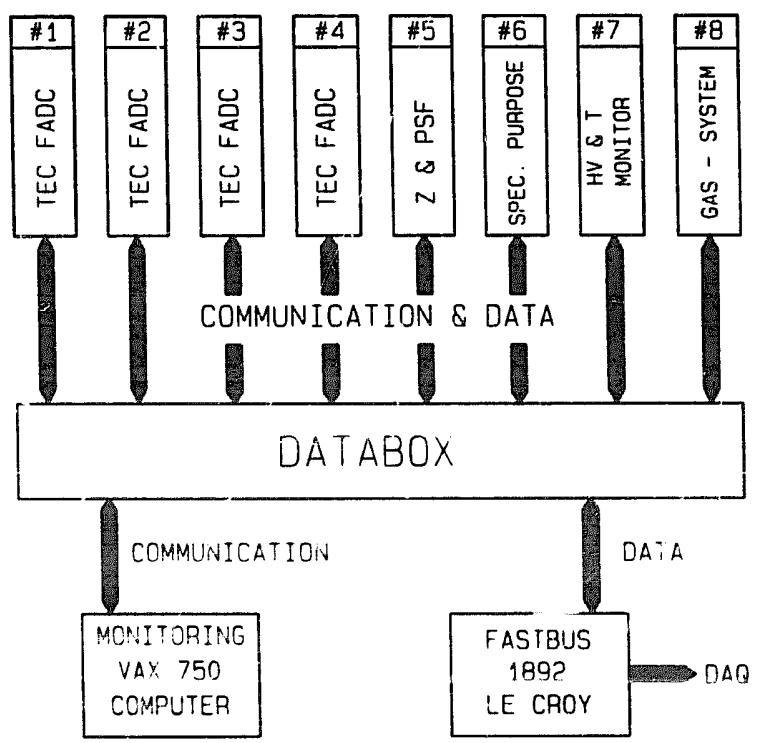

Fig. 107. Schematic diagram of the certral track detector readout systems. 


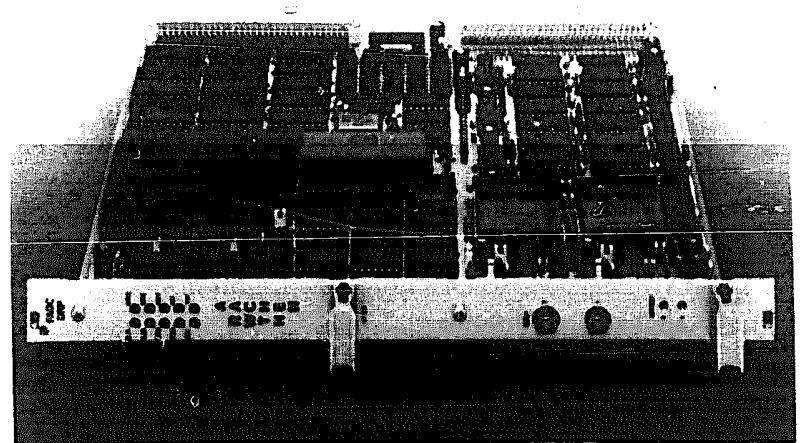

Fig. 108. A VME module containing two Flash ADC channels and one DRP.

calculated by the DRP and added to its output.

Each VME crate is controlled by a CM, which consists of three PC boards. It is built around a Motorola $6800012-\mathrm{MHz}$ CPU. The central function of the CM is to communicate with the DRP units in the crate and to transfer the DRP data to the data acquisition system. The CM also contains the FADC main control unit (FMC) which accepts the primary trigger input signals and controls all FADC functions. The data collected by the CM from all DRP are stored in a 64-kByte output memory (OM). The $O M$ of 15 crates build a chain which is connected to the main DAQ system through a fast readout sequencer and the FASTBUS memory module.

A special VME crate, the databox, contains modules which control the flow of data from the CM to their destinations. The readout scanner module (RS) feeds the data from the $\mathrm{OM}$ to the receiving memory module. A lookup table flags crates that have to be skipped in the readout and crates whose output data should be copied into a spy channel. Three spy channels equipped with 256-kByte memory each enable the monitoring program to investigate TEC data without interfering with the main data stream.

\subsubsection{Infrastructure}

To achieve the spatial resolution of $50 \mu \mathrm{m}$, the TEC is opurated with a gas mixture of $80 \% \mathrm{CO}_{2}$ and $20 \%$ $\mathrm{iC}_{4} \mathrm{H}_{10}$, a gas with a low drift velocity, at 2 bar. The drift velocity, and hence the spatial resolution, depends strongly on the pressure and the stability of the gas mixture, thus the $\mathrm{iC}_{4} \mathrm{H}_{10}$ content must be stable within $0.1 \%$. Fig. 109 summarizes the design of the TEC gas system, which operates in closed loop to guarantee long term stability. The mixture is stored in a $1 \cdot \mathrm{m}^{3}$ barrel and circulated through a filter and an Oxysorb purifier to keep the oxygen content below $1 \mathrm{ppm}$. The purified gas passes through TEC at a flow of $200 \mathrm{l} \mathrm{bar} / \mathrm{h}$. The gas quality is continuously monitored by a mass spectrometer and the water content is measured by an hy:rometer. A small drift chamber monitors changes in drift velocity, efficiency and mean pulse height due to small changes in the gas composition. A VME computer supervises the gas system and passes status information to the monitoring computer and to the L3 slow control system.

The high voltage system supplies the resistor chains for the field shaping wires, as well as the anode and potential wires. Since the TEC is operated with a low drift velocity, linearly dependent on the value of the electric field, a. stability better than $0.5 \mathrm{~V} / \mathrm{cm}$ is necessary. For a safe start, the 210 power supplies are ramped at a speed of $100 \mathrm{~V} / \mathrm{s}$. Since the high voltage resistor chains and the preamplifiers are attached to the end plates of the chamber, their temperature has to be controlled by a cooling and heating system. There are 96 temperature sensors distributed over the inner and outer cylinders, as well as over the end plates. The three magnetic field components are also measured at two positions on each end plate.

\subsubsection{TEC calibration}

Each TEC segment is equipped on its outer surface with a plastic scintillating fiber ribbon to monitor the low drift velocity $(6 \mu \mathrm{m} / \mathrm{ns})$ to an accuracy of $0.1 \%$ (fig. 110). A ribbon is comprised of 143 fibers, each $700 \mu \mathrm{m}$ wide, $1000 \mu \mathrm{m}$ thick and $1.3 \mathrm{~m}$ in length. The fibers in each ribbon are read out using two multianode microchannel photomultiplier tubes. The signals from each tube are multiplexed serially using the MX4 microplex chip and are interfaced to the standard data reduction processor of the TEC [51].

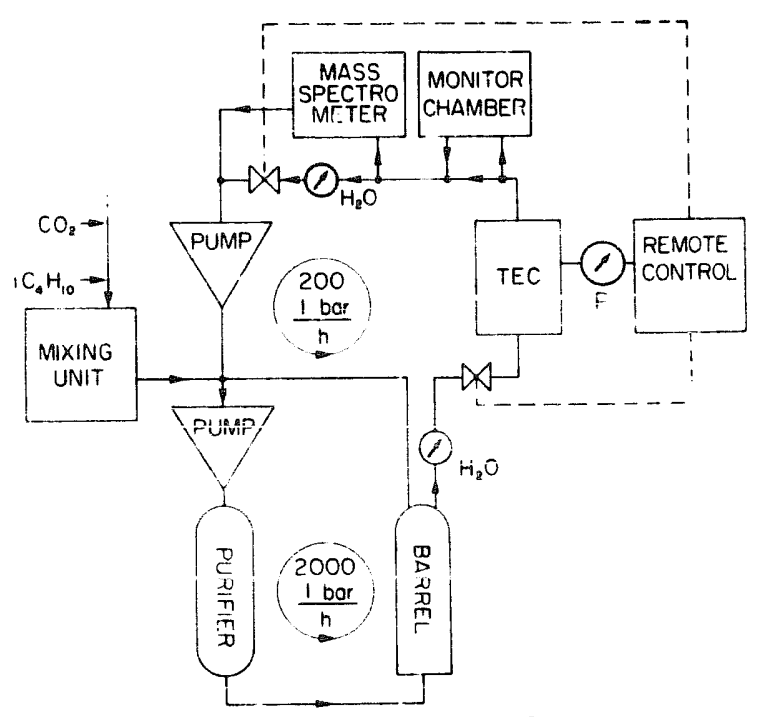

Fig. 109. Schematic diagram of TEC gas system. 


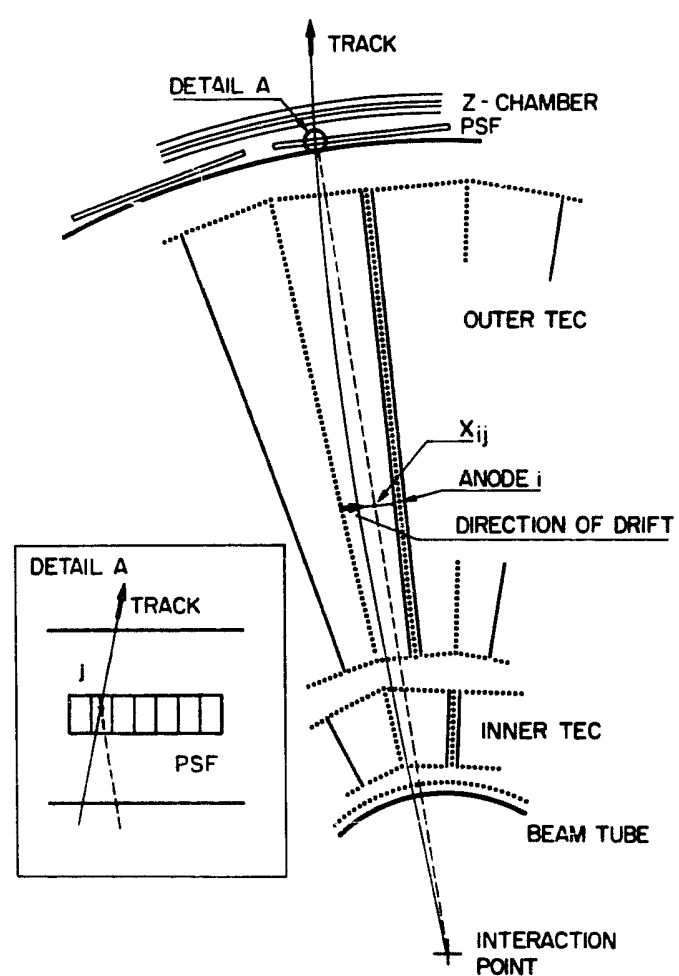

Fig. 110. TEC calibration using plastic scintillating fibers (PSF).

The drift distance-time relationship is obtained for every anode by averaging over the fitted tracks for every fiber using the $\mathrm{e}^{+} \mathrm{e}^{-}$interaction point and the fiber position. For every anode, an average drif: time is plotted for all fibers of the segment. Since each fiber yields azimuthal position information (with an rms of $700 \mu \mathrm{m} / \sqrt{12}$ ), the slope of such a plot is the inverse of the drift velocity of the specific anode. Monte Carlo studies have shown that for five tracks per fiber (6-h run at a luminosity of $10^{31} \mathrm{~cm}^{-2} \mathrm{~s}^{-1}$ ) the drift distance resolution is of the order of $10 \mu \mathrm{m}$, determining the drift velocity for each anode with a $0.1 \%$ accuracy.

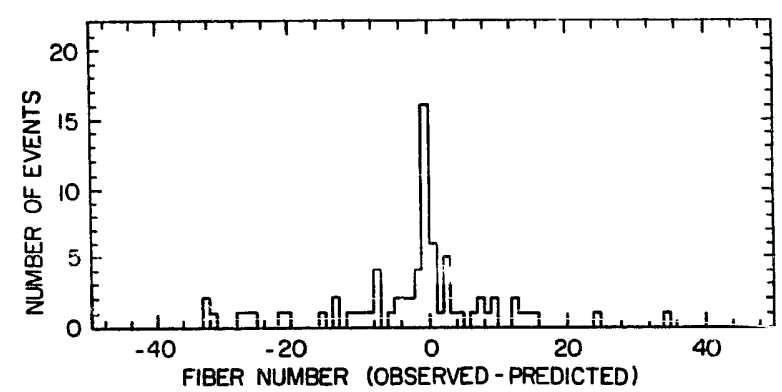

Fig. 111. Difference between the fiber position with a hit from the predicted fiber position using drift time information.

The feasibility of this calibration system was demonstrated in a test beam during March 1989 [52]. Fig. 111 shows the difference between the positions of fibers with a hit and the predicted fiber positions using the drift time information of the nearest anode wire of TEC. These residuals peak about one fiber position. The residuals at large deviations are due to the presence of more than one beam track per trigger.

\subsubsection{Test resuits from prototypes}

Since the most important design goal, an average resolution of $50 \mu \mathrm{m}$ in the measurement of the $R-\Phi$ coordinate, was reached on a prototype [47], much time has been spent to understand the variation of this resollution:

- The sampled track length influences the width of the electron arrival time distribution and the number of primary electrons collected at the anode. An acceptance of about $2.4 \mathrm{~mm}$ was found to be a good compromise.

- As the polar angle $\Theta$ deviates from $90^{\circ}$, the resolution improves due to increased electron statistics (fig. $112 \mathrm{a})$. When the azimuthal angle $\Phi$ deviates $\mathrm{fr}, \mathrm{m} 0^{\circ}$ with respect to an anode plane, the resolution deteriorates due to a stretching of the arrival times. This effect is small for the L3 TEC, since the maximum value of $\Phi$ is $7.5^{\circ}$ (fig. 112b).
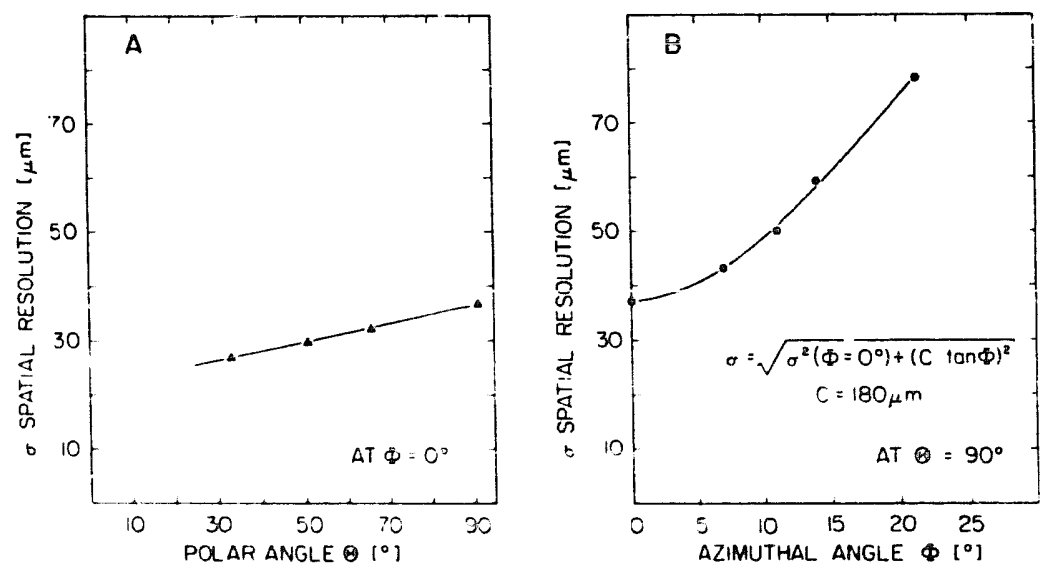

Fig. 112. Impact of changes in the polar angle (A) and in the azimuthal angle (B) on the $R-\Phi$ resolution. 
- The range of drift velocities is given on the one hand by the wire geometry and the resulting high voltages and on the other by the possible 8-bunch operation of LEP. Inside this range, a measurement of the resolution as a function of drift velocity indicated an optimum of about $6 \mu \mathrm{m} / \mathrm{ns}$.

- A tuning of the shaping amplifier for symmetric output and the use of a $80 \% \mathrm{CO}_{2}-20 \% \mathrm{iC}_{4} \mathrm{H}_{10}$ gas mixture at 2 bar gives an optimum between resolution and running conditions.

A Z-coordinate resolution of about $2 \mathrm{~cm}$ was reached by charge division. The same $R-\Phi$ resolution was obtained from the $C D$ wires as from the standard anodes. The left-right ambiguity is resolved by comparing signals induced on the neighboring grid wires [49]. These signals can also be processed and read out by the FADC/DRP system and be used in the trigger system.

Because of the restricted radial extension of the TEC, the two track resolution is important. It was studied by superimposing single hits from test beam events. Beyond a separation of $450 \mu \mathrm{m}$ in drift distance, fully efficient two track resolution can be expected [53].

\subsubsection{Results from test beam}

The complete detector system has been tested in a pion beam at CERN during March 1989. The components were operated under standard conditions as foreseen for LEP operation, including proper temperature and cable lengths.

The quality of the TEC signals was monitored by switching several DRP to "raw data mode" to display the full DRP memory content from the events. Fig. 113 demonstrates the excellent performance of the digitized signals after pulse shaping from a multitrack event. The

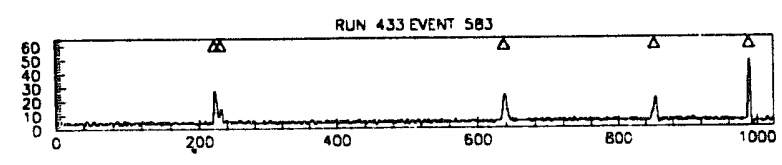

FADC + ROW ORP 1117 Sol: 15201044

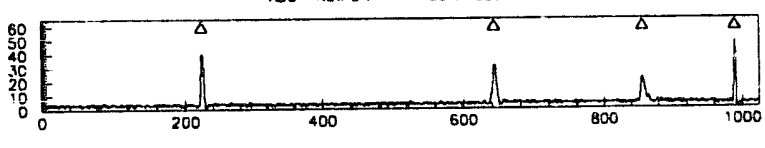

FADC 2 ROW DRP 1117 SOtt 15201045
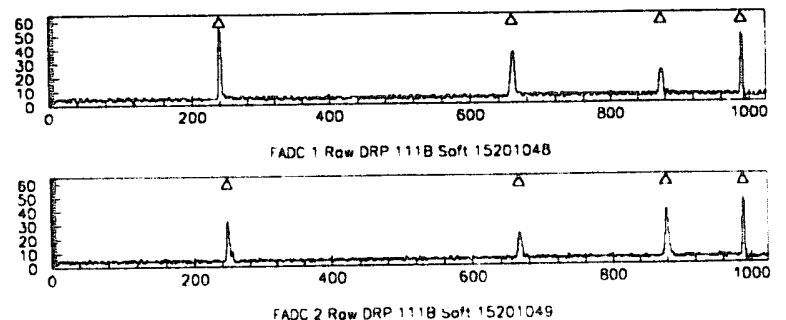

Fig. 113. Display of FADC raw data for a multitrack event and the time marker sign 1 (extreme right). The triangles indicate the peak position found by the peak-finding program.

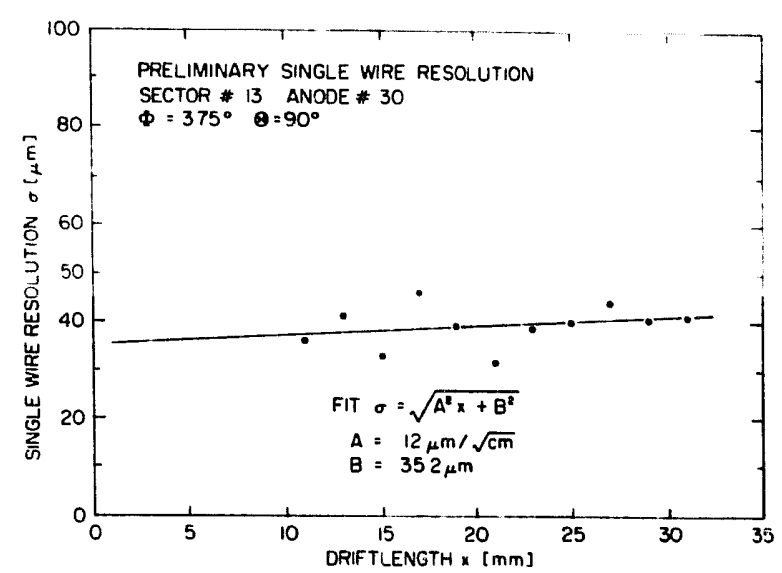

Fig. 114. Single wire resolution from the time expansion chamber operated at nominal conditions in a $15 \mathrm{GeV} / c$ pion test beam.

pulse at the extreme right is the time reference signal. The triangles indicate the peak positions found offline by the same peak finder program running in the DRP operating in normal mode. Fig. 114 shows an example of the single wire resolution as a function of the track distance from the anode. This has been obtained with a straight line fit on drift times determined from the DRP information in one of the TEC sectors. The average resolution stays well below the design goal of $50 \mu \mathrm{m}$.

\subsection{The $Z$ detector}

The $Z$ detector (fig. " 10 ) consists of two cylindrical proportional chambers with cathode strip readout. covering the outer cylinder of the TEC (fig. 103). The strips of the four cathodes form angles of $90^{\circ}, 70.1^{\circ}$. $-70.1^{\circ}$ and $0^{\circ}$ with respect to the beam direction, respectively. The three supporting cylinders are built of polyurethane foam as filling material, reinforced by

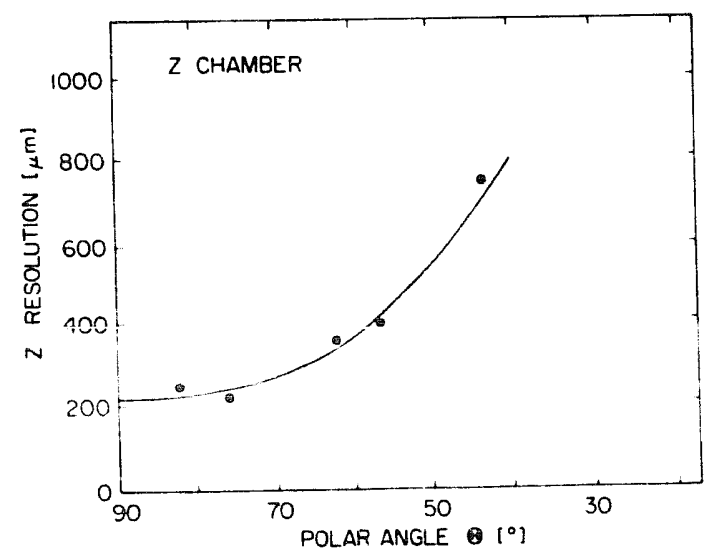

Fig. 115. Spatial resolution of a $Z$ detector prototype as a function of polar angle. 
$200-\mu \mathrm{m}$ glass fiber epoxy. Both cover layers of the middle cylinder and the outer (inner) layer of the inner (outer) cylinder consist of $50-\mu \mathrm{m}$ Kapton foil carrying the aluminum cathode strips of 4.4-mm pitch. On both ends of the cylinders, two glass fiber reinforced epoxy rings hold the wire feedthroughs. In order to avoid electrostatic displacements, the chamber is operated in drift mode with every second wire at ground potential. The $Z$ detector has an effective length of $1068 \mathrm{~mm}$, an outer diameter of $980 \mathrm{~mm}$ and a thickness of $21.5 \mathrm{~mm}$, representing about $1.3 \%$ of a radiation length. An $80 \%$ argon and $20 \% \mathrm{CO}_{2}$ gas mixture is used for operation [54].

The high voltage is supplied on one end of the cylinder; the signals from the 920 cathode strips are read out from the opposite end. Preamplifier boxes carrying four 4-fold hybrid circuits are positioned on the conical end flange. The signals are fed via $45 \mathrm{~m}$ coaxial cable, line drivers and another $70 \mathrm{~m}$ twisted pair cable to the ADC inputs. The digital readout forms part of the TEC readout system (fig. 107), the FADC/DRP units being replaced by 16 -fold 8-bit ADC. A calibration system will per iorm a quick check of all channels, measure the gain and rms noise of individual channels and the crosstalk between them.

The resolution as function of $\Theta$ was studied with a prototype of nearly the same length $(828 \mathrm{~mm})$ but with a reduced diameter $(246 \mathrm{~mm})$ (fig. 115). The average single track resolution over the full range $45^{\circ} \leq \Theta \leq$ $135^{\circ}$ was about $300 \mu \mathrm{m}$. The double track resolution was $7 \mathrm{~mm}$ at $90 \%$ confidence level [55].

\section{Trigger and data acquisition}

\subsection{Introduction}

After each beam crossing, the trigger decides whether an $\mathrm{e}^{+} \mathrm{e}^{-}$interaction took place and if so, whether the event should be recorded. This function is performed at three levels of increasing complexity, reducing the 45$\mathrm{kHz}$ beam crossing rate to a few $\mathrm{Hz}$ of tape writing rate. The quality of the accepted data is monitored. The detector parameters are also monitored, for detector calibration and for safety (slow control).

The online computer system consists of a VAX 8800 , clustered with five smaller VAX monitoring each of the main detector components and the trigger system. The cluster is connected via Ethernet to VME crates and personal computers which acquire the slow control data and to VAX stations for event display and run control. The data acquisition uses mostly FASTBUS, chosen for its speed and flexibility. The system includes ample buffering capacity to allow asynchronous operation without contributing to the dead time.

\subsection{Trigger ievels}

All the detectors are read by the front end electronics for each beam crossing. In addition to the main data, each detector provides coarser and less accurate trigger data available in digitized form within a few $\mu \mathrm{s}$. The level 1 trigger analyzes the trigger data and either initiates the digitization of the main data or clears the front end electronics before the next beam crossing, i.e. within $22 \mu \mathrm{s}$, so that negative decisions at level 1 do not contribute to the dead time. After a positive decision, the detector data are digitized and stored within $500 \mu \mathrm{s}$ in multievent buffers and the system is readied for the next beam crossing. The expected level 1 trigger rate is $100 \mathrm{~Hz}$, corresponding to a dead time of $5 \%$, but the system can cope with a trigger rate up to $500 \mathrm{~Hz}$ with a $25 \%$ dead time.

After a level 1 trigger, the trigger data are further analyzed by four programmable processors (XOP), each one disposing of an average time per event of $8 \mathrm{~ms}$ under the most adverse condition of a $500-\mathrm{Hz}$ trigger rate. This level 2 trigger should reduce the rate of accepted events by a factor of 10 .

The level 3 trigger is performed by three 3081/E which have at their disposal the original trigger data, the results of the calculations performed by the level 1 and level 2 processors and the complete sei of digitized data fium all the detector components. These data are then used for a further filtering of the events. The time available for each event is now about 10 times longer and the information much more complex, thus a further reduction of a factor 10 on the number of accepted events is expected from this third level trigger. Only when this last scrutiny arrives at a positive decision, the event is transmitted to the main data acquisition computer to be written on tape at an expected rate of $1 \mathrm{~Hz}$ at the $Z^{0}$ peak.

\subsection{Data flow}

The digitized data for each detector component may be stored in several event memories situated in the "detector" FASTBUS crates (fig. 116). Data from all parts of the detector are then collected together and sent to the central crate. The merging of the data from separate substreams is performed by the combination of three FASTBUS modules, a general purpose master (GPM) [56], a block mover (BM) [57] and a dual slave memory (DSM) [58]. The GPM is a FASTBUS master which monitors the status of the source memories (LRS1892 and DSM) and sets up the block transfer parameters in the BM. The BM then takes up mastership of the crate and cable FASTBUS segments and performs a series of block moving from several source memories to one destination memory (DSM). The function of the BM is controlled by a microcode, and a 


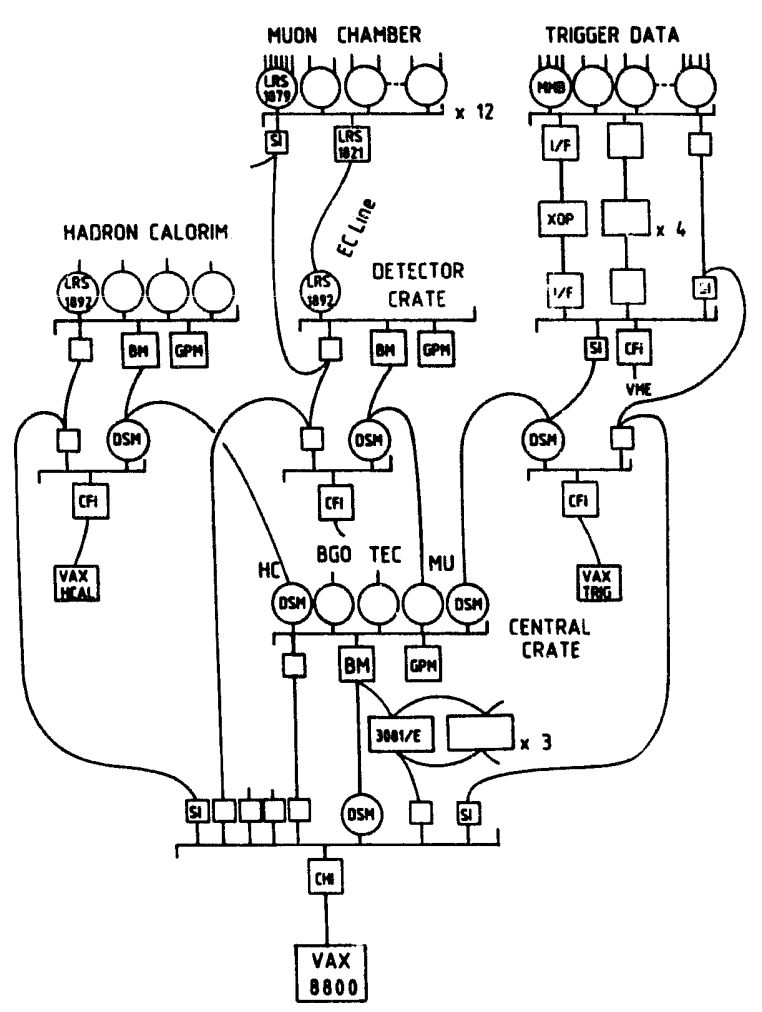

Fig. 116. The $L 3$ data acquisition system.

variety of FASTBUS operations can be easily implemented. Furthermore, each detector component has, interfaced to its computer, a dedicated FASTBUS crate with a DSM which is on the same cable segment as the DSM of the central crate. The data from each detector component can therefore also be monitored in "spy mode" on the detector compu:er.

An important exception to this procedure is the TEC. Data reduction processors (DRP) are used at the front end to reduce the large amount of data generated by this detector by a factor of 20 . Since the DRP need 5 ms to process a typical event and transfer it to the buffer, they are only started by the positive decision of the level 2 trigger.

One of the DSM on the central crate receives from the level 2 processors the trigger data together with the level 2 decision. The GPM examines this decision. If it is negative, the event is simply erased from each subdetector DSM and from the front end memories of the TEC. If it is positive, the DRP of the TEC are started and the $\mathrm{BM}$ is instructed to merge the information coming from the different subdetectors and to send them to one of the three $3081 /$ E computing units which are on its cable segment.

The central crate is connected to the VAX 8800 with the 4-MB/s CERN host interface (CHI). The CHI can perform any FASTBUS action, check and monitor collected data and transfer them to the VAX.

\subsection{The level I trigger}

The level 1 trigger is simply the logical OR of trigger conditions from different sources: calorimetric trigger, muon trigger, TEC trigger and scintillator trigger.

\subsubsection{Calorimetric trigger}

The calorimetric trigger processes the information given by the electromagnetic and hadronic calorimeters and by the luminosity monitor (256, 384 and 32 channels respectively); it reaches a final decision $16.8 \mu \mathrm{s}$ after the beam crossing and is built with 350 CAMAC modules forming a digitizer part and a processor part. The CAMAC bus is only used for initial loading and for testing purposes. The trigger data flow on front panel differential ECL buses, 16 bits wide. Data from different buses can merge together making synchronous operation of all the modules necessary. This is achieved with a centralized and programmable timing source which distributes the strobes to the whole trigger system. CAMAC 16 channels fast encoding and readout ADC (FERA) modules, LRS4300, are used for a fast digitization of the information whereas the processor part is built around memory lookup units (MLU LRS2372) and arithmetic logic units (ALU LRS2378).

For the electromagnetic calorimeter, the outputs of 30 BGO crystals are grouped together to obiain a segmentation of 32 in $\Phi$ and of 8 in $\Theta$. These 256 signals are digitized in $8.5 \mu \mathrm{s}$ with an 11-bit range and a sensitivity of $25 \mathrm{MeV} /$ bit. The luminosity monitors give 16 signals on either side, corresponding to 16 segments in $\Phi$. Two signals are extracted from each hadron calorimeter module. The first (layer $\mathbf{A}$ ) corresponds to the chambers in the first interaction length. The remaining chambers form the layer B. The last modules on each side of the barrel do not have a layer $A$ since they can only be reached by particles from the interaction region which have crossed the $A$ layer of other modules (fig. 117). The resulting segmentation is 16 in $\Phi$ and 11 (layer A) or 13 (layer B) in $\Theta$, with a total of 384 channels. These signals are digitized in $4.8 \mu \mathrm{s}$ with a 10-bit range and $50 \mathrm{MeV} / \mathrm{bit}$.

$$
\text { CALOPHETRIC FRIGCER SIGHAL SEGMENTATION IN RO VIEW }
$$

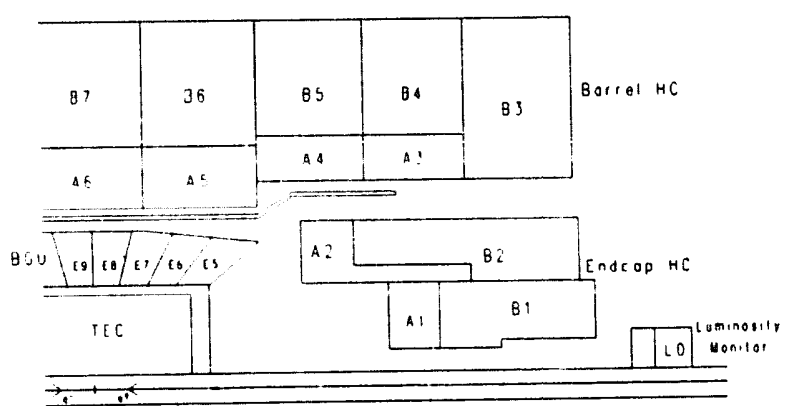

Fig. 117. Detector segmentation for trigger signals. 
The data digitized by the FERA are read at 120 $\mathrm{ns} /$ word via the front panel bus; the data from two groups of FERA are multiplexed into a single bus and go at $60 \mathrm{~ns} /$ word through memory lookup units (MLU, LRS2372) which equalize the energy scale for all the calorimeter parts and remove the coherent noise contribution. The output of these MLU requires only 12 bits, and one of the highest bits is programmed to give a signal ("hit") if the energy recorded in the single trigger cell is above a given threshold, different for each calorimeter and function of $\theta$. These signals are used by the "hit counting trigger" as described below. The energy data from the MLU are presented on a bus to the second part of the processor at one word every 60 $n s$. The operations of sum and accumulate, addition and subtraction are performed by fast arithmetic and logic units (ALU, LRS2378) [59]; more complex operations are performed by MLU; intermediate data are stored in data stacks (DS, LRS2375). The patterns of timing signals necessary to drive the system are stored in fast memories (ECLine Drivers, or ED) [60]. Extensive use is also made of the bus switch (BSW) [61] module which latches two 16-bit ECL words and multiplexes data from two buses onto a single bus. In order to speed up the data flow, data from the hadron calorimeter are presented in parallel on seven different buses. Since the BGO data need a longer time to be digitized, they are ready when the flow of the $\mathrm{HC}$ data is terminated and they are sent on the last four of the same buses.

For each calorimeter (BGO, HC layer A. HC layer B), the data corresponding to a fixed $\Theta$ and different $\Phi$ values are summed together ( $\Phi$-sums) and similarly the data corresponding to a fixed $\Phi$ and different values of $\Theta(\Theta$-sums). The 640 trigger data are therefore reduced to 96 elements, which are used to perform the following trigger calculations:

Total energy trigger: The $\Phi$ sums are added over $\Theta$ to give the total electromagnetic, total hadronic and total energies. A partial sum is also performed limited to the central part of the detector. A trigger is given if the total energy is above a predefined threshold, which can be different if the energy is localized in the central part of the detector and can also depend on the ratio of electromagnetic to hadronic energy.

Cluster trigger: A cluster is a localized deposit of energy observed in different detector layers at the same $\Theta, \Phi$ coordinates, the energy threshold depending on it e calorimeters involved. A trigger is given if at least one cluster is found. Clusters are searched for independently in the two projections $R-\Theta$ and $R-\Phi$. The information from TEC and the scintillation courters can contribute to the definition of a cluster in the $R-\Phi$ view.

Single photon trigger: A cluster in the BGO is accepted even if its energy is very low, provided it accounts almost for the totality of the e.m. eneryy detected. No track must be detected by the TEC in coincidence with the cluster in the $R-\Phi$ projection.

Hit counting trigger: A trigger is given if the number of hits detected by the MLU in the first part of the processor is above a certain threshold.

In addition, the luminosity monitor data are presented on one bus and the data corresponcing to the two opposite sections of the detector are stored in two data stacks. A search is made for two high energy hits with strong back to back correlation which is an indication of Bhabha events and gives rise to a luminosity trigger. If a high energy hit is measured in only one monitor and, in addition, some energy is measured in the central part of the detector, the event is accepted as a single tag trigger.

\subsubsection{Muon trigger}

The trigger processor searches for tracks originating at the interaction point. It is composed of 120 CAMAC modules, all controlled by a single source of synchronous clock pulses. The main module is the hit array (HA, LRS2376), a $1 \mathrm{~kb}$ memory with two modes of operation, load and search, corresponding to the two steps of the track finding procedure defined below.

Each muon chamber wire sends a signal to the 96 channel FASTBUS TDC. The same signals are also sent via the FASTBUS module auxiliary connector to a personality card (PC) for the trigger purpose. Inputs from two adjacent wires are ORed and stored in a hit memory. The 48 hit memories are enabled for the duration of the chambers drift time $(1.2 \mu \mathrm{s})$. For the trigger the chamber wires are grouped into "trigger cells" which, for the $P$ chambers, coincide with the physical chamber cells (16 to 24 wires) while, for the $Z$ chambers, the information from the adjacent two wires of each double plane are combined together to give a single trigger cell. The personality card controller (PCC) is a FASTBUS module which accesses the PC and decides about the presence of a track in each trigger cell. For the $P$ chambers the PCC groups the input data according to the trigger cell segmentation, counts the number of fired hit memories within the trigger cell and compares it with a preset threshold number. If the number of hits is above threshold, the PCC encodes a 10-bit word with the layer number ( 2 bits), the cell number ( 7 bits) and one control bit. For the $Z$ chamber the processing is simpler since a trigger cell corresponds to a single hit memory. The data are processed by the PCC in parallel for all octants and therefore a total of 16 buses, 8 for the $P$ and 8 for the $Z$ cinambers, feed the trigger data into the trigger processor at the same time.

In the load step, the hit addresses are presented by the PCC on each bus to a number of HA ( 4 for the $Z$ chambers and 3 for the $P$ chambers). Oniy one HA, whose layer number assignment agrees with the layer number presented on the bus, picks up the cell number, 
interprets it as a load address and writes 1 in the corresponding location of its hit memory.

For the search step a number of possible "roads" of tracks originating from the interaction point are predefined. Each road is parameterized by its central cell number and its half width in each one of the three chambers layers. Each road corresponds to a certain region of the muon production angle in $\Phi$, the transverse momentum and the electric charge. All the possible tracks with $P_{1}>1 \mathrm{GeV} / c$ are defined. Track finding starts after all the PCC have finished loading the trigger data. A control stack presents road parameters of each layer to the respective HA and interrogates whether the HA finds any hits within the specified range. In the $R-\Phi$ plane the three layers of $P$ chambers are used. The answers from the three HA are collected at one place and the track is identified if a coincidence condition (3-fold or majority of 2) is satisfied between the three layers. The large bending of the low momentum muons can cause the road pattern to interest two neighboring octants. For these roads the answers of the HA corresponding to the two octants are collected together. In the $R-Z$ plane ( $Z$ chambers) there are four chamber layers and a similar procedure is followed. The following conditions give a trigger:

Single muon trigger: A muon track is defined in the $\boldsymbol{R}-\boldsymbol{\Phi}$ plane as a coincidence of all three chamber layers and in the $R-Z$ plane as a coincidence of all four chamber layers. At least one octant should have a track identified both in the $R-\Phi$ and $R-Z$ planes. This trigger is effective in the region $44^{\circ}<\Theta<136^{\circ}$ where the chambers have a complete $\Theta$ coverage.

Di-muon trigger: A muon track is defined by a twofold coincidence of any two layers both in the $R-\Phi$ and $R-Z$ planes but at least two octants should have a track identified and the two tracks should satisfy a coplanarity condition. This trigger is useful for the larger angular region $36^{\circ}<\Theta<144^{\circ}$ where the angular coverage of the chambers is incomplete.

Small-angle muon trigger: A muon track is defined by the presence in the $R-\Phi$ plane of a single hit in the inner $P$ chambers and in the $R-Z$ plane by a coincidence of both layers of inner $Z$ chambers. Furthermore, if a track is found in the forward half of the detector, another should be found in the backward half. There is also the possibility to accept two tracks both in the forward or backward half of the detector. This trigger covers the small angle region $\left(36^{\circ}<\Theta<44^{\circ}, 136^{\circ}<\right.$ $\Theta<144^{\circ}$ ) where oniy one layer of $P$ chambers is available.

\subsubsection{TEC trigger}

For each of the 24 outer TEC segments the trigger uses 14 wires out of 54 [62]. The data from all TEC segments are processed in parallel: a segment divider module (SD) divides the total drift time into two bins and solves the left-right ambiguities by means of signals induced on the grids located at the right and left of the anode plane [63]. In this manner, the $R-\Phi$ plane is subdivided into $96 \Phi$ bins. Thus, after processing by the SD modules, the level 1 TEC trigger information consists of a $96 \times 14$ bit matrix.

For each $\Phi$ bin, a track finder module (TF) searches for the presence of tracks which leave the TEC in this $\Phi$ bin and originate at the beam line. The 96 sets of 14 signals are used to address random access memories (RAM) which contain the topologies of all relevant tracks, allowing for the effects of inefficiencies and additional hits. The TF perform the track search in parallel in less than $1 \mu \mathrm{s}$. Three types of tracks are defined:

- Tracks with polar angle $\Theta>42^{\circ}$ and transverse momentum $P_{1}>600 \mathrm{MeV} / c$ are contained in a single $\Phi$ bin and the track search can be realized with a 16-kbit RAM addressed by the 14 data bits from this single $\Phi$ bin. The lower limit of $42^{\circ}$ on $\Theta$ is set by the TEC geometry: tracks with smaller polar angles miss the outermost wires.

- Tracks with $\Theta>42^{\circ}$ and $P_{\mathrm{t}}>150 \mathrm{MeV} / c$ cross up to three adjacent $\Phi$ bins. The corresponding SD modules provide therefore $5 \times 14$ bits of information. i.e. one time 14 bits from the "reference bin" plus two times $2 \times 14$ bits from the two pairs of bins adjacent to the central one. To keep the size of RAM memory to a manageable level, it is necessary to reduce this information. The 14 bits of each $\Phi$ bin are grouped radially two by two. The grouping is an AND or OR, independently programmable for each pair of bits. If the background conditions are good, all pairs can be set to OR to maximize trigger efficiency. If background conditions are bad, all pairs can be set to AND so as to increase the rejection of spurious tracks. As a compromise, if the background is localized at small radii, the signals in this region can be set to $A N D$, the others at larger radii to OR.

- Forward tracks with $\Theta>25^{\circ}$ and $P_{\mathrm{t}}>100$ $\mathrm{MeV} / c$. The search for small polar angle tracks is performed with the seven innermost wires. The algorithm described above is applied, but no AND or OR is now necessary. Since the same masks are used, the $P_{t}$ cut is lower for the forward tracks.

Becalise of the difference in maximum drift time between the inncr and outer wires, the search for the forward iracks is performed first. in $5 \mu \mathrm{s}$ from the beam crossing time. After a further $5 \mu s$ the search for the central tracks can begin. The search is completed within $11 \mu \mathrm{s}$. At the end of the search a track adder module counts the total number of tracks found, the number of clusters (a cluster being defined as any number of contiguous tracks) and the number of pairs of tracks with an acoplanarity angle smaller than some programmable value. The level 1 TEC trigger decision is taken 
on the basis of these numbers, and the information on the tracks found is sent to the calorimetric trigger processor as well as to the level 2 trigger.

\subsubsection{Scintillator trigger}

The scintillator triggers, based on the signals of 30 barrel and 32 end cap counters, are the following:

Multiplicity trigger: It requires a coincidence of two out of the $\mathbf{3 0}$ barrel counters and is used to trigger on cosmic muons in calibration runs. During LEP operation this trigger is efficier:' for events with two muons or two hadronic jets, thus it serves as a simple backup trigger for the li-muon and cluster triggers, and is useful to monitor their efficiency.

Coincidence pattern: This allows us to select predefined patterns of hits, chosen from the barrel and the end cap counters, and in coincidence with the beam gate. The following trigger conditions can be programmed:

- two counters in different barrel octants;

- one barrel counter and one end cap counter;

- one forward and one backward end cap counter;

- two forward (or two backward) end cap counters in different quadrants.

The scintillator signals are also sent to the calorimetric trigger to contribute to the decision of the cluster trigger.

\subsubsection{Trigger control}

The trigger control implementis the final level 1 trigger decision and synchronizes the data acquisition and the level 1 trigger with the beam crossing signal. Before every beam crossing, the trigger control checks if all the detector components are ready for a new event. If they are, it sends a CLEAR signal to reset the whole DAQ system, increments the event number counter and starts the next cycle; then the beam crossing signal is sent out to sample the data of the whole detector and to initiate the level 1 trigger processors. The results from the trigger processors are returned and evaluated before the next beam crossing. If the event is rejected, the system is reset by the CLEAR signal and ready for the next event. If the event is accepted, an ACCEPT signal is sent to the subdetectors to start the data conversion and buffering. During this time the subdetectors must set FALSE on their READY line to prevent further events from being accepted

The results of the level 1 trigger processors are combined into a 16-bit trigger word. Together with a 16-bit event number it is sent to the subdetectors and attached to the evert data. This provides a check of the data integrity at the event building stage. The trigger word also provides information for event selection of the online monitoring and is a guide for the level 2 and level 3 computations. Trigger control also provides timing signals for taking cosmic data or synchronizing test signal generators to calibrate the detector and test the DAQ system.

\subsection{The level 2 trigger}

The level 2 trigger scheme is shown in fig. 116. The multiport multievent buffer (MMB) is an 8 events deep, 256 words per event, first in/first out input memory [64]. After each beam crossing, all data (4 k 16-bits words) delivered by the front end trigger digitizers are stored in parallel in the $60 \mathrm{ECL}$ input ports at the speed of $60 \mathrm{~ns}$ per word. The final and some intermediate results of all the level 1 trigger processors are also stored. One of the ports receives the trigger word and the event number generated by the trigger control logic. The levei 2 trigger receives also information which was not available in time to be processed by the level 1 . In particular, it receives charge and drift time information from the charge division wires of the TEC, which allow to define the track coordinates in three dimensions [62].

Data acquisition management is hard wired and synchronized by signals delivered by the level 1 trigger. All the data banks connected to the input ports are overwritten at each beam crossing until a level 1 signal validates the event. This increments the input event pointer and the status register.

Four XOP processors work in round robin mode between two FASTBUS segments. XOP is a fast trigger processor designed at CERN [65] and fully integrated in FASTBUS [66]. It is microprogrammable and executes 192 bits wide microinstructions in $100 \mathrm{~ns}$ on 16-bits data words. It communicates with the external world via a VMF interface ıor program loading and a FASTBUS interface [67] for data taking and processing. When idle, each XOP competes for mastership of the input crate segment. The winner reads the MMB status word. If positive, it reads the earliest event in each memory and decrements each MMB status register, thus building a complete event with its event number. This readout is decoupled from level 1 operation since the MMB memory provides a simultaneous random read/write access. When the readout is completed the XOP releases mastership and starts computation.

The main purpose of this computation is to reduce the event rate by:

a) the detection of the clustered energy in the BGO and two lateral layers of hadron calorimeter by correlating them in a full $\Theta-\Phi$ plane;

b) the longitudinal and transverse energy balance of the clustered energy;

c) the recognition of the vertex along the beam axis by using the charge division wire of the TEC chamber. The programmability of the XOP provides ample flexibility in the selection criteria.

After the computation is finished, the XOP arbitrates for mastership on the output segment in order to 
write data and results, includirg the final decision on the acceptance of the event, into the DSM on the central crate. The same data can be read in spy mode by the DSM connected to the trigger computer and used to monitor the trigger.

\subsection{The level 3 irigger}

The level 3 trigger, made by three 3081/E emulators, is embedded in the main flow of the data acquisition. The $3081 / \mathrm{E}$ is a computer developed at SLAC and CERN which emulates a subset of the IBM 370 instruction set and runs as fast as an IBM 370/168. Each $3081 / E$ is equipped with a FASTBUS interface with two cable segment ports completely symmetric and independent, one used for data input, another for data output: 1/O operations can be done by one port at a time, while the control and status register (CSR\#0) is common to both ports and can be simultaneously accessed by them to know the status of the machine. Through this interface the 3081/E becomes a FASTBUS slave geographically addressable on cable segment. As a consequence the level 3 architecture relies on the use of intercommunicating masters on both input and output segments to control all the ispects of the data flow. When a $3081 / E$ is free its input responds to $T$-pin broadcast and receives a complete event at a speed of $16 \mathrm{MB} / \mathrm{s}$. It then starts a computation for the event selection.

Unlike the level 1 and level 2 triggers, for which only' the trigger data with coarse granularity and lower resolution are available for the trigger decision. the level 3 trigger has access to the complete digitized data with finer granularity and higher resolution. A complicated algorithm written in FORTRAN and tested with the offline computer is executed. The selection of good events is based on

a) the correlation of the energy deposited in the BGO and hadron calorimeters,

b) the reconstruction of muon track in the $Z$ chambers, c) the reconstruction of the vertex in the TEC chamber. A steering program minimizes the processing time by properly arranging the sequence of the above calculations.

In case of a positive level 3 decision, a service request (SR) signal is asserted on the output port and the FASTBUS computer interface (CHI) transfers the data from the emulator memory to the main data acquistion computer fôr tape writing.

\subsection{The online computers and sofincure}

The online computer system of the L3 apparatus is sketched in fig. 118. It is centered on a cluster of DEC VAX. Three TA78 tape drives, two TA90 cartridge drives and $10 \mathrm{~GB}$ of disks are connected via the $\mathrm{I} / \mathrm{O}$

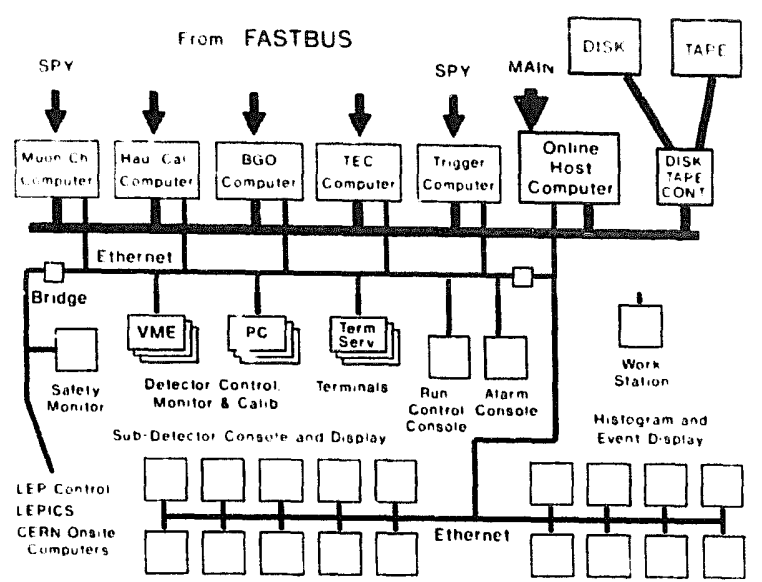

Fig. 118. The L3 online computer system.

server HSC70. The main data acquisition computer is a VAX 8800 . Five smaller VAX devoted to each of the main detector components and one to monitor the trigger system are attached to the cluster. These VAX also form, via Ethernet, a local area VAX cluster (LAVC) with a number of VAXstations used as intelligent command consoles and as multiwindow graphics displays. One of the VAXstations is used for the run control. The slow control data are gathered by a series of VME crates and PC, which are linked to the cluster via a second Ethernet.

The on!ine software has been developed using the MODEL. library package developed at CERN. Starting. stopping, etc., of both the event data flow and monitoring processes is under operator direction via a master process running on the rur control VAXstation. When the run is active the event data arrive from the $3081 / \mathrm{E}$ into the CHI. A process running on the 8800 transfers the event out of the $\mathrm{CHI}$ and into a buffer in the 8809 memory. The process then assigns an event number and finishes the formatting. A subsequent process picks up the event from memory and writes it to the tape in machine independent format. Concurrently with the event writing, but at a lower priority, monitoring processes are reading events out of the memory buffer on a sampling basis. On the 8800 , only those monitoring tasks which require data from more than one detector component are performed, including the partial reconstruction of events using the L3 offline software. The outputs of these tasks are buffered on disk for event display and for further analysis. A bank of eight VAXstations is available for this.

The data for the monitoring and control of individual detector components are acquired by the detector computers which can access the detector component data on a sampling hasis, possibly subject to predefined conditicns. While the 8800 receives only the events accepted by the level 3 trigger, the individual detecior computeis can have access to all the events accepted by 
the level 1 trigger, ind also during calibration and setup periods. Each one of these computers has a console VAXstation running prosesses for analysis and display of the data.

The L3 detector is monitored for overall safety and detector integrity. The LEP General Safety Services microVAX continuously monitors conditions in the experimental area to detect and prevent hazards such as the accumulation of explosive gases. This microVAX is linked to the LEP control room via Ethernet, and, should a dangerous situation begin to develop and not be responded to, the power to the area is cut. A function of the slow control microVAX is to respond to these promptings and alert operators to initiate less drastic responses. These two microVAX are on an uninterruptible power source. Other functions of the programs running on the slow control VAX are to ensure that the data taking conditions are within specified tolerances and alert the operators should they change. Should the operators not respond, it can inform devices controlling other parts of the detector operation. All these $m^{\circ}$ sages are logged for later investigation.

For precise reconstruction of the events it is also necessary to set, monitor and record many detector parameters at a much finer level than that needed for slow control and safety purposes. This task is distributed (fig. 118) between the PC and VME microprocessors which control the hardware and the VAX computers. Periodically, or on request, th - microprocessors transfer the current settings to disk files on the VAX cluster. As a debugging tool. these files can be immediately dumped to consoles. Periodically, processes on the 8800 pick up this data and format it into ZEBRA banks and insert it into the event data stream. Irom which it is written to tape. An alternate path is to send these hanks ur: an Ethernet link to the L3 offline computer LEPICS, where they are processed for inclusion in the master database. The online copy of the database is also updated by these processes. This database is used for the event reconstruction and display mentioned above. Filling it during data taking ensures that reconstruction results acquired online will be reproducible offline. Another function of the online database is to allow monitoring of these parameters over time. The online database has a validity interval reaching back about one week which allows these parameters and their correlations to be histogrammed versus time.

\section{LEP3NET: L3's intercontinental computer network}

\section{I. The origin and evolution of LEP3NET}

L3 recognized in 1981 that completion of the detector and preparation of the data analysis would require a sophisticated system of computer links between the members of the Collaboration [68,69]. Prior to data taking, L3 physicists, enginee:s and tuchnicians in the US, Europe and Asia would need a means of instant electronic communication with their colleagues at CERN and at the collaborating institutions. Because of the precision and diversity of the L 3 detection systems and the richness of the L.3 physics program, the offline software developinent task is significantly larger than for any previous high energy physics experiment, requiring several hundred man years of work. It was therefore vital to get the means to allow physicists to work efficiently while at their home sites and to coordinate with and contribute to the mainstream of software development work going on at CERN. This led to LEP3NET: L3's intercontinental computer network.

LEP3NET began in 1982-1983 as a pilot project, involving links between Caltech, DESY and CERN, for the MARK $J$ and L3 experiments. The network was initially based on the public packet switching networks TELENET (US), DATEX-P (Federal Republic of Germany) and TELEPAC (Switzerland). Public network usage soon expanded to include links to Michigan, Amsterdam, Madrid and Aachen. Dedicated links to Annecy, Lyon, Rome and Naples continued to be used, with progressive migration (wholly or in part) to $\mathrm{X} 25$.

The performance of international links using the public packet networks was a fraction of the maximum line speed (typically 4.8 or $9.6 \mathrm{~kb} / \mathrm{s}$ in 1983) and the charges for the volume of data sent were quite high. The very fact that charges were volume-sensitive led to the unacceptable situation that monthly bills could rise to unpredictable levels. These factors soon made leased lines the preferred solution. Following a feasibility demonstration using a leaseci satellite link between Caltech and Princeton in 1985-1986, the network became operational on January 30,1986 when a transatlantic line was delivered by AT \& $\mathrm{T}$ and the Swiss PTT. From then on, the network lines and switches demonstrated mean time between failures measured in months and mean times to repair in hours in most cases.

The current topology of LEP3NET is shown in fig. 119. LF.P3NET is widely used by high energy physicists in the USA and in Europe. Increases in network bandwidth have been achieved through more sophisticated and expensive modems on the existing LEP3NET lines. In July 1986. Codex modems running at $16.8 \mathrm{~kb} / \mathrm{s}$ were installed on the LEP3NET transatlantic lines. The Caltech-MIT and MIr-LBL links are now running at $19.2 \mathrm{~kb} / \mathrm{s}$. The CAMTEC packet switches at CERN and MIT were upgraded to models capable of switching packets between lines running at up to $64 \mathrm{~kb} / \mathrm{s}$. Most recently, the presence of the higher speed X25 TELEFILE switches at MIT and CERN, which are key sites both on LEP3NET and ESNET, has led to the decision to move some key lines to the faster switches. In September 1988. the speed of the LEP3NET transatlantic 


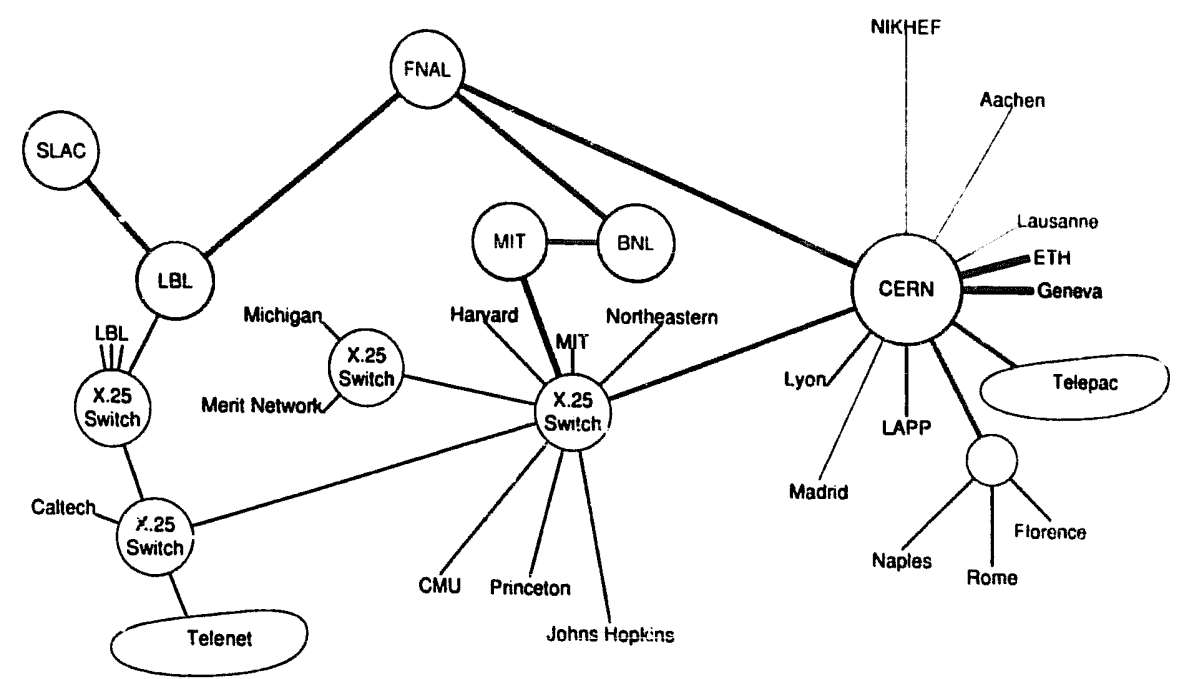

Fig. 119. The LEP3NET and ESNET-X.25.

line was increased to $56 \mathrm{~kb} / \mathrm{s}$. This became economically feasible with the advent of the TAT-8 Transatlantic optical fiber cable.

\subsection{LEP3NET design elements}

The design of LEP3NET takes into account the wurldwide spread of the Collaboration, the limited manpower available to construct, develop and maintain the network and above all the limited budget. Some of the design elements are:

1) The data communications protocols are based on the internationally recognized standard X25, which sends the data from source to destination in packets. including information for error checking, and error recovery procedures to ensure that the data is received error-free. The X25 protocol was chosen for LEP3NET for a number of compelling reasons. $\mathrm{X} 25$ is the onl: way to reach ma ny of L3's European collaborators and it allows transparcnt connections tc ${ }^{-}$established across combined leased-line and publ: networks. The X25 protocols are supported, and off-the-shelf interfaces are provided, by all majo: computer manufacturers.

2) In order to support the full range of services needed by the physicists, several sets of higher-level protocols run on top of X25. This includes DECNET and the Coloured Books protocois. The Coloured Books protocol has been implemented on many machines to provide a high level user interface on top of the $\times 25$ protocol and has particularly valuable features for retries and resumption after interruptions.

3) The data communications equipment is all standard, commercially available. This includes the packet switches and computer interfaces. The CAMTEC packet switches were chosen on the basis of performance/cost, programming flexibility and proven long term reliability.

\subsection{LEP3NET network services}

The facilities offered by LEP3NET are detailed below.

\subsubsection{Terminal access to remote computers}

Any LEP3NET computer can make terminal calls directly to any cther L.EP3NET hos:. The X25 protocol avoids the overheads of routing through intermediate hosts and terrestrial lines minimizing propagation delays. Except under heavy load, the switching delays are still small compared with the cable transit times for coast-to-coast or transatlantic calls. Remote use of fullscreen facilities (such as the VAX TPU editor or IBM VM/CMS) over 11000 miles of LEP3NET cable is relatively comfortable, even though the 200 -ms delay for remotely echoed characters is quite visible. Witn the connection of L3's "LEPICS" IBM 3090 to LEP3NET in February 1988, it became evident that full screen access to LEPICS, using character by character remote echoing, often places a heavy load on LE: 3NET. Even with an unloaded network, propagation delays rinake this mode of access slow. A software package supporting IBM " $327 x$ " full screen terminal emulation on VAX computers has been obtained. The package maintairis a loral screen image on the VAX at the user's site and only changes are transmitted to and from the IBM whenever the "enter" or "attention" key is pressed. The package also provides extensive support for "key mapping", which is needed to make remote use convenient on non-IBM remote terminals by emulation of the PF (permanent function) keys, whose use is an integral part of working on an IBM VM/CMS compuier system. 


\subsubsection{Electronic mail}

Electronic mail over LEP3NET has removed almost all need for paper mail and the directness and speed of mail exchange has also decreased the need for long listance phone calls. In many cases, the exchange of many mail messages within a short time takes the place of a telephone discussion, with the added advantage of giving time to think and to formulate ideas clearly. The electronic mail over LEP3NET has the advantages of immediacy (in most cases) and :eliability. Mail can be sent to computers which are down for maintenance or disconnected due to temporary line problems; delivery of the mail will take place as soon as the connection is reestablished.

\subsubsection{File transfer}

The Culoured Books file transfer system supports transfer of programs and binary files between many types of computers (e.g. VAX, IBM, Gould, etc). The system offers data compression, automatic re-tries, and automatic resurnption from "marks" after any interruption. File transfer requests are sent to a manager process and control is immediately returned to the user while the transfer is handled by a background sub-process. These features make it easy to transfer programs and small data samples of up to a few tens of megabytes. Even though the line bandwidth makes such transfers last many hours, they can be confidently leift to run while other work continues.

\subsubsection{Decnet}

DECNET "data link mapping" circuits have been established over LEP3NET between Caltech and CERN and between I"arvard and CERN. These circuits link the European and USA HEP DECNET in a way that is transparent to most users. In spite of the obvious value of a link between USA and European HEP, and the great range of facilities offered by DECNET, L3 itself continues to use the Coloured Books network software for most network traffic. The Coloured Books software's greater immunity to network disturbances, its more efficient use of the limited bandwidth, and its availability on non-DEC machines, make it the natural choice for most serious work. Nevertheless, LEP3NET does not impose a choice of network software. Coloured Books and DECNET can coexist on the same machines using the same physical connections.

\subsection{Examples of LEP3NET usage}

Even before the official start of LEP3NET, the Princeton group, which is responsible for the BGO electronics and readout. was making heavy use of a LEP3NET pilot project linking them to CERN via a Princeton-Caltech satellite link and the Telenet gateway at Caltech. The Princeton group has continued their heavy network usage which has permitted them to continue to contribute to the analysis of BGO test beam data. Network access to the test beam data acquisition system has proved invaluable for the preparation and debugging of the system by experts who were usually not at CERN.

The muon chamber reconstruction software has been written almost exclusively by people resident in the USA, but used the latest version of the SIGEL3 L3 Monte Carlo simulation program developed at CERN. Soon after the startup of LEP3NET, the complete software base needed to run the Monte Carlo (some $\mathbf{5 0}$ MB) was transferred to MIT and regularly updated. More recently, with the creation of a CERN-based branch of the muon chamber software group, there has been a frequent interchange of new software.

Coordination of the $\mathrm{L} 3$ computing and software tusks requires an active involvement in many areas. Network access is also used for examining and resolving problems with the network. In addition, specific projects at participating institutions require access to software developed at other institutes in the collaboration. For example, the Monte Carlo study of light collection in crystals under calibration by the RFQ system reyuired the collection of software from L3's European collaborators.

\subsection{Future network needs and LEP3NET}

The success of LEP3NET in offering a high quality, general purpose service at a cost comparable to that of the line rentals has made it an attractive model for the fiture of HEP networking in the US. The HEPNET Technical Coordinating Conımittee incorporated much of LEP3NET's experience and desiyn principles in its recommendations for the ESNET X25 backbone.

In the near future, the main problem of LEP3NET will be its limited speed. Early analyses of the L3 network needs made in 1984 and $1985[68,69]$ showed that the principal links to CERN should reach speeds in the range of $224 \mathrm{~kb} / \mathrm{s}$ soon after LEP startup, rising above the $\mathrm{Mb} / \mathrm{s}$ range when LEP Phase I reaches its design luminosity. These high speeds are primarily needed to support remote interactive terminal sessions, rapid transmission of a minute fraction of the data, file transfer for program development and physics analysis, along with several other services. Each physics group is expected to need network access to CERN in the 64 $\mathrm{kb} / \mathrm{s}$ range or higher.

This early analysis has since been confirmed by detailed analyses performed by the HEPNET Review Committee (HRC) for high energy physics as a whole [70]. This has led the HRC to recommend provision of $\mathrm{Mb} / \mathrm{s}$ speeds on the ESNET backbones used by HEP by 1989. NSFNET also has planned links to Europe in this speed range, aithough these links will be shared by 
a. large, broadly based scientific community. In Europe. several initiatives which seek to provide $2-\mathrm{Mb} / \mathrm{s}$ links to CERN have been launched, in response to the findings of study groups on the computing and networking needs for the LEP experiments [71,72].

Although it is hoped that some of L3's future high bandwidth networking needs will be satisfied by the expansion of "general purpose" networks, such as ESNET or NSFNET in the US, LEP3NET will continue to be L3's primary network for the next few years. Only LEP3NET will be able to provide the full range of higher level protocols, rapid interactive access, and guaranteed bandwidth for L3's highest priority needs.

\section{References}

[1] L3 collaboration, Letter of Intent (January 1982); L3 collaboration, Technical Proposal (May 1983).

[2] D. Lehm, G. Petrucci and G. Stefanini, technical note ALEPH 87-55, note 87-10 (August 1987).

[3] U. Becker et al., Nucl. Instr. and Meth. 180 (1981) 61; P. Duinker et al., Nucl. Instr. and Meth. 201 (1982) 351.

[4] P. Duinker et al., Nucl. Instr. and Meth. A273 (1988) 814.

[5] U. Becker et al., Nucl. Instr. and Meth. 128 (1975) 593; C. Willmott, Nucl. Instr. and Meth. A263 (1988) 10.

[6] W.E. Toth, Prototype octant construction and evaluation with production phase recommendations, CSDL-R-188j (1987) unpublished.

[7] N.K. Chnabra and K. Narender, Computerized structure analysis of the muon octant frame, CSDL-R-2011 (1987) unpublished.

[8] M.G. Dix et al., NASA Technical Briefs (1981) 319.

[9] P.G. Seiler et al., The Laser Beacon, L3 Muon Chamber Group Internal Note, 85-4 (1985) unpublished.

[10] H. Anderhub, M. Devereux and P.G. Seiler. Nucl. Instr. and Meth. 176 (1980) 323.

[11] J.C. Guv, F. Hartjes and J. Konijn, Nucl. Instr. and Meth. 204 (1982) 77 :

F. Hartjes and J. Konijn, Nucl. Instr. and Meth. 217 (1983) 311;

F. Hartjes, J. Konijn and Y. Peng, Nucl. Instr. and Meth. A269 (1988) 544.

[12] P. Rewiersma, NIKHEF-H, Internal note, L3 wire amplifier (1986) unpublished, hybrid made by Philips.

[13] P. Rewiersma, NIKHEF-H, Internal note DISC.DOC27.03.87PR, unpublished.

[14] S. Burov et al., CERN-EP/88-84.

[15] A. Arefiev et al., Nucl. Instr. and Meth. A275 (1989) 71.

[16] A Bobchenko et al., Sov. J. Nucl. 30 (1979) 805;

S. Denisov et al., Nucl. Phys. B61 (1973) 62;

J. Allaby e! a!., Sov. I. Nucl. Phys. 13 (1971) 295:

Yu. Gorin et al., Sov. J. Nucl. Phys. 18 (1974) 173.

[17) Yu. Galaktionov et al., Nucl. Instr. and Meth. A251 (1986) 258;

A. Arefiev et al., Nucl. Instr. and Meth. 285 (1989) 403.

[18] B. Bleichert et al., Nucl. Instr. and Meth. A254 (1987) 529.

[19] U. Martyn and J.F. Zhou, Nucl. Instr. and Meth. A256 (1987) 143.
[20] R. D'Alessandro, preprint DFF $\$ 72$ (1988):

E. Gallo, preprint DFF \# 76 (1988).

[21] M. Bocciolini et al., Nucl. Instr. and Meth. A257 (1987) 509.

[22] M. Bocciolini et al., Nucl. Instr. and Meth. A257 (1988) 548.

[23] R. Sumner, Nucl. Instr. and Meth. A265 (1988) 252.

[24] For BGO machining, see:

- M. Lebeau et J.C. Le Marec. Usinage des cristaux de BGO, LAPP, Dossier ANVAR 2101 (1985);

- M. Lebeau et H. Vey, Rodage et polissage des cristaux de BGO, LAPP, Rapport interne 19/02/85:

- M. Lebeau, Recherche fondamentale et transfert technologique: une collaboration LAPP (Annecy)-SIC (Shanghai), Rev. Technique APAVE 236 (1986) 67.

[25] M. Schneegans, Nucl. Instr. and Meth. A257 (1987) 528. and. Test and preparation of BGO crystals for the L3 calorimeter, to be submitted to Nucl. Instr. and Meth.

[26] C. Laviron and P. Lecoq, CERN report L3-416, (1986).

[27] M. Lebeau, LAPP report L3-58.4 (1986).

[28] M. Chemarin, IPN Lyon, CERN-L3-394 (1985); M. Chemarin and M. El Kacimi, CERN-L3/88-621 (1988).

[29] EUCLID: a computer aided design software from MATRA-DATAVISION-France.

[30] Technical specification LAPP (April 5, 1985); Selected company: Elicotteri Meridionali, Centro Compositi Sud, Gruppo Agusta Frosinone, Italy: CERN contract LB/CR/987 (June 6, 1987).

[31] M. Lebeau. J. Mater. Sci. Lett. 4 (1985) 779.

[32] Finite Element Calculation code MODULEF developed by INRIA-France.

[33] Diodes for the L3 BGO Calorimeter: Quality tests and mouning. PITHA $88 / 21$.

[34] M. Goyot, B. Ille. P. Lebrun, J.P. Martin. Nucl. Instr. and Meth. A263 (1988) 180.

[35] M. Bosteels. L.EP/IM/MB/Y N (1985).

[36] Roll-bound Aluminum inflated cooling panels hot-rolled by ALCAN SPA. Milano, Iualy.

[37] J.A. Slakken et al.. CERN-EP/89-16: P.E. Kaaret. thesis, Princeton University (1989), ref. DoE/ER/367;,'ju.

[38] J.A. Bakken et al., Nucl. Instr. and Meth. A275 (1989) 81.

[39] B. Borgia et al., Internal Report Dept. di Fisica, Università di Roma "La Sapienza" 925 (1988) and Nucl. Instr. and M:th. A278 (1989) 699.

[40] J.A. Bakken et al., Nucl. Instr. and Meth. A270 (1988) 397.

[41] C. Rippich, "Monte Carlo Results for the L3 Luminosity Monitor", (1984) and L3 Technical Proposal (1983) chap. 9.

[42] C. Rippich, CMU-HEP 86-14 (1986) (this reference deals with an earlier version of the monitor design).

[43] G. Von Holtey, CERN yellow report 88-06. vol. 2. p. 896 .

[44] J.F. Crawford et a1. Nucl. Inctr. and Meth. 127 (1975) 173.

[45] F.A. Berends and R. Kleiss, Nucl. Phys. B228 (1983) 537.

146] H. Anderhub et al. Nucl. Instr. and Meth. A252 (1986) 357.

[47] H. Anderhub et al., Nucl. Instr. and Meth. A263 (1988) 1. 
[48] H. Anderhub et al., Nucl. Instr. and Meth. A265 (1988) 50.

[49] A. Böhm et al., Nucl. Instr. and Meth. A273 (1988) 471.

[50] U. Röser et al., A shaping amplifier for high resolution drift chambers operated at low drift velocity with Flash ADC readout, in preparation.

[51] H. Akbari et al., Multianode readout of scintillating fibers for L3 Vertex Chamber calibration, to be published in Proc. Scintillating Fiber Workshop at Fermilab, 1988.

[52] H. Akbari et al., Performance of the multianode readout of scintillating fibers used for calibration of the $L 3$ vertex chamb to in preparation.

[53] G. Viertel, Research and Development on Time Expansion Chambers, Invited Talk given at the Int. Symp. on Position Sensitive Detectors in High Energy Physics, Dubna, USSR, 1987.

[54] K. Deiters, The $Z$ detector of the $L 3$ experiment, ibid.

[55] W. Friebel et al., PHE 87-08, Zeuthen (1987).

[56] H. Muller and R. Vachon CERN EP, Electronics Note 85-02.

[07] L. Pregernig, The Block Mover, presented at the 1985 JEEE Nuclear Science Symposium, San Francisco, CA, USA.

[58] H. Maller, C.M. Story and S. Falciano, CERN-EP Electronics Note 87-04.

[59] F. Cesaroni, S. Di Marco, E. Gennari and S. Gentile Nucl. Instr. and Meth. A260 (1987) 425.

[60] LAPP/SCAIME, Ecline Driver User's Manual, Technical report.
[61] F. Cesaroni, E. Gennari, S. Gentile and P. Pacchiarotti, Nucl. Instr. and Meth. A260 (1987) 546.

[62] M. Bourquin et al., Charged particle trigger for the L3 experiment, Proc. Int. Conf. on the Impact of Digital Microelectronics and Microprocessors on Particle Physics, ICTP, Trieste, 1988.

[63] A. Boehm et al., Nucl. Instr. and Meth. A273 (1988) 4712.

[64] J. Lecoq, M. Moynot and G. Perrot, F-682B Multi-port Multi-event Buffer (MMB), LAPP Internal Report 23.01.85 revised 10.04.87.

[65] T. Lingjaerde, Proc. Topical Conf. on the Application of Microprocessors to High Energy Physics Experiments (1981) CERN, Geneva, CERN report 81-07.

[66] J. Lecoq, M. Moynot, G. Perrot, P. Baehler and C. Ljuslin, Proc. Fastbus Software Workshop (1985) CERN, Geneva, CERN Report 85-15.

[67] G. Perrot, XFMI/XFSI Specifications, LAPP Technical Document, revised 1987.

[68] H. Newman, Caltech DoE and NSF Research Proposal CALT-68-1109 (1984).

[69] R. Mount, L3 Technical Note No. 351 (1985).

[70] High Energy Physics Computer Networking, Report of the HEPNET Review Committee (1988).

[71] D. Williams (ed), The Computing Needs of the LEP Experiments (the MUSCLE Report), CERN DD and EP Divisions (1988).

[72] D. Williams (ed.), Green Book II Study: -omputing for Experiments, CERN, (1988). 